\title{
Eddy-Kovarianz Messungen über einem tropischen Regenwald in komplexem Gelände
}

\author{
Dissertation \\ zur Erlangung des Doktorgrades \\ der Mathematisch-Naturwissenschaftlichen Fakultäten \\ der Georg-August-Universität zu Göttingen
}

vorgelegt von

Thomas Roß

aus Bad Oldesloe

Göttingen, 2007 
D7

Referent: Prof. Dr. Andreas Tilgner

Korreferent: Prof. Dr. Gode Gravenhorst

Tag der mündlichen Prüfung: 20.06.07 


\section{Inhaltsverzeichnis}

1 Einleitung 1

2 Messungen und Methoden 5

2.1 Messungen turbulenter Flüsse . . . . . . . . . . . . . 5

2.1.1 Theoretische Grundlagen und Technik der Eddy-Kovarianz

Methode . . . . . . . . . . . 6

2.1.2 Die Stoffbilanzgleichung: Ableitung der relevanten Terme 6

2.1.2.1 Vereinfachung der Stoffbilanzgleichung nach

Lee $(1998) \ldots \ldots$. . . . . . . . . . . . 8

2.1.2.2 Ergänzung der Stoffbilanzgleichung nach Au-

binet $(2003)$. . . . . . . . . . . . . . . 10

2.1.3 Beschreibung des Messgeländes . . . . . . . . . 11

2.1.4 Messturm und Instrumentierung . . . . . . . . . . . . 13

2.1.4.1 Das Ultraschallanemometer (USA-1, METEK) 15

2.1.4.2 Der "Open-Path" Infrarotgasanalysator (IR-

GA 7500, LICOR) . . . . . . . . . . . 15

2.1.4.2.1 Querempfindlichkeit ....... 17

2.1.5 Korrekturen . . . . . . . . . . . . . . 17

2.1.5.1 Webb, Pearman und Leuning (WPL) Term . 17

2.1.5.2 Sensibler Wärmestrom nach Schontanus . . . 19

2.1.6 Das Planar-Fit Verfahren . . . . . . . . . . . . 20

2.1.7 Beurteilung der Datenqualität . . . . . . . . . . . . 22

2.1.7.1 Der Stationaritätstest . . . . . . . . . . . 22

2.1.7.2 Integrale Turbulenzcharakteristik . . . . . . . 23

2.1.8 Prozedur der Datennachbearbeitung . . . . . . . . 23

2.2 Verfahren zur Datenlückenschließung . . . . . . . . . . . . 24

2.2.1 Modellierung der assimilatorischen $\mathrm{CO}_{2}$-Flüsse . . . . . 26

2.2.1.1 Lichtsättigungsfunktion nach Michaelis-Menten 26

2.2.2 Untersuchung der saisonalen Variabilität der respiratorischen $\mathrm{CO}_{2}$-Flüsse . . . . . . . . . . . . . . . 26 
2.2.2.1 Das $u_{*}$-Filterkriterium . . . . . . . . . 27

2.2.3 Schließung von Datenlücken (Gap-Filling) . . . . . . . 29

2.3 Ähnlichkeitstheorie . . . . . . . . . . . . . . . . . . 31

2.3.1 Das Schließungsproblem . . . . . . . . . . 31

2.3.2 Monin-Obukhov Ähnlichkeitstheorie . . . . . . . . . . 32

2.4 3-D Grenzschichtmodellierung . . . . . . . . . . . . . . . . . 33

2.4.1 Theorie des $E$ - $\omega$ Modells . . . . . . . . . . . . . . . 33

2.4.2 Strahlungsregime und Vegetation . . . . . . . . . 38

2.4.3 Relief und Koordinatensystem . . . . . . . . . . . 38

2.5 Footprintanalyse . . . . . . . . . . . . . . . . . . . 39

2.5.1 Prinzip des NSG-Modells nach Sogachev et al. . . . . . 40

2.5.2 Das analytische Modell nach Kormann-Meixner . . . . 41

2.5.3 Zusammenfassung . . . . . . . . . . . . . 43

3 Ergebnisse $\quad 45$

3.1 Meteorologie und Turbulenz am Standort . . . . . . . . . . . . 45

3.1.1 Das lokale Windsystem . . . . . . . . . . . . . 45

3.1.2 Meteorologische Eigenschaften . . . . . . . . . . . . 48

3.2 Bowen-Verhältnis . . . . . . . . . . . . . . . . . . . . . . . . . 49

3.3 Methoden zur Sicherung der Datenqualität . . . . . . . . . 50

3.3.1 Der Turmeffekt: Beeinträchtigung der Datenqualität durch den Turm . . . . . . . . . . . . 50

3.3 .2 Die Footprintanalyse . . . . . . . . . . . . . 52

3.3.3 Untersuchung schichthöhenkonstanter Flüsse . . . . . 56

3.3.4 Test zur integralen Turbulenzcharakteristik . . . . . . . 57

3.3.5 Stationaritätstest und $u_{*}$-Filterkriterium . . . . . . 59

3.3.6 Energiebilanzschließung . . . . . . . . . . . . . 62

3.3.7 Die Planar-Fit Methode . . . . . . . . . . . . . . . 62

3.3.8 Zusammenfassung . . . . . . . . . . . . . 67

3.4 Berücksichtigung advektiver Strömungen . . . . . . . . . . 68

3.4.1 Abschätzung advektiver Einflüsse bei Nacht (katabatische Schwereflüsse) . . . . . . . . . . . . 68

3.4.2 Berechnung advektiver $\mathrm{CO}_{2}$-Flüsse mittels eines $E$ - $\omega$

Modells für neutrale Schichtung . . . . . . . . . . 76

3.4.2.1 Modellierung des vertikalen $\mathrm{CO}_{2}$-Flusses unter besonderer Berücksichtigung der Quellen und Senken . . . . . . . . . . . . . 77

3.4.2.2 Modellierung der vertikalen $\mathrm{CO}_{2}$-Flüsse in Abhängigkeit von der Windrichtung . . . . . 80

3.5 Untersuchung der Kohlenstoffbilanz . . . . . . . . . . . . 85

3.5.1 Aufteilung des $\mathrm{CO}_{2}$-Flusses in seine Komponenten . . . 85 
3.5.1.1 Darstellung der Nettoassimilation mit dem Modell von Michaelis-Menten . . . . . . . 86

3.5.1.2 Saisonalität der respiratorischen $\mathrm{CO}_{2}$-Flüsse . 92

3.5.2 Berechnung des Nettoökosystemaustausches . . . . . . 94

3.5.2.1 Kumulative Darstellung des Nettoökosystemaustausches und der Komponentenflüsse . . 95

3.5.2.2 Koordinatensystemvergleich . . . . . . . . 95

3.5.2.3 Resultate aus den Berechnungen zur vertikalen Advektion . . . . . . . . . . . . . . . . . . . 98

4 Zusammenfassung und Diskussion

A Notation

A.1 Symbolverzeichnis für das E-w-Modell . . . . . . . . . . . 108

A.2 Allgemeines Symbolverzeichnis . . . . . . . . . . . . . . . 109 


\section{Kapitel 1}

\section{Einleitung}

Nach dem aktuellen Sachstandsbericht 2007 des International Panel for Climate Change (IPCC) lässt sich kaum mehr bezweifeln: Die globale Erwärmung stellt die größte Herausforderung des 20. Jahrhunderts dar. Nach dem Urteil des IPCC sind die derzeitig beobachteten Temperaturerhöhungen mit über 90\%-iger Sicherheit auf anthropogen bedingte Treibhausgaszuwächse zurückzuführen. Etwa $60 \%$ der beobachteten globalen Erwärmung ist auf den Anstieg der $\mathrm{CO}_{2}$ Konzentration von ca. $280 \mu \mathrm{mol} / \mathrm{mol}$ in der vorindustriellen Phase auf $380 \mu \mathrm{mol} / \mathrm{mol}$ heute zurückzuführen [Gra04]. In Zahlen ausgedrückt hat sich die Erde binnen der letzten 100 Jahre im Mittel um $0.74^{\circ} \mathrm{C}$ erwärmt.

Zur Bestimmung des zukünftigen Klimas mithilfe globaler Zirkulationsmodelle ist das Verständnis über die Rückkopplungsprozesse im globalen Kohlenstoffkreislauf von besonderer Bedeutung [SMSD86]. In diesem Sinne stellen Sensibilitätsuntersuchungen der $\mathrm{CO}_{2}$-Flüsse eines gegebenen Ökosystems in bezug auf die verschiedenen Umweltvariablen eine wichtige Voraussetzung für Modellierung, Scaling-up Verfahren und Voraussagen dar [WMN98].

Aber auch die Frage, ob alle globalen Ökosysteme zusammen eine NettoQuelle oder Netto-Senke darstellen, wird bereits seit 30 Jahren kontrovers diskutiert. Woodwell forderte 1978 [WWR78], dass die Ökosysteme insgesamt eine Quelle darstellen sollten. Dagegen wies Broeker [BTSP79] auf die Diskrepanz zwischen den bekannten $\mathrm{CO}_{2}$ Quellen und den niedrigeren Raten, mit denen $\mathrm{CO}_{2}$ in der Atmosphäre auftrat, hin. Er prägte den Begriff von der verlorenen Senke (missing sink). Nach einfacher Abschätzung werden ca. $7-8 G t(C) y r^{-1}$ durch Industrie und Waldrodung emittiert, etwa $3 G t(C)$ verbleiben in der Atmosphäre und von den restlichen $4-5 G t(C)$ werden $2 G t(C)$ von den Ozeanen aufgenommen. Also bleiben $2-3 G t(C)$ übrig, 
von denen angenommen wird, dass sie von den terrestrischen Ökosystemen aufgenommen werden. Die Frage ist wo? An dieser Stelle tritt die sogenannte Eddy-Kovarianz (EK) Methode als die wichtigste Methode zur Bestimmung des $\mathrm{CO}_{2}$-Austauschs auf Ökosystemebene in den Vordergrund.

Seit Anfang 1990 stehen Infrarotgasanlysatoren mit ausreichend hoher Frequenz zur Verfügung, sodass turbulente $\mathrm{CO}_{2}$ - und $\mathrm{H}_{2} \mathrm{O}$-Flüsse in geeigneter Weise gemessen werden können. So ist es möglich geworden, festzustellen, welche Ökosysteme wo auf der Erde Quellen oder Senken für $\mathrm{CO}_{2}$ darstellen. Der resultierende Fluss, welcher vom Quellgebiet ausgehend vom Sensor detektiert wird, wird als Nettoökosystemaustausch bezeichnet. Auch lassen sich mittels der EK-Methode Abhängigkeiten zwischen $\mathrm{CO}_{2}$-Fluss und den verschiedenen Umweltvariablen modellieren. Informationen, ohne welche Klimamodellierungen, Upscaling-Verfahren und Prognosen nicht möglich wären [LGM95, WMN98].

Geschah die Beprobung anfangs vor allem im Rahmen von kurzen Kampagnen und vorwiegend über landwirtschaftlichen Flächen unter nahezu idealen Voraussetzungen (homogenes Terrain), werden heutzutage in der Regel Langzeitmessungen zur Bestimmung des Nettoökosystemaustauschs zwischen den verschiedensten Vegetationsformen und Atmosphäre durchgeführt [ $\left.\mathrm{TAB}^{+} 03\right]$. An hunderten von Standorten weltweit werden heutzutage kontinuierlich EKMessungen betrieben.

Verständlicherweise existieren umso weniger Informationen über Ökosysteme, je abgelegener sie sind. Besonders wenig Messungen wurden bis heute über tropischen Wäldern durchgeführt. Dies gilt insbesondere für den südostasiatischen Raum. Daher stellt die vorliegende Arbeit, die auf ersten Langzeitmessungen aus der Region Bariri auf der Insel Sulawesi in Indonesien beruht, einen weiteren wichtigen Mosaikstein zum Verständnis globaler Klimaprozesse dar.

Doch es gibt auch Einschränkungen, welche mit der Anwendung der EKMethode verbunden sind. Die Unterlage sollte möglichst eben und die Vegetation möglichst homogen verteilt sein. Andernfalls lässt sich die Methode nicht ohne weiteres auf eine Ein-Punkt Messung reduzieren. Auf der anderen Seite sind ca. 28\% der Wälder der Erde Hochlandwälder (Mountain Forests). Insbesondere in den Tropen nimmt der Anteil von Tieflandwäldern aufgrund der durch den Menschen verursachten Landnutzungsänderungen (slash and burn) ständig ab. In der Praxis versucht man deswegen häufig einen Kompromiss zwischen ausreichend homogenen Geländebedingungen einerseits und dem Wunsch ein bestimmtes Ökosystem zu beproben andererseits, zu finden.

Unter diesen Voraussetzungen ist bei Anwendung der EK-Methode darauf zu achten, dass weitere Messungen zur Validierung hinzugezogen werden. Als 
wichtigste zusätzliche Messung zur Korrektur des vertikalen $\mathrm{CO}_{2}$-Austauschs ist die Speichertermmessung zu nennen $\left[\mathrm{AGI}^{+} 00\right]$. Außerdem setzt sich in jüngster Zeit immer mehr durch, vertikale sowie horizontale Advektionstermmessungen durchzuführen [FBV03, $\left.\mathrm{MCM}^{+} 05\right]$. Insbesondere in der Nacht und bei stabiler atmosphärischer Schichtung, so zeigen zahlreiche Untersuchungen, tritt häufig ein nächtliches $\mathrm{CO}_{2}$-Flussdefizit auf, das nicht allein auf Probleme mit der EK-Methode zurückzuführen ist [ML02, GMF ${ }^{+}$96], sondern vielmehr auf einen bisher unbeachteten advektiven Transport von $\mathrm{CO}_{2}$ hinweist $\left[\mathrm{ABC}^{+}\right.$05, Lee98, LH02, ML02, $\left.\mathrm{TAB}^{+} 03, \mathrm{FBO}^{+} 01\right]$.

Vor diesem Hintergrund besteht das wesentliche Ziel dieser Arbeit darin, unsere Messungen über dem alten tropischen Hochregenwaldbestand bestmöglich zu überprüfen, um schließlich zu belastbaren jährlichen Raten des Nettoökosystemaustauschs zu gelangen. Die zugrundeliegende Idee dieser Arbeit basiert auf dem Ansatz, aus experimentellen Untersuchungen auf der einen Seite und nummerische 3d-Modellrechnungen auf der anderen Seite größtmöglichen wechselseitigen Nutzen für die Beurteilung des Standorts zu ziehen. 


\section{Kapitel 2}

\section{Messungen und Methoden}

\subsection{Messungen turbulenter Flüsse}

Als atmosphärische Grenzschicht wird jener Teil der Troposphäre bezeichnet, in welcher die Effekte der Oberfläche (Reibung, Erwärmung und Abkühlung) in Zeitskalen geringer als ein Tag fallen und in welcher signifikante Flüsse von Impuls, Wärme und anderen Skalaren durch turbulente Bewegungen stattfindet, deren Größenordnung von der Höhe der Grenzschicht oder darunter gelegen ist [Gar99]. Sie wird nach oben durch eine statisch stabile Luftschicht mit intermittierender Turbulenz begrenzt. Es handelt sich um eine Zwischenschicht (entrainment-layer), über die ein Austausch von Luftmassen mit der darüberliegenden Troposphäre stattfindet. Der Aufbau der atmosphärischen Grenzschicht wird wesentlich und unmittelbar durch die Wechselwirkung zwischen der Erdoberfläche und der Atmosphäre bestimmt (turbulenter Austausch von Impuls, Wärme und Wasserdampf, bedingt durch Strahlungsprozesse und den Einfluss der Bodenreibung). Diese im Mittel ca. 1-2 km hohe Schicht unterliegt einem sehr variablen Tagesgang. Nach Sonnenaufgang sorgen turbulente Wärmeströme für eine rasche Erwärmung der Atmosphäre. So entsteht nach Auflösen der nächtlichen Inversion eine Schicht mit guter Durchmischung (mixing layer). Bereits vor Sonnenuntergang findet die sogenannte Bodeninversion statt. In der Nachfolge bildet sich eine stabile Grenzschicht von nur geringer Mächtigkeit [Stu84].

Die unteren von der Winddrehung unbeeinflussten $10 \%$ der atmosphärischen Grenzschicht werden als Boden- oder Prandtl1-Schicht bezeichnet. Die

\footnotetext{
${ }^{1}$ Prandtl, Ludwig (1875 - 1953), Göttinger Physiker und Entwickler der Grenzschichttheorie
} 
Prandtl-Schicht ist zur Bestimmung von $\mathrm{CO}_{2}$ - und anderen Stoffbilanzen von besonderem Interesse, da $\mathrm{CO}_{2^{-}}, \mathrm{H}_{2} \mathrm{O}$ - oder Energieflüsse in diesem Bereich höhenkonstant gemessen werden können. Aus diesem speziellen Grunde wurden weiter unten Untersuchungen vorgenommenen, die den Zweck haben die gewählte Höhe, in der über dem Regenwald gemessen wurde, zu validieren.

\subsubsection{Theoretische Grundlagen und Technik der Eddy- Kovarianz Methode}

Mikrometeorologische Flussmessungen nach der EK-Methode waren lange Zeit lediglich Gegenstand der Forschung. Die aktuellen Fragen zum Klimawandel haben jedoch im Verlauf der letzten 10 Jahre dazu geführt, dass turbulente $\mathrm{CO}_{2}$-Flussmessungen heute beinahe selbstverständlich zur Bestimmung des Nettoökosystemaustauschs (NEE) durchgeführt werden. Dabei beruht die EK-Methode auf einer Reihe von Vereinfachungen und Annahmen. Insbesondere durch die notwendige Ausweitung der Messstandorte in immer entlegenere und komplexere Regionen, ergibt sich die Notwendigkeit, wichtige einschränkende Maßnahmen, wie horizontale Homogenität der Unterlage und stationäre Austauschbedingungen, noch gründlicher zu untersuchen als das bisher geschah. Start und Ausgangspunkt zum Verständnis der strömungsmechanisch relevanten Terme ist die Stoffbilanzgleichung.

\subsubsection{Die Stoffbilanzgleichung: Ableitung der relevan- ten Terme}

Die Stoffbilanzgleichung dient zur Beschreibung von Wärmetransportvorgängen als auch für den Transport von Gasen und Beimengungen:

$$
\frac{\partial C}{\partial t}+u_{i} \frac{\partial(C)}{\partial x_{i}}=\nu_{c} \frac{\partial^{2}}{\partial x_{i}^{2}} C+S_{c} .
$$

Der erste Term von links gibt die Speicheränderung des betrachteten Skalars an und wird kurz als Speicherterm bezeichnet. Term (2) beschreibt die räumliche Änderung ${ }^{2}$ des totalen Flusses, Term (3) mit $\nu_{c}$, dem molekularen Diffusionskoeffizienten, den diffusiven Transport und $S_{c}$ die Quell- oder Senkenstärke im betrachteten infinitesimalen Volumen. Durch Zerlegung ${ }^{3}$ der

\footnotetext{
${ }^{2}$ Hier und im Folgenden wird die Einstein'sche Summenkonvention benutzt. Über identische Indizes innerhalb eines Terms wird von eins bis drei summiert.

${ }^{3}$ Die sogenannte Reynolds'sche Zerlegung (nach Osborne Reynolds, engl. Physiker) einer turbulenten Größe: $x=\bar{x}+x^{\prime}$.
} 
betrachteten Größen in einen zeitlich gemittelten und einen fluktuierenden Anteil und anschließender Mittelung ergibt sich aus Gleichg. 2.1:

$$
\frac{\partial \bar{C}}{\partial t}+\bar{u}_{i} \frac{\partial}{\partial x_{i}} \bar{C}+\frac{\partial \overline{u_{i}^{\prime} C^{\prime}}}{\partial x_{i}}=\nu_{c} \frac{\partial^{2}}{\partial x_{i}^{2}} \bar{C}+S_{c}
$$

Hier fand, um die turbulente Advektion in "Flussform" zu bringen, die Kontinuitätsgleichung $\overline{u_{i}^{\prime}} \partial C^{\prime} / \partial x_{i}=\partial \overline{u_{i}^{\prime} C^{\prime}} / \partial x_{i}$ Anwendung. Durch Integration und Vernachlässigung des molekularen Diffusionsterms (die molekulare Diffusion ist 4-6 Größenordnungen kleiner, als der Beitrag der anderen Terme) ergibt sich die absolute Stoffbilanz in einem Kontrollvolumen (vergl. Abb. 2.1):

$$
\iiint_{V} S=\iiint_{V}\left(\frac{\partial \bar{C}}{\partial t}+\bar{u}_{i} \frac{\partial}{\partial x_{i}} \bar{C}+\frac{\partial \overline{u_{i}^{\prime} C^{\prime}}}{\partial x_{i}}\right) .
$$

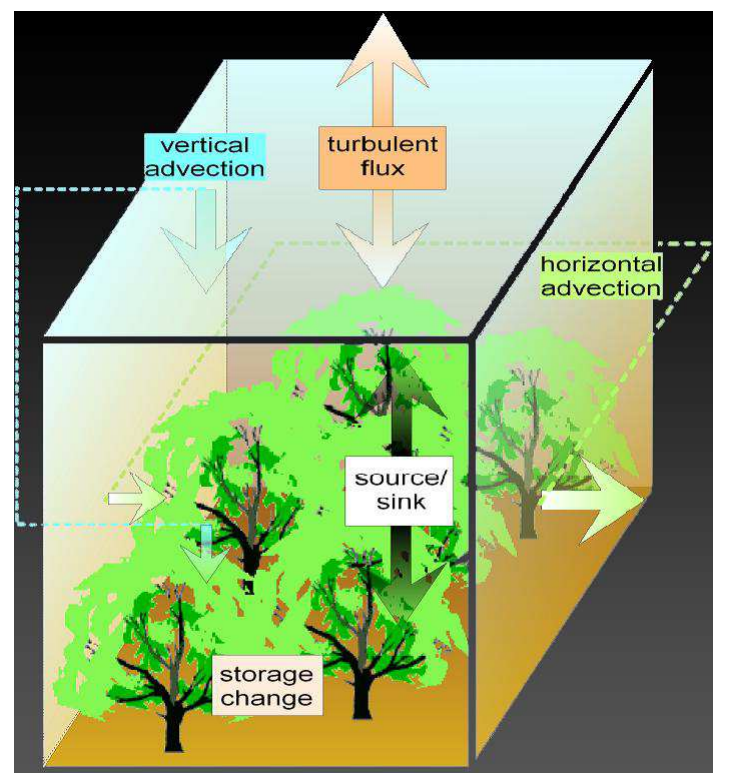

Abbildung 2.1: Kartesisches Kontrollvolumen über einem Waldbestand (Quelle (nicht publ.): C. Feigenwinter). 


\subsubsection{Vereinfachung der Stoffbilanzgleichung nach Lee (1998)}

Im Verlauf der neunziger Jahren - mit der Bildung regionaler Netzwerke (EuroFlux ${ }^{4}$, AmeriFlux $^{5}$, OzFlux $^{6}$ und AsiaFlux ${ }^{7}$ ) - fanden verschiedene Gruppen, dass mit der EK-Methode bei ungünstigen Standort- und Atmosphärenbedingungen nächtliche $\mathrm{CO}_{2}$-Flüsse systematisch unterschätzt werden $\left[\mathrm{GMF}^{+}\right.$96, LAM98, $\mathrm{BHN}^{+}$96]. In der Folge publizierte Lee [Lee98] einen einfachen Lösungsansatz, den er mit den verhandenen Schwierigkeiten hinsichtlich der Energiebilanzschließung über hoher Vegetation motiviert. Er nennt advektive Prozesse, die er auf mesoskalige Luftmassenbewegungen verbunden mit stationären konvektiven Zellen, nächtliche Kaltluftabflüsse (drainage flows oder katabatic winds), inhomogenes Anströmgebiet und einige weitere Punkte zurückführt.

Als Ziel seiner Arbeit gibt er vor, möglicherweise auftretende horizontale Strömungsdivergenz oder -konvergenz zu untersuchen. Vereinfachend beschränkt sich Lee bei seiner Betrachtung auf zwei Dimensionen ( $x$ entlang der lokalen Hauptwindrichtung und $z$ vertikal dazu). Schließlich trifft er, ausgehend von einem gedachten Kontrollvolumen $\{2 L$ (Länge $) \times 1$ (Einheitsbreite $) \times$ $z_{r}$ (Messhöhe)\} [Fin99], folgende Annahmen:

1. Die Stoffbilanzgleichung kann als eindimensional betrachtet werden, auch wenn das zugrundeliegende Strömungsfeld ein Zweidimensionales ist. Die Werte der Variablen in $(x=0, z)$ entsprechen ihrem horizontalen Mittel:

$$
\frac{1}{2 L} \int_{-L}^{+L} \phi(x, z) d x \simeq \phi(0, z),
$$

mit $\phi$, den betrachteten Variablen, und $2 L$, der Länge des Kontrollvolumens in $x$-Richtung

2. Überall innerhalb der Strömung gilt:

$$
\bar{u} \frac{\partial \bar{C}}{\partial x} \ll \bar{w} \frac{\partial \bar{C}}{\partial z} .
$$

3. Ebenfalls überall innerhalb der Strömung gilt:

$$
\frac{\partial \overline{u^{\prime} C^{\prime}}}{\partial x} \ll \frac{\partial \overline{w^{\prime} C^{\prime}}}{\partial z} .
$$

\footnotetext{
${ }^{4}$ http://www.unitus.it/dipartimenti/disafri/progetti/eflux/euro.html

${ }^{5}$ http://public.ornl.gov/ameriflux/

${ }^{6} \mathrm{http}: / /$ www.dar.csiro.au/lai/ozflux/index.html

${ }^{7} \mathrm{http}: / /$ www-cger2.nies.go.jp/asiaflux/index.html
} 

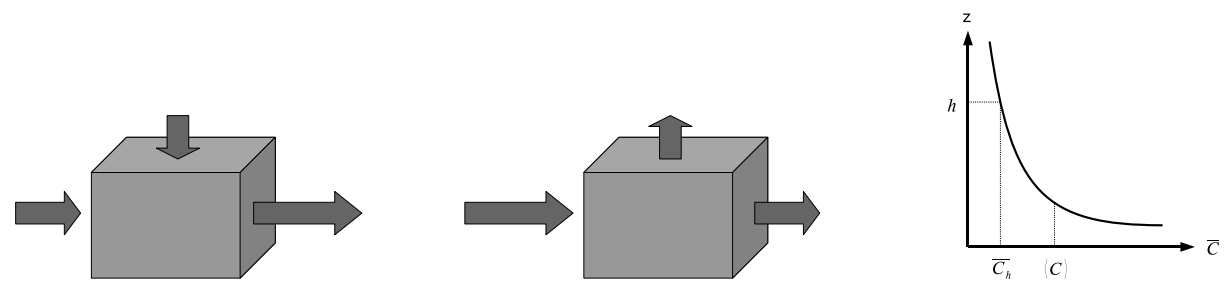

Abbildung 2.2: Schematische Darstellung einer konvergenten (links) bzw. divergenten Strömung (rechts) durch ein Kontrollvolumen. Außerdem dargestellt (zur Illustration von $\langle\bar{C}\rangle$ ): Ein vertikales $\mathrm{CO}_{2}$-Konzentrationsprofil, wie es typischerweise in der Nacht auftritt.

4. Mit $\bar{w}_{r}$, der mittleren vertikalen Geschwindigkeit und $z_{r}$, der Messhöhe, gilt:

$$
\frac{\partial \bar{w}}{\partial z} \simeq \frac{w_{r}}{z_{r}}
$$

Unter Berücksichtigung der Annahmen (1) bis (4) ist Gleichg. 2.3 über das Kontrollvolumen zu integrieren. Nach anschließender nochmaliger partieller Integration von $\bar{w} \partial \bar{C} / \partial z$ von Null bis $z_{r}$ folgt schließlich

$$
N E E=\int_{0}^{z_{r}} \frac{\partial \bar{C}}{\partial t} d z+\left(\overline{w^{\prime} C^{\prime}}\right)_{r}+\bar{w}_{r}\left(\bar{C}_{r}-\langle\bar{C}\rangle\right),
$$

wobei

$$
\langle\bar{C}\rangle=\frac{1}{z_{r}} \int_{0}^{z_{r}} \bar{C} d z
$$

Abbildung 2.2 veranschaulicht die betrachteten Situationen schematisch. Der publizierte Ansatz in [Lee98] gab aufgrund der vielen Simplifizierungen Anlass zu viel Diskussion, weshalb kurz darauf ein schriftlicher Kommentar von Finnigan [Fin99] erschien. Die wesentlichen Kritikpunkte an den von Lee getroffenen Annahmen seien hier kurz wiedergegeben:

- Zu Punkt 1: Finnigan führt verschiedene Mechanismen an, die im Fall einer zweidimensionalen Strömung dazu führen, dass es zu Variationen im mittleren Windfeld kommt. Als besonders wichtig nennt er den Einfluss unterschiedlicher Strahlungsflussstärken über komplexem Gelände, Rauhigkeitsinhomogenitäten, Änderungen der turbulenten Schubspannungen in ihrer Wechselwirkung mit der 2d-Strömung [KF94] und großräumig konvektive Prozesse [RWCH92]. Auch in dem Fall konstanter Oberflächenflüsse führten die letzten drei Punkte zu horizontalen Variationen bei turbulenten Flüssen. 
- Der Annahme 2 spricht Finnigan die allgemeine Berechtigung ab, indem er auf ein einfaches numerisches Strömungsexperiment hinweist, welches die Vernachlässigung der horizontalen gegenüber der vertikalen Advektion nur in einem Ausnahmefall rechtfertigt. Anhand von Modellrechnungen werden zweidimensionale Zirkulationsmuster untersucht, die allein in der Nähe sogenannter stagnierender Stromlinien dominierenden advektiven Transport in vertikaler Richtung zeigen. Die tatsächliche Relation von vertikaler zu horizontaler Advektion hinge letztlich von den unter Punkt 1 genannten Faktoren ab.

- Die dritte Annahme ist laut Finnigan schwer zu widerlegen. Die turbulenten Flussdivergenzterme sind auch im zweidimensionalen Fall das Resultat komplexer Wechselwirkungen mit dem turbulenten Strömungsfeld. Die Erkenntnisse aus dem Modell von Raupach [RWCH92] scheinen die Annahme jedoch zu rechtfertigen, falls die Messhöhe gering ist im Vergleich zur Ausdehnung der zweidimensionalen Strömung ${ }^{8}$.

- Letzteres gilt auch für die vierte von Lee getroffene Annahme. Sofern die horizontale Ausdehnung sehr viel größer ist als die Referenzhöhe, scheint die Näherung plausibel.

Damit widerlegt Finnigan insbesondere die ersten beiden getroffenen Annahmen von Lee, während letztere im Hinblick der von Lee genannten Ursachen für advektive Prozesse (mesoskalige Zirkulationszellen und nächtliche Kaltluftabflüsse) weiterhin plausibel erscheinen.

\subsubsection{Ergänzung der Stoffbilanzgleichung nach Aubinet (2003)}

In der Folgezeit wurde Gleichung (2.5) unter Berücksichtigung o.g. Kritik in einigen Studien zur Bestimmung des Nettoökosystemaustauschs verwendet [Lee98, PBMW00, BFW ${ }^{+}$00]. Die Vorteile des Lee'schen Ansatzes sind nicht von der Hand zu weisen. Er basiert auf einer Ein-Punkt-Messung über der Vegetation und der Messung des $\mathrm{CO}_{2}$-Konzentrationsprofils. Beides Messungen, die in der Regel standardmäßig durchgeführt werden.

Ein entscheidender Kritikpunkt betrifft die Vernachlässigung der horizontalen Advektion (Annahme 2). Deshalb schlägt Aubinet et al. [AHY03] vor, die horizontale Advektion in dem Lee'schen Ansatz wie folgt zu ergänzen:

\footnotetext{
${ }^{8}$ Auch aktuellere Feldexperimente scheinen diese Ausführungen $\mathrm{zu}$ bestätigen $\left[\mathrm{WDC}^{+} 05\right]$.
} 


$$
\begin{gathered}
\int_{0}^{z_{r}} S d z=\int_{0}^{z_{r}} \frac{\partial \bar{C}}{\partial t} d z+\left(\overline{w^{\prime} C^{\prime}}\right)_{r}+\bar{w}_{r}\left(\bar{C}_{r}-\langle\bar{C}\rangle\right)+\int_{0}^{z_{r}} u \frac{\partial \bar{C}}{\partial x} d z . \\
I \quad I I I \quad I V
\end{gathered}
$$

$I \quad$ : Quellen- bzw. Senkenterm von $\mathrm{CO}_{2}$

II : Speicherfluss

III : turbulenter Fluss [AGI $\left.{ }^{+} 00\right]$ (EuroFlux, FLUXNET)

$I V$ : vertikale Advektion $\left[\mathrm{BFW}^{+}\right.$00, Fin99, Lee98, PBMW00]

$V$ : horizontale Advektion [AHY03, FBV03]

Diese Gleichung hat sich innerhalb der CarboEurope-IP Advektionsgruppe (Spoleto 2004) etabliert und kann als weitgehend akzeptiert angesehen werden. Sie bedarf verglichen mit Gleichg. (2.5) zusätzlich der Messung eines horizontalen Wind- und Konzentrationsprofils.

Für die ersten 3d-Setups (Tharandt (Deutschland, 2000-2005), Renon (Italien, 2005), Wetzstein (Deutschland, 2006)) wurde sie um einen weiteren Term entsprechend $V$ quer Hauptwindrichtung ergänzt $\left[\mathrm{ABC}^{+} 05\right.$, FBV03].

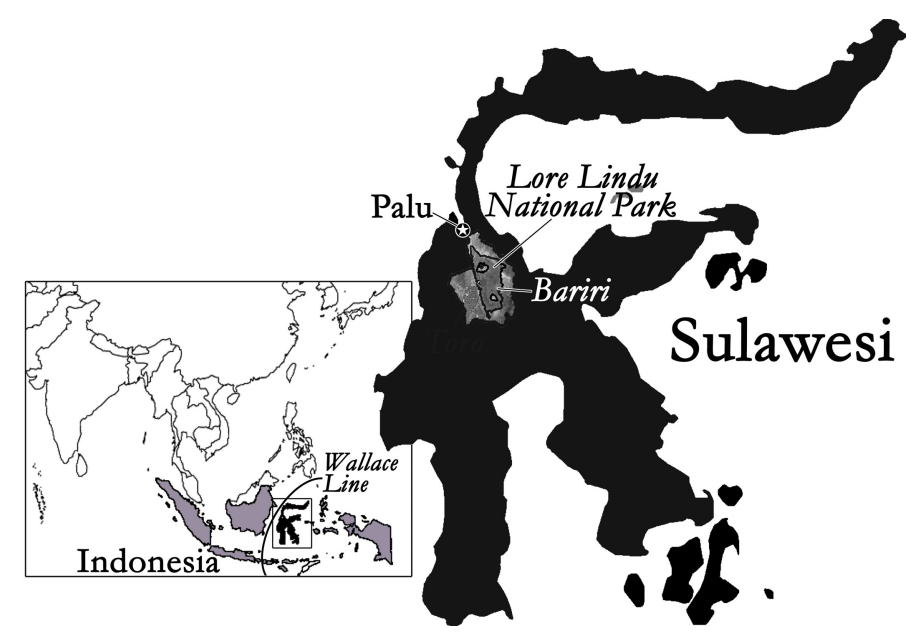

Abbildung 2.3: Das Untersuchungsgebiet auf der Insel Sulawesi/Indonesien östlich der Wallace Line. Quelle: [Die07] (modifiziert)

\subsubsection{Beschreibung des Messgeländes}

Das Untersuchungsgebiet ist als Teil des Lore Lindu Nationalparks (LLNP) in Zentral Sulawesi, Indonesien, nahe des Dorfes Bariri $\left(1^{\circ} 39.476^{\prime} S 120^{\circ} 10.409^{\prime} E\right)$ gelegen (vergl. Abb. 2.3). Der Messturm steht auf einer leichten Reliefwelle in 
$1416 m$ Höhe ü. NN, die sich in nordöstliche Richtung erstreckt (vergl. Abb. 2.4). Nach südwestlichen Richtungen steigt das Gelände nach einer Entfernung von etwa $800 \mathrm{~m}$ bis etwa $1650 \mathrm{~m}$ stark an. Die relative Hangneigung um den Turmstandort herum beträgt innerhalb eines Radius von $600 \mathrm{~m}$ in alle Richtungen weniger als $7 \%$.

In östlichen Richtungen wechselt die Vegetation nach etwa $1200 m-2500 m$

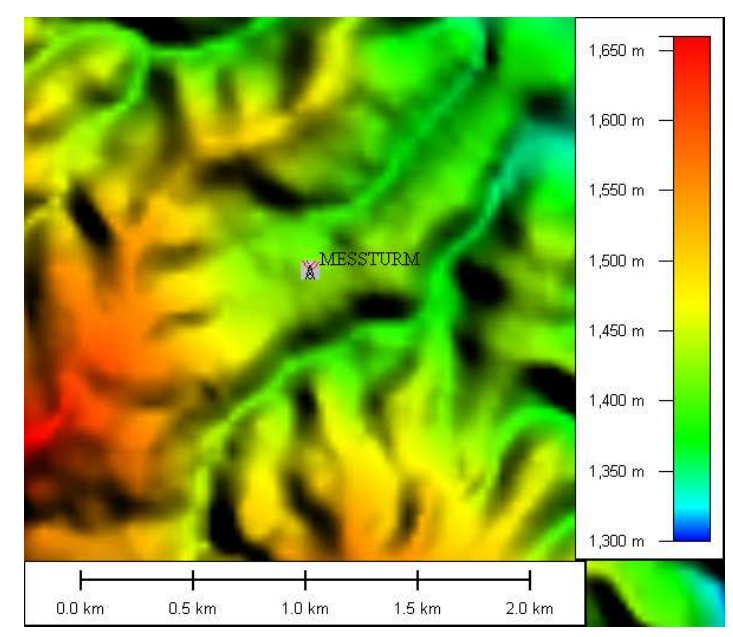

Abbildung 2.4: Geländerelief am Turmstandort Bariri. Die Position des Messturmes ist in der Mitte der Abbildung gekennzeichnet. (Entsprechend der Konvention weist Nord in Richtung oberer Bildrand.)
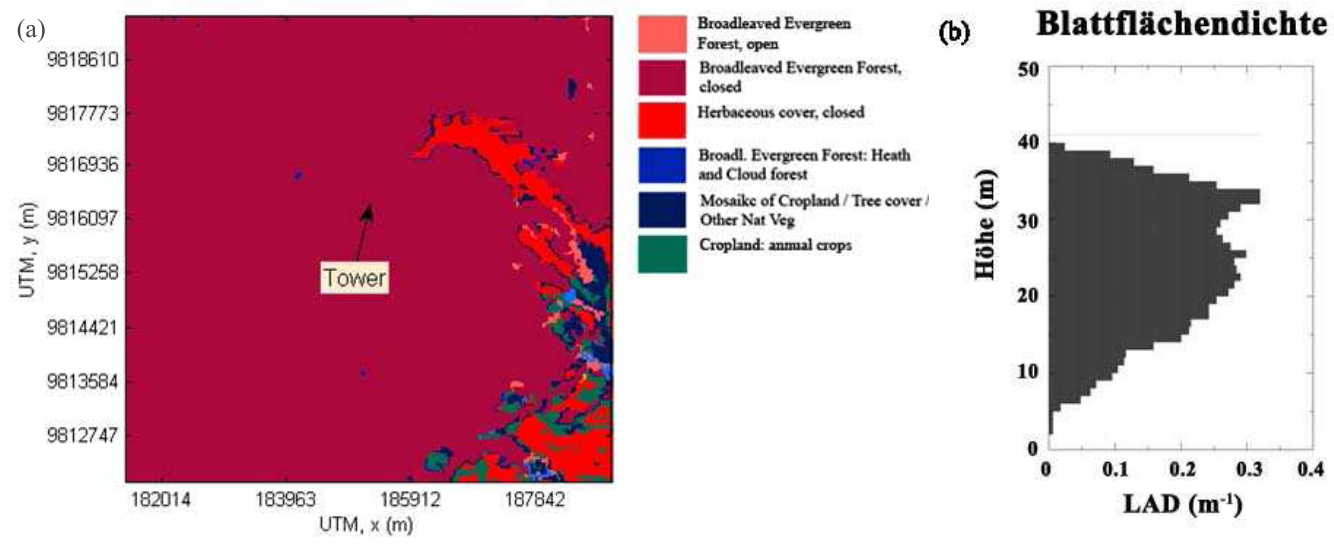

Abbildung 2.5: (a) Vegetationskarte im UTM-Koordinatensystem für das Messgebiet. (b) Darstellung Blattflächendichte (Leaf Area Density) für das geschlossene Waldgebiet um den Turmstandort herum. 
in eine Graslandschaft (vergl. Abb. 2.5 a). Die Vegetation besteht aus etwa 88 Baumarten pro Hektar, wovon etwa 29\% Castanopsis accuminatissima, 18\% Canarium vulgare Leenh. und 9.5\% Ficus Spec. gezählt wurden (Grote S., nichtpublizierte Daten). Weiter ergab die Bestandesaufnahme 550 Bäume pro ha mit einem Brusthöhendurchmesser $>0.1 \mathrm{~m}$ und über 5500 Bäume pro ha mit kleinerem Brusthöhenumfang. Die Baumhöhen betragen am Turmstandort im Schnitt etwa 33m. Der einseitige Blattflächenindex wurde anhand hemisphärischer Fotografien auf ca. 7 festgelegt [Die07]. Abbildung 2.5 (b) zeigt das Vertikalprofil der Blattflächendichte (Leaf Area Density) des Bestandes.

\subsubsection{Messturm und Instrumentierung}

Alle Messungen wurden von einem begehbaren $70 m$ hohen Messturm (Abb. 2.6 a) aus durchgeführt. Zur Messung des Temperatur- und des Windprofils wurden auf drei Ebenen über und zwei Ebenen innerhalb des Bestandes
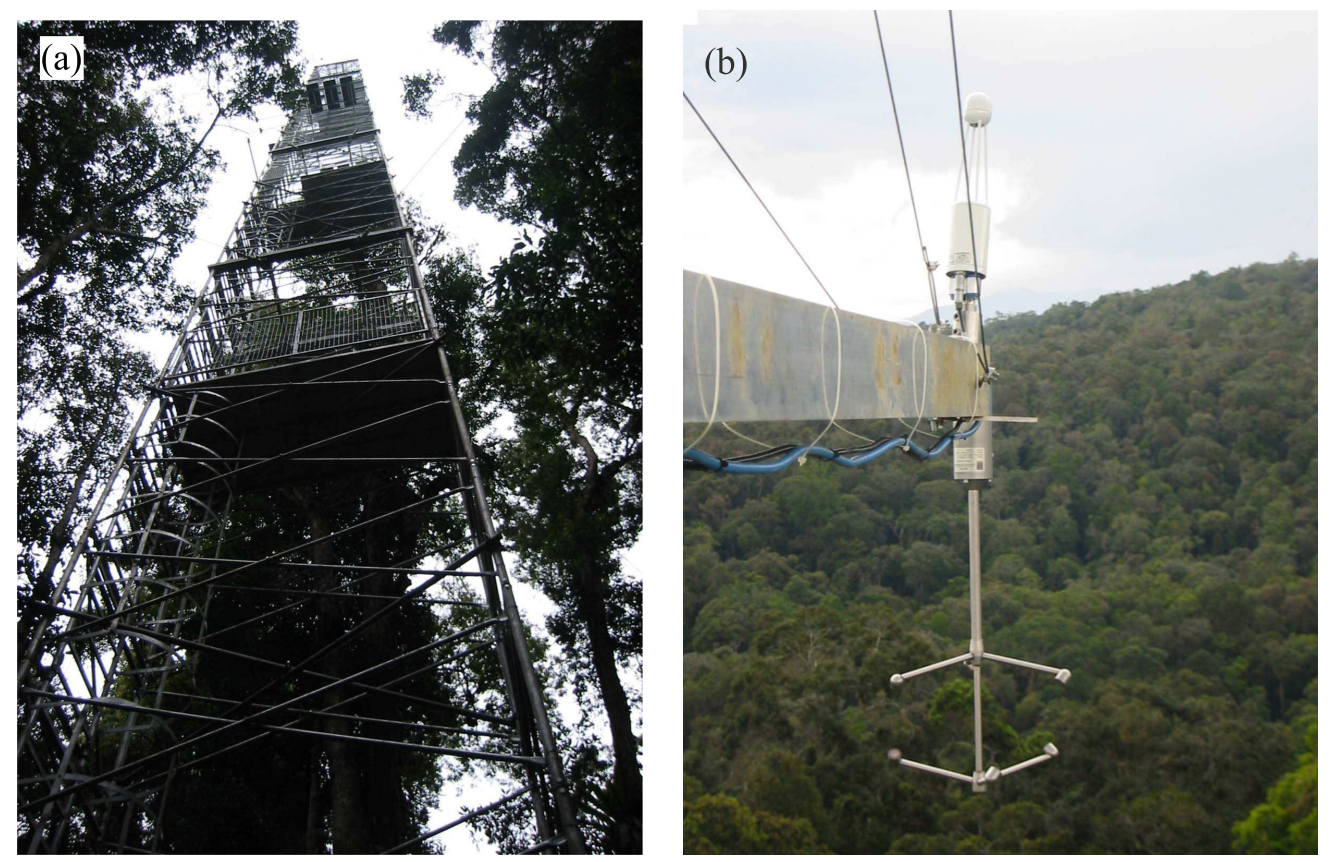

Abbildung 2.6: Bild (a) zeigt die $70 m$ hohe Turmkonstruktion. Kurz oberhalb des Waldbestandes befindet sich die Fotovoltaikanlage zur Stromversorgung (oberer Bildrand). Das Eddy-Kovarianz Messsystem (b) setzt sich aus aus einem Ultraschallanemometer USA-1 der Firma METEK und einem "OpenPath"-Infrarot-Gasanalysator (IRGA) der Firma LICOR zusammen. 
(vergl.. Abb. 2.7 a) ventilierte Temperatur- und Feuchtegeber in Strahlungsschutzhütte sowie Schalenanemometer (Friedrichs Co., Hamburg, Deutschland) verwendet. Lang- und kurzwellige Strahlung über und unterhalb der Vegetation wurde mit CM6B- und CG1-Sensoren der Firma Kipp \& Zonen (Delft, Niederlande) durchgeführt. Niederschlagsmessungen wurden mittels Regensammler mit Wipptechnik vom Hellmann Typ ganz oben auf dem Messturm und an einer 1-2km entfernt gelegenen meteorologischen Bodenstation durchgeführt. Das verwendete Messsystem zur Bestimmung der turbulenten Flüsse besteht aus einem Ultraschallanemometer (USA-1, METEK, Elmshorn, Deutschland) und einem Infrarot - Gasanalysator mit offener Messstrecke (IRGA, LI-COR 7500, Lincoln, Nebraska, USA) [Lic03] (vergl. Abb $2.6 \mathrm{~b})$.
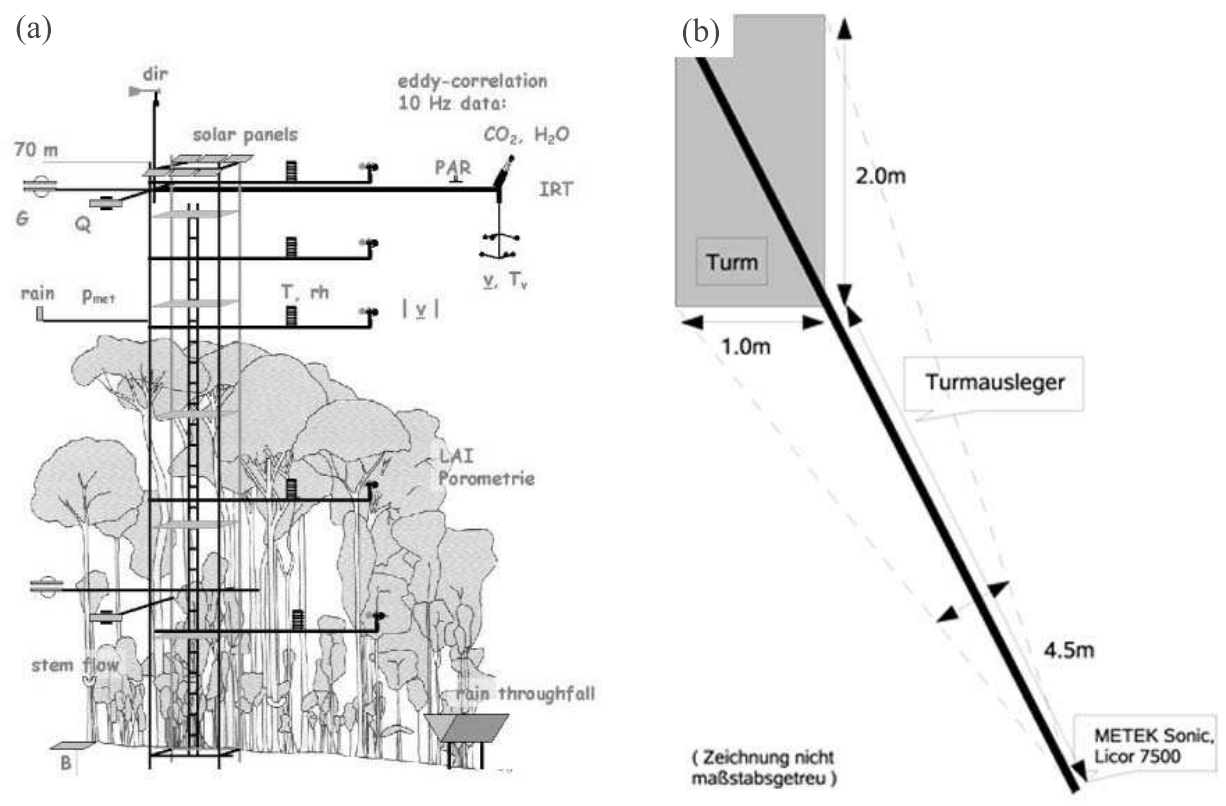

Abbildung 2.7: Schematische Darstellung (a) des Messturms am Standort Bariri. Folgende Messgrößen wurden aufgezeichnet: $U$, die Windgeschwindigkeit respektive -richtung, $T_{a}$, die Lufttemperatur, $H_{r}$, die rel. Feuchte, $R_{k}$ und $R_{l}$, die kurz- und die langwellige Strahlung. Das EK-Messystem für den kontinuierlichen Messbetrieb befindet sich $48 \mathrm{~m}$ über Grund an einem (b) $4.5 m$ langen Ausleger, welcher nach Richtung SSO zeigt. Mit dem nichtstationären Messsystem wurden nacheinander in $56 \mathrm{~m}$ Höhe und in $48 \mathrm{~m}$ Höhe (vis-a-vis des stationären Systems) Vergleichsmessungen durchgeführt (nicht dargestellt). 
Die Analogsignale des Gasanalysators werden zum Ultraschallanemometer transferiert, dort intern digitalisiert und anschließend über eine serielle Schnittstelle (RS-232) auf einem industriellen Mini-PC gespeichert.

\subsubsection{Das Ultraschallanemometer (USA-1, METEK)}

Das Messprinzip aller Ultraschallanemometer besteht aus der Laufzeitmessung von 10-20 $\mathrm{Hz}$ Ultraschallimpulsen entlang einer Messstrecke $d$, die von zwei gegenüberliegenden Sender/Empfänger -Elementen aufgespannt wird. Dabei wird ausgenutzt, dass die Schallgeschwindigkeit relativ zum Trägermedium (Luft) einen allein von diesem abhängigen Wert aufweist. Folglich breiten sich die Schallwellen relativ zum Messgerät, an dem das Trägermedium vorbeiströmt, in Abhängigkeit von der Richtung unterschiedlich schnell aus. Mathematisch ergibt sich die Signalgeschwindigkeit aus der vektoriellen Addition von Schall- und Strömungsgeschwindigkeit des Trägers:

$$
\begin{aligned}
t_{1} & =\frac{d}{c+u}, \quad t_{2}=\frac{d}{c-u}, \\
& \Rightarrow u=\frac{d}{2}\left(\frac{1}{t_{1}}-\frac{1}{t_{2}}\right),
\end{aligned}
$$

wobei $t_{1}$ respektive $t_{2}$ die Laufzeiten des Schallimpulses zur Laufzeitdifferenzbildung (Hin- und Rückweg), $\underline{u}$, die Windgeschwindigkeitskomponente parallel zu Messstrecke $x$ und $c$, die Schallgeschwindigkeit in Luft bezeichnet. Schließlich sind die Schallstrecken auf drei linear unabhängige Richtungen zu erweitern, sodass alle Komponenten des Windvektors erfasst werden.

Eine ausführlichere Beschreibung des 3d-Ultraschallanemometers (USA-1) ist im Manual der Firma METEK oder beispielsweise in [Fal04] zu finden.

\subsubsection{Der "Open-Path" Infrarotgasanalysator (IRGA 7500, LI- COR)}

Das elektromagnetische Spektrum enthält im infraroten Bereich starke Absorptionsbanden von Wasserdampf und Kohlendioxid. Daher bietet das Absorptionsvermögen der in der Luft enthaltenden Moleküle eine sichere und spezifische Möglichkeit, deren Konzentration zu messen. Das Gerät selber zeichnet sich durch eine offene Messstrecke (open path) und der für EKMessungen erforderlichen nominellen Antwortzeit von $\leq 0.1 s$ aus.

Zum Funktionsprinzip: Nach dem Skalierungsgesetz von Jaimeson (et al. 
1963) [Lic03] hängt das Verhältnis von Absorptionsvermögen und Partialdruck in einem bestimmtem Frequenzbereich wie

$$
\frac{\alpha_{i}}{P_{e i}}=h_{i}\left(\frac{a_{i}}{P_{e i}}\right)
$$

ab. Demnach lässt sich das Absorptionsvermögen $\alpha_{i}$ eines Gases $i$ beschreiben durch $a_{i}$, der Menge des als Absorber wirkenden Gases und $h_{i}$, einer Funktion von $a_{i} / P_{e i}$. $P_{e i}$ ist der Partialdruck des $i$.ten Gases. Nach Einführung der Teilchenzahldichte $\rho_{i}$ und der Messstreckenweglänge $\lambda$ folgt mit $a_{i}=\lambda \rho_{i}$

$$
\rho_{i}=P_{e i} f_{i}\left(\frac{\alpha_{i}}{P_{e i}}\right)
$$

mit $f_{i}$, der eigentlichen Kalibrierfunktion. Anschließend wird eine Reihe bekannter Teilchenzahldichten $\rho_{i}$ durchgemessen. $f_{i}$ ergibt sich dann als Kalibrierungskurve durch die Messpunkte $\rho_{i} / P_{e i}$ gegen $\alpha_{i} / P_{e i}$.

Andererseits gilt:

$$
\alpha_{i}=1-\tau_{i}=1-\frac{A_{i}}{A_{i 0}},
$$

wobei $\tau_{i}$ den Transmissionsgrad und $A_{i}$, die transmittierte Strahlungsleistung durch den Absorptionsbereich des Gases $i$ beschreibt. $A_{0}$ gibt die transmittierte Strahlungsleistung einer Referenzwellenlänge an, die das Gas $i$ nicht absorbiert. Dazu werden $A_{i}$ und $A_{i 0} 152$ mal in der Sekunde gemessen.

Nach Einsetzen von Gleichg. 2.15 in Gleichg. 2.14 ergibt sich die vollständige Gleichung zur Berechnung der Teilchenzahldichte aus der Absorption:

$$
\rho_{i}=P_{e i} f_{i}\left(\left[1-\frac{A_{i}}{A_{i 0}} z_{i}\right] \frac{S_{i}}{P_{e i}}\right)
$$

Zwei Terme $z_{i}$ und $S_{i}$, "Zero" und "Span" sind zur nachträglichen Kalibrierung hinzugekommen. Obwohl der Detektor und die Filter im LI-7500 temperaturgeregelt sind, ist der Detektor nicht vollkommen unempfindlich gegenüber Veränderungen der Umgebungstemperatur (ca. 0.1-0.2ppm pro Grad ${ }^{\circ} \mathrm{C}$ bei $\mathrm{CO}_{2}$ und $0.01 \mathrm{mmol} / \mathrm{mol}$ pro Grad ${ }^{\circ} \mathrm{C}$ bei $\mathrm{H}_{2} \mathrm{O}$ ). Etwaige Fehler sind direkt mit der sog. "cooler control" Spannung verbunden, die mitgemessen wird. Es wird daher empfohlen, nach 4-5 Monaten betrieb über die Beziehung $z_{i}=Z_{i 0}+Z_{i} V_{d}$ eine Software-Feineinstellung vorzunehmen. $V_{d}$ ist die Detektor cooler Spannung und $Z_{i}$ respektive $Z_{i 0}$ werden bei der Kalibrierung ermittelt.

In der Praxis werden zunächst die Zero Werte für $\mathrm{CO}_{2}\left(z_{C}\right)$ und $\mathrm{H}_{2} \mathrm{O}\left(z_{W}\right)$ ermittelt, indem ein kontinuierlicher Strom trockener $\left(\alpha_{W}=0\right)$ und $\mathrm{CO}_{2}$-freie Luft $\left(\alpha_{C}=0\right)$ durch die optische Messstrecke geführt wird. Danach werden 
Tabelle 2.1: Tabelle der durchgeführten Kalibrierungen der beiden IRGA's (LICOR-7500), Ref.:[Lic03]. Das stationäre Gerät ist als Nr. 1 und das mobile als Nr. 2 bezeichnet. (Nähere Erläuterungen: siehe Text.)

\begin{tabular}{lrrrrr}
\hline Datum & Gerät & $z_{C}$ & $S_{C}$ & $z_{W}$ & $S_{W}$ \\
\hline \hline 29.Sep $/ 2004$ & 1 & 0.8846 & 1.0027 & 0.7049 & 0.8594 \\
30.Sep $/ 2004$ & 1 & 0.8948 & 1.0509 & 0.6943 & 0.9151 \\
01.Okt $/ 2004$ & 2 & 0.9765 & 0.9756 & 0.6483 & 0.8994 \\
02.Okt/2004 & 2 & 0.9792 & 1.2472 & 0.6501 & 1.2250 \\
\hline
\end{tabular}

die Span Werte für $\mathrm{CO}_{2}\left(S_{C}\right)$ respektive $\mathrm{H}_{2} \mathrm{O}\left(S_{W}\right)$ festgelegt. Dazu wird für $S_{C}$ Gas mit bekannter Teilchenzahldichte $\rho_{C}$ oder Mischungsverhältnis (z.B. 500-1000ppm) verwendet. Um $S_{W}$ zu bestimmen, kann ein Taupunktgenerator verwendet werden. Dabei ist zur Vermeidung von Kondensation darauf zu achten, den Taupunkt einige Grad unterhalb der Umgebungstemperatur zu wählen.

2.1.4.2.1 Querempfindlichkeit Die IR-Absorptionsbanden von Kohlendioxid und Wasserdampf überlappen sich leicht. Zur Berechnung der tatsächlichen Kohlendioxid- respektive Wasserdampfabsorption muss der Ausdruck für den Transmissionsgrad (Gleichg. 2.15) leicht modifiziert werden (vergl. [Lic03]), um Querempfindlichkeiten zu berücksichtigen. So gilt für den Absorptionsgrad des Gases $i$

$$
\alpha_{i}=1-\left(\frac{A_{i}}{A_{i 0}}+X_{j i}\left(1-\frac{A_{j}}{A_{j 0}}\right)\right)
$$

wobei $X_{j i}$, die Querempfindlichkeit von Gas $j$ auf Gas $i$ bezeichnet (bei der Kalibrierung festgelegt).

\subsubsection{Korrekturen}

\subsubsection{Webb, Pearman und Leuning (WPL) Term}

1980 wurde von Webb, Pearman und Leuning ein in der Literatur oft als "WPL Korrektur" bezeichneter mathematischer Ausdruck zur Berechnung turbulenter Skalarflüsse abgeleitet. Da es sich nicht wirklich um eine Korrektur handelt, wird heutzutage nur noch vom WPL-Term gesprochen. 
Um diesen Term näher zu erläutern, scheint es sinnvoll, zunächst die turbulente Massenflussdichte herzuleiten. Ein Massenfluss durch eine gedachte horizontale Ebene lässt sich beschreiben durch eine Funktion der Geschwindigkeit, der Dichte und des relativen Anteils des betrachteten Skalars eines Luftpaketes. Mit $\rho_{a}$, der Dichte trockener Luft, $\rho_{c}$ der Dichte des Skalars und $s$ dem Mischungsverhältnis $\left(\rho_{c} / \rho_{a}\right)$ ergibt sich nach Mittlung:

$$
F_{c}=\overline{\rho_{a}} \overline{w s} .
$$

Anschließende Anwendung der Zerlegung nach Reynolds (siehe Abschn. 2.1.2) führt zu acht Termen. Drei Terme sind sofort identisch Null. Und da der Fluss trockener Luft $\overline{w \rho_{a}}=\overline{w^{\prime} \rho_{a}^{\prime}}+\bar{w} \overline{\rho_{a}}$ per Definition Null ist und in der Folge Terme dritter und vierter Ordnung vernachlässigbar klein sind, verbleibt:

$$
F_{c}=\overline{\rho_{a}} \overline{w^{\prime} s^{\prime}}
$$

Nun werden Messungen nach der EK-Methode in den allermeisten Fällen mithilfe eines Ultraschallanemometers und eines Infrarot-Gasanalysators (IRGA) durchgeführt. IRGA's, wie z.B. der verwendete, messen jedoch nicht "parts per million" (ppm), sondern via Infrarot-Spektroskopie die Transmission von Licht durch ein Probenvolumen (vergl. Abschn. 2.1.4.2), d.h. die molare Dichte $\left(\rho, \rho^{\prime}\right.$ in $\left.\mathrm{mol} / \mathrm{m}^{3}\right)$ :

$$
F_{c}=\overline{w \rho_{c}}=\overline{w^{\prime} \rho_{c}^{\prime}}+\bar{w} \bar{\rho}_{c}
$$

Dass die Gleichungen 2.18 und 2.19 übereinstimmen, lässt sich leicht mithilfe der Regeln einfacher Differentialoperatoren zeigen ${ }^{9}$. Somit zeigt sich: Der Fluss aus $\overline{\rho_{a}} \overline{w^{\prime} s^{\prime}}$ entspricht der Summe aus der Kovarianz $\overline{w^{\prime} \rho_{c}^{\prime}}$ und einem gemittelten Driftterm $\bar{w} \bar{\rho}_{c}$. In der Regel (horizontal homogenes Gelände) ist die mittlere vertikale Geschwindigkeit $\bar{w}$ sehr klein, z.T. unter dem Auflösungsvermögen des Ultraschallanemometers. Die skalare molare Dichte kann jedoch sehr hohe Werte annehmen (z.B. $580 \mathrm{mg} \mathrm{m}^{-3}$ für $\mathrm{CO}_{2}$ ), sodass das Produkt zu signifikanter Flussdichte führen kann.

Um Gleichung 2.19 weiter auszuführen, ist zu berücksichtigen, dass die Luft unter Feldbedingungen feucht $\left(\rho_{\nu}\right)$ ist. Aus dem Gesetz der Partialdrücke und dem Gasgesetz ergibt sich

$$
\frac{\rho_{a}}{m_{a}}+\frac{\rho_{\nu}}{m_{\nu}}=\frac{p}{R T}
$$

\footnotetext{
${ }^{9}$ Aus $\delta s\left(\rho_{c}, \rho_{a}\right)=\frac{\partial s}{\partial \rho_{c}} \delta \rho_{c}+\frac{\partial s}{\partial \rho_{a}} \delta \rho_{a}$ ergibt sich ein Ausdruck für kleine Fluktuationen $s^{\prime}$, der eingesetzt in $\overline{w^{\prime} s^{\prime}}$ mit $\overline{w^{\prime} \rho_{a}^{\prime}}=-\bar{w} \overline{\rho_{a}}$ unmittelbar auf Gleichg. 2.19 führt.
} 
und nach Entwicklung:

$$
\frac{\rho_{a}^{\prime}}{m_{a}}+\frac{\rho_{\nu}^{\prime}}{m_{\nu}}=-\frac{p}{R \bar{T}} \frac{T^{\prime}}{\bar{T}}+\ldots
$$

Auflösen nach den Dichtefluktuationen trockener Luft ergibt

$$
\rho_{a}^{\prime}=-\frac{m_{a}}{m_{\nu}} \rho_{\nu}^{\prime}+\overline{\rho_{a}}\left(1+\frac{\overline{\rho_{\nu}} m_{a}}{\overline{\rho_{a}} m_{\nu}}\right) \frac{T^{\prime}}{\bar{T}}
$$

womit sich ein neuer Ausdruck für die vertikale Geschwindigkeit $\bar{w}=-$ $\overline{w^{\prime} \rho_{a}^{\prime}} / \overline{\rho_{a}}$ angeben lässt:

$$
\bar{w}=-\frac{m_{a}}{m_{\nu}} \frac{\overline{w^{\prime} \rho_{\nu}^{\prime}}}{\overline{\rho_{a}}}+\left(1+\frac{\overline{\rho_{\nu}} m_{a}}{\overline{\rho_{a}} m_{\nu}}\right) \frac{\overline{w^{\prime} T^{\prime}}}{\bar{T}} .
$$

Indem Gleichung 2.23 schließlich in Flussgleichung 2.19 eingesetzt wird, ergibt sich der WPL-Term für den Open-path-Sensor:

$$
F_{c}=\overline{w^{\prime} \rho_{c}^{\prime}}-\frac{m_{a}}{m_{\nu}} \frac{\overline{\rho_{c}}}{\overline{\rho_{a}}} \overline{w^{\prime} \rho_{\nu}^{\prime}}+\left(1+\frac{\overline{\rho_{\nu}} m_{a}}{\overline{\rho_{a}} m_{\nu}}\right) \frac{\overline{\rho_{c}}}{\bar{T}} \overline{w^{\prime} T^{\prime}} .
$$

Einschränkend ist zum Schluss zu bemerken, dass etwaige Dichtefluktuationen nicht berücksichtigt wurden. Nach [ML02] sind auf Druckfluktuationen zurückzuführende Korrekturen vor allem dann signifikant, wenn in sehr hohen Lagen verbunden mit großen Windgeschwindigkeiten gemessen wird. Da in den Tropen in der Regel schwache Winde vorherrschen, erscheint der Ausdruck Gleichg. 2.24 vertretbar.

\subsubsection{Sensibler Wärmestrom nach Schontanus}

Zur Bestimmung des sensiblen Wärmestroms wird die sogenannte virtuelle Temperatur $T_{V}=T(1+0.61 q)$ verwendet. Die virtuelle Temperatur trägt der dichteverändernden Eigenschaft des Wasserdampfs Rechnung. Sie entspricht derjenigen höheren Temperatur, die hypothetisch trockene Luft haben müsste, um die gleiche Dichte zu besitzen, wie feuchte Luft bei einer niedrigeren Realtemperatur und gleichem Druck.

Ein Ultraschallanemometer misst die der virtuellen Temperatur gut entsprechenden akustische Temperatur $T_{S}$. Nach [SB83] sind die Schalltemperaturfluktuationen derart zu korrigieren, dass der Einfluss der Luftfeuchte berücksichtigt wird. Außerdem würde die Schallgeschwindigkeit durch laterale Impulsflüsse gestört. Für den korrigierten sensiblen Wärmestrom ergibt sich 


$$
\overline{w^{\prime} T_{a}^{\prime}}=\overline{w^{\prime} T_{S}^{\prime}}-0.51 \overline{w^{\prime} q_{a}^{\prime}} \bar{T}_{a}+\frac{3 T_{a}}{2 c_{p}^{2}}\left(\bar{u} \overline{w^{\prime} u_{c}^{\prime}}+\bar{v} \overline{w^{\prime} v_{c}^{\prime}}\right),
$$

wobei $c_{p}$, die spezifische Wärme bei konstantem Druck ist. Mit dem zweiten Term auf der rechten Seite wird die Feuchte, mit dem dritten der laterale Impulsfluss berücksichtigt.

Dabei stellt Formel 2.25 nicht die von Schontanus ursprünglich abgeleiteten Ausdruck für den sensiblen Wärmestrom dar. Sie wurde von Liu et al. [LPF01] leicht modifiziert, da sich die Methode zur Berechnung der Schalltemperatur bei den modernen Ultraschallanemometern geändert hat.

Bei labiler Schichtung kann die Feuchtekorrektur und bei nahezu neutraler Schichtung die Querwindkorrektur einen bis zu 20 prozentigen Einfluss auf den sensiblen Wärmestrom haben. Sonst tragen die einzelnen Terme mit Korrekturen von bis zu $5 \%$ oder weniger bei (nach Longdoz, pers. Gespräch $\left.\left[\mathrm{BEF}^{+} 06\right]\right)$.

\subsubsection{Das Planar-Fit Verfahren}

Im Fall eines idealen Standorts, der sich durch ebenes Gelände und horizontal homogen verteilte Vegetation kennzeichnen lässt, ist aufgrund einfacher Kontinuitätsannahmen a priori von dem Verschwinden der mittleren Vertikalkomponente der Geschwindigkeit auszugehen. Demzufolge definierten Tanner \& Thurtell [TT69] ein rechtwinkliges Koordinatensystem, dessen $x$-Achse parallel zur mittleren Hauptströmungsrichtung verläuft. Die $z$-Achse ist entsprechend senkrecht zur $x$-Achse angeordnet und weist von der Unterlage weg nach oben. Weiterhin wird angenommen, dass keine Korrelation zwischen den lateralen respektive den vertikalen Geschwindigkeitsfluktuationen existiert $\left(\overline{v^{\prime} w^{\prime}}=0\right)$. Eine ausführlichere Beschreibung ist im Originalreport und in [McM88] zu finden.

Mit der Ausweitung mikrometeorologischer Messungen auf komplexere Standorte und Messkampagnen, die ein Jahr und weit länger andauern, wurden einige Nachteile der o.g. Methode offenbar. So hat es sich z.B. als problematisch herausgestellt, für jede Mittlungsperiode definitionsgemäß $\bar{w}=0$ zu setzen. Dadurch besteht die Gefahr, das Bezugssystem zu "überdrehen" (over-rotation). Eine typische mittlere Windgeschwindigkeit von $2 \mathrm{~m} / \mathrm{s}$ und ein Instrumenten-Offset der Vertikalkomponente der Geschwindigkeit von $5 \mathrm{~cm} / \mathrm{s}$ führen beispielsweise zu einer Überdrehung von $1.5^{\circ}$. In der Folge kann es zu einem systematischen Überschätzen der zeitintegrierten Flüsse 
kommen ${ }^{10}$ [LL04].

Weitere Ursachen für ein fehlerhaft ausgerichtetes Koordinatensystem hängen mit der Beeinflussung der Strömung durch die tragenden Teile des Anemometers, durch die Turmaufbauten und den Instrumententurm selber zusammen. Durch sie wird die Strömung windrichtungsabhängig beeinflusst. Zuletzt sei

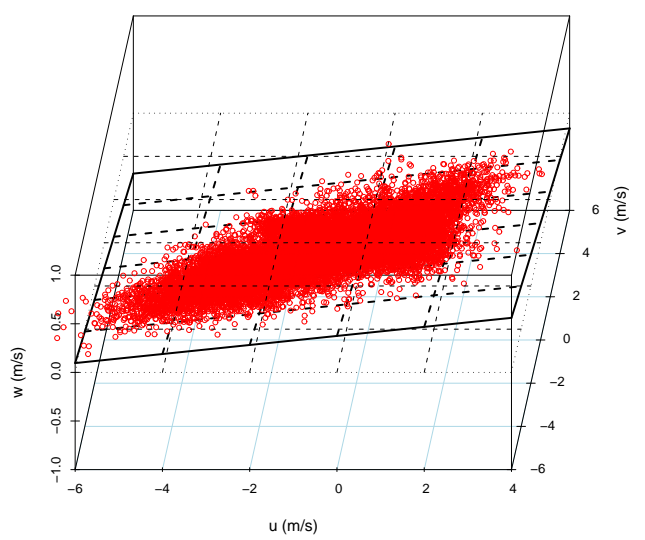

Abbildung 2.8: Bestimmung des Koordinatensystems nach dem Planar-Fit Verfahren (Illustration). $u, v$ und $w$ bezeichnen die mittleren Windkomponenten, durch welche die Referenzebene festgelegt wurde, Ref.: [WOS01].

der Verlust von Information erwähnt. In Kapitel 2.1.2.1 wurde ausführlich auf die komplexen meteorologischen Bedingungen hingewiesen, welche mit ausgeprägt unebenem Relief und konvektiven Prozessen verbunden sind. Eine verschwindende Vertikalkomponente der Geschwindigkeit ist insofern nicht zu erwarten. Auch die Annahme $\left(\overline{v^{\prime} w^{\prime}}=0\right)$ ist unter diesen Umständen nicht haltbar [Fin04].

Mithilfe neuerer Methoden zur Festlegung des Bezugssystems [Lee98, WOS01, PBM00] wird versucht, die Nachteile des sog. natürlichen Koordinatensystems zu überwinden. Weitesgehend durchgesetzt hat sich heute die sogenannte "planar fit" Methode [WOS01]. Dieses Verfahren basiert auf der Idee, dass ein allein vom Geländerelief abhängiges mittleres Windfeld existiert, durch welches sich ein Koordinatensystem sinnvoll definieren lässt. Die einzelnen Schritte zur Bestimmung des neuen Koordinatensystems sind die folgenden:

\footnotetext{
${ }^{10}$ Anhand des Beispiels wird auch klar, dass das Problem vor allem bei niedrigen Windgeschwindigkeiten zum Tragen kommt.
} 
- Bestimmung der Messperiode, in der sich die Anemometerposition relativ zum Gelände nicht geändert hat.

- Durchführung einer linearen Regression $\overline{w_{m}}=b_{0}+b_{1} \overline{u_{m}}+b_{2} \overline{v_{m}}$, um die mittlere Strömungsebene zu definieren (siehe Abb. 2.8), wobei $b_{0}, b_{1}$ und $b_{2}$ die Regressionskoeffizienten und $u_{m}, v_{m}$ und $w_{m}$ die mittleren Geschwindigkeitskomponenten im Instrumenten-Koordinatensystem darstellen.

- Festlegung der Rotationswinkel $\alpha, \beta$ und $\gamma$ durch die Regressionskoeffizienten $b_{1}$ und $b_{2}$, wie es in [WOS01] beschrieben wird.

- Projektion der Geschwindigkeitskomponenten und der Kovarianzen in das neue Koordinatensystem.

Mit der Planar-Fit-Methode wird zum einen der o.g. Instrumenten-Offset eleminiert. Indem die Daten aller respektive größerer Sektoren von Windrichtungen verwendet werden, kann das Bezugssystem zum anderen statistisch sicherer ermittelt werden. Zu beachten ist, dass für die Festlegung der Referenzebene nur Messperioden annähernd neutraler Schichtung verwendet werden sollten [Fin99]; allein unter diesen Bedingungen sollte die Strömung dem Geländerelief folgen.

\subsubsection{Beurteilung der Datenqualität}

\subsubsection{Der Stationaritätstest}

Der Stationaritätstest ist von grundlegender Bedeutung. Er stellt eines der wichtigsten Kriterien zur qualitativen Beurteilung von Messungen nach der EK-Methode dar.

Stationarität bedeutet, dass die statistischen Eigenschaften einer Zeitreihe zeitlich konstant sind. Instationaritäten der Flüsse entstehen vor allem im Zuge sich ändernder Wetterbedingungen oder eines Quellgebietswechsels innerhalb eines Messintervalls. Außerdem sind Situationen, in denen der vertikalen Stoffaustausch durch stabile atmosphärische Schichtung unterdrückt wird, häufig Ursache für Instationaritäten der turbulenten Flüsse. Zur Überprüfung wird die Kovarianz der ursprünglichens Zeitreihe, mit dem Mittel der Kovarianzen kürzerer Unterabschnitte verglichen [FW96]. Im Rahmen dieser Arbeit wurden Unterabschnitte von 5 Minuten Länge verwendet.

Nach Foken \& Wichura [FW96] ist Stationarität gegeben, wenn sich die Kovarianzen um weniger als $\pm 30 \%$ unterscheiden. Dabei ist zu kritisieren, dass die zu vergleichenden Beträge als gleich groß angenommen werden. Denn bei 
der Verwendung kürzerer Zeitreihenabschnitte gehen zwangsläufig energiereiche Perioden des Leistungsspektrums verloren [Ibr01]. Im Rahmen dieser Arbeit werden Kohlendioxidflussbeiträge als stationär gewertet, wenn für das Verhältnis

$$
t_{\text {stat }}=\left|\frac{\mu_{5 \min }-\mu_{30 \min }}{\mu_{30 \min }}\right|
$$

mit $\mu_{30 \text { min }}$, der Kovarianz der ursprünglichen Zeitreihe und $\mu_{5 \min }$, der Kovarianz aus dem Mittel der sechs Unterabschnitte, $t_{\text {stat }}<0.3$ gilt. Steigt das Verhältnis über 0.5 werden die entsprechenden Werte ausgeschlossen [IRK ${ }^{+}$, FW96].

\subsubsection{Integrale Turbulenzcharakteristik}

Integrale Turbulenzcharakteristiken stellen ein Kriterium dar, um festzustellen, ob das turbulente Windfeld voll entwickelt ist. Ist dies nicht der Fall, z.B. weil Hindernisse (Turmaufbauten etc.) oder die Inhomogenität des Geländes zusätzlich Turbulenz erzeugt, kommt es zu Abweichungen zwischen den gemessenen und den modellierten Turbulenzcharakteristiken [Fok03]. Für den Vertikalwind gibt es kaum Abweichungen zwischen den verschiedenen Parametrisierungen. Das Modell zur Beschreibung der Turbulenzcharakteristik für den Vertikalwind lässt sich wie folgt angeben [PD88]:

$$
\frac{\sigma_{w}}{u_{*}}=1.3\left(1-2 \frac{(z-d)}{L}\right)^{1 / 3} \text {. }
$$

Dabei bezeichnet $z$ die Messhöhe, $d$ die Versatzhöhe durch die Vegetation und $L$ die Obukhovlänge. Die mit $u_{*}$ normierte Standardabweichung des vertikalen Windes wird als integrale Turbulenzcharakteristik bezeichnet, weil sie integral über alle Frequenzen den Turbulenzzustand charakterisiert.

\subsubsection{Prozedur der Datennachbearbeitung}

Die Datenerfassung erfolgte mit einer institutseigenen Software der Programmiersprache $C$. Alle halbe Stunde wurden neben den Rohdaten (Auflösung: $10 H z$ ) die zugehörige Standardstatistik (Momente 1. und 2. Ordnung) aufgenommen, konvertiert und zur Reduzierung des Speicherplatzbedarfs in Form von Binärdateien abgespeichert.

Die Nachbearbeitung wurde wie folgt mit der "Post-Processing" - Software $R C P M$ [Mor00, Ibr01] durchgeführt: 
- Entfernung der Ausreißer ("Despiking") mit modifizierter Software von Hojstrup [Hoj93].

- Anwendung einer Windkanalkorrektur [Ble01] für das Ultraschallanemometer USA-1 von METEK.

- Kovarianzmaximierung zur Synchronisierung der Zeitreihen

- WPL-Korrektur [WP80].

- Querwindkorrektur der Schalltemperaturmessung für das USA-1 (METEK) [LPF01].

- Berechnung der 30-Minuten Statistik einschließlich Kovarianzen.

- Test auf Stationarität [FW96].

- Korrektur der Sensorkopftrennung nach Kristensen et al. [KMOW97].

Die $R C P M$ Software wird stetig dem wissenschaftlichen Kenntnisstand angepasst und wurde mehrfach erfolgreich im Rahmen des EUROFLUX Projekts mit anderen Nachbearbeitungssoftwares verglichen $\left[\mathrm{ACE}^{+} 03\right]$.

Neu hinzugekommen ist das Planar-Fit Verfahren. Dieses - sowie einige Aktualisierungen - wurde speziell zur Anfertigung dieser Arbeit geschrieben und getestet. Die Integration in die $R C P M$ Software wurde unterlassen, da das Verfahren in der Regel für definierte Windrichtungsbereiche durchzuführen ist. Neben dem Planar-Fit Verfahren [WOS01] wurde zudem das konventionelle Rotationsverfahren [McM88] zur Festlegung des Koordinatensystems durchgeführt, um etwaige Unterschiede $\mathrm{zu}\left[\mathrm{IRK}^{+}\right]$- der vorangegangenen Veröffentlichung - herauszuarbeiten.

Die berechneten Flüsse ergeben sich aus dem Produkt von Vertikalfluktuation und molarer Dichte der betrachteten Spurengase $\mathrm{CO}_{2}, \mathrm{H}_{2} \mathrm{O}$ respektive der virtuellen ${ }^{11}$ Temperatur. Zusätzlich sind jedoch Temperatur-, Druck- und Feuchteeffekte zu berücksichtigen (Abschn. 2.1.5.1) sowie zur Berechnung des sensiblen Wärmestroms, die sogenannte Schotanus-Korrektur (Abschn. 2.1.5.2).

\subsection{Verfahren zur Datenlückenschließung}

Wie bereits in der Einleitung erwähnt, ist die Kohlenstoffbilanzierung des untersuchten tropischen Hochregenwaldes in Indonesien gerade vor dem Hin-

\footnotetext{
${ }^{11}$ Tatsächlich wird die akustische Temperatur gemessen (siehe Abschn. 2.1.5.2).
} 
tergrund der aktuellen Klimaproblematik von großem Interesse. Informationen über den jährlichen Nettoökosystemaustausch (net ecosystem exchange, $N E E$ ) verschiedenster Vegetationen und aus den verschiedensten Regionen der Welt können wesentlich zum besseren Verständnis beitragen, welche Rolle terrestrische Ökosysteme im globalen Kohlenstoffkreislauf spielen (missing sink). Etwa $22 \%$ der starken Erhöhung der $\mathrm{CO}_{2}$-Konzentration binnen der letzten 200 Jahre gehen auf Landnutzungsänderungen zurück. Dabei weist die Region Süd-Ost Asien die höchsten Abholzungsraten tropischer Regenwälder überhaupt auf [SKBN04].

Zur Berechnung des $N E E$, also des $\mathrm{CO}_{2}$ Austausches zwischen Vegetation und Atmosphäre, hat man sich den Fluss in zwei konkurrierende Anteile aufgeteilt zu denken - einen assimilatorischen und einen respiratorischen. Assimilation ist mit Fotosynthese verbunden, d.h. $\mathrm{CO}_{2}$ wird am Tage während der Vegetationsperiode aufgenommen. Dagegen findet Respiration sowohl bei Tage als auch in der Nacht statt. Sie beinhaltet nicht nur die Atmung fotosynthetisierender Zellen und Pflanzen sondern auch die Atmung von Ästen und Stämmen. Außerdem kommt noch die autotrophe und heteotrophe Atmung hinzu. In dieser Arbeit wird von assimilatorischen Flüssen gesprochen, sofern die Globalstrahlung $\left(R_{G}\right)$ Werte $>10 W m^{-2}$ annimmt. Im anderen Fall werden respiratorische Flüsse definiert.

Zunächst einmal abgesehen von etwaigen advektiven Flüssen (Term $I V$, Gleichg. 2.10), lässt sich der $N E E$ aus dem turbulenten Fluss (Term II, Gleichg. 2.10) und dem Speicherterm (Term III, Gleichg. 2.10) berechnen. Da bis heute am Standort Bariri noch nicht über längere Perioden Speichertermmessungen im Profil durchgeführt werden konnten, wurde zur Abschätzung dieser Messung eine in $\left[\mathrm{HKB}^{+} 94\right]$ beschriebene Methode verwendet. Dazu wird die Änderungsrate der $\mathrm{CO}_{2}$ Konzentration in Messhöhe als Approximation für die Konzentrationsänderung der gesamtem Luftsäule unterhalb des Sensors betrachtet. Ergänzend wurden modellierte und die wenigen gemessenen $\mathrm{CO}_{2}$-Konzentrationsprofile zur Speichertermberechnung herangezogen. Der Konvention folgend werden Flüsse von der Atmosphäre in den Bestand negativ und vom Bestand in die Atmosphäre positiv gezählt. 


\subsubsection{Modellierung der assimilatorischen $\mathrm{CO}_{2}$-Flüsse}

\subsubsection{Lichtsättigungsfunktion nach Michaelis-Menten}

Zur Untersuchung der Nettoassimilation ${ }^{12}$ wurden die $\mathrm{CO}_{2}$-Flüsse zunächst in Klassen relevanter meteorologischer Parameter unterteilt. Nach eingehender Analyse stellten sich zwei Parameter als besonders bedeutsam heraus: die Temperatur und der Wolkenbedeckungsgrad. Dagegen erwiesen sich mögliche saisonal bedingte Veränderungen der Blattphysiologie, die sich im Blattflächenindex (Leaf area index, LAI) widerspiegeln sollten oder Inhomogenitäten bezüglich des Fetch erwartungsgemäß als weniger von Bedeutung; auch die Wasserverfügbarkeit spielt eine eher untergeordnete Rolle bei der Steuerung des fotosynthetischen Flusses, da es zwar zu saisonalen Schwankungen kam, der Regen aber nie vollständig ausblieb.

Schließlich konnten die Flüsse gegen die PPFD-Strahlung ${ }^{13}$ (Photosynthetic Photon Flux Density) dargestellt werden. Die Lichtsättigungsfunktion (Gleichg. 2.28) ließ sich nach der Michaelis-Menten Funktion [HKB ${ }^{+} 94$, LG97, GMR ${ }^{+} 04$ ] entsprechend

$$
F_{c}=\frac{a^{\prime} \cdot P P F D \cdot F_{S a t}}{F_{S a t}+a^{\prime} \cdot P P F D}-R_{T a g}
$$

an die Daten anpassen. Dem Modell folgend, entspricht $\alpha$ der Quantenausbeute ( mol CO$_{2}$ mol absorb. Photonen ${ }^{-1}$ ), $F_{\text {Sat }}$, der Brutto-Fotosyntheserate bei Lichtsättigung ( $\mu \mathrm{mol} \mathrm{m} \mathrm{m}^{-2} \mathrm{~s}^{-1}$ ) und $R_{\text {Tag }}$, der Ökosystematmung am Tage $\left(\mu \mathrm{mol} \mathrm{m} \mathrm{m}^{-2} \mathrm{~s}^{-1}\right)$. Um die Streuung der Daten weitestgehend zu vermindern, wurde der gesamte Datensatz in Lufttemperaturklassen der Breite $2^{\circ} \mathrm{C}$ aufgeteilt. Zusätzlich wurde innerhalb der Temperaturklassen zwischen drei verschiedenen Wolkenbedeckungsgraden unterschieden.

\subsubsection{Untersuchung der saisonalen Variabilität der re- spiratorischen $\mathrm{CO}_{2}$-Flüsse}

Respiratorische Flüsse werden vor allem durch temperaturbedingte Einflüsse gesteuert $\left[\mathrm{VMD}^{+} 00\right]$. Erhöhten Temperaturen folgen erhöhte Veratmungsraten. Beziehung 2.29 beschreibt den exponentiellen Zusammenhang in der

\footnotetext{
${ }^{12}$ Bezeichnet die Summe aus Fotosynthese und Respiration.

${ }^{13}$ Da PPFD-Strahlungsmessungen erst später durchgeführt werden konnten, wurden sie mithilfe der Globalstrahlung entsprechend PPFD $\frac{\mu m o l}{m^{2} s} \approx 2 \frac{\mu m o l}{J} R_{G} \frac{W}{m^{2}}$ angenähert
} 
konventionellen Form in Vielfachen von $Q_{10}$, dem relativen Anstieg der Respiration für einen Temperaturanstieg von $10^{\circ} \mathrm{C}$ :

$$
R_{E}\left(T_{a}\right)=R_{0} Q_{10}^{\left(\frac{T_{a}-T_{m}}{10^{\circ} \mathrm{C}}\right)}
$$

Dabei bezeichnet $R_{0}$ die mittlere nächtliche Ökosystemrespiration, $T_{a}$ die Lufttemperatur und $T_{m}$ die mittlere Lufttemperatur in der Nacht. Etwa $30-80 \%$ der Bruttofotosynthese wird von der Vegetation in Form von Kohlendioxid veratmet und an die Atmosphäre abgegeben. So führt eine hohe Veratmungsrate zu einer geringeren Netto-Produktion von Beständen, was von großer Bedeutung bei der Untersuchung des globalen Kohlenstoffkreislaufs ist.

Die Messung respiratorischer Flüsse in der Nacht stellt eine große Herausforderung dar. Stabile atmosphärische Schichtungsverhältnisse sind mit sehr niedrigen Windgeschwindigkeiten verbunden. Sie führen zu einem stark verminderten turbulenten Austausch - einer Voraussetzung für die Durchführung von Eddy-Korrelationsmessungen. In der Folge der stark unterschätzten $\mathrm{CO}_{2}$-Flüsse ergeben sich fehlerhafte jährliche Summen $\left[\mathrm{BCB}^{+}, \mathrm{GMR}^{+} 04\right]$.

\subsubsection{Das $u_{*}$-Filterkriterium}

Abhilfe kann das sogenannte " $u$-Stern" $\left(u_{*}\right)$ Filter-Kriterium schaffen $\left[\mathrm{GFB}^{+}\right]$. Die Schubspannungsgeschwindigkeit $u_{*}$ dient dabei als Maß für den Turbulenzgrad der atmosphärischen Strömung. Erst nachdem eine gewisse Schwelle $u_{* t h}$ überschritten ist, werden die gemessenen nächtlichen $\mathrm{CO}_{2}$-Flüsse zugelassen. Dabei ist zu beachten, dass mögliche Korrelationen zwischen $u_{*}$ und Temperatur aufgrund täglicher oder saisonaler Zyklen ausgeschaltet werden sollten. Daher ist das Verfahren innerhalb der Jahreszeiten für verschiedene Temperaturklassen durchzuführen. Abbildung 2.9 vermittelt einen Eindruck, wie der $\mathrm{CO}_{2}$-Fluss von der Schubspannungsgeschwindigkeit abhängt. Dargestellt sind exemplarisch zwei aufeinanderfolgende Tage, an denen sich die Werte von $u_{*}$ relativ deutlich unterscheiden.

Um die Ergebnisse der verschiedenen Gruppen auf der Welt vergleichbar zu machen, empfehlen $\left[\mathrm{PRA}^{+} 06\right]$ wie folgt vorzugehen:

- Aufteilung der nächtlichen Daten in 6 Temperaturklassen, wobei jede Klasse die gleiche Anzahl an Daten haben sollte.

- Festlegen der Schwelle $u_{* t h}$, ab der die Maximalflusswerte $99 \%$ ihres Betrages erreicht haben. 

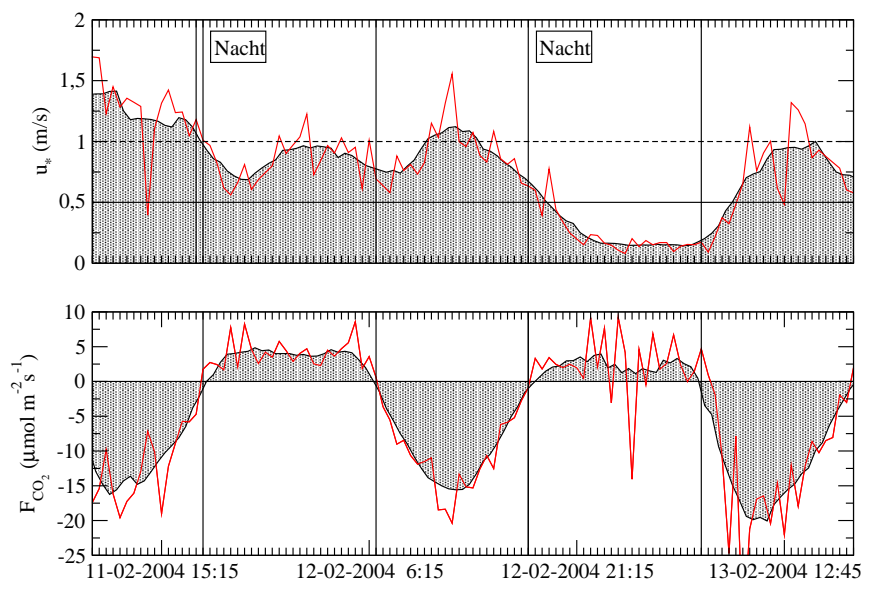

Abbildung 2.9: Darstellung der Schubspannungsgeschwindigkeit $u_{*}$ bzw. des $\mathrm{CO}_{2}$-Flusses zweier aufeinanderfolgender Tage (11.02 bis 13.02. 2004) zur Illustration des Zusammenhanges zwischen Turbulenzgrad und hohen bzw. niedrigen Flusswerten bei Nacht (vereinzelt Datenlücken). Der schattierte Bereich stellt ein gleitendes 3-Stundenmittel dar. Infolge schwach ausgeprägter Turbulenz (niedrige Schubspannungsgeschwindigkeiten bei stabiler atmosphärischer Schichtung) lässt sich der $\mathrm{CO}_{2}$-Fluss in diesen Zeiten nicht adäquat mit der EK-Methode alleine bestimmen.

- Überprüfung, ob die Lufttemperatur der betreffenden Klasse mit $u_{*}$ korreliert. Akzeptanz der Klasse, sofern $|r|<0.4$ gilt.

- Innerhalb der Jahreszeit, wird die Schwelle $u_{* t h}$ als der Median der Einzelschwellen der verschiedenen Klassen festgelegt.

- Für das Jahr wird der maximale saisonale Schwellenwert gewählt.

Bei der Modellierung der respiratorischen Flüsse kam ein speziell auf die Tropen bezogenes Problem hinzu. Die durch Gleichung 2.29 ausgedrückte Beziehung zwischen Temperatur und Respiration ist nur dann praktikabel, wenn die Temperatur während der Nacht über einem hinreichend weiten Temperaturbereich variiert. Am Standort Bariri, jedoch, beträgt die Temperaturspanne über das Jahr hinweg lediglich $5-6^{\circ} \mathrm{C}$, sodass die Flüsse stark streuen und kein klarer Trend erkennbar ist.

Aus diesem Grunde wurde im Rahmen dieser Arbeit ausschließlich auf die Methode der einfachen Mittlung der gefilterten Nachtflussdaten $\left(R_{G}<\right.$ 
Tabelle 2.2: Beziehungen zwischen dem Verhältnis $\left(R_{d f, T} / R_{G, T}\right)$ und der atmosphärischer Durchlässigkeit $\left(R_{G, T} / R_{p o t, T}\right)$. Der tiefgestellte Index $T$ bezeichnet Tageswerte. Ref.: [SHG86]

\begin{tabular}{lrr}
\hline$R_{d f, T} / R_{G, T}=1$ & für & $R_{G, T} / R_{0, T}<0.07$ \\
$R_{d f, T} / R_{G, T}=1-2.3\left(R_{G, T} / R_{0, T}-0.07\right)^{2}$ & für & $0.07 \leq R_{G, T} / R_{0, T}<0.35$ \\
$R_{d f, T} / R_{G, T}=1.33-1.46 R_{G, T} / R_{0, T}$ & für & $0.35 \leq R_{G, T} / R_{0, T}<0.75$ \\
$R_{d f, T} / R_{G, T}=0.23$ & für & $0.75 \leq R_{G, T} / R_{0, T}$ \\
\hline
\end{tabular}

$10 \mathrm{~W} \mathrm{~m}^{-2}$ ) zurückgegriffen. $\mathrm{Zu}$ beachten ist ferner, dass vor Anwendung des Kriteriums, der Speicherterm zu den Flüssen hinzu zu addieren ist, da sonst Gefahr besteht, Flüsse doppelt zu zählen (double counting) $\left[\mathrm{GFB}^{+}\right]$. Zur Bestimmung der Respiration bei Tag $\left(R_{G}>10 \mathrm{~W} \mathrm{~m}^{-2}\right)$ wurden die Erfahrungen aus den Untersuchungen nach den Michaelis-Menten Regressionen ausgenutzt. Dort zeigte sich, dass es zur Bestimmung von $R_{\text {Tag }}$ ausreicht, sich auf den oberen linearen Teil der Licht-Antwort von $F_{b}$ für Globalstrahlungswerte $R_{G}<300 \mathrm{~W} \mathrm{~m}^{-2}$ zu beschränken. Beim Experimentieren mit den Regressionen stellte sich raus, dass Datenfenster von 10 - 20 Tagen ausreichend sind $\left[\mathrm{IRK}^{+}\right]$. Letztlich viel die Entscheidung auf eine Auflösung von zwei Monaten, um die Ergebnisse mit den Resultaten für die nächtliche Respiration vergleichbar zu machen.

\subsubsection{Schließung von Datenlücken (Gap-Filling)}

Nach den kontinuierlichen Messungen in der Zeit vom 15-10-03 bis zum 15-0605 diente mit $98 \%$ ein nahezu vollständiger Datensatz als Grundlage für die nachfolgenden Analysen. Aufgrund von Stationaritätsproblemen sowie dem Ausschluss eines großen Teils der nächtlichen Daten durch die $u_{*}$-Filterung, kommt dem Schließen von Datenlücken zur Bestimmung des NEE eine erhebliche Bedeutung zu.

Bei der Prozedur zur Schließung von Datenlücken wurde zwischen täg- und nächtlichem Datenausfall getrennt. Unabhängig davon wurden Datenlücken $\leq 1.5 h$ linear interpoliert.

Zur Schließung der Datenlücken bei Tag $\left(R_{G}>10 \mathrm{Wm}^{-2}\right)$ wurde wie folgt vorgenommen:

- Saisonale Trennung in Trocken- und Regenzeit.

- Unterteilen der Datensätze in Temperaturklassen.

- Nochmaliges Unterteilen der einzelnen Temperaturklassen in drei ver- 


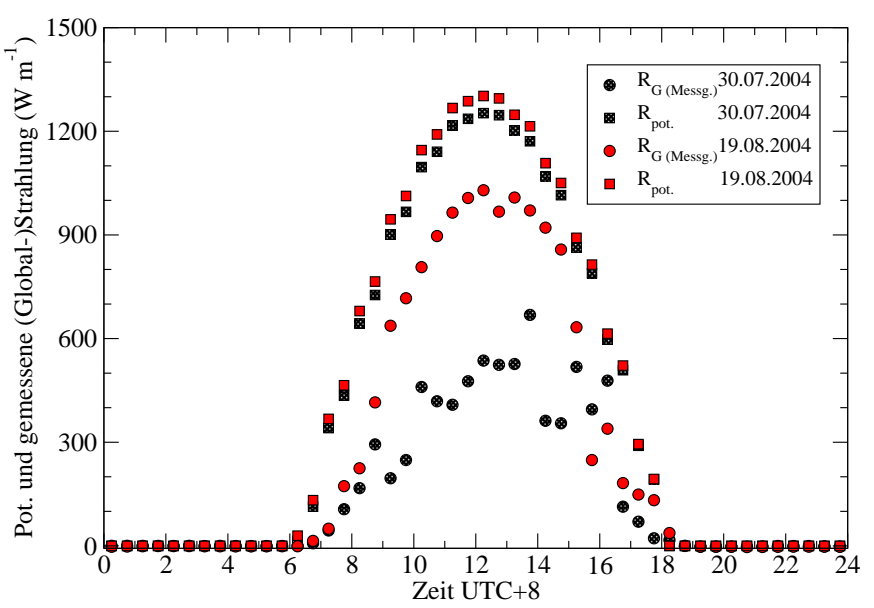

Abbildung 2.10: Zur Bestimmung des Wolkenbedeckungsgrades aus $\left(R_{G} / R_{0}\right)$. Die Grafik zeigt beispielhaft zwei Tage unterschiedlicher Wolkenbedeckung. Während am 30.07.04 klare Wetterverhältisse vorherrschten, weist der 19.08.04 mehr bedeckte Verhältnisse auf (vergl. Tab. 2.2). Die Quadrate bezeichnen $R_{0}$, die Kreise $R_{G}$.

schiedene Wolkenbedeckungsgrade, um zwischen klaren und diffusen Lichtverhältnissen zu trennen.

- Durchführen nichtlinearer Regressionen vom Michaelis-Menten-Typ innerhalb aller Klassen und Unterklassen.

- Füllen der Datenlücken.

Zur Bestimmung des Bedeckungsgrades wurde zunächst die potentielle Sonnenstrahlung (unter Berücksichtigung des Breitengrades, Jahres, Tages im Jahr und der Tageszeit) berechnet:

$$
R_{\text {pot }}=R_{S}\left[1+0.033 \cos 360 t_{T} / 365\right] \sin \beta .
$$

$R_{\text {pot }}$ bezeichnet die potentielle Sonnenstrahlung $\left(\mathrm{J} \mathrm{m}^{-2} \mathrm{~s}^{-1}\right), R_{S}$, die Solarkonstante $\left(1370 \mathrm{~J} \mathrm{~m}^{-2} \mathrm{~s}^{-1}\right.$ ) und $\sin \beta$, den Sinus des Sonnenstandes über dem Horizont; der Cosinus Ausdruck trägt der jährlichen Variation des Abstandes zwischen Erde und Sonne, wobei $t_{T}$ den Tag ab 01.Jan. bezeichnet, Rechnung. So wurde die atmosphärische Durchlässigkeit als Verhältnis von diffuser Strahlung zu Globalstrahlung $\left(R_{d f} / R_{G}\right)$ indirekt aus dem Verhältnis von 
Globalstrahlung zu potentielle Strahlung $\left(R_{G} / R_{\text {pot }}\right)$ abgeleitet (siehe Tab. 2.2). Die Graphik 2.10 veranschaulicht den Verlauf der Globalstrahlung im Verhältnis zur potentiellen Strahlung für zwei unterschiedliche Wolkenbedeckungsgrade (weiteres: siehe Abbildungstext).

Den Ausführungen aus Abschn. 2.2.2 zufolge wurden zur Schließung der nächtlichen Datenlücken die gefilterten Zwei-Monatsmittel verwendet.

Einen profunden Überblick, auch über viele andere Methoden zur Datenlückenschließung (Look-up Tables, neuronale Netzwerke etc.) liefert [FBO $\left.{ }^{+} 01\right]$.

\section{3 Ähnlichkeitstheorie}

\subsubsection{Das Schließungsproblem}

In Abschnitt 2.1.2 wurde beispielhaft anhand der Stoffbilanzgleichung der Übergang von der mittleren Strömung (Gleichg. 2.1) zur turbulenten Strömung (Geichg. 2.2) gezeigt. Dadurch trat ein zusätzlicher Turbulenzterm, der Kovarianzterm $\partial \overline{u_{i}^{\prime} C^{\prime}} / \partial x_{i}$, in Gleichung 2.2 auf. Ähnliche Terme treten auch bei den anderen Grenzschichtgleichungen zur Beschreibung atmosphärischer Turbulenz auf. Die Tatsache, dass das System von Bewegungsgleichungen zur Beschreibung turbulenter Bewegungen mehr unbekannte Parameter als Gleichungen aufweist, wird in der Turbulenzforschung allgemein als Schließungsproblem bezeichnet. Um das Gleichungssystem trotzdem lösen zu können, müssen Annahmen für die zusätzlichen Unbekannten getroffen werden. Eine Möglichkeit zur lokalen Schließung (Schließung 1.ter Ordnung) besteht in dem sog. Mischungsweglängenkonzept [Pra25]. Dieses erfolgt in Analogie zum molekularem Transport (Fick'sches Gesetz) als Proportionalität zwischen einem Fluss und dem Gradienten der entsprechenden Zustandsgröße:

$$
\overline{w^{\prime} \xi^{\prime}}=-K \frac{\partial \bar{\xi}}{\partial z}
$$

mit $K$, dem turbulenten Diffusionskoeffizienten in Richtung der Eigenschaftsdifferenz. Entsprechend der Zustandsgröße $\xi$ wird zwischen turbulenten Diffusionskoeffizienten für Impuls, Wasserdampf, sensible Wärme etc. unterschieden. Aus diesen Betrachtungen heraus lässt sich bereits ein wichtiges Skalierungsgesetz, das logarithmische Geschwindigkeitsgesetz, ableiten. Der turbulente Diffusionskoeffizient für Impuls hat die Dimension (Geschwindigkeit $\times$ Länge) $K_{m} \sim u_{*} l$, wobei $l$, die Mischungsweglänge, gleich $\kappa z$ ist (Prandtl-Hypothese) mit $\kappa$ der Karman-Konstanten. Die relevante 
Geschwindigkeitsskale innerhalb der Bodenschicht ist die Reibungsgeschwindigkeit $u_{*}$. Diese hängt über die Beziehung $u_{*}^{2}=\tau / \rho=-\overline{\left(u^{\prime} w^{\prime}\right)}$ mit der Schubspannung zusammen. Damit ergibt sich aus Gleichung 2.31 für $\xi^{\prime}=u^{\prime}$ nach Integration das logarithmische Geschwindigkeitsgesetz:

$$
u=\frac{u_{*}}{\kappa} \ln z+\text { const. . }
$$

Die Integrationskonstante wird empirisch aus dem Geschwindigkeitsprofil gewonnen. Sie gibt über $\ln z_{0}$, mit $z_{0}$, der Rauhigkeitslänge, Aufschluss über die Oberflächeneigenschaften des überströmten Geländes.

\subsubsection{Monin-Obukhov Ähnlichkeitstheorie}

Die Ausführungen des vorherigen Abschnitts gelten im Grunde genommen nur für neutrale Bedingungen. Mit der 1954 vorgestellten Ähnlichkeitstheorie gelang Monin \& Obukhov [MO58] dann ein Durchbruch. Sie verstanden es, alle Eigenschaften der turbulenten Strömung innerhalb der PrandtlSchicht auf vier relevante Parameter zu reduzieren . Diese sind die Höhe $z$, die Schubspannungs- oder Rauhigkeitsgeschwindigkeit $u_{*}$, der kinematische Wärmefluss $\overline{w^{\prime} T^{\prime}}$ und der Auftriebsparameter $g / T$. Die drei unabhängigen Dimensionen sind Länge, Zeit und Temperatur. Dem Buckingham-П Theorem ${ }^{14}$ folgend ergibt sich daraus genau ein weiterer dimensionsloser Parameter zur Charakterisierung der Vorgänge innerhalb der Prandtl-Schicht. Die nach ihrem Entdecker benannte Obukhovlänge [Stu84]:

$$
L=\frac{u_{*}^{3}}{\kappa(g / T) \overline{w^{\prime} T^{\prime}}} \text {. }
$$

Ihr Betrag lässt sich physikalisch interpretieren als die Höhe einer Luftsäule, in der die Produktion $(L<0)$ respektive der Verbrauch $(L>0)$ kinetischer Turbulenzenergie infolge der Auftriebskräfte gleich der Produktion kinetischer Turbulenzenergie pro Volumenarbeit in einer beliebigen Höhe $z$ innerhalb der dynamischen Unterschicht, multipliziert mal dieser Höhe $z$ ist [Ber95]. Demzufolge stellt $L$ ein Maß für die Stabilität der Schichtung dar. Die drei anderen Skalierungsgrößen sind die bereits erwähnte Schubspannungsgeschwindigkeit, $u_{*}$ und die charakteristische Temperatur, $T_{*}=\overline{-w^{\prime} T^{\prime}} / u_{*}$. Nach der M-O Hypothese werden zahlreiche atmosphärische Parameter, so-

\footnotetext{
${ }^{14}$ Buckingham, Edgar (1867-1940) Das nach Buckingham benannte Theorem ist ein grundlegendes Theorem der Âhnlichkeitstheorie und Dimensionsanalyse.
} 
fern sie geeignet mit $u_{*}$ respektive $T_{*}$ skaliert werden, zu universellen Funktionen von:

$$
\zeta=\frac{z-d}{L}
$$

Der dimensionslose Stabilitätsparameter $\zeta$ ist negativ unter labilen, positiv unter stabilen und nahe Null unter neutralen atmosphärischen Schichtungsbedingungen. Einige wichtige Beispiele universeller Funktionen sind:

$$
\phi_{M}=\frac{\kappa(z-d)}{u_{*}} \frac{\partial \bar{u}}{\partial z} \quad \phi_{H}=\frac{\kappa(z-d)}{T_{*}} \frac{\partial \bar{T}}{\partial z}
$$

wobei $\phi_{M}$ respektive $\phi_{H}$ die dimensionslose Gradientenform von Geschwindigkeit und Temperatur darstellen (ausführlicher z.B. in [Ros03]).

\section{$2.4 \quad 3-D$ Grenzschichtmodellierung}

Die turbulenten Austauschprozesse innerhalb der atmosphärischen Grenzschicht hängen wesentlich von den physikalischen Gegebenheiten der Erdoberfläche ab. Daher erscheint eine umfassendere Untersuchung der Strömungsverhältnisse am Standort Bariri unter Verwendung eines numerischen Grenzschichtmodells, welches sowohl die besonderen Geländeeigenschaften als auch den Einfluss der Vegetationsstruktur einbezieht, als notwendig. Das Strömungsmodell nach Sogachev et al. [SMHL02] erfüllt diese wichtigen Kriterien und bietet darüber hinaus die Möglichkeit zur Bestimmung des sogenannten Footprints [SL04] .

\subsubsection{Theorie des $E-\omega$ Modells}

Das Modell basiert auf den zeitlich und räumlich gemittelten Navier-StokesGleichungen (Reynolds-Gleichungen) mit anderthalbfacher Schließung. Für die Impulserhaltung bei neutralen Strömungsbedingungen gilt dann:

$$
\begin{aligned}
\frac{\partial\left\langle\overline{u_{i}}\right\rangle}{\partial t}+\left\langle\overline{u_{j}}\right\rangle \frac{\partial\left\langle\overline{u_{i}}\right\rangle}{\partial x_{j}}+2 \epsilon_{i j k} \Omega_{j}\left\langle\overline{u_{k}}\right\rangle & =\frac{\partial\langle\bar{P}\rangle}{\partial x_{i}}+\frac{\partial\left\langle\overline{u_{i}^{\prime} u_{j}^{\prime}}\right\rangle}{\partial x_{i}}+S, \\
\frac{\partial\left\langle\overline{u_{i}}\right\rangle}{\partial x_{i}} & =0 .
\end{aligned}
$$


Dabei bezeichnet $P$ den kinematischen Druck und $\epsilon_{i j k} \Omega_{j}$ den Tensor der Erdrotation. Ein Balken über einer Variablen in Verbindung mit eckigen Klammern symbolisiert zeitliche respektive räumlich-horizontale Mittlung. Nach den Ausführungen aus Abschnitt 2.3.1 lassen sich turbulenten Flüsse in der einfachsten Form als Produkt zwischen turbulentem Austauschkoeffizienten und vertikalem Gradienten der entsprechenden mittleren Zustandsgröße ausdrücken (vergl. Gleichg. 2.31). Dem Modell zufolge, werden die turbulenten Schubspannungen entsprechend

$$
\left\langle\overline{u_{i}^{\prime} u_{j}^{\prime}}\right\rangle=\frac{2}{3} \delta_{i j} E-K\left(\frac{\partial\left\langle\overline{u_{i}}\right\rangle}{\partial x_{j}}+\frac{\partial\left\langle\overline{u_{j}}\right\rangle}{\partial x_{i}}\right),
$$

(Boussinesq-Approximation) modelliert [Pie84, RS82]. $K$ ist gleich $C_{\mu} E \omega^{-1}$ und bezeichnet den kinematischen Turbulenz- oder Austauschkoeffizienten (eddy viscosity), mit $E=0.5\left\langle\overline{u_{i}^{\prime} u_{i}^{\prime}}\right\rangle$ der turbulenten kinetischen Energie und $\omega$ der spezifischen Dissipation der turbulenten kinetischen Energie. $\omega$ ist wiederum gleich $\epsilon / E$, mit $\epsilon$ der Dissipationsrate turbulenter kinetischer Energie. $C_{\mu}$ stellt eine Konstante dar und wird weiter unten beschrieben (Glg. 2.50 unten). Mit $S$ in Gleichg. 2.36 werden über die Beziehung

$$
S=-c_{d} A\left\langle\overline{u_{i}}\right\rangle|U|
$$

Verformungs- und viskose Reibungskräfte, hervorgerufen durch die Vegetation, berücksichtigt. Dabei trägt $c_{d}$, der Reibungskoeffizient, der Wechselwirkung zwischen Blattwerk und Bestandesluftströmung Rechnung, und $A$ bezeichnet die Blattfläche pro Einheitsvolumen in $m^{-1}$ oder Blattflächendichte (Leaf Area Density, LAD). Weiterhin gilt: $|U|=\left(\left\langle\overline{u_{i}}\right\rangle\left\langle\overline{u_{i}}\right\rangle\right)^{1 / 2}$. Die Parametrisierung in der verwendeten Form ist bereits in [RS82] veröffentlicht worden. Das primäre Ziel der Modellierungen stellt die Berechnung von Variationen des vertikalen $\mathrm{CO}_{2}$-Flusses in Messhöhe am und um den Turmstandort herum dar. Die Flussvariationen sind Folge von horizontalen respektive vertikalen Strömungsdivergenzen und werden bei neutraler atmosphärischer Schichtung direkt durch das Oberflächenrelief des Geländes hervorgerufen. So lassen sich wichtige Hinweise über die Qualität respektive der Repräsentativität der durchgeführten Flussmessungen am Standort Bariri ableiten. Die Transportgleichungen von $\omega, E$ respektive passiven Skalaren (wie Kohlendioxid und anderer skalarer Spurenstoffe) stellen sich wie folgt dar [Kan04, SRV04]:

$$
\begin{array}{r}
\frac{\partial\langle E\rangle}{\partial t}+\left\langle\overline{u_{j}}\right\rangle \frac{\partial E}{\partial x_{j}}=\frac{\partial}{\partial x_{i}}\left(\frac{K}{\sigma_{E}} \frac{\partial E}{\partial x_{i}}\right)+P_{E}-\varepsilon \\
\frac{\partial \omega}{\partial t}+\left\langle\overline{u_{j}}\right\rangle \frac{\partial \omega}{\partial x_{j}}=\frac{\partial}{\partial x_{i}}\left(\frac{K}{\sigma_{\omega}} \frac{\partial \omega}{\partial x_{i}}\right)+\frac{\omega}{E}\left(C_{1} P_{E}+C_{2}^{*} \omega\right),
\end{array}
$$




$$
\frac{\partial C}{\partial t}+\left\langle\overline{u_{j}}\right\rangle \frac{\partial C}{\partial x_{j}}=\frac{\partial}{\partial x_{i}}\left(\frac{K}{\sigma_{C}} \frac{\partial C}{\partial x_{i}}\right)+S_{C} .
$$

$\sigma_{E}$ ist die Prandtlzahl für turbulente kinetische Energie und $\sigma_{\omega}$ respektive $\sigma_{C}$ sind die Schmidtzahlen für $\omega$ und $C$. Durch

$$
P_{E}=\left\langle\overline{u_{i}^{\prime} u_{j}^{\prime}}\right\rangle \frac{\partial\left\langle\overline{u_{i}}\right\rangle}{\partial x_{j}}
$$

wird die Produktion von TKE, verursacht durch Scherung, berücksichtigt. Um weiterhin den Einfluss der Vegetation in den Gleichungen für $\omega$ und $E$ miteinzubeziehen, bedarf es nach [SP06] keine zusätzlichen Terme in den Gleichungen 2.40 und 2.41. Stattdessen tritt der Koeffizient $C_{2}$, welcher für die Dämpfungsrate der Turbulenz innerhalb der Vegetation steht, in der folgenden modifizierten Form auf (ebd.):

$$
C_{2}^{*}=C_{2}-\frac{\left(C_{2}-C_{1}\right) S_{d}}{E \omega} .
$$

Dabei stellt $C_{1}$ den Koeffizienten für $P_{E}$, dem Produktionsterm aus Gleichg. 2.41, dar. Mit dem Parameter $S_{d}$ wird die verstärkte Dissipation, hervorgerufen durch Reibungseffekte am Blattwerk, berücksichtigt [San03]:

$$
S_{d}=\beta_{d} c_{d} A(z) U E .
$$

Der Parameter $\beta_{d}$ gibt auftretenden Verluste der TKE an; dabei gilt nach [SP06]: $\beta_{d}=12 C_{\mu}^{1 / 2}$. Der Term $S_{C}$ in Gleichg. 2.42 - zur Beschreibung der Quellen und Senken innerhalb der Vegetation - gestaltet sich für neutrale Schichtungsbedingungen in vereinfachter Form.

Die modellierte Quellstärke der Vegetation für $\mathrm{CO}_{2}\left(S_{C}\right)$ [SLK $\left.{ }^{+} 05\right]$ hängt wesentlich von dem aerodynamischen Widerstand $r_{a}$ und dem Stomatawiderstand der Blätter $r_{s}$ ab:

$$
S_{C}=\frac{\alpha A(z)\left(C_{i n}-C\right)}{r_{a}(z)+r_{s}(z)} .
$$

Der Parameter $\alpha$ beschreibt das Verhältnis der totalen zur projizierten Blattfläche. Mit dem Produkt $\alpha A(z)$ lässt sich die totale Blattoberfläche, welche am skalaren Austausch mit der umgebenen Luft teilnimmt, definieren. Mit $C$ wird die $\mathrm{CO}_{2}$ Hintergrundkonzentration und $\mathrm{C}_{i n}$ die $\mathrm{CO}_{2}$ Konzentration in den interzellulären Zwischenräumen bezeichnet. Dabei ist der quasi laminare Widerstand $r_{a}$ wiederum eine Funktion von dem durchschnittl. Blattdurchmesser $D$ und der mittleren Strömungsgeschwindigkeit $U$ [CN98]:

$$
r_{a}=\alpha_{1} \sqrt{\frac{D}{U}}
$$


Tabelle 2.3: Zusammenstellung der verwendeten Modellparameter.

\begin{tabular}{lrr}
\hline Parameter & Wert & Einheit \\
\hline \hline Konstante $\alpha_{1}$ & 240 & $\sqrt{s} / m$ \\
Konstante $\alpha_{2}$ & 500 & $\sqrt{s} / m$ \\
Hintergrundkonzentration C & 380 & $\mathrm{ppm}$ \\
Konzentration (interzell.) $C_{i n}$ & $0.77 \mathrm{C}$ & $\mathrm{ppm}$ \\
Tot./proj. LAI $\alpha$ & 2 & \\
durchschn. Blattdurchmesser $D$ & 0.05 & $\mathrm{~m}$ \\
Sonnenhöhe $h_{s}$ & 45 & $\circ$ \\
\hline
\end{tabular}

Der Stomatawiderstand wird innerhalb der Vegetation als proportional zur Sonnenstrahlung betrachtet [SMHL02]:

$$
r_{s}=\alpha_{2} \exp \left(\frac{0.5 L_{0}(z)}{\sin h_{S}}\right)^{-1},
$$

wobei $L_{0}(z)$ den Blattflächenindex (LAI) und $h_{s}$ die Sonnenhöhe bezeichnet. Die Konstanten $\alpha_{1}$ und $\alpha_{2}$ hängen vom betrachteten Skalar ab; für $C O_{2}$ wurde für $\alpha_{1}=240 \sqrt{s} / \mathrm{m}$ und für $\alpha_{2}=500 \sqrt{s} / \mathrm{m}$ gewählt [CN98]. Für die Hintergrundkonzentration wurde als obere Randbedingung $380 \mathrm{ppm}$ vorgegeben. Diese und weitere Modellparameter sind noch einmal in Tab. 2.3 zusammengestellt. Desweiteren sind Informationen zum Geländerelief von Bedeutung. Informationen zu den Strahlungsbedingungen oder Profilen von Temperatur oder Wasserdampf werden nicht benötigt, da im betrachteten Fall für neutrale Schichtungsverhältnisse gerechnet werden soll.

Das modellierte Untersuchungsgebiet umfasst $2 \times 2 \mathrm{~km}^{2}$, wobei jede Gitterzelle wiederum $50 \times 50 \mathrm{~m}^{2}$ misst, sodass sich ein horizontales Gitter von $41 \times 41=1681$ Punkten ergibt. Die vertikale Schrittweite beträgt im Relief folgenden und im kartesischen Koordinatensystem 3-100 Meter. Die feinere Schrittweiten von 3-10 $m$ dienen zur Auflösung der detailreichen Strömungsprozesse innerhalb der Vegetation und der Prandtl-Schicht.

Angetrieben wurde das Modell mit geostrophischen Windgeschwindigkeiten $U_{g}$ zwischen 8 und $10 \mathrm{~m} \mathrm{~s}^{-1}$ je nach Windrichtung und gemessenen Windgeschwindigkeiten bei neutraler Schichtung. Die turbulenten Längenskalen $l_{0}$ und $l_{\text {max }}$ für die untere und die obere Grenze der atmosphärischen Grenzschicht hängen nach den Beziehungen $l_{0}=k z_{0}$ und $l_{\max }=0.00027 U_{g} / f_{I}$ von der Rauhigkeitslänge $z_{0}$ respektive $U_{g}$ und dem Corolisparameter $f_{I}$ ab ${ }^{15} \cdot l_{0}$

\footnotetext{
${ }^{15}$ Die Bestimmung von $l_{\max }$ war mit Schwierigkeiten verbunden, da der Coriolisparameter für tropische Breitengrade sehr klein ist. Letztlich wurde $l_{\max }$ auf $25 \mathrm{~m}$ festgelegt
} 
und $l_{\max }$ bestimmen wiederum $\omega$ und über die Beziehung $K=C_{\mu} E / \omega$ den kinematischen Turbulenzkoeffizienten.

Aus den obigen Gleichungen verbleiben 5 Koeffizienten, um das Gleichungssystem zu schließen: $C_{\mu}, \sigma_{E}, \sigma_{\omega}, C_{1}$ und $C_{2}$. Lediglich zwei, $C_{1}=0.520$ und $C_{2}=0.833$, erfüllen die theoretischen Betrachtungen abklingender homogener Turbulenz. Experimentell wurden für das Verhältnis $C_{2} / C_{1}$ Werte zwischen 1.6 und 1.7 ermittelt [Pop00, Kan04]. Die anderen Parameter variieren mit den Strömungsbedingungen. Damit legt die Differenz $C_{2}-C_{1}$ das Gleichgewicht zwischen Produktions- und Verlustterm ${ }^{16}$ und damit eine Längeskale fest. Weiterhin sollten die Konstanten die Bedingung

$$
\sigma_{\omega}=\frac{k^{2}}{C_{\mu}^{0.5}\left(C_{2}-C_{1}\right)}
$$

erfüllen, wobei $k \approx 0.43$ die Karman-Konstante bezeichnet. Nur in diesem Fall reproduziert das Modell das logarithmische Geschwindigkeitsgesetz bei neutraler atmosphärischer Schichtung korrekt [Pop00].

Der Einfachheit halber wird das $E-\omega$ Modell ohne Querdiffusionsterm gerechnet, sodass $\sigma_{E}=\sigma_{\omega}$ gilt . Dann lassen sich die turbulente Schmid- und Prandtlzahl durch ihre Abhängigkeit von $C_{\mu}$ bestimmen und $C_{\mu}$ wiederum, lässt sich aus den normalisierten Standardabweichungen ermitteln [KMPS04]:

$$
C_{\mu}=\left[0.5\left(\left(\frac{\sigma_{u}}{u_{*}}\right)^{2}+\left(\frac{\sigma_{v}}{u_{*}}\right)^{2}+\left(\frac{\sigma_{w}}{u_{*}}\right)^{2}\right)\right]^{-2} .
$$

In Ingenieuranwendungen wird $C_{\mu}$ häufig mit 0.09 spezifiziert. Dieser Wert gilt im Wesentlichen für Verhältnisse $P_{E} / \epsilon \approx 1$ und stammt aus Windkanalexperimenten. Die Werte der normalisierten Standardabweichungen hier stammen aus den EK-Messungen, sodass $C_{\mu}$ bei 0.08 liegt [SP06].

Damit ist das System nichtlinearer Differentialgleichungen geschlossen. Zur numerischen Integration wurden die in [SMHL02, SP06] beschriebenen Randund Anfangsbedingungen verwendet. Die Berechnung findet nach der finite Differenzen Methode statt. Für die zeitabhängigen Terme ist eine VorwärtsDifferenzen- und für die advektiven Terme eine Aufstrom-Differenzen Methode implementiert. Die Turbulenzskale $l$ wird nach dem Runge-Kutta Verfahren integriert.

Das Modell wird gerechnet, bis sich ein quasi-stationärer Zustand einstellt.

[Gro93].

${ }^{16}\left(P_{E} / \epsilon \approx 1\right)$ : Bedingung für die Gültigkeit der Beziehung Gleichg. 2.38 (turbulentviscosity hypothesis). 
In der Praxis ist dieser erreicht, wenn das Windfeld, die turbulente kinetische Energie sowie die Matrix der lokalen Austauschkoeffizienten nach fortgeschrittener Rechnung nur noch sehr wenig variieren.

\subsubsection{Strahlungsregime und Vegetation}

Zur Berechnung des Strahlungsregimes wird im Rahmen des Modells zwischen direkter und diffuser Sonnenstrahlung unterschieden. Der tatsächliche spektrale Strahlungsanteil, welcher von der obersten Schicht der Vegetation erreicht wird, hängt von dem astronomischen Sonnenstand, der atmosphärischen Transparenz und von der Wolkenbedeckung ab.

Die Vegetation ist in Form von übereinander angeordneten parallelen Schichten aufgebaut. Diese bestehen aus einem optisch isotropen, trüben Medium. Dabei variiert die Bestandesarchitektur und das Strahlungsregime innerhalb einer Schicht nicht. Wieviel Strahlung vertikal in die Vegetation einzudringen vermag, hängt maßgeblich von der Blattflächendichte (Leaf area density) ab. Eine detaillierte Beschreibung des Strahlungsregimes ist in [SMHL02] zu finden.

\subsubsection{Relief und Koordinatensystem}

Um das Relief miteinzubeziehen, wurde eine digitale Geländekarte (S. Erasmi, Inst. für Geografie, Göttingen) vom Untersuchungsgebiet verwendet. Zur Vermeidung starker Gradienten musste die Topografie geglättet werden. Hierzu wurde ein isotropischer Kosinusfilter (Tukey Filter) verwendet. Dabei wird der neue Reliefpunkt aus seinen Nachbarn wie folgt berechnet:

$$
\begin{gathered}
\overline{f_{i j}}=0.28 f_{i j}+0.13\left(f_{i, j+1}+f_{i+1, j}+f_{i, j-1}+f_{i-1, j}\right)+ \\
0.05\left(f_{i+1, j+1}+f_{i+1, j-1}+f_{i-1, j+1}+f_{i-1, j-1}\right) .
\end{gathered}
$$

$\overline{f_{i j}}$ bezeichnet das geglättete und $f_{i j}$ das ursprüngliche Relief im Gitterpunkt $i, j$ entlang der $x$ respektive der $y$ Achse. An den Gitterrändern wird die Wichtung leicht verändert. Z.B. für den Modellrand im Westen gilt:

$$
\begin{gathered}
\overline{f_{i j}}=0.28 f_{i j}+\frac{0.52}{3}\left(f_{i, j+1}+f_{i, j-1}+f_{i+1, j}\right)+ \\
\frac{0.2}{2}\left(f_{i+1, j+1}+f_{i+1, j-1}\right) .
\end{gathered}
$$

Nach der Glättung sind die wesentlichen Schwierigkeiten, die mit der Berechnung der Ableitung zu tun haben, behoben. Gleichzeitig bleibt der wesentliche Einfluss des Reliefs auf die Strömung erhalten. 
Zur Darstellung und zum Vergleich von Modellergebnissen aus einer bestimmten Höhe wird ein dem Relief anvergl.asstes Koordinatensystem verwendet. Dazu wurde folgende Koordinatentransformation implementiert:

$$
x^{*}=x, y^{*}=y, z^{*}=z-h(x, y),
$$

wobei $x, y, z$ und $x^{*}, y^{*}, z^{*}$ die Koordinaten im kartesischen respektive im modifizierten Gitterbereich bezeichne und $h(x, y)$ die orographische Höhe in Abhängigkeit von $(x, y)$. Ausführlichere Informationen zur Koordinatentransformation liefern z.B. Sogachev et al. [SRV04] und Pielke [Pie84].

\subsection{Footprintanalyse}

Quellgebietsuntersuchungen sind ein wichtiger Bestandteil zur Interpretation von Eddy-Korrelationsmessungen. Sie werden durchgeführt, um festzustellen, welcher Einflussbereich (Footprint) der windzugewandten Seite (Luv) mitsamt den Unterlageneigenschaften vom Sensor erfasst wird (field of view) [Gas86]. Unter dem Footprint am Ort des Messpunktes wird nach der gängigen Definition in [Fok03] der mit der Footprint-Funktion $\phi$ gewichtete Einfluss der Eigenschaften $Q$ (Quellen) an der Luvseite ${ }^{17}$ des Messgerätes verstanden. Er lässt sich wie folgt in integraler Form darstellen [SLMD90]:

$$
\eta\left(x_{m}, y_{m}, z_{m}\right)=\int_{-\infty}^{\infty} \int_{-\infty}^{\infty} Q_{\eta}\left(x^{\prime}, y^{\prime}, z^{\prime}\right) \phi\left(x_{m}-x^{\prime}, y_{m}-y^{\prime}, z_{m}-z_{0}\right) d x^{\prime} d y^{\prime}
$$

wobei $z=z_{0}$ das Oberflächenniveau des Quellgebiets und $\left(x_{m}, y_{m}, z_{m}\right)$ den Messpunkt bezeichnet.

In der Vergangenheit wurden viele verschiedene Ansätze entwickelt, um die Footprint-Funktion abzuschätzen. Dazu sind im Wesentlichen drei Klassen von Modellen zu nennen: Analytische, Lagrange stochastische (LS) und Modelle, die auf der Lösung der Navier-Stokes Gleichung (NSG) basieren (eine Übersicht liefern Schmid et al.[Sch02]).

\footnotetext{
${ }^{17}$ Gerade über komplexem Gelände haben unlängst Untersuchungen gezeigt, dass auch die windabgewandte Seite durch rückwärtsgewandte Strömungen (reverse flow) zum Fluss beitragen kann [SRV04].
} 


\subsubsection{Prinzip des NSG-Modells nach Sogachev et al.}

Jüngere Untersuchungen befassen sich vor allem mit störenden Einflüssen auf die Berechnung das Quellgebietes, wie sie durch heterogene oder diskontinuierliche Vegetationsschichten verursacht werden [GRF03, $\mathrm{RGF}^{+} 05$, RAK00]. Es existieren bis heute aber nur sehr wenige Untersuchungen, die den Einfluss eines komplexen Geländereliefs mit einbeziehen. Das hier verwendete Modell von Sogachev et al. [SRV04, SMHL02] bietet diese Möglichkeit und soll demzufolge noch einmal unter diesem Aspekt näher beleuchtet werden. Ein wesentliches Merkmal des Modells ist, dass die turbulenten Flüsse durch Fluss-Gradient Beziehungen (vergl. Abschn. 2.3.1) berechnet werden. Die Anwendbarkeit dieser Beziehung innerhalb der Vegetation wird in Sogachev et al. [SL04] kontrovers diskutiert. Gross [Gro93] folgend, ist dieser Ansatz insbesondere in 2D- und 3D-Modellierungen vertretbar, wenn der advektive Transport von größerer Relevanz ist als der diffusive Transport. Dies wurde sowohl von Sogachev et al. [SMHL02] selber als auch von Gross [Gro87] durch zahlreiche erfolgreiche Modellrechnungen bestätigt. Mithin darf der Ansatz als validiert gelten und wird somit für diese Rechnungen verwendet.

Das Prinzip der Methode zur Footprint-Bestimmung basiert auf der Schätzung des Beitrags jeder einzelnen Gitterzelle zum vertikalen Skalarfluss am Ort des Interesses - also in der Regel am Ort des Messgerätes. Die einzelnen Zellen des Gitters werden der Reihe nach aktiviert, sodass die einzelnen Beiträge der Quellen (oder Senken) zum vertikalen Fluss berechnet werden können. Anschließend werden die einzelnen Flussbeiträge superponiert und danach normalisiert. Das Ergebnis ist eine Quell-Gewichts- oder Footprintfunktion, die den Beitrag einer jeden Zelle zum Fluss angibt. Dabei ist anzumerken, dass die berechnete Footprint-Funktion streng genommen nicht $\phi$ aus Gleichung 2.54 entspricht. Jene hängt lediglich von der turbulenten Diffusion und von der Quelle-Sensor-Entfernung ab. Tatsächlich handelt es sich um eine normalisierte Beitragsfunktion, bei der eine horizontal variierende Flussverteilung per Definition Variationen in $\phi(\bar{r})$ nach sich zieht. Für den speziellen Fall, dass die Flüsse horizontal homogen verteilt sind, lässt sich $\phi$ aber als äquivalent betrachten.

Zur Modellierung der vertikalen Verteilung der $\mathrm{CO}_{2}$-Senkenstärke innerhalb der Vegetation wurde die am Turmstandort gemessenen Blattflächendichteverteilung verwendet [Die07]. Dazu wurde der Kronenraum vertikal in zwei Meter dicke Schichten unterteilt und mit einer Senkenstärke proportional zur $L A D$ versehen. Ebenso wurde auch die Bodenrespiration den Messungen entnommen. Damit erscheint es möglich sowohl den Nettofluss, d.h. den respiratorischen und den assimilatorischen Fluss insgesamt, als auch den Ein- 


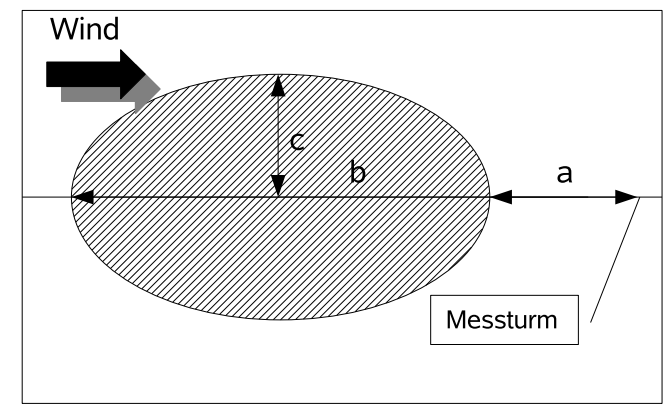

Abbildung 2.11: Bestimmung des Quellgebiets der Hauptwindrichtungen nach dem analytischen Modell von Kormann-Meixner. Die Ellipsenparameter $a, b$ und $c$ entsprechen dem Mittelwert des auf Halbstundenbasis berechneten Quellgebiets aus dem 90\% des Flusses stammt. Ref::[KM01].

fluss von Quellen und Senken separat für den Ort des Sensors zu rechnen. Im Unterschied zum Fall über horizontalem Gelände sind negative Werte der Quell-Beitragsfunktion prinzipiell nicht mehr ausgeschlossen. So würde ein negativer Beitrag die Reduzierung des Signals an dieser Stelle bedeuten.

\subsubsection{Das analytische Modell nach Kormann-Meixner}

Neben den numerischen Untersuchungen wurde zum einen zu Vergleichszwecken und zum anderen um Hinweise über Flussbeiträge, wie sie insbesondere bei stabiler atmosphärischer Schichtung zu erwarten sind, zu bekommen, das analytische Footprint-Modell nach Korman-Meixner (KM) gerechnet [KM01]. Ein Vorteil des KM-Modells besteht in der Möglichkeit, verschiedene atmosphärische Schichtungen zu betrachten.

$\mathrm{Zu}$ den wesentlichen Merkmalen des KM-Ansatzes zählt, dass die vertikalen Profile der Windgeschwindigkeit und des Austauschkoeffizienten (eddy diffusivity) nach den Potenz-Gesetz Ansätzen

$$
\begin{aligned}
& u(z)=U z^{m}, \\
& k(z)=K z^{n}
\end{aligned}
$$

parametrisiert werden. Dabei sind die Ansätze indirekt über die Koeffizienten $m$ und $n$ mit der Monin-Obukhov Theorie (vergl. Abschn. 2.3.2) verbunden ${ }^{18}$. Quer zur Windrichtung wird die Footprint-Funktion durch eine

\footnotetext{
${ }^{18}$ Für die Koeffizienten gilt: $m=f\left(u_{*}, L, z_{r}, z_{0}\right)$ und $n=f\left(u_{*}, L, z_{r}, z_{0}\right)$.
} 
Tabelle 2.4: Parameter zur Charakterisierung des Rauhigkeitseigenschaften des Regenwaldbestandes am Standort Bariri. Ref:: [IRK $\left.{ }^{+}\right]$

\begin{tabular}{|c|c|}
\hline Parameter & $(\mathrm{m})$ \\
\hline Rauhigkeit $z_{0}$ & 2.7 \\
\hline Versatzhöhe $d$ & 27.3 \\
\hline Messhöhe $z_{r}$ & 48.0 \\
\hline Bestandeshöhe(max.) $z_{1}$ & 33.0 \\
\hline
\end{tabular}

Gauss-Verteilung beschrieben:

$$
Q(x, y)=\frac{1}{\sqrt{2 \pi \sigma}} \exp \left(-\frac{y^{2}}{2 \sigma^{2}}\right)
$$

wobei $\sigma_{v}$, die Standardabweichung der Querwindfluktuation, $u_{*}$ die Schubspannungsgeschwindigkeit, $L$ die Monin-Obukhov Länge, dir die Windrichtung sowie $z_{r}$ die Mess- und $d$ die Versatzhöhe als Eingabevariable für das KM-Modell dienen. Letztlich ergibt sich die Footprintfunktion zu:

$$
\phi(x, y)=Q(x, y) A \exp \left(-\frac{B}{x}\right) x^{C}
$$

Dabei wird $\phi$ über die meteorologischen Eingabevariablen durch fünf Parameter festgelegt. Neben $A, B, C$ aus Gleichg. 2.58, kommen zwei weitere, $D$ und $E$, aus der Parametrisierung von $\sigma\left(\sigma=D x^{E}\right)$ in Gleichg. 2.57 hinzu. Eine detailliertere Beschreibung ist in [KM01] zu finden.

Die Analyse für die Messungen am Turmstandort wurde für die Halbstundenstatistik des gesamten Datensatzes der Hauptwindrichtungsbereiche $\left(80^{\circ}-100^{\circ}\right),\left(230^{\circ}-250^{\circ}\right)$ und $\left(290^{\circ}-310^{\circ}\right)$ duchgeführt. Anschließend wurden für jede Halbstundenstatistik das Quellgebiet (source area), welches den prozentualen Beitrag zum Fluss markiert, berechnet. Dazu wurde das Quellgebiet auf die relevanten Maßstäbe (Länge, Breite und Abstand vom Messturm), sprich, auf die drei Ellipsenparameter $a, b$ und $c$ reduziert (vergl. Abb. 2.11 ). Das Quellgebiet ergibt sich schließlich durch einfache Mittlung der berechneten Parameter, wobei nach atmosphärischer Schichtung und Windrichtung zu klassifizieren ist. Die verwendeten standortspezifischen Parameter sind in Tabelle 2.4 aufgeführt. 


\subsubsection{Zusammenfassung}

Der wesentliche Vorteil des NSG-Footprintmodells [SL04] gegenüber den analytischen [HW92, GRF03] und den LS-Footprintmodellen besteht in der Berücksichtigung des komplexen Strömungsregimes als Folge des inhomogenen Geländereliefs. Die Topografie beeinflusst die Plume-Dispersion zum einen durch eine veränderte Hauptströmung (was den Weg, welchen der Plume nimmt, beeinträchtigt) und zum anderen durch Turbulenz (was die Rate der dispersiven Verbreiterung des Plumes quer zur Windrichtung erhöht) [SRV04]. Zudem kann es in Abhängigkeit von der Topografiebeschaffenheit zu Strömungsablösungen kommen [RWCH92].

Das Modell wird in dieser Phase der Entwicklung ausschließlich für neutrale Schichtungsverhältnisse verwendet. Mit dieser Untersuchung wird - nach ermessen des Autors - erstmals der Versuch unternommen, den störenden Einfluss komplexer Geländebedingungen in 3 Dimensionen zu untersuchen. 


\section{Kapitel 3}

\section{Ergebnisse}

\subsection{Meteorologie und Turbulenz am Standort}

Neben den eigentlichen turbulenten Flussmessungen wurden eine Reihe weiterer meteorologischer Messungen durchgeführt. Die meteorologischen Messungen sind zur Charakterisierung des Standorts unbedingt notwendig; insbesondere das lokale Windsystem liefert bei einem Standort mit komplexer Orografie wichtige Hinweise für die EK-Messungen. Aber auch die Messungen von Globalstrahlung respektive fotosynthetisch aktiver Strahlung sind beispielsweise für die Untersuchungen zur Energiebilanzschließung obligatorisch.

\subsubsection{Das lokale Windsystem}

Das lokale Windsystem am Standort Bariri zeichnet sich durch eine bemerkenswerte Stationarität aus. Über den gesamten Messzeitraum (15.10.200315.06.2005), so zeigten die auf Monatsbasis durchgeführten Untersuchungen (nicht gezeigt), änderte sich die Form der Windrose (Abb. 3.1) quasi nicht. Schwächere Winde $<3 \mathrm{~m} / \mathrm{s}$ durchlaufen einen das Jahr hindurch charakteristischen Tagesgang. Ausgangspunkt sind durch nächtliche Ausstrahlung (radiative cooling) hervorgerufene Schwereströmungen (katabatic or drainage flows) [SK88, SIB02, MVN01, AHY03]. Kühle und deswegen vergleichsweise dichtere Luftmassen fließen aufgrund der Gravitation hangabwärts. Über $80 \%$ und damit der weit überwiegende Anteil aller nächtlichen Winde $<3 \mathrm{~m} / \mathrm{s}$ entstammen den Bergregionen südwestlich des Turmes. Nicht nur die stets 


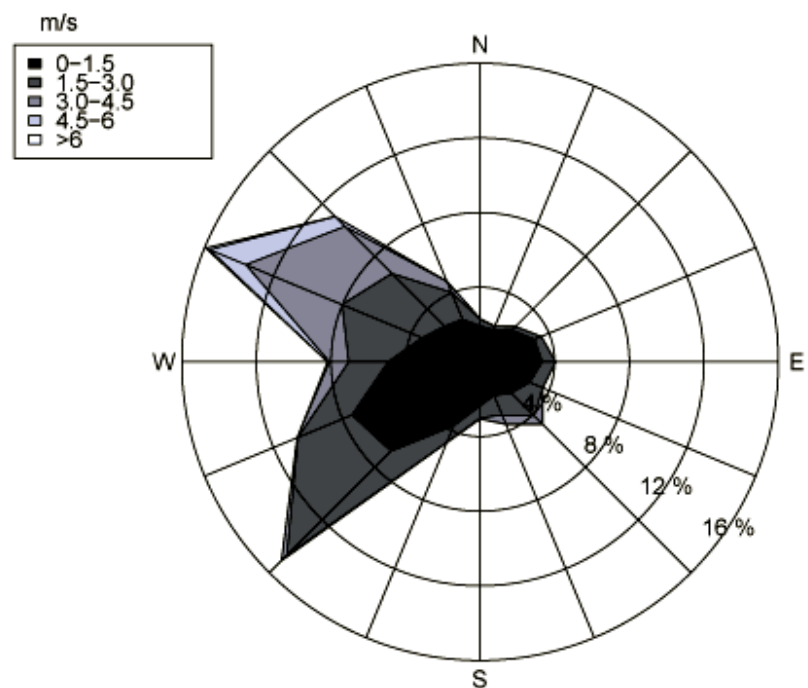

Abbildung 3.1: Windrose des Jahres 2004. Dargestellt ist die prozentuale Häufigkeit der einzelnen Windklassen. Die Messungen aus 48m Höhe wurden mit dem Ultraschallanemometer (USA-1, METEK) durchgeführt.

hangabwärts gerichtete Windrichtung, auch die Ergebnisse aus den Temperaturprofilmessungen (nicht gezeigt), liefern starke Hinweise darauf, dass es sich um katabatische Schwereströmungen handelt. Unter solchen Bedingungen ist eine Entkopplung der unteren Luftschichten wahrscheinlich. Insbesondere bei Wäldern kann die Vegetation kanalisierende Wirkung haben, sodass die katabatische Strömung auf den Stammraum begrenzt wird [AHY03]. Am Morgen dann, gleich nach Sonnenaufgang (7.00h) beginnt die Windrichtung innerhalb der nächsten Stunde über Süd zu drehen (vergl. Abb. 3.2 a), um bis kurz vor Mittag aus östlichen Richtungen zu wehen. Anschließend dreht der Wind erneut, aber langsamer, gegen den Uhrzeigersinn, um schließlich zum Sonnenuntergang (18.00h) wieder aus südwestlichen Richtungen zu wehen. Stärkere Winde $(>3 \mathrm{~m} / \mathrm{s})$ wehen beinahe ausschließlich aus WNW-lichen Richtungen (vergl. Abb. 3.1); außerdem sind sie tagsüber etwa 4 mal häufiger als in der Nacht.

Einen detaillierteren Einblick in die lokale Strömungsdynamik liefert die Abbildung 3.2 (b). Dargestellt ist die Schubspannungsgeschwindigkeit $u_{*}$, der Stabilitätsparameter $\zeta$ und die gemittelte Vertikal- respektive Horizontal- 

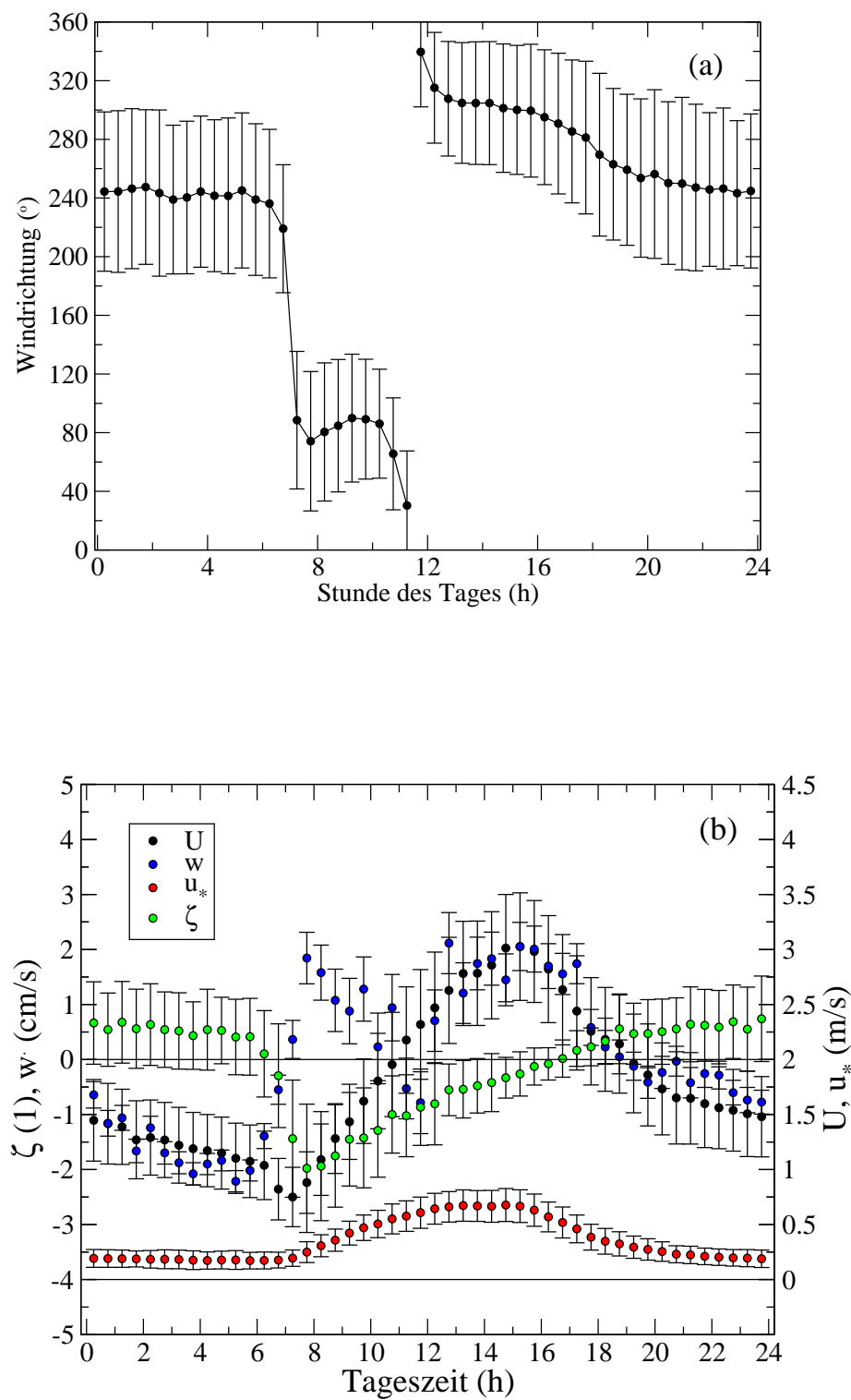

Abbildung 3.2: Dargestellt ist der Tagesgang (a) der mittleren Windrichtung für alle Windgeschwindigkeiten respektive (b) der Tagesgang der gemittelten Horizontal- $(U)$ und Vertikalwindgeschwindigkeit $(w)$ sowie des Stabilitätsparameters $(\zeta)$ und der Schubspannungsgeschwindigkeit $\left(u_{*}\right)$ des Jahres 2004. 
windgeschwindigkeit in Abhängigkeit von der Tageszeit. Dabei ist die Vertikalwindgeschwindigkeit als Indikator für mögliche advektive Flüsse in vertikaler Richtung von Bedeutung. Die Abbildung zeigt sehr anschaulich, wie im Verlauf der Nacht $(\zeta>0)$, die in den Bestand gerichtete Vertikalwindgeschwindigkeit mit abnehmender Horizontalwindgeschwindigkeit, kontinuierlich zunimmt. Sofort nach Sonnenaufgang (6a.m. - 7a.m.) schlägt die Stabilität $(\zeta<0)$ und mithin die Vertikalwindgeschwindigkeit $(w>0)$ um. Gleichzeitig nimmt die Turbulenz innerhalb des Strömungsregimes langsam $\mathrm{zu}\left(u_{*}>0.3 \mathrm{~m} \mathrm{~s}^{-1}\right)$. Generell zeigen die Untersuchungen, dass tagsüber positive (aus dem Bestand heraus gerichtete) und nachts negative (in den Bestand hinein gerichtete) advektive Strömungen vorherrschen. Durch die stabilen nächtlichen Schichtungsverhältnisse $(\zeta>0.5)$ verbunden mit niedrigen Windgeschwindigkeiten $\left(U<2 \mathrm{~m} \mathrm{~s}^{-1}\right)$, erreicht $u_{*}$ als Maß für Turbulenz im Mittel - über die Nacht - selten Werte über 0.20 oder $0.25 \mathrm{~m} \mathrm{~s}^{-1}$. Dadurch ist der vertikale turbulente Austausch, der für EK-Messungen vorauszusetzen ist, stark eingeschränkt. Abhilfe kann - so die Annahme - das bereits eingeführte $u_{*}$-Kriterium (vergl. Abschn. 2.2.2) schaffen. An diesem Punkt stellt sich bereits die entscheidende Frage zur Berechnung des NEE: Nämlich, ob und wenn ja, in welchen Ausmaß advektive $\mathrm{CO}_{2}$-Flüsse auftreten? Inwieweit haben diese Strömungen das Potential, die Ergebnisse aus den turbulenten Flussmessungen zu ergänzen. Eine quantitative Abschätzung dazu folgt in Abschnitt 3.4.1.

\subsubsection{Meteorologische Eigenschaften}

Um den Messstandort Bariri meteorologisch charakterisieren zu können, werden in Abb. 3.3 die relevantesten meteorologischen Parameter angegeben. Dargestellt sind von oben nach unten die Globalstrahlung $\left(R_{G}\right)$, die Temperatur $(T)$, das Wasserdampfdefizit $(V P D)$ und der Niederschlag $\left(N_{d}\right)$ im Tagesmittel. Mit Ausnahme des Niederschlages, welcher von einer 1-2km entfernten Meteorologiestation stammt, wurden die Werte in $48 m$ Höhe $(15 m$ über dem Vegetationsbestand) gemessen.

Die jährliche Durchschnittstemperatur liegt bei $19.1^{\circ} \mathrm{C}$. Die Variation der Temperatur im Verlauf des Jahres ist mit $\pm 2.5^{\circ} \mathrm{C}$ äußerst gering. Das Wasserdampfdefizit zeigt in den Monaten Aug-Sep. 2004 zwei interessante Peaks, welche sich etwas weniger ausgeprägt auch bei der Globalstrahlung wiederfinden lassen. Offensichtlich lagen in diesem Zeitraum besonders klare Wetterbedingungen vor. Darauf deutet auch der leichte Temperaturabfall, welcher mit forcierter nächtlicher Ausstrahlung erklärt werden kann und die niedrige Niederschlagsmenge von im Mittel unter $4 \mathrm{~mm}$ pro Tag, hin. 
Saisonaler Verlauf der wichtigsten meteorologischen Parameter

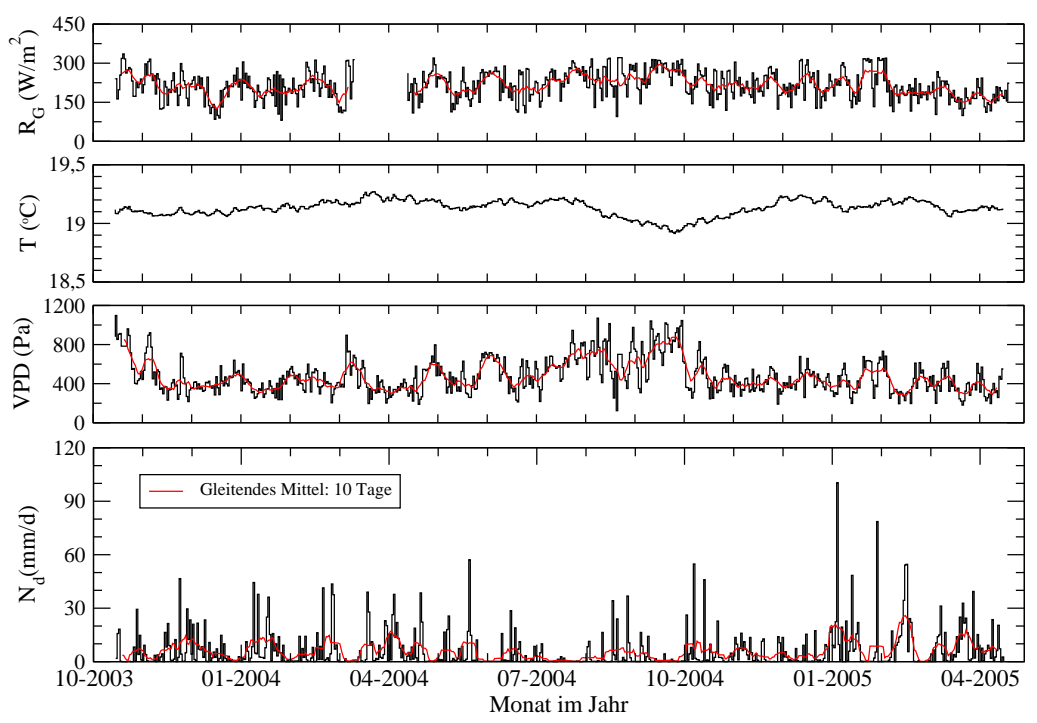

Abbildung 3.3: Der saisonale Gang der relevantesten meteorologischen Variablen für die Messperiode (10/2003 - 06/2005). Dargestellt sind Globalstrahlung $\left(R_{G}\right)$, Lufttemperatur $(T)$, Wasserdampfdefizit $(V P D)$ und der Niederschlag $\left(N_{d}\right)$ aus $48 m$ Höhe im Tagesmittel. Zusätzlich wurde (rot) ein gleitendes 10-Tagesmittel auf die Daten angewandt.

Die Messperiode lässt sich unterteilen in eine etwas regnerische erste Jahreshälfte $(6.6 \mathrm{~mm} / \mathrm{Tag})$ und eine weniger regnerische zweite Jahreshälfte (3.9mm/Tag) (vergl. auch Abb. 3.4). Die regnerische und weniger regnerische Periode zusammengenommen ergeben insgesamt 1641 $\mathrm{mm}$ Niederschlag für das Jahr 2004.

\subsection{Bowen-Verhältnis}

Das Bowen-Verhältnis (Bowen-Ratio) gibt das Verhältnis fühlbarer Wärmestrom $H$ zu latentem Wärmestrom $\lambda E$ an.

Abgesehen von den in Abschnitt 3.1 .2 bereits diskutierten Monaten mit hohem VPD Aug-Sep. 2004 übersteigt der latente Wärmestrom den fühlbaren Wärmestrom deutlich. Mit mittleren Bowen-Verhältnis Werten von $0.47 \pm 0.17$ für die regnerische und $0.56 \pm 0.17$ für die weniger regnerische Saison werden die entsprechenden Niederschlagsmengen aus Abschnitt 3.1.2 


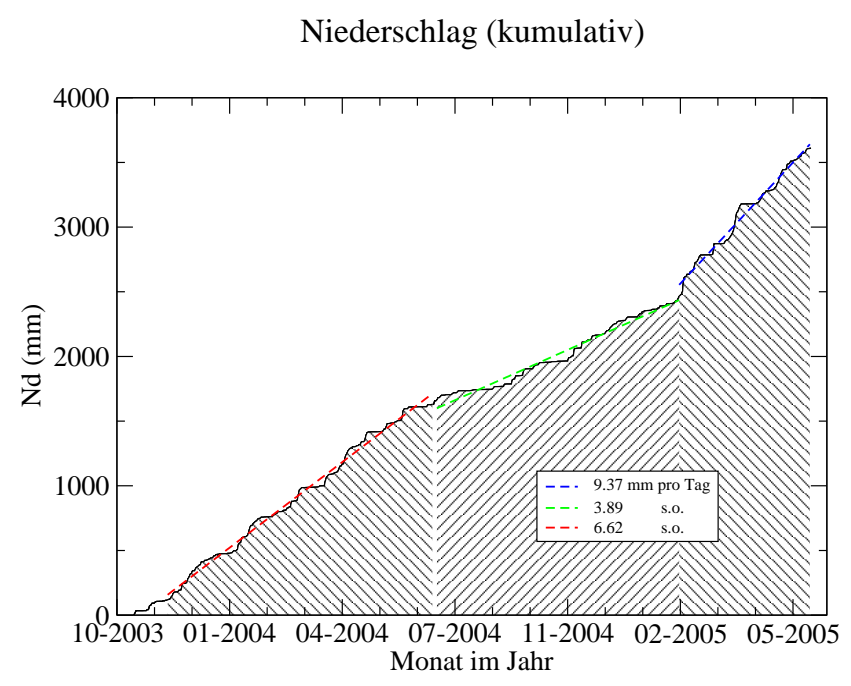

Abbildung 3.4: Kumulativ dargestellter Niederschlag für die gesamte Messperiode. Deutlich zu erkennen: die regnerische $(6.6 \mathrm{~mm} / \mathrm{Tag})$ und weniger regnerische $(3.9 \mathrm{~mm} / \mathrm{Tag})$ Jahreshälfte 2004.

plausibel wiedergespiegelt.

\subsection{Methoden zur Sicherung der Datenqualität}

\subsubsection{Der Turmeffekt: Beeinträchtigung der Datenqua- lität durch den Turm}

Eine besonders krude Beeinträchtigung der Datenqualität findet statt, wenn der Messturm (Abb. 2.6) die Flussmessungen stört. In der Regel wird empfohlen, den gestörten Bereich weiträumig (direkte Abschattung $\pm 30^{\circ}$ ) auszuschließen [KF94]. Da bei diesem Verfahren u.U. ein Großteil aller verfügbaren Daten einer Messkampagne verloren geht, wurde 1997 am Inst. für Bioklimatologie eine sehr einfache Methode entwickelt, um die verfälschten Daten zu korrigieren [Fal97, Ibr01, $\mathrm{IST}^{+} 96$ ].

Indem der Wind Gerüsttürme der Bauart Abb. 2.6 passiert, werden die vertikalen Fluktuationen, ausgedrückt durch die Standardabweichung der Vertikalfluktuationen $\sigma_{w}$, in Abhängigkeit vom Abstand zwischen Turm und 


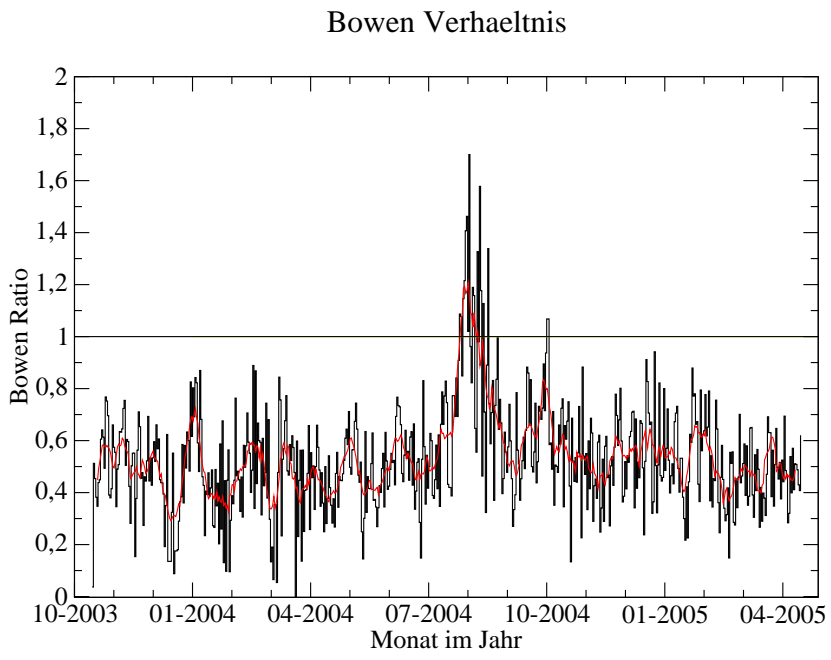

Abbildung 3.5: Bowen Ratio (Verhältnis von sensiblem zu latentem Wärmestrom): Dargestellt ist das Tagesmittel für $R_{G}>50 \mathrm{~W} \mathrm{~m}^{-2}$. Zusätzlich wurde (rot) ein gleitendes 10-Tagesmittel auf die Daten angewandt.

Messgerät gedämpft. Gleichzeitig werden die Horizontalfluktuationen intensiviert, sodass $u_{*}$ als Kovarianz von $u$ und $w$, keine turmabhängige Windrichtungsabhängigkeit aufweist [Ibr01]. Dies wurde überprüft, indem der nach SSO weisende Ausleger des stationären Systems entgegengesetzt in Richtung NNW verlängert wurde. Dort wurde ein zweites EK-Messsystem in etwa $2.5 m$ Abstand vom Turm installiert ${ }^{1}$.

Abbildung 3.6 (a) zeigt den Einfluss des Turmes ausgedrückt durch das Verhältnis $\sigma_{w(S S O)} / \sigma_{w(N N W)}$ sehr deutlich. Eine direkte Abschattung - verursacht durch die äußersten Gerüstteile - besteht im Bereich zwischen 323.2 ${ }^{\circ}$ und $341.1^{\circ}$ (vergl. Abb. 2.7 b). Verglichen, mit Wind aus SSO $\left(\approx 150^{\circ}\right)$, bedeutet Wind aus NNW-lichen Richtungen nur einen sehr kleinen Effekt, da durch den großen Abstand zum Turm (4.5m im Lee) die Dämpfung wesentlich kleiner ausfällt. Damit wird auch deutlich, dass sich bei der Verwendung massiver Gerüsttürme, die Qualität von Flussmessungen effektiv verbessern lässt, wenn neben der Hauptwindrichtung auch besonders die Auslegerlänge Berücksichtigung findet.

Zur Korrektur wurde die systematisch von der Windrichtung abhängende

\footnotetext{
${ }^{1}$ Der zweite Ausleger bestand aus einem weniger massiven Rohr, sodass ein Abstand von $4.5 \mathrm{~m}$ zwischen Turm und Messsystem aus Stabilitätsgründen nicht zu erreichen war. Von der Montage eines zweiten massiven $4.5 \mathrm{~m}$ Auslegers musste schon allein aus turmstatischen Gründen abgesehen werden.
} 
Abweichung von der Vertikalwindfluktuation mithilfe der Funktion:

$$
\frac{\sigma_{w}(S S O)}{\sigma_{w}(N N W)}=1-\frac{1}{a \sqrt{\pi}} \cdot \exp \left(-b \cdot \frac{(d i r-c)^{2}}{a^{2}}\right)
$$

angepasst, wobei sich die drei Parameter nach Iteration $\mathrm{zu} a=10.61, b=$ 0.10 und $c=330^{\circ}$ bestimmen ließen und dir die Variable für die Windrichtung bezeichnet. Die Korrekturfunktion respektive die Korrektur nach Falk (1997) ergibt sich schließlich zu $f_{\text {korr }}=2-$ Fit respektive $w_{\text {korr }}^{\prime}=$ $f_{\text {korr }}($ dir $) \cdot w^{\prime}$.

Letztendlich ist der Einfluss auf die Flüsse (beim $\mathrm{CO}_{2}$-Fluss beträgt er 1.33\%) aus zwei Gründen verhältnismäßig klein. Zum einen ist die Windrichtung zumindest im Kernbereich der Korrektur $\left(323.2^{\circ}-341.1^{\circ}\right)$ seltener (vergl. Abb. $3.6 \mathrm{~b}$ ) und zum anderen betragen die Korrekturfaktoren aus $f_{\text {korr }}$ kaum Werte über 1.05. Verglichen mit anderen Standorten (vergl. z.B. [Fal97]) sind die durchgeführten Korrekturen vernachlässigbar klein.

\subsubsection{Die Footprintanalyse}

Das verwendete Modell nach Sogachev et al. (2002) stellt eines der wenigen Modelle dar, mit dem Strömungsbeeinflussungen, wie sie durch ein heterogenes Geländerelief hervorgerufen werden, berücksichtigt werden können. Zudem wurde der Höhenabhängigkeit der $\mathrm{CO}_{2}$ Senkenstärke innerhalb der Vegetation Rechnung getragen, indem das $L A D$ Profil vom Standort Bariri zur Modellierung der Flüsse verwendet wurde. Der mittlere respiratorische Fluss am Boden stammt aus Quellgebietsuntersuchungen vor Ort [Jin06]. Dargestellt sind jeweils die Quellgebiete der drei Hauptwindrichtungen, aus denen 50,70 und $90 \%$ der $\mathrm{CO}_{2}$-Flüsse stammen [Sch02]. Die Abbildungen 3.7 (a,c und e) zeigen jeweils den Einflussbereich, wie er über einem homogen flachem Geländerelief zu erwarten ist ${ }^{2}$. Zum Vergleich wurde die gleiche Quellgebietsanalyse mit dem KM-Modell wiederholt (siehe Abb. 3.8). Da das KM-Modell lediglich Quellen einer vorgegebenen Höhe berücksichtigt, wurde das Quellgebiet in einer Höhe lokalisiert, die der Versatzhöhe $d$ plus der Rauhigkeitslänge $z_{0}$ entspricht. Erwartungsgemäß stimmen beide Modelle für die betrachteten neutralen Schichtungsverhältnisse gut überein [SL04]. Dagegen ist auf den Abbildungen 3.7 b,d und f deutlich der Einfluss der Topografie ersichtlich. Zunächst einmal stellt sich heraus, dass sich der Footprint-Bereich bei Wind aus östlicher Richtung (d) am stärksten verändert hat. Jedoch tritt

\footnotetext{
${ }^{2}$ Die vorzugebenen mittleren Windgeschwindigkeiten $U$ sowie $z_{r}, z_{0}, d$ etc. wurden den Messungen entnommen.
} 
Zur Daempfung der Vertikalwindfluktuationen durch den Turm

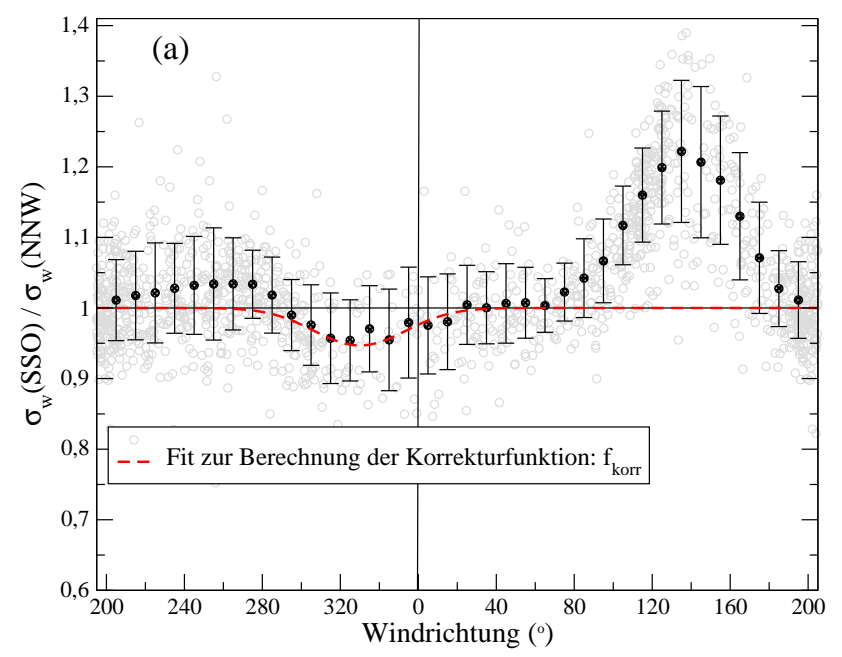

Relative Haeufigkeit der Observationen in Abh. v.d. Windrichtung

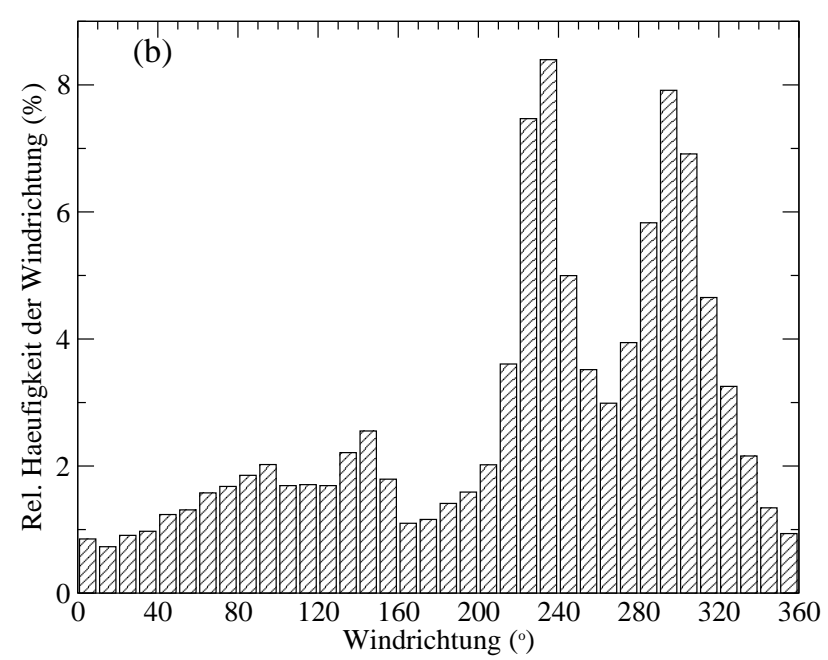

Abbildung 3.6: Dargestellt ist (a) die Funktion $f_{\text {korr }}$ zur Korrektur der turmbedingten Dämpfung der Vertikalwindfluktuationen $\left(\sigma_{w}\right)$ und (b) die relative Häufigkeit der Vertikalwindfluktuationen in Abhängigkeit von der Windrichtung für das stationäre Ultraschallanamometer (USA-1). 
die Modifikation erst auf, nachdem 90\% des Quellgebietes (vergl. Abb. 3.7 c) einbezogen wurden. Wahrscheinlich ist die nach Osten verlaufende Anhöhe des Turmstandortes $(x, y)=(0,0)$ (türkisfarben dargestellt) für die Ausdehnung des Quellgebietes in südöstl. Richtungen verantwortlich. Etwas später (in Abschn. 3.4.2) wird deutlicher werden, dass es genau in diesem Bereich (violett-blau dargestellt) zu einem Kanalisierungseffekt kommt; dadurch wird die Strömung zunächst langsamer, bevor sie in Richtung Turm strömt.

Noch bedeutsamer ist, dass sich der Einflussbereich, welcher $50 \%$ des Gesamtflusses markiert, und der über homogenem Gelände im Luv des Turmes gelegen ist, sich über dem heterogenen Gelände leicht in den windabgewandten Bereich des Turmes verschoben hat. Dieses Phänomen betrifft alle betrachteten Windrichtungen, wobei Winde aus östlichen Richtungen besonders betroffen zu sein scheinen (Abb. b,d und f). Zwar wurden - nach Ermessen des Autors - bis jetzt noch keine Footprint Untersuchungen dieser $\mathrm{Art}^{3}$ angestellt, sodass vergleichbare Studien fehlen; es ist aber beispielsweise auch aus zweidimensionalen Quellgebietsuntersuchungen bekannt, dass es auf der windabgewandten Seite einer Relieferhöhung bzw. eines Hügels, zu u.U. signifikantem Flussbeitrag durch stationäre Verwirbelungen und turbulent-diffusive Transportvorgänge kommen kann [SPGV05]. Der Einflussbereich der verschiedenen Windrichtungen liegt also bei komplexen Gelände nicht ausschließlich im Luv vom Messort, wie z.B. alle analytischen Modelle über flachem Gelände nahelegen, sondern auch im Lee. Weiterhin Bemerkenswert: Die charakteristischen Modifizierungen der einzelnen Quellgebietsbereiche durch reliefbedingte Ablenkung der Strömungslinien. Sowohl Abb. 3.7 (a) als auch Abb. 3.7 (c) sind im Vergleich stärker nach West geneigt. Offensichtlich bewirkt die flankierende Topographie eine Ablenkung der Strömung nach innen. Die Frage, warum die Quellgebiete Abb. 3.7 (d,f) indes etwas weiter ausgedehnt sind, lässt sich wahrscheinlich mit veränderten Strömungsgeschwindigkeiten innerhalb des Anströmfeldes beantworten. Insgesamt sind die Unterschiede aber unbedeutend. Ferner lässt die aufgrund der langen Rechenzeiten recht gering gewählte Auflösung des Gitters von 100x100 $m^{2}$ eine detailliertere Darstellung der Quellgebiete nicht zu.

Da der Vergleich des Einflussbereichs über horizontalem Gelände mit dem über komplexem Gelände, erwartungsgemäß zu keinen größeren Unterschieden bezüglich seiner luvseitigen Ausdehnung führte, scheint es gerechtfertigt, eine Footprintanalyse für stabile Schichtungsverhältnisse anzuschließen. (Unter stabilen atmosphärischen Schichtungsbedingungen ist die Quellgebietsausdehnung am größten.) Auf Abb. 3.8 sind die zwei Fälle, berechnet nach

\footnotetext{
${ }^{3}$ Bei den von Sogachev selbst durchgeführten Untersuchungen handelt es sich ausschließlich um 3-d Untersuchungen ohne Orographieeinfluss. Ref.: z.B. [SRV04].
} 

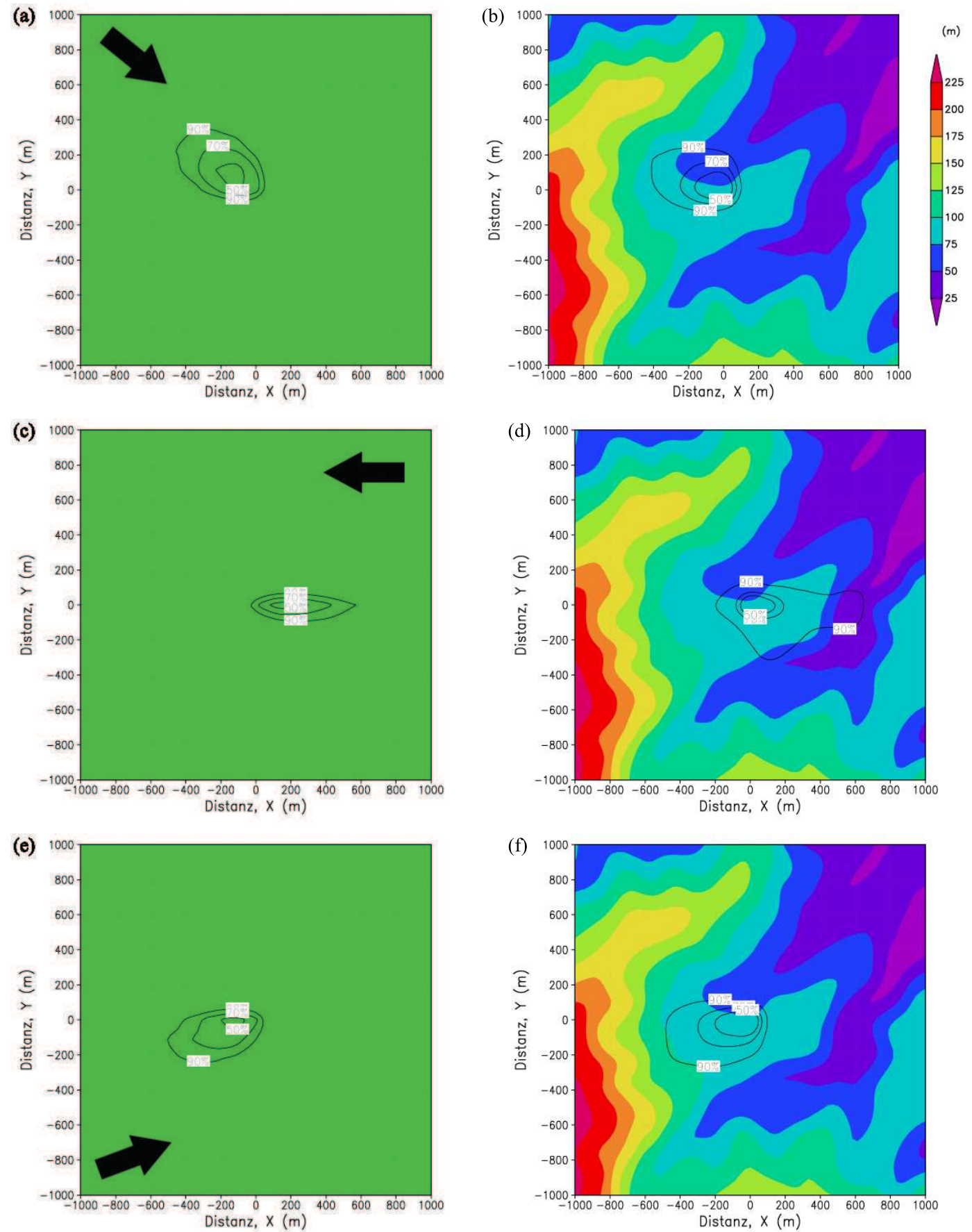

Abbildung 3.7: Quellgebietsanalyse für den Nettofluss über flachem und über komplexem Gelände. Der Turmstandort befindet sich im Koordinatenursprung $(0,0)$. Die Abbidungen a, c und e stellen die Quellgebietsbereiche über homogener und die Abbildungen b,d und f über realer $\left(z=48 m, z_{0}=2.7 \mathrm{~m}\right)$ Orographie (farbige Bereiche) dar. Dargestellt sind die 50, 70 und 90\% Isoplethe (siehe Bezeichnungen auf den Abbildungen) der Quellgebietsfunktion. Die vorgegebene Geostrophische Windgeschwindigkeit beträgt $G=10 \mathrm{~m} \mathrm{~s}^{-1}$. 


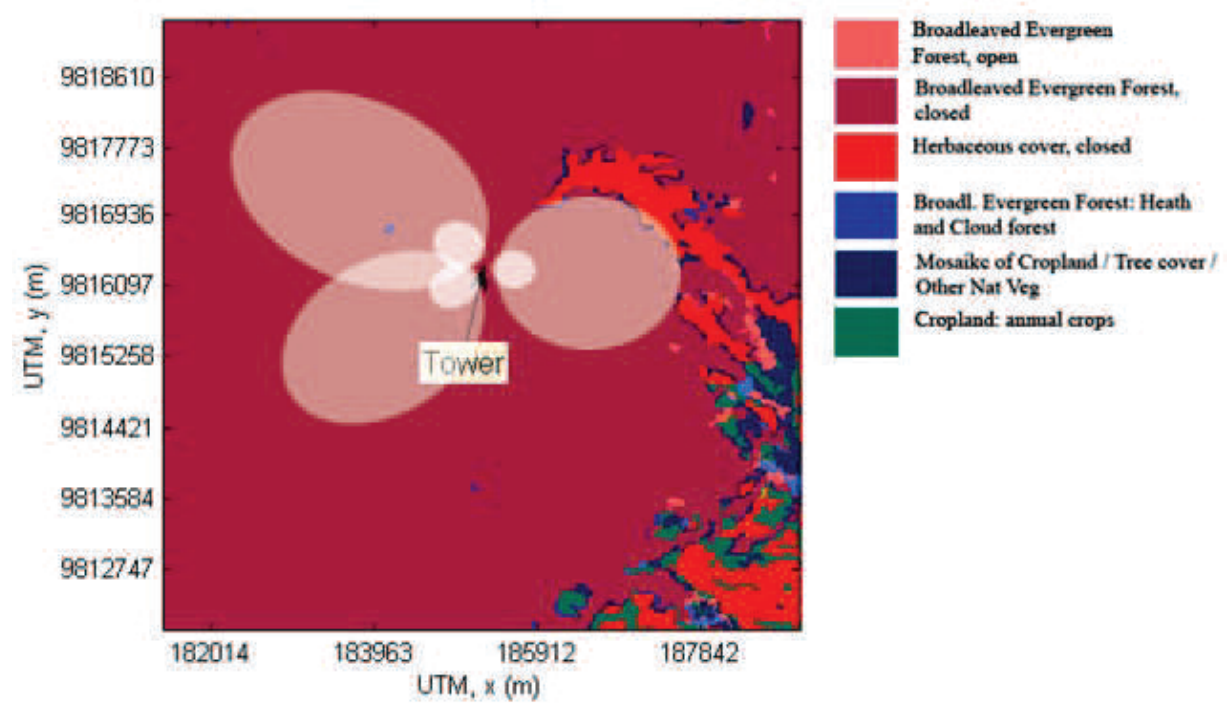

Abbildung 3.8: Quellgebietsanalyse mit dem analytischen Footprint-Modell nach [KM01]. Die dargestellten Quellgebiete (Versatzhöhe: $d=27.3 m$, Rauhigkeitslänge: $z_{0}=2.7 \mathrm{~m}$ ) berücksichtigen $90 \%$ des gemessenen Flusses der Hauptwindrichtungen im Luv des Messturmes für jeweils stabile und neutrale Schichtung.

dem KM-Modell, in unterschiedlicher Transparenz dargestellt. Etwa 2-3km östlich vom Messturm wechselt die Vegetation. Hier schließt sich vorwiegend geschlossene Kräuterwiese an, und unter der Annahme vernachlässigbarer Orografieeinflüsse zeigt sich, dass die Bedingung eines homogenen Anströmgebietes verletzt sein könnte. In Abschnitt 3.1.1 hat sich jedoch gezeigt, dass die Nachtflüsse beinahe ausschließlich aus WSW-lichen Richtungen $\left(\approx 240^{\circ}\right)$ kommen, sodass sich die Situation dadurch entschärft.

\subsubsection{Untersuchung schichthöhenkonstanter Flüsse}

Turbulente Flüsse werden demnach nicht nur durch den Quellgebietsbereich, mit dem die Luft in Wechselwirkung steht, beeinflusst, sondern auch durch sprunghaft veränderte Unterlageneigenschaften. Nach einem Rauhigkeitssprung oder veränderten thermischen Eigenschaften ${ }^{4}$ hat sich die atmosphärische Oberflächenströmung den neuen Rauhigkeitsverhältnissen anzu-

\footnotetext{
${ }^{4}$ So führt komplexe Orografie beispielsweise zu veränderten Einstrahlwinkeln der Sonnerstrahlung und dadurch zu einer heterogenen Temperaturverteilung bezüglich der Unterlage.
} 
Tabelle 3.1: Ellipsenparameter (siehe Abb. 2.11) aus dem KM-Modell zur Darstellung der einzelnen Quellgebietsbereiche (vergl. Abb. 3.8). Es wurden entsprechend der drei Hauptwindrichtungen zwei atmosphärischen Stabilitäten (neutral/stabil) berechnet.

\begin{tabular}{lrrrrrrrrrr}
\hline Windrichtung $\left(^{\circ}\right)$ & 90 & \multicolumn{1}{c}{240} & \multicolumn{7}{c}{300} \\
\hline Ellipsenparameter $(m)$ & $\mathrm{a}$ & $\mathrm{b}$ & $\mathrm{c}$ & $\mathrm{a}$ & $\mathrm{b}$ & $\mathrm{c}$ & $\mathrm{a}$ & $\mathrm{b}$ & $\mathrm{c}$ \\
\hline \hline stabil & 56 & 2339 & 972 & 44 & 2702 & 901 & 54 & 3344 & 993 \\
neutral & 14 & 643 & 210 & 5 & 683 & 236 & 10 & 614 & 276 \\
\hline
\end{tabular}

passen, sodass sich das Profil des Windes und der transportierten Skalare in einem Übergangsprozess befindet. In der Folge sind die turbulenten Flüsse innerhalb der Prandtl-Schicht nicht mehr höhenkonstant - eine Voraussetzung für die Anwendung der EK-Methode.

Zur Überprüfung der Höhenkonstanz wurden der $\mathrm{CO}_{2}$-Fluss, die Schubspannungsgeschwindigkeit $u_{*}$ sowie der sensible $(H)$ und der fühlbare $(\lambda E)$ Wärmestrom aus zwei verschiedenen Höhen miteinander verglichen (siehe Abb. 3.9). Die zweite Messapparatur wurde $8 m$ über der stationären in $28.7 m$ Versatzhöhe (displacement height) montiert. Insgesamt wurde den gesamten Monat Dezember 2004 kontinuierlich und synchron in den zwei Höhen (48m und $56 \mathrm{~m}$ ) gemessen. Alle Abweichungen liegen unter dem zugelassenen Restfehler von 10\%. Die Abweichung der $\mathrm{CO}_{2}$-Flüsse beträgt $7.7 \%$ und bei $\mathrm{H}$, $\lambda E$ und $u_{*}$ wurde der Restfehler sogar deutlich $(<5 \%)$ unterschritten (vergl. Abb. 3.9).

\subsubsection{Test zur integralen Turbulenzcharakteristik}

Eine weitere bedeutende Methode zur Untersuchung des Standorts stellt die sogenannte integrale Turbulenzcharakteristik dar [PTLW77]. Sind die gemessenen Werte der integralen Turbulenzstatistik deutlich höher als das Modell vorgibt, kann zusätzliche mechanische Turbulenz, wie sie von Geländeinhomogenitäten ausgeht, der Auslöser sein [FW96]. Entsprechend den Empfehlungen von Foken \& Wichura [FW96] ist das Modell in Abb. 3.10 mit $\pm 30 \%$ Schranken (unterbrochene Linien) zur Markierung des Toleranzbereichs versehen worden.

Als Resultat ergibt sich für die zwei Höhen aus Abschnitt 3.3.3, dass in $48 m$ lediglich $7.6 \%$ und $56 \mathrm{~m}$ lediglich $2.4 \%$ aller Daten außerhalb der Schranken 


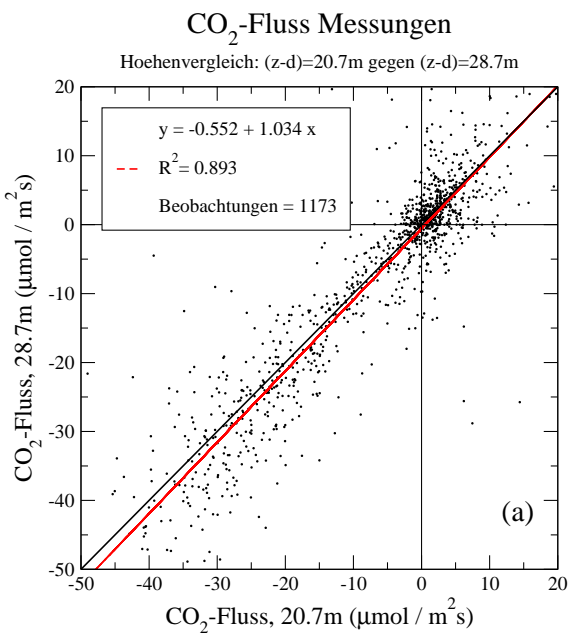

Fuehlbarer Waermestrom, $\mathrm{H}$

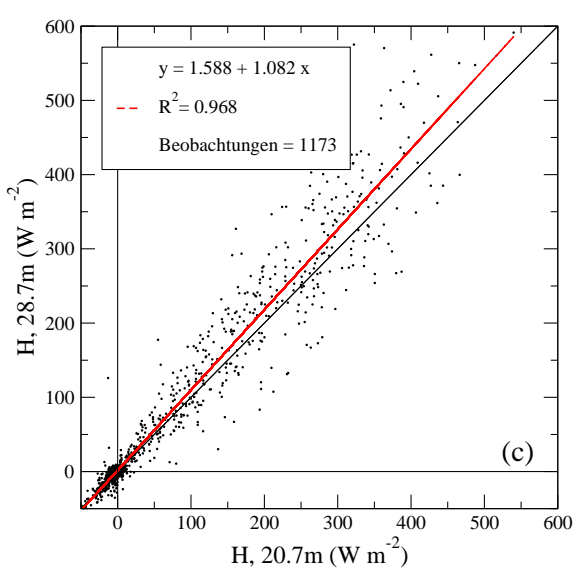

Schubspannungsgeschwindigkeit, $\mathrm{u}_{*}$

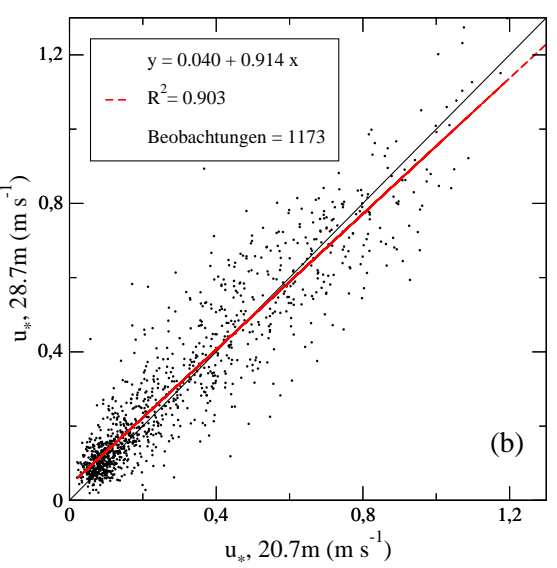

Latenter Waermestrom, $\lambda \mathrm{E}$

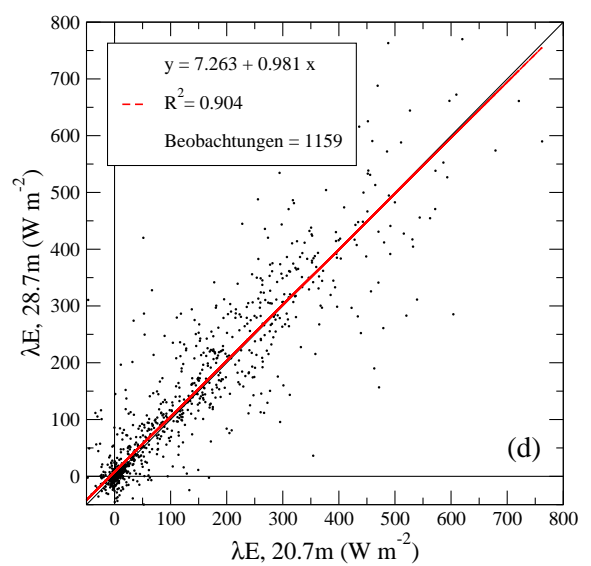

Abbildung 3.9: Überprüfung der Schicht höhenkonstanter Flüsse: Der $\mathrm{CO}_{2^{-}}$ Fluss $\left(F_{C}\right)$, die Schubspannungsgeschwindigkeit $\left(u_{*}\right)$ (als Quadratwurzel des Impulsflusses $\sqrt{\left|\overline{u^{\prime} v^{\prime}}\right|}$ ) und der sensible $(H)$ respektive der latente Wärmefluss $(\lambda E)$ aus zwei Höhen $(z-d=20.7 \mathrm{~m}$ respektive $28.7 \mathrm{~m})$ ist jeweils gegeneinander dargestellt. 

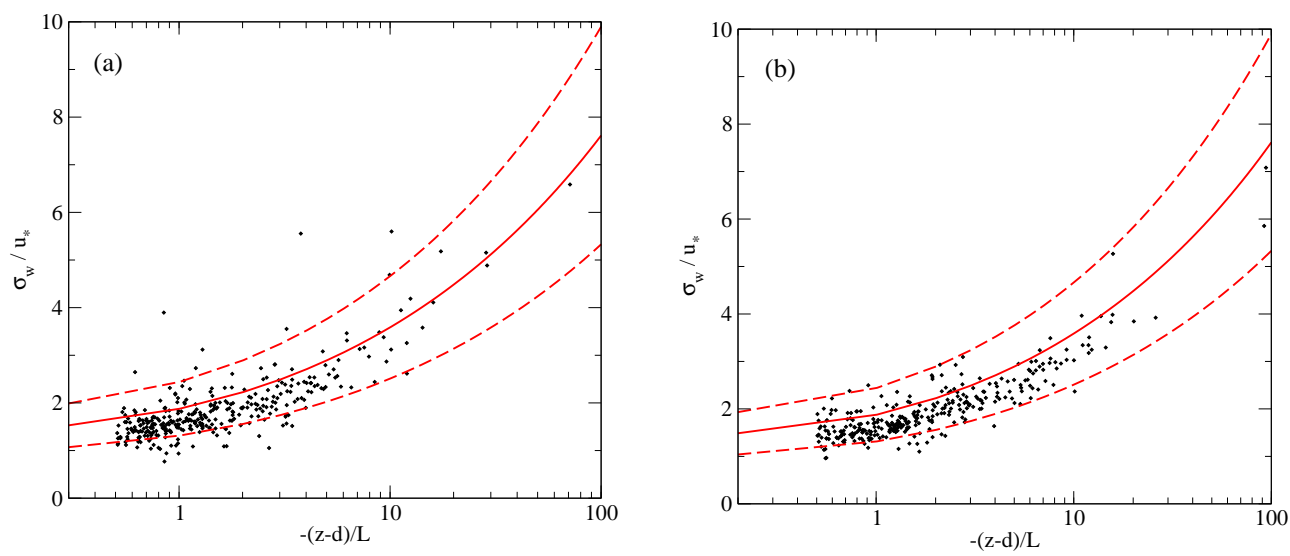

Abbildung 3.10: Qualitätstest der integralen Turbulenzcharakteristik nach dem Modell von Panofsky et al. [PD88]. Dargestellt ist die normierte Standardabweichung der Vertikalwindkomponente für (a) $48 m$ und (b) $56 m$ über dem Bodenrelief. Die unterbrochenen Linien geben $\pm 30 \%$ Schranken an, wie sie von Foken und Wichura [FW96] zur Markierung des Toleranzbereichs vorgeschlagen wurden.

liegen. In $48 m$ Höhe sind die Werte etwas zentraler um den modellierten Kurvenverlauf verteilt gelegen; signifikante Ausreißer blieben aus.

\subsubsection{Stationaritätstest und $u_{*}$-Filterkriterium}

Wie in Abschnitt 2.1.7.1 bereits erläutert wird mit dem Stationaritätstest die wichtige Bedingung zeitlich konstanter Zeitreihen gewährleistet.

Abb. 3.11 zeigt die prozentuale Häufigkeitsverteilung der als instationär gewerteten $\mathrm{CO}_{2}$-Füsse in Abhängigkeit vom Stabilitätsparameter ऽ. Beinahe $35 \%$ der Instationaritäten liegen im neutralen respektive nahe neutralen Schichtungsbereich zwischen $\varsigma \pm 0.1$. Das ist erstmal nicht verwunderlich, denn die rel. Häufigkeit der Instationaritäten spiegelt lediglich die rel. Häufigkeit von $\varsigma$ selber (kleine Grafik Abb. 3.11) wider.

Grundsätzlich sind neutrale Schichtungsverhältnisse in der Regel mit den Tag-Nacht Übergangszeiten oder starker Bewölkung in Verbindung mit stürmischen Verhältnissen verknüpft [Ibr01]. Da stürmische Verhältnisse am Standort Bariri vernachlässigbar selten aufgetreten sind, sollten ausschließlich die Übergangszeiten von Bedeutung sein. Offensichtlich stellt der sich 
Tabelle 3.2: Resultate aus den Qualitätstests für die gesamte Messperiode.

\begin{tabular}{lrr}
\hline Daten & Obs. $(N)$ & rel Abw. $(\%)$ \\
\hline \hline Daten insgesamt & 28825 & 100 \\
techn. Probleme & 6428 & 22.3 \\
schwere Instationaritäten & 1816 & 6.3 \\
moderate Stationaritätsprobleme & 8388 & 29.1 \\
keine Stationaritätsprobleme & 12193 & 42.3 \\
\hline Nachtdaten $\left(R_{G}<10 \mathrm{Wm}^{-2}\right)$ & 6032 & 100 \\
sehr niedriger Turbulenzgrad $\left(u_{*}<0.2\right)$, Nacht & 5218 & 86.5 \\
hoher Turbulenzgrad $\left(u_{*}>0.3\right)$, Nacht & 814 & 13.5 \\
\hline
\end{tabular}

morgend- bzw. abendlich vollziehende Quellgebietswechsel eine ebenso bedeutende Ursache für Instationaritäten dar, wie in der Literatur häufiger vorkommende unterdrückte Austauschprozesse. Beispielsweise fand Rebmann [Reb03] an einem Mittelgebirgsstandort im Fichtelgebirge, der eine ganz ähnliche Häufigkeitsverteilung von $\varsigma$ aufwies, dass die rel. Verteilung der Instationaritäten eine ganz entgegengesetzte Form zeigte. Dort konnten die vergleichsweise häufig auftretenden Instationaritäten bei stabiler Schichtung plausibel auf unterdrückte Austauschprozesse, verbunden mit blasenartiger Ablösung von vorher im Stammraum akkumuliertem Kohlendioxid zurückgeführt werden. Da unser Standort ebenfalls stark von unterdrückter Turbulenz bei Nacht betroffen ist, erstaunt es schon, dass Instationaritäten in diesem Bereich vergleichsweise selten vorkommen. Als wahrscheinlichstes Erklärungsmuster bieten sich die im vorherigen Abschnitt 3.1.1 beschriebenen katabatische Schwereflüsse (Gravity Flows) an, die das respirierte $\mathrm{CO}_{2}$ in der Nacht hangabwärts - am Sensor vorbei - abzuführen scheinen.

Abschließend ist zu konstatieren, dass uns mit 98.6\% (vergl. Tab. 3.2) ein beinahe vollständiger Datensatz über die Messperiode gelungen ist. Von den verfügbaren Daten verblieben $77.7 \%$ nach Abzug verfälschter Daten - zum überwiegenden Teil verursacht durch Regen, der die Funktion des IRGA's störte. Schwere Stationaritätsprobleme traten nur bei etwa $6.3 \%$ der verfügbaren Daten auf.

Nach Bereinigung der Daten wurde das $u_{*}$ - Kriterium entsprechend der Prozedur aus Abschn. 2.2.2 angewendet. Dabei stellte sich sehr schnell heraus, dass viele Schritte aus denselben Gründen, wie den in Abschn. 2.2.2 bzgl. der Modellierung der nächtlichen Respiration bereits erläutert, bei unserem Datensatz entfallen. Zunächst wurde zwischen einer etwas regnerischen (erste Jahreshälfte 2004) und einer etwas trockeneren Phase (zweite Jahreshälfte 


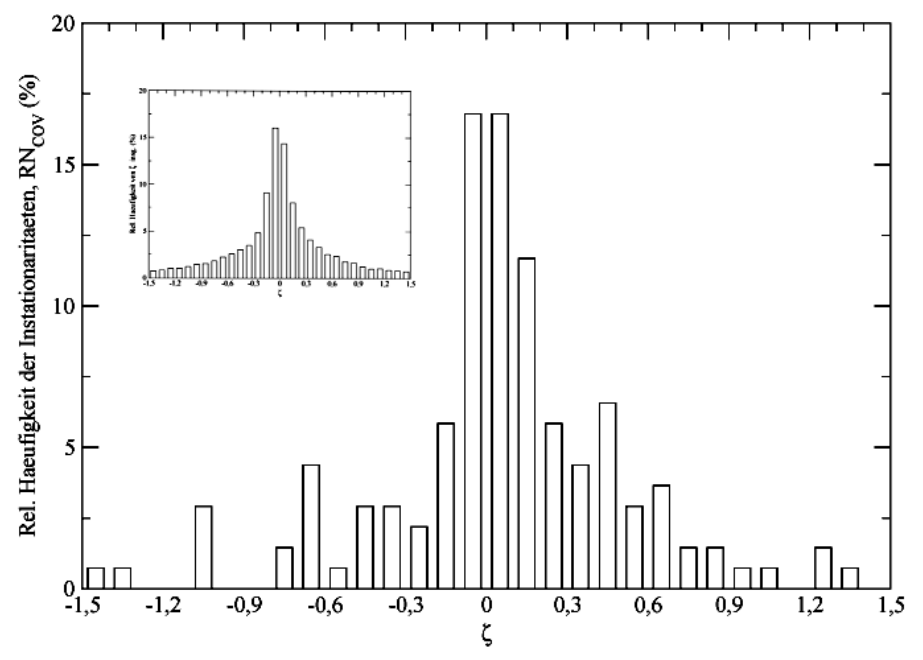

Abbildung 3.11: Relative Häufigkeit von instationären $\mathrm{CO}_{2}$ Halbstundenzeitreihen und Häufigkeitsverteilung aller $\mathrm{CO}_{2}$-Flüsse insgesamt (siehe Abb. in der Abb.) in Abhängigkeit des Stabilitätsparameters $\zeta$.

2004) unterschieden (vergl. Abb. 3.4). Einschränkend ist zu bemerken, dass die Niederschlagsdaten von einer $2 \mathrm{~km}$ entfernten Meteorologiestation stammen. Es liegt ein zweiter kürzerer Niederschlagsdatensatz vor, der direkt am Turm in $70 \mathrm{~m}$ Höhe gemessen wurde. Hier variiert der saisonale Niederschlagsverlauf etwas deutlicher. Mit durchschnittlichen Niederschlägen von $198 \mathrm{~mm} \mathrm{mon}^{-1}$ während der ersten Jahreshälfte und $117 \mathrm{~mm}$ mon $^{-1}$ im Verlauf der zweiten Jahreshälfte, regnete es demnach von Januar bis Juli etwas weniger als doppelt soviel wie zum Jahresende (Aug.-Dez.) hin [IRK $\left.{ }^{+}\right]$. Nach der saisonalen Trennung stellte sich nun die Frage, inwieweit das Bilden von weiteren Temperaturkassen bei einer nur $4-6^{\circ} C$ breiten nächtlichen Temperaturvariation notwendig und sinnvoll erscheint.

Nachdem $T$ und $u_{*}$ auf mögliche Korrelationen überprüft wurden, zeigte sich unmittelbar, dass weitere Klassenbildungen nicht notwendig waren. Beide Korrelationskoeffizienten lagen betraglich deutlich unter 0.2. Damit lagen die Werte klar unter der geforderten Akzeptanzschwelle von 0.4 (vergl. Abschn. 2.2.2). Gewählt wurde schließlich der Datensatz der zweiten Jahreshälfte, da hier die Schwelle $u_{* t h r e s}$ mit einem Wert von $0.3 \mathrm{~m} \mathrm{~s}^{-1}$ etwas höher lag (vergl. Abb. 3.12 a). Abbildung 3.12 (b) zeigt die prozentuale Verteilung der zur Darstellung von Abb. 3.12 (a) verwendeten Nachtflüsse als Funktion der 
Schubspannungsgeschwindigkeit. Nicht gewertet wurden normalisierte Flüsse ab $u_{*}>0.7 \mathrm{~m} \mathrm{~s}^{-1}$, da die Unsicherheit aufgrund der stark reduzierten Zahl an Observationen zu stark ansteigt. Damit verblieben 13.5\% der Nachtflussdaten mit ausreichendem Turbulenzgrad. Der konstante Verlauf des normalisierten $\mathrm{CO}_{2}$-Flusses deutet darauf hin, dass advektive Prozesse für $u_{*}>0.3 \mathrm{~ms}^{-1}$ stärker unterdrückt werden $\left[\mathrm{IRK}^{+}\right]$.

\subsubsection{Energiebilanzschließung}

Die Energiebilanzschließung stellt ein weiteres Kriterium für die Plausibilität von EK-Messungen dar $\left[\mathrm{AGI}^{+} 00\right]$. Sie gilt nach dem ersten Hauptsatz der Thermodynamik und drückt sich in einer geschlossenen Bilanz aller in den Bestand hinein- und hinausfließenden Energieströme aus.

Nach der Energiebilanzgleichung gilt:

$$
R_{n}-G_{t}=H+\lambda E .
$$

$R_{n}$ bezeichnet die Nettostrahlung, $G_{t}$ die Änderungsrate der im Wald und der Bestandesluft gespeicherten Energie, $H$ die fühlbare und $\lambda E$. die latente Wärme. $H$ und $\lambda E$ wurden mittels der Daten aus den EK-Messungen berechnet. Die Differenz $R_{n}-G_{t}$ wird auch als verfügbare Energie bezeichnet. Da $G_{t}$ im Rahmen der Untersuchungen nur teilweise erfasst werden konnte, wurde allein $R_{n}$ berücksichtigt. Das ist möglich, wenn Tagesmittel gerechnet werden, denn in diesem Fall kann $G_{t}$ als vernachlässigbar klein angenommen werden.

Auf Abbildung 3.13 sind die turbulenten Wärmeströme gegen die verfügbare Energie aufgetragen. Die Schließung beträgt ungefähr 90\%. Aus einer Schließung dieser Qualität sollte sich mit etwas Vorsicht ableiten lassen, dass signifikante Flussbeiträge berücksichtigt wurden. Allerdings besteht bei der Betrachtung von Tagesmitteln die Gefahr, sich kompensierende Fehler, die im Verlauf des Tages unter speziellen meteorologischen Bedingungen auftreten können, zu übersehen $\left[\mathrm{AGI}^{+} 00, \mathrm{IRK}^{+}\right]$.

\subsubsection{Die Planar-Fit Methode}

Nach den Ausführungen aus Abschn. 2.1.6 basiert die Planar-Fit Methode auf einer Reihe theoretischer Voraussetzungen. Eine der wichtigsten besteht in der Annahme, dass die Strömungslinien bei neutraler Schichtung 

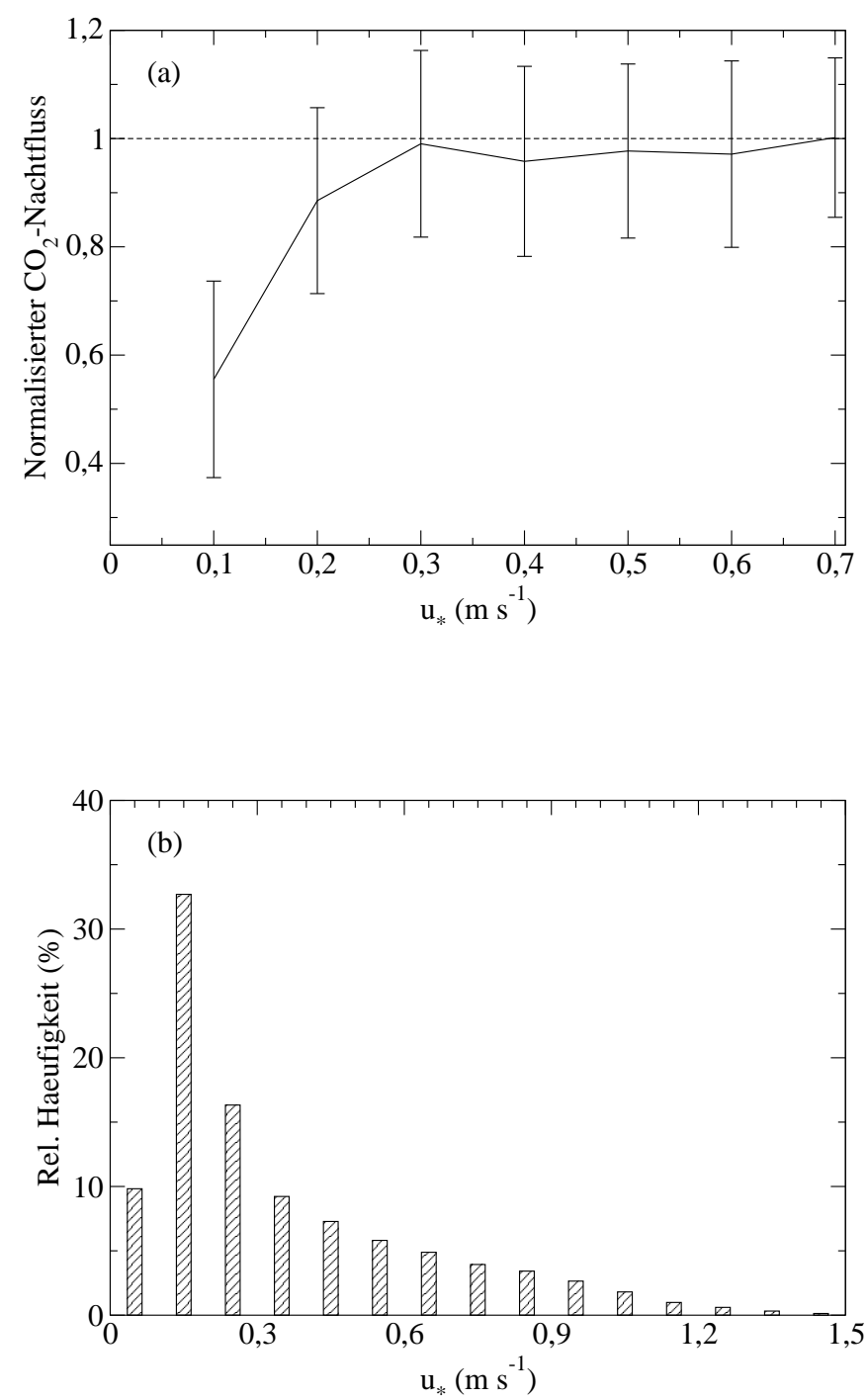

Abbildung 3.12: (a) Normalisierte $\mathrm{CO}_{2}$-Nachtflüsse $\left(F_{C} / F_{C \max }\right)$ gemittelt über sich überlappende Unterdatensätze der Fensterbreite $0.2 \mathrm{~m} / \mathrm{s}$ und (b) die relative Häufigkeitsverteilung der verfügbaren Daten in Abhängigkeit von $u_{*}$. 
Energiebilanzschliessung

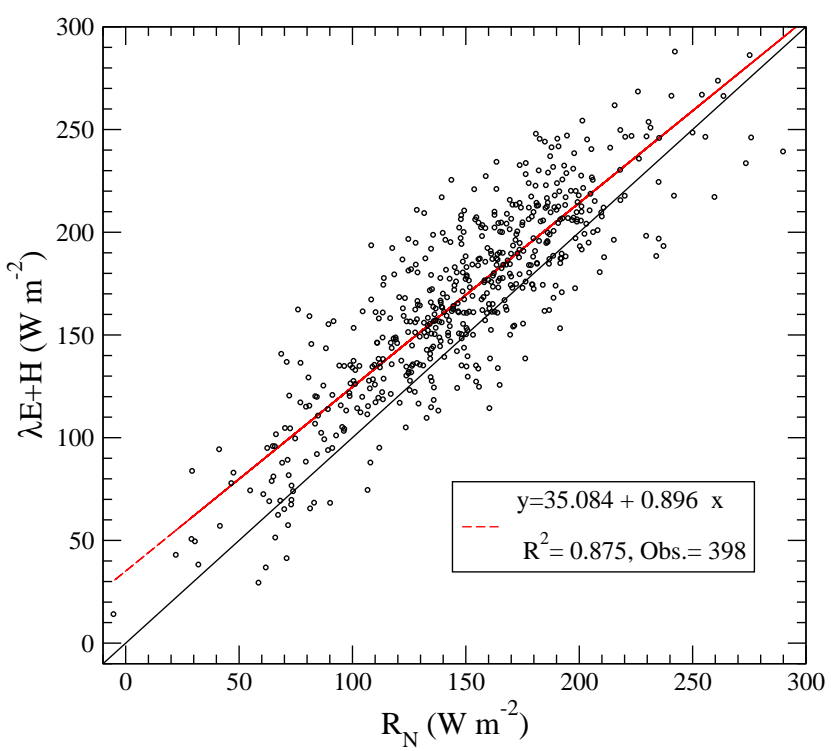

Abbildung 3.13: Tagesmittel der turbulent transportierten Energie als Funktion der verfügbaren Energie für das Jahr 2004. Lediglich Tage, an denen mehr als 24 Halbstundenstatistiken zu Verfügung standen, wurden einbezogen. (Regression: rot, unterbrochene Linie, 1:1-Linie: schwarz)

der Orografie folgen sollten (vergl. Abb. 3.14 a). Um einen Eindruck von dem Verlauf der Strömungslinien in Abhängigkeit von der Orographie zu bekommen, wurde das dreidimensionale Geschwindigkeitsfeld für Westwind berechnet. Abbildung 3.14 (a) zeigt den modellierten Verlauf der Strömungslinien im Reliefschnitt von West nach Ost. Null bezeichnet den Turmstandort und der geostrophische Wind wurde mit $10 \mathrm{~m} \mathrm{~s}^{-1}$ vorgegeben $^{5}$ (siehe Pfeil), was in Messhöhe am Turm (48m) Horizontalwindgeschwindigkeiten von ca. $2-3 m s^{-1}$ bedeutet. Zur Darstellung der Stromlinien im kartesischen Koordinatensystem wurde die folgende einfache Funktion verwendet:

$$
\psi(x, z)=\int_{0}^{z} u(x, z) d z .
$$

Anhand der Graphik lassen sich sehr anschaulich die relevanten Maßstäbe entnehmen. Offensichtlich reagiert das Strömungsregime sehr direkt auf die

\footnotetext{
${ }^{5}$ Der geostrophische Wind wurde aus vertikalen Radiosondenprofilen der Wetterbehörde in Palu/Zentralsulawesi abgeschätzt. Eines dieser Profile wurde in unserer Gegenwart (Ballonaufstieg) aufgenommen.
} 
Tabelle 3.3: Planar-Fit Ebenen Winkel $\alpha$ (Neigungungswinkel in WO Richtung) und $\beta$ (Neigungswinkel in NS Richtung) in Abhängigkeit von der Windrichtung und von der Windgeschwindigkeit.

\begin{tabular}{lrrrrr}
\hline Sektor $\left(^{\circ}\right)$ & $U$-Bereich $(\mathrm{m} / \mathrm{s})$ & $\alpha\left(^{\circ}\right)$ & $\beta\left(^{\circ}\right)$ & $R^{2}$ & Obs. \\
\hline $20-160$ & ges. & $0.858 \pm 0.216$ & $1.054 \pm 0.128$ & 0.150 & 503 \\
$20-160$ & $<=1.5$ & $1.971 \pm 0.487$ & $2.070 \pm 0.273$ & 0.177 & 341 \\
$20-160$ & $>1.5$ & $-0.369 \pm 0.335$ & $1.149 \pm 0.144$ & 0.051 & 215 \\
$200-270$ & ges. & $1.660 \pm 0.098$ & $4.245 \pm 0.097$ & 0.450 & 1643 \\
$200-270$ & $=1.5$ & $1.548 \pm 0.185$ & $4.224 \pm 0.217$ & 0.302 & 1040 \\
$200-270$ & $>1.5$ & $1.603 \pm 0.242$ & $4.123 \pm 0.288$ & 0.269 & 556 \\
$270-320$ & ges. & $2.263 \pm 0.057$ & $2.113 \pm 0.080$ & 0.457 & 2107 \\
$270-320$ & $<=1.5$ & $1.685 \pm 0.335$ & $2.905 \pm 0.373$ & 0.219 & 483 \\
$270-320$ & $(1.5,3]$ & $2.190 \pm 0.301$ & $3.523 \pm 0.269$ & 0.326 & 1113 \\
$270-320$ & $>3$ & $3.731 \pm 0.358$ & $4.422 \pm 0.326$ & 0.392 & 718 \\
\hline
\end{tabular}

gegebenen Geländevariationen $\left[\mathrm{RIS}^{+}\right]$, sodass vor allem die näheren Topografieeinflüsse (die ersten $100-200 m$ vor dem Turm) von Bedeutung sein sollten.

Zur Überprüfung und zur Bestimmung der Referenzebene des für die Flüsse zugrunde gelegten Koordinatensystems wurden den drei Hauptanströmrichtungen entsprechend drei verschiedene Windrichtungssektoren zugeordnet. Den Ergebnissen aus Tabelle 3.3 folgend wurde außerdem eine Abhängigkeit der Winkel der Planar-Fit-Ebene von der Windgeschwindigkeit festgestellt. So verändert sich z.B. der Ebenenwinkel $\alpha$ (Neigungungswinkel in WO Richtung) von $\approx 2^{\circ}$ für Windgeschwindigkeiten $\leq 1.5 \mathrm{~m} \mathrm{~s}^{-1}$ nach $\approx-0.3^{\circ}$ für Windgeschwindigkeiten $>1.5 \mathrm{~m} \mathrm{~s}^{-1}$. Offensichtlich entspricht $\alpha=2^{\circ} \mathrm{sehr}$ gut den näheren Geländebedingungen (vergl. Abbildung 3.14 b (WO) im Bereich $0-50 \mathrm{~m})$, während $\alpha=-0.3^{\circ}$ gut mit dem ferneren Gelände $(200-250 \mathrm{~m})$ übereinstimmt. Ganz ähnlich lässt sich auch bzgl. der Windrichtungen aus WNW oder SW argumentieren (vergl. auch Abb. 2.4). Als wesentlich konstanter gegenüber Variationen in der Windgeschwindigkeit erweist sich der Ebenenwinkel $\beta$ (Neigungswinkel in NS Richtung). Auch hier liegt die Begründung in der Neigung des Reliefs. Es verläuft von Nord nach Süd auf etwa 350 Metern Länge $(-200 m$ bis $150 m)$ sehr viel kontinuierlicher und konstanter als das bei der WO-Richtung der Fall war. Dadurch wird sowohl das nähere als auch das fernere Strömungsregime in gleicher Weise beeinflusst $\left[\mathrm{TAB}^{+} 03\right]$. 


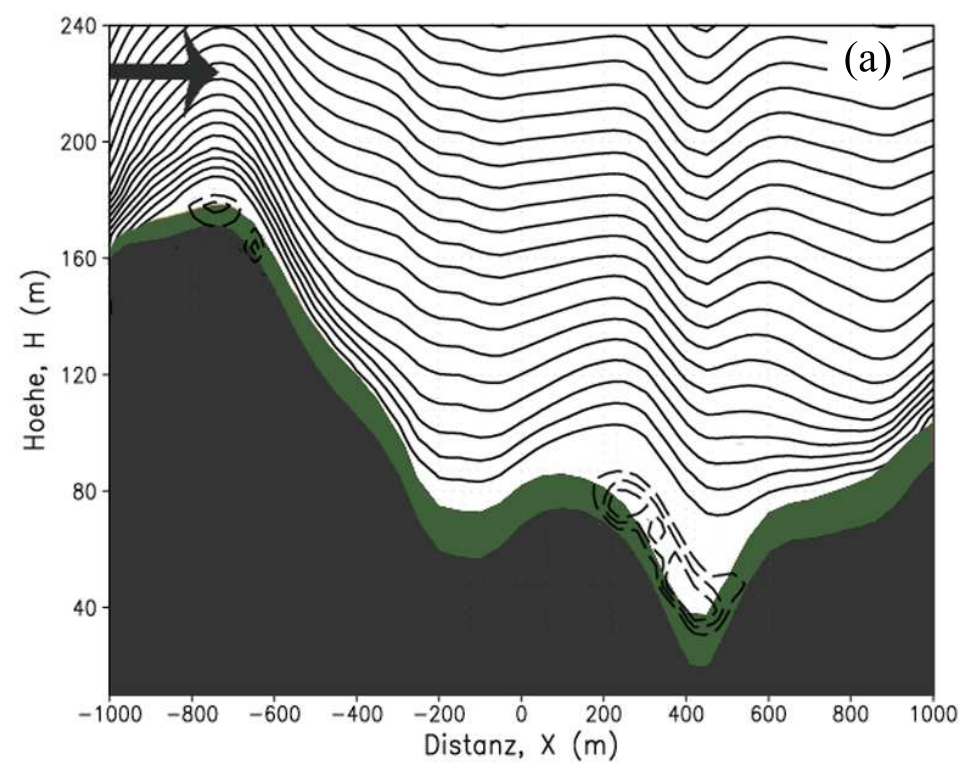

(b)

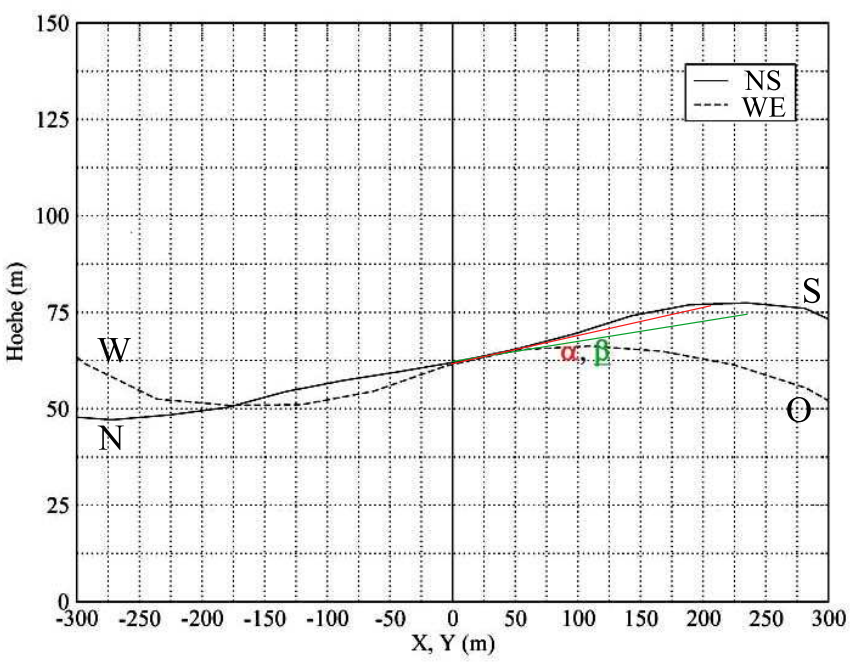

Abbildung 3.14: (a) Verlauf der modellierten Strömungslinien am Beispiel eines vertikalen Schnitts durch Strömungsregime und Bodenrelief in WO Richtung. Der Pfeil markiert die Windrichtung und $X=0$ markiert den Turmstandort. (b) Darstellung der Reliefoberfläche im Bereich der Messungen in NS und WO Richtung zur Überprüfung der Planar-Fit Winkel ( $X, Y=0$ bezeichnet den Turmstandort). 


\subsubsection{Zusammenfassung}

Zusammenfassend bleibt festzuhalten, dass sich die Strömung unter bestimmten Umständen (heterogenes Gelände 100 - 200m im Luv der Messungen, Windgeschwindigkeiten $\geq 1.5 \mathrm{~m} \mathrm{~s}^{-1}$ ) leicht von dem lokalen Geländerelief ablöst (streamline separation). Wie dies bereits die Footprint-Untersuchungen nahelegen, tritt dieser Effekt am deutlichsten bei Winden aus östlichen Richtungen auf. Ebenso weisen hier die Bestimmtheitsmaßwerte $R^{2}$ aus der Ebenenberechnung die niedrigsten Werte auf. Da aber hohe Windgeschwindigkeiten aus diesen Richtungen äußerst selten sind (vergl. Abschn. 3.1.1), sollten sich für die Berechnung der Skalarflüsse keine größeren Unsicherheiten ergeben.

Den Footprint-Untersuchungen nach zu urteilen sollte das Quellgebiet der betrachteten turbulenten Flüsse zu nahezu allen Zeiten innerhalb des zulässigen Fetch ${ }^{6}$ liegen. Die einzige Situation, bei der eine Überschreitung des Fetch prinzipiell möglich erscheint (stabile Schichtung, Wind aus östl. Richtungen), scheint unproblematisch, da stabile Schichtung in den allermeisten Fällen mit süd-westlichen Windrichtungen einhergeht (Abschn. 3.1.1). Auf der anderen Seite legen die Footprintuntersuchungen nahe, dass es hinter der Reliefwölbung (z.B. Abb. 3.14) zu Verwirbelungen (separation bubble) kommen kann. Dies hätte Einfluss auf die vertikale Konzentrationsverteilung der verschiedenen Spurenstoffe und mithin auf die $\mathrm{CO}_{2}$-Flüsse. Den Untersuchungen zur Schicht höhenkonstanter Flüsse nach zu urteilen, erscheint der gewählte Standort zur Durchführung von EK-Messungen als gerechtfertigt. Sowohl die Tests zur Flussdivergenz als auch die integrale Turbulenzcharakteristik haben keine weiteren Hinweise ergeben, von denen aus auf eine gravierende qualitative Beeinträchtigung der Flussmessungen geschlossen werden könnte. Auch die 90\%-ige Energiebilanzschließung lässt die turbulenten Flussmessungen am Standort Bariri plausibel erscheinen [AGI $\left.{ }^{+} 00\right]$.

Schwere Instationaritäten traten vergleichsweise selten bei etwa $6.3 \%$ der verfügbaren Daten auf. Ein vergleichsweise ernstes Problem bei der Durchführung von EK-Messungen stellt die nächtlich stabile Schichtung, verbunden mit sehr niedrigen Windgeschwindigkeiten $(<3 \mathrm{~m} / \mathrm{s})$ in den Tropen, dar. Hier kam es zu einem nachträglichen Verlust nächtlicher Daten von $86.5 \%$.

\footnotetext{
${ }^{6}$ Bezeichnet die räumliche Ausdehnung der zu untersuchenden Vegetationschicht.
} 


\subsection{Berücksichtigung advektiver Strömungen}

Nachdem die bisherigen Untersuchungen (vergl. Abschnitt 3.1.1) die Existenz advektiver $\mathrm{CO}_{2}$-Flüsse vor allem bei Nacht sehr nahelegen, soll es mit diesem Abschnitt nun vor allem um zwei Dinge gehen: Zum einen sind die bisherigen Vermutungen im Rahmen der vorhandenen Möglichkeiten zu erhärten und zum anderen wird mit dieser Arbeit das Ziel verfolgt, den Messfehler, der im Fall komplexerer Gelände - so die Vermutung - durch unbeachtete advektive Flüsse immer auftritt, abzuschätzen. Im Folgenden werden also nächtliche und am Tage auftretende Advektionen thematisch getrennt behandelt. Während nächtliche Advektion (Abschn. 3.4.1) über bergigem Gelände in der Regel durch katabatischen Kaltluftströmungen von höchstens einigen 10 Metern Dicke hervorgerufen wird [SK88], ist Advektion am Tage und insbesondere zu den Übergangszeiten (Abschn. 3.4.2) sehr viel direkter durch die Topografie beeinflusst. Eine nächtliche Kaltluftströmung, wie sie im nächsten Abschnitt behandelt wird, fließt in Form einer langsam zunehmenden Schicht talwärts, sodass es zu einer Entkopplung mit dem darüberliegenden Strömungsregime kommen kann. Ein Phänomen, das aus strömungsphysikalischer Sicht tagsüber kaum denkbar ist.

\subsubsection{Abschätzung advektiver Einflüsse bei Nacht (ka- tabatische Schwereflüsse)}

Zur Abschätzung der vertikalen Advektion wird nach dem in [Lee98] beschriebenen Verfahren vorgegangen (vergl. Abschn. 2.1.2.1). Da zur Zeit der Messkampagne nur über einen kurzen Zeitraum $\mathrm{CO}_{2^{-}}$Konzentrationsprofilmessungen zur Verfügung standen, wurde im Rahmen der Untersuchungen auf modellierte Konzentrationsprofile zurückgegriffen. Bei dem verwendeten Modell handelt es sich um ein Soil-Vegetation-Atmosphere-Transfer, kurz SVAT, Modell. Das $1 d$ prozessbasierte Modell, mit Namen Mixfor-SVAT [OCN ${ }^{+} 02$, wurde bereits erfolgreich für Untersuchungen tropischer Vegetationsbestände verwendet [Fal04]. Darüber hinaus wurde das Modell zur Beschreibung der regionalen Dynamik von $\mathrm{CO}_{2}$ - und Wasserdampfflüssen im Untersuchungsgebiet Zentral Sulawesi verwendet und validiert [IOJ $\left.{ }^{+} 07\right]$. Mixfor-SVAT besteht aus einer Reihe miteinander gekoppelter Unterprogramme, die im Wesentlichen:

- den Strahlungstransport innerhalb der Vegetation,

- den vertikalen turbulenten Austausch von Impuls, sensibler Wärme, 
$\mathrm{CO}_{2}$ und Wasserdampf,

- die Transpiration verschiedener Baumarten auf verschiedenen Niveaus der Vegetation,

- die Wasseraufnahme durch das Wurzelwerk und den Wassertransport zum Blattwerk,

- Interzeptions-, Speicher- und Evaporationsprozesse über verschiedene Niveaus innerhalb der Vegetation,

- $\mathrm{CO}_{2}$ - Bodenrespiration, Fotosynthese sowie autotrophe und heterotrophe Respiration

- und den Wasser- und Wärmetransport der Unterlage

beschreiben. Das numerische Lösungsverfahren basiert auf der Schießung der Energie- und Wasserbilanzgleichungen der verschiedenen Vegetationsschichten und in der Summe des gesamten Waldbestandes. Eine ausführlichere Beschreibung mit Bezug auf das Untersuchungsgebiet ist in [IOJ $\left.{ }^{+} 07\right] \mathrm{zu}$ finden.

Abb. 3.15 (a) zeigt modellierte Profile im 4 Stunden Abstand am Beispiel des 01.11.2004. Dabei wird anschaulich das Zusammenspiel zwischen Respiration, Assimilation und den jeweiligen Austauschbedingungen deutlich. Gegen Abend nimmt die Fotosynthese ab und gleichzeitig beginnt sich $\mathrm{CO}_{2}$ im Stammraum anzureichern. Gegen $4.00 \mathrm{~h}$ erreicht die Konzentration ihr Maximum, während sie sich kurz nach Sonnenaufgang aufgrund der forcierten Austauschbedingungen bereits wieder abzureichern beginnt. Damit ergibt sich ein positiver Flussbeitrag (durch den Speicherterm) in den Nacht- und Abendstunden und ein negativer am Morgen - indem $\mathrm{CO}_{2}$ freigesetzt wird. Neben der Speichertermberechnung aus den modellierten Konzentrationsprofilen wurde der Speicherterm zum Vergleich auf eine weitere Art und Weise ermittelt. Die Berechnung basiert auf einer einfachen Ein-Punkt-Messung aus $48 m$ Höhe und wurde bereits in Abschn. 2.2 eingeführt [HKB $\left.{ }^{+} 94\right]$. Die Nachteile der Methode sind offensichtlich; ihre Gültigkeit beschränkt sich auf Zeiten, in denen der turbulente Austausch ausreichend stark ausgeprägt ist. Situationen mit schwachen Windverhältnissen und stark stabiler Schichtung sind problematisch. Nichtsdestoweniger, die Methode lieferte in sehr vielen Fällen Tagesgänge, die sich nur wenig von Speicherflüssen, berechnet nach der Profilmethode, unterschieden $\left[\mathrm{MBH}^{+} 04\right.$, Reb03]. Tatsächlich zeigt der Vergleich der modellierten mit den gemessenen Werten einen hohen Grad an Übereinstimmung (Abb. 3.15 b).

Zur Abschätzung möglicher advektiver $\mathrm{CO}_{2}$-Flüsse ist auf Abb. 3.16 (a) der normalisierte Speicherfluss als Funktion von $u_{*}$ dargestellt. Zur Norma- 


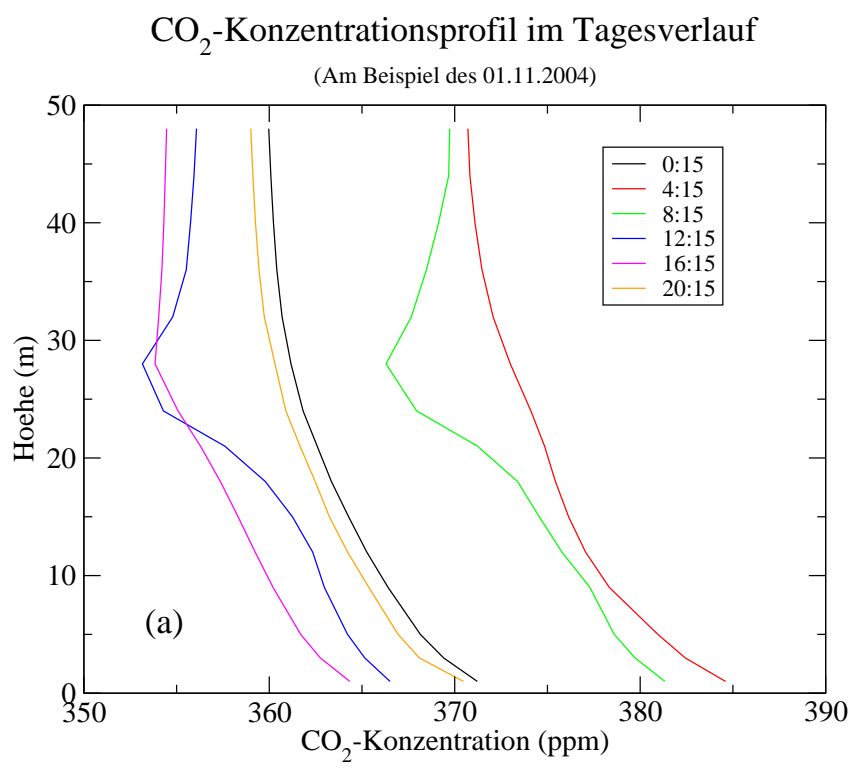

Tagesgang des gemessenen resp. modellierten Speicherterms

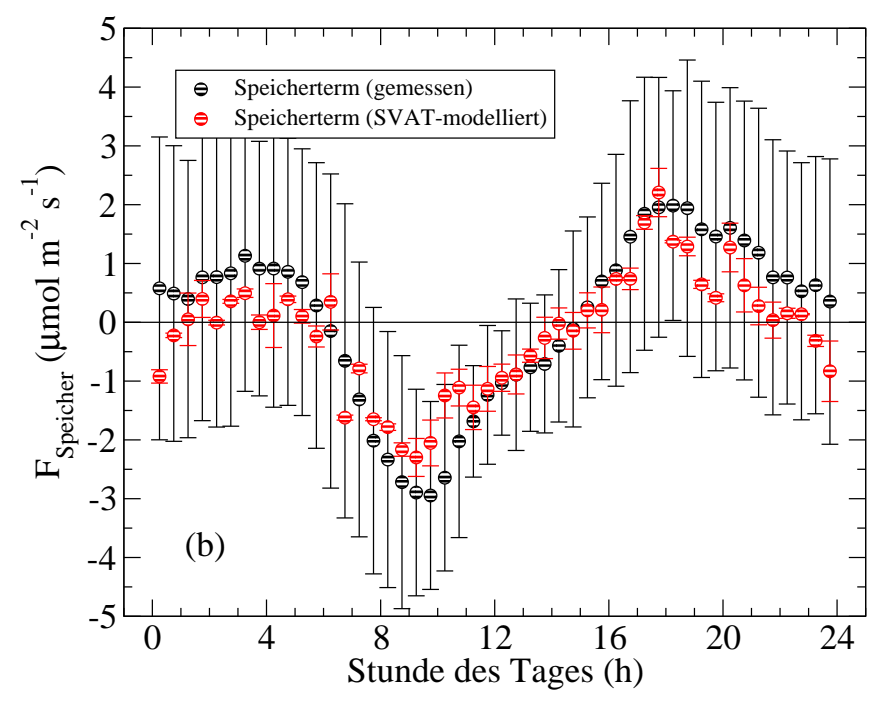

Abbildung 3.15: (a) SVAT-modellierte Konzentrationsprofile am Beispiel des $01.11 .2004 \mathrm{im}$ Tagesverlauf und (b) Vergleich des modellierten mit dem gemessenen (Ein-Punkt-Messung) mittleren Speicherfluss im Tagesgang. Verwendet wurden alle Daten des Jahres 2004. 
lisierung wurde der mittlere Fluss aus Bodenkammermessungen verwendet [Jin06]. Der norm. Speicherfluss, welcher als Anteil von respiriertem $\mathrm{CO}_{2}$, gespeichert innerhalb der Luftsäule unter dem Messsystem, zu interpretieren ist $\left[\mathrm{ABC}^{+} 05\right]$, nimmt auch bei niedrigsten Werten von $u_{*}$ kaum Werte über 0.2 an. Dafür lassen sich schwer plausible Erklärungen finden. Eine Abnahme der Quellstärke aufgrund starker Abkühlung des Bodens (die Temperatur ist ausschlaggebend für die Quellstärke respirativer Flüsse) oder verminderter Diffusion durch Übersättigung $\mathrm{CO}_{2}$-reicher Luft in Bodennähe kann ein so starkes Missverhältnis nicht rechtfertigen. Die einzig plausible Erklärung, warum der Wert auch unter nichtturbulenten Bedingungen derart niedrig bleibt, ist, dass neben den Anreicherungsprozessen im Stammraum auch nichtturbulente Transportprozesse, sprich Advektion, eine Rolle spielen [AHY03]. Da die Bodenkammermessungen vergleichsweise hohe Werte aufwiesen - eine Messkampagne in etwa $50 \mathrm{~km}$ Entfernung ergab mittlere Werte, die $42 \%$ niedriger ausfielen - wurden großzügig Fehlerschranken von $\pm 50 \%$ eingeführt (Abb. 3.16 a, schattierter Bereich), sodass eine mögliche Überschätzung der Flüsse in [Jin06] einbezogen wurde.

Auch der Tagesgang der $\mathrm{CO}_{2}$-Konzentration (Abb. 3.17 a) stützt diese Einschätzung. Zur näheren Erläuterung bietet es sich an, die Untersuchungen am Standort Bariri, mit Untersuchungen, die 1996 von Grace et al. [GML $\left.{ }^{+} 96\right]$ über einem brasilianischen Regenwald durchgeführt wurden, zu vergleichen. Grace et al. konnten zeigen, dass deren Untersuchungsgebiet nicht von advektive Prozessen betroffen ist. Durch die verminderten Austauschbedingungen in der Nacht kam es dort zu großen Speicheränderungen am Morgen, die $-15 \mu \mathrm{mol} \mathrm{m} \mathrm{m}^{-2} \mathrm{~s}^{-1}$ überschritten und $\mathrm{CO}_{2}$ Konzentrationssenkungen von bis zu $100 \mathrm{ppm}$ bewirkten. In der Nacht betrug der Speicherterm im Mittel $5.10 \mu \mathrm{mol} \mathrm{m} \mathrm{m}^{-2} \mathrm{~s}^{-1}$; ungefähr $70 \%$ des respirierten $\mathrm{CO}_{2}$ wurde zwischen $19.00 \mathrm{~h}$ und $5.00 \mathrm{~h}$ im Stammraum gespeichert. Dort reicherten sich in der Nacht mittlere Konzentrationen von 450-550 ppm an, während unsere Untersuchungen Werte ergaben, die $380 \mathrm{ppm}$ nicht übertrafen (siehe Abb 3.17 a). Dagegen zeigt Abb. 3.17 (b), dass der Speicherterm am Standort Bariri nach Sonnenaufgang und einsetzender Turbulenz (also zu einem Zeitpunkt, zu dem die verwendete Methode zur Berechnung des Speicherterms sehr zuverlässige Ergebnisse liefern sollte) kaum Flussbeiträge $<-3 \mu \mathrm{mol} \mathrm{m} \mathrm{m}^{-2} \mathrm{~s}^{-1}$ lieferte. Und auch der (SVAT) modellierte Speicherterm zeigt kaum Nachtwerte $<-2 \mu \mathrm{mol} \mathrm{m} \mathrm{m}^{-2} \mathrm{~s}^{-1}$. So besteht der wesentliche Unterschied zu unseren Messungen darin, dass die nächtlichen turbulenten $\mathrm{CO}_{2}$-Flüsse bei schwachen Winden $\left(u_{*}<0.3 \mathrm{~m} / \mathrm{s}\right)$ vollständig durch den Speicherterm ausgeglichen wurden. Oder anders ausgedrückt: Da das akkumulierte $\mathrm{CO}_{2}$ nicht durch advektive Flüsse abgeführt wurde, bestand für Grace et al. die Möglichkeit, den $N E E$ vollständig zu erfassen. 

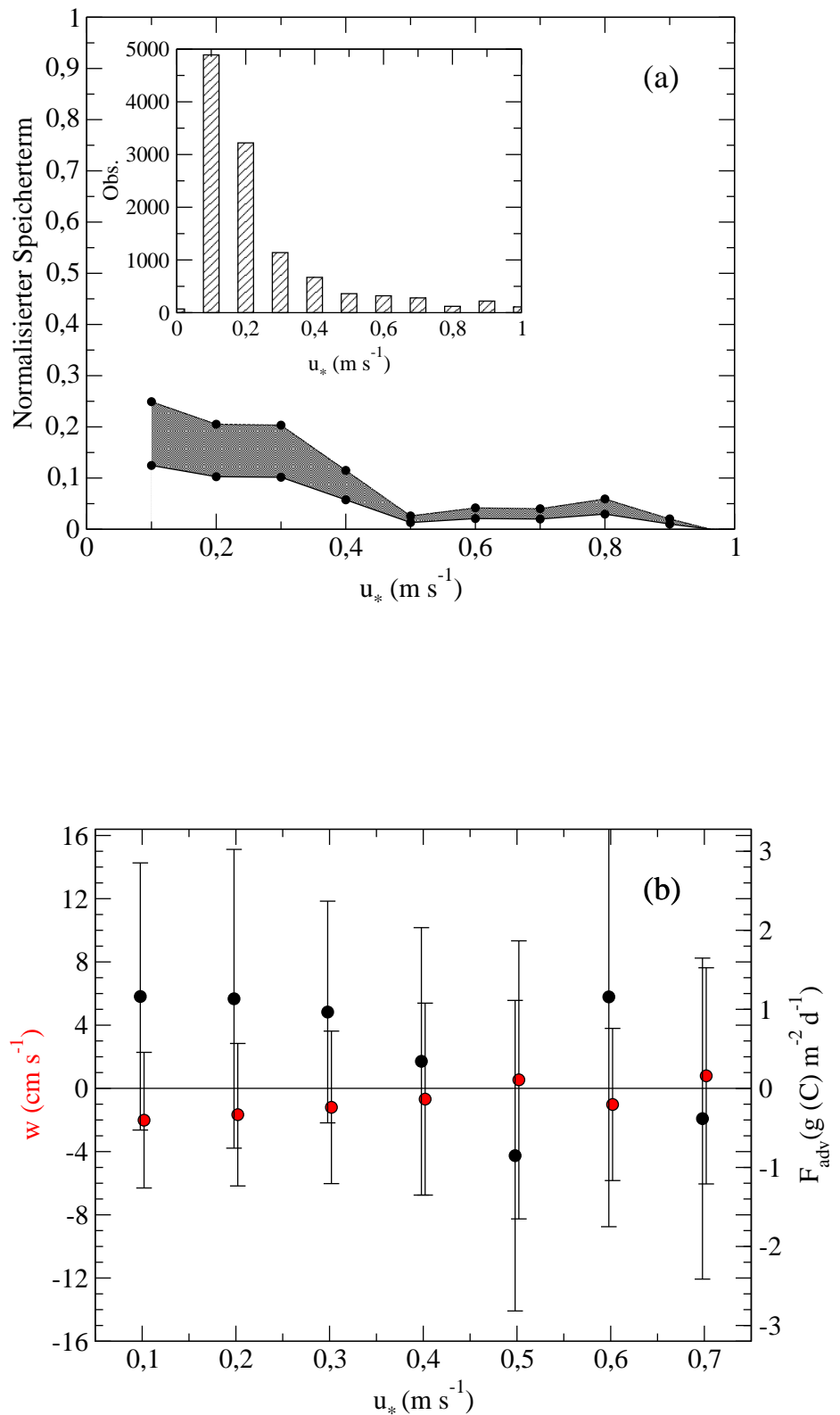

Abbildung 3.16: (a) Normalisierter Speicherterm (wobei der schattierte Bereich den Unsicherheitbereich, wie er durch eine 50\%-ige Überschätzung der Bodenrespiration verursacht würde, darstellt) und (b) vertikaler $\mathrm{CO}_{2}$-Fluss $\left(F_{a d v}\right)$ sowie mittlere Vertikalwindgeschwindigkeit $(w)$ in Abängigkeit von der Schubspannungsgeschwindigkeit $\left(u_{*}\right)$ aus allen verfügbaren Nachtdaten des Untersuchungszeitraumes (Messhöhe: $48 \mathrm{~m}$ ). 
Wie äußert sich nächtliche Advektion am Standort Bariri? Zur Beantwortung dieser Frage erscheint es sinnvoll, die mittlere Vertikalwindgeschwindigkeit in Abhängigkeit zu den Austauschbedingungen $\left(u_{*}\right)$ zu betrachten. Mit Zunahme der atmosphärischen Stabilität und des Kaltluftabflusses dringt mehr und mehr Luft aus dem Kronenraum in den Stammraum ein (Entrainment). Die Abbildungen 3.16 (b) und 3.18 zeigen dieses Verhalten deutlich. Mit zunehmender Stabilität und dadurch abnehmender Schubspannungsgeschwindigkeit nimmt die Vertikalwindgeschwindigkeit immer negativere Werte an. Außerdem zeigt Abb. 3.18 in bemerkenswerter Weise, dass die Varianz von $w$ infolge dominierender Schichtungsverhältnisse selbst bei kleinster Anzahl an Observationen auf konstant niedrigerem Niveau bleibt. Damit zeigt der Standort Bariri die typischen Merkmale eines durch katabatische Schwereflüsse hervorgerufenen Konvergenzmusters $\left[\mathrm{ABC}^{+} 05\right.$, AHY03].

Da in der Nacht das $\mathrm{CO}_{2}$-Mischungsverhältnis über dem Bestand größer ist als im Bestand und der mittlere vertikale Wind Luft aus dem Bereich über dem Bestand in den Bestand transportiert, ergibt sich, dass weniger $\mathrm{CO}_{2}$ in die Luftsäule im Bestand eingebracht wird als zur Seite wegtransportiert wird. Das hat zur Folge, dass in der Nacht kontinuierlich $\mathrm{CO}_{2}$ aus einem gedachten Kontrollvolumen unterhalb des Sensors entweicht. Abbildung 3.16 (b) zeigt einen vertikalen Fluss von etwa $1.1 g(C) d^{-1}{ }^{7}$, der nicht mit der EK-Methode erfasst werden kann.

Ähnliche Strömungsverhältnisse zeigen z.B. auch die Standorte Browns River und Prince Albert [Lee98], Vielsalm [ABC ${ }^{+} 05, \mathrm{ACE}^{+}$03], Tharandt [FBV03] und Renon $\left[\mathrm{MCM}^{+} 05\right]$. Im ersten Fall fand Lee [Lee98] positive vertikale Advektion und postulierte, dass die horizontale Advektion Null sei. Die Einwände Finnigans aus [Fin99] wurden ausführlich in Abschn. 2.1.2.1 diskutiert. Anhand eines einfachen zweidimensionalen Modells $\left[\mathrm{BEF}^{+} 06, \mathrm{ABC}^{+} 05\right]$ lässt sich zeigen, dass Lees Annahme allein im Fall konstanter $\mathrm{CO}_{2}$-Quellstärke plausibel ist. Die Untersuchungen in Vielsam und Tharandt wiesen einen in Strömungsrichtung negativen horizontalen $\mathrm{CO}_{2^{-}}$Gradienten auf. Leider stehen in beiden Fällen flächendeckende Untersuchungen zur Quellstärkenverteilung noch nicht zur Verfügung (Aubinet 2006, persönl. Mitteilung). Das Modell von Aubinet stellt für die Untersuchungen am Standort Bariri einen großen Gewinn dar, da im Rahmen der Messkampagne zwar horizontale Advektionsmessungen nicht durchzuführen waren, Quellgebietsuntersuchungen aus Bodenkammermessungen jedoch flächendeckend vorlagen [Jin06]. In Tabelle 3.4 sind die wesentlichen Ergebnisse des Modells von Aubinet $\left[\mathrm{ABC}^{+} 05\right]$ zusammengefasst. Die Bodenhaubenuntersunchun-

\footnotetext{
${ }^{7}$ Relevant ist vor allem der Bereich $u_{*} \leq 0.4 \mathrm{~ms}^{-1}$, da in diesem Bereich die Anzahl an Observationen dominiert (vergl. Häufigkeitsverteilung Abb. 3.16 a).
} 
Tagesgang der $\mathrm{CO}_{2}$ Konzentration

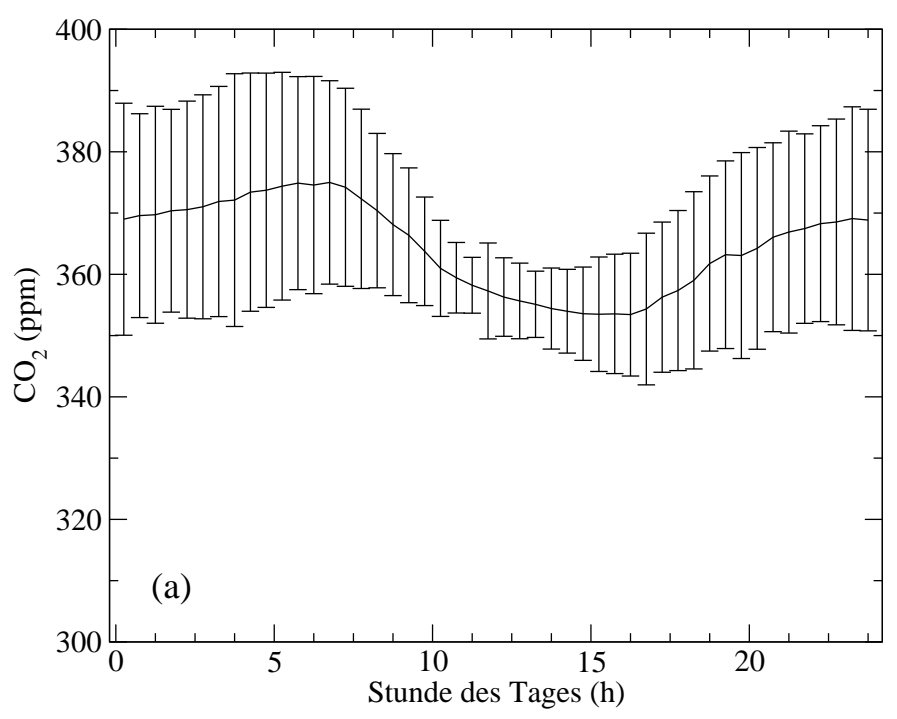

Tagesgang des turbulenten $\mathrm{CO}_{2}$-Flusses（ges. Datensatz)

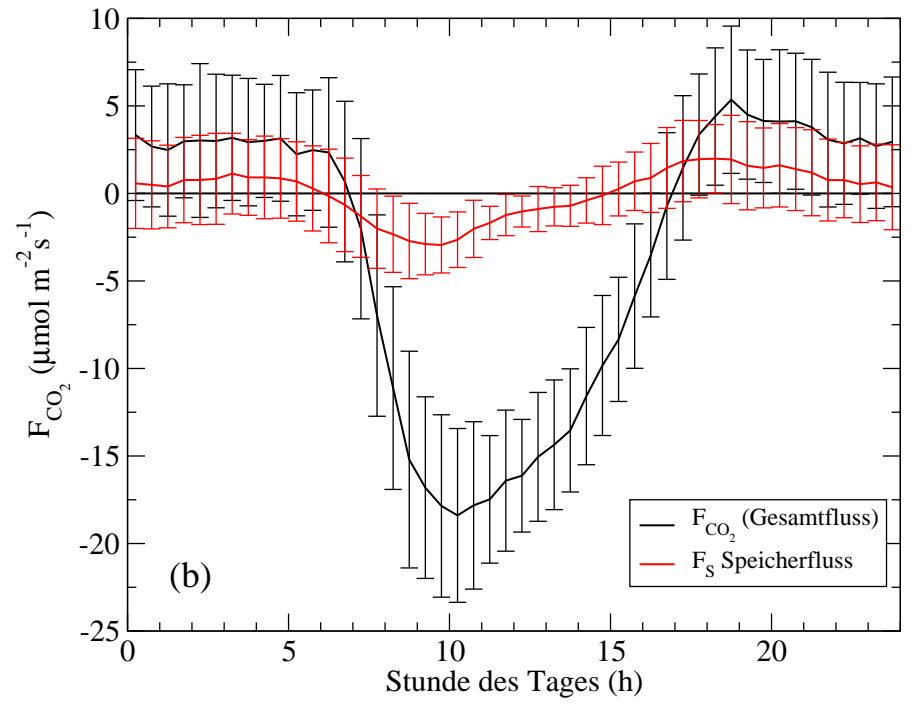

Abbildung 3.17: Mittlerer Tagesgang (a) der $\mathrm{CO}_{2}$-Konzentration und (b) des $C_{2}$-Gesamtflusses $F_{C}+F_{S}$ sowie des Speicherflusses $F_{S}$ separat aus allen verfügbaren Daten. 
Tabelle 3.4: Klassifikation der verschiedenen Advektionsmuster in Beziehung zur Quellstärkenverteilung und Massenflusscharakteristik Ref.: [ABC $\left.{ }^{+} 05\right]$. HA: horizontale Advektion, VA: vertikale Advektion

\begin{tabular}{lrrr}
\hline & Konvergenz & Konst. Massenfluss & Divergenz \\
\hline $\begin{array}{l}\text { Abnehmende Quellstärken- } \\
\text { intensität }\end{array}$ & $\mathrm{VA}>0$ & $\mathrm{VA}=0$ & $\mathrm{VA}<0$ \\
& $\mathrm{HA}<0$ & $\mathrm{HA}>0$ & $\mathrm{HA}>0$ \\
& $\mathrm{HA}|<| \mathrm{VA} \mid$ & $\mathrm{VA}=0$ & $\mathrm{HA}|>| \mathrm{VA} \mid$ \\
$\begin{array}{l}\text { Konstante Quellstärken- } \\
\text { intensität }\end{array}$ & $\mathrm{HA}=0$ & $\mathrm{HA}>0$ & $\mathrm{HA}>0$ \\
& $\mathrm{VA}>0$ & $\mathrm{VA}=0$ & $\mathrm{HA}|>| \mathrm{VA} \mid$ \\
$\begin{array}{l}\text { Zunehmende Quellstärken- } \\
\text { intensität }\end{array}$ & $\mathrm{HA}>0$ & $\mathrm{HA}>0$ & $\mathrm{HA}>0$ \\
& & & $|\mathrm{HA}|>|\mathrm{VA}|$ \\
\hline
\end{tabular}

gen am Standort Bariri ergaben einen moderaten Anstieg der Quellstärke von $2.09 \mu \mathrm{mol} \mathrm{m} \mathrm{m}^{-2} \mathrm{~s}^{-1}$ auf $120 \mathrm{~m}$ in Strömungsrichtung [Jin06]. Aus der hangabwärts gerichteten Strömung und dem gleichgerichteten Konzentrationsgradienten ergäbe sich somit ein positiver Fluss (siehe Tab. 3.4). D.h., sowohl der vertikale als auch der horizontale advektive $\mathrm{CO}_{2}$-Fluss wirkt in dieselbe Richtung. Beide Flüsse führen zu einer Abreicherung vom Kohlendioxid im betrachteten Kontrollvolumen $\left[\mathrm{MCM}^{+} 05\right]$. Dies ist insofern ein großer Informationsgewinn, als dass uns Flüsse mit entgegengesetztem Vorzeichen vor die Problematik gestellt hätten, zu entscheiden, welcher Fluss der dominierende wäre. Ein Sachverhalt, der insbesondere in Bezug auf den horizontalen Fluss schwer zu beantworten ist, da direkte Messungen zur Zeit der Messkampagne nicht vorgesehen waren ${ }^{8}$.

Mithin erscheint eine quantitative Abschätzung des horizontalen advektiven Flusses zu diesem Zeitpunkt nicht möglich. Für die vertikale Advektion ergibt sich nach Gleichung 2.10 (Term $I V$ ) im Mittel ein täglicher Wert von $0.56 \pm 0.28 g(C) m^{-2}$. Das würde einer Jahressumme von $204 \pm 103 g(C) m^{-2}$ entsprechen. Dieser Wert wird etwas später im Kontext der Fehleranalyse zu diskutieren sein.

Zusammenfassend bleibt festzustellen: Mit jährlichen $204 g(C) \mathrm{m}^{-2}$ scheint ein beträchtlicher Anteil $\mathrm{CO}_{2}$ am Sensor vorbei advektiert zu werden. Die Gesamtökosystematmung scheint von dem EK-System also nicht komplett

\footnotetext{
${ }^{8}$ Messungen zur Berechnung der horizontalen Advektion werden in der laufenden Phase des SFB 552 angeschlossen, sodass in naher Zukunft mit ersten Ergebnissen zu rechnen ist.
} 

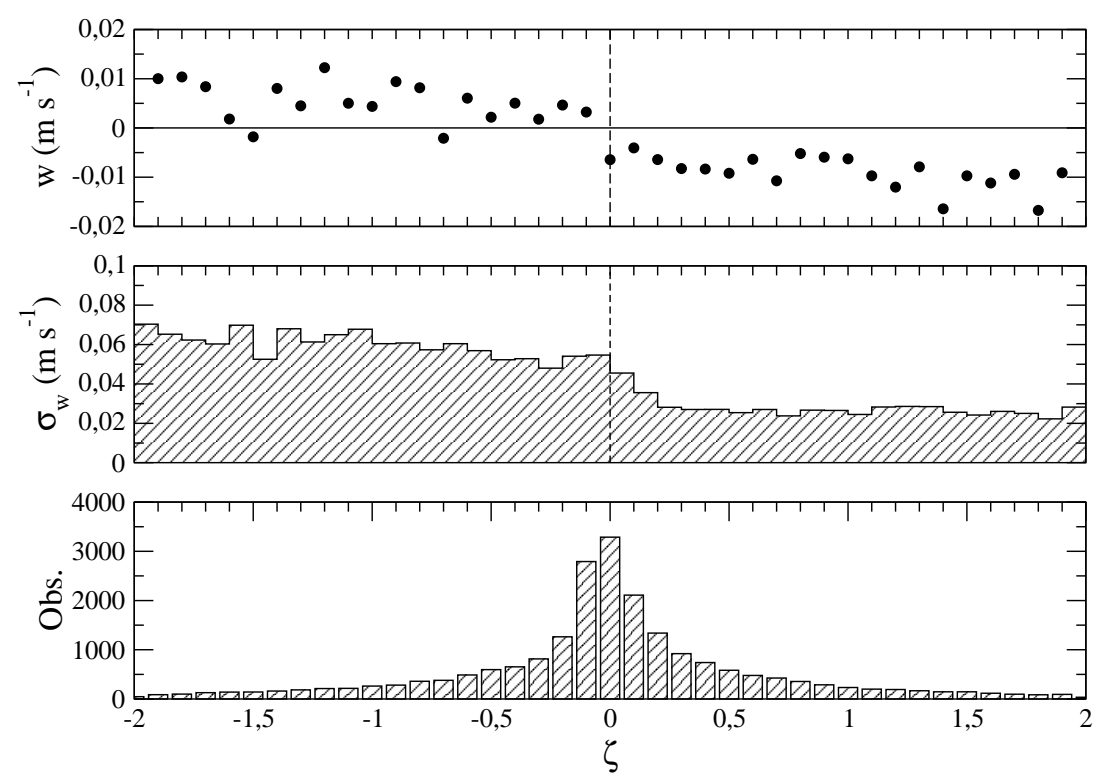

Abbildung 3.18: Abhängigkeit der mittleren Vertikalwindgeschwindigkeit $w$ und ihrer Varianz $\sigma_{w}$ von dem Stabilitätparameter $\varsigma$.

erfasst zu werden. Und ein weiterer horizontaler Flussanteil könnte diesen Effekt nach derzeitigem Wissensstand weiter forcieren. Die akkumulierte Netto - $\mathrm{CO}_{2}$-Aufnahme aus der Berechnung der turbulenten Flüsse würde demnach sehr wahrscheinlich systematisch überschätzt werden. Aus diesem Grunde kommt dem $u_{*}$-Filterkriterium eine wesentliche Bedeutung zu. Denn, wie gezeigt, nimmt die vertikale Advektion mit der mittleren Strömung und zunehmender Turbulenz ab.

\subsubsection{Berechnung advektiver $\mathrm{CO}_{2}$-Flüsse mittels eines $E$ - $\omega$ Modells für neutrale Schichtung}

Im vorangegangenen Abschnitt wurde ausführlich auf die speziellen Strömungsverhältnisse in der Nacht eingegangen. Es wurde herausgearbeitet, dass die EK-Methode am Standort Bariri unter bestimmten meteorologischen Voraussetzungen lediglich unter Vorbehalt anwendbar ist. Komplexe Strömun- 
gen entstehen aber auch ohne den Einfluss katabatischer Schwereflüsse, allein durch die orografischen Geländeverhältnisse bedingt.

Aus diesem Grunde wird im Folgenden der Versuch unternommen, den störenden Einfluss der Topografie auf die $\mathrm{CO}_{2}$-Flussmessungen unter neutralen atmosphärischen Schichtungsbedingungen zu quantifizieren. Dazu wird das in Abschn. 2.4 eingeführte 3d-Grenzschichtmodell verwendet [SL04, SMHL02]. Die Untersuchung der verschiedenen Windrichtungen findet in $30^{\circ}$ Schritten $\left(0-330^{\circ}\right)$ statt. Den Ausführungen aus Abschnitt 3.3.2 folgend gilt den drei Hauptwindrichtungen dabei besonderes Augenmerk.

Für jede der Windrichtungen wird das $3 d$-Strömungsregime zum einen für flaches, horizontal homogenes und zum anderen für real komplexes Geländerelief gerechnet. Anschließend wird das Verhältnis aus reliefbeeinflusstem zu reliefunbeeinflusstem Kohlendioxidfluss gebildet. Die so entstandenen Abweichungen bieten die Möglichkeit, den störenden Einfluss des heterogenen Geländes auf den vertikalen $\mathrm{CO}_{2}$-Fluss zu quantifizieren.

\subsubsection{Modellierung des vertikalen $\mathrm{CO}_{2}$-Flusses unter besonderer Berücksichtigung der Quellen und Senken}

Der vertikale Kohlendioxidfluss über dem Vegetationsbestand setzt sich aus respiratorischen und assimilatorischen Flüssen zusammen. Daher verspricht die strömungsphysikalische Analyse, wenn zunächst die einzelnen Flüsse separat und später die Flüsse in ihrem Zusammenwirken betrachtet werden, einen großen Erkenntnisgewinn. Derartige Untersuchungen wurden bereits in einer $2 d$ Version des Modells erfolgreich durchgeführt [SL04, SRV04, SPGV05]; mit dieser Studie werden nun erstmals $3 d$-Untersuchungen vorgestellt. Die Darstellung findet zunächst in Form von vertikalen NS- respektive WO-Schnitten durch den Turmstandort und weiter unten im horizontalen Schnitt in Messhöhe statt. Die schwarzen Pfeile auf den Abbildungen stellen die $x$ - respektive $y$-Komponente der vorgegebenen Windrichtung dar. Beim lateralen wie beim horizontalen Schnitt ist der Turmstandort bei $x$ (E-W Richtung) respektive $y$ (N-S Richtung) $=0$ gelegen ${ }^{9}$. Das EK-Messsystem ist in 48 Meter Höhe $20.7 m$ über dem Bestand lokalisiert. Bei den nachfolgenden Untersuchungen wurden die betrachteten Flüsse daher mit dem reliefunbeeinflussten Flusswert $\left(\mathrm{FCO}_{2}(-\infty, 48)\right)$ dieser Höhe normalisiert $(x, y=-\infty$ symbolisiert $\mathrm{CO}_{2}$-Flüsse, wie sie weit im Luv über ideal-flachem Gelände gemessen werden würden).

\footnotetext{
${ }^{9}$ Ausdehnung des modellierten Untersuchungsgebietes: $2 x 2 x 2.5 \mathrm{~km}$, Gitterzellgröße: $50 x 50 m$ (horizontal) und 3-100m (vertikal) (vergl. Abschn. 2.4).
} 
Für die Berechnungen wurde für die Bodenrespiration ${ }^{10}$ vereinfachend eine konstante Quellgebietsstärke von $5 \mu \mathrm{mol} \mathrm{m} \mathrm{m}^{-2} \mathrm{~s}^{-1}$ abgeschätzt. Die Stärke der fotosynthetischen Kohlendioxidaufnahme des Bestandes wurde in Abhängigkeit zur Höhe proportional zur $L A D$ Verteilung parametrisiert.
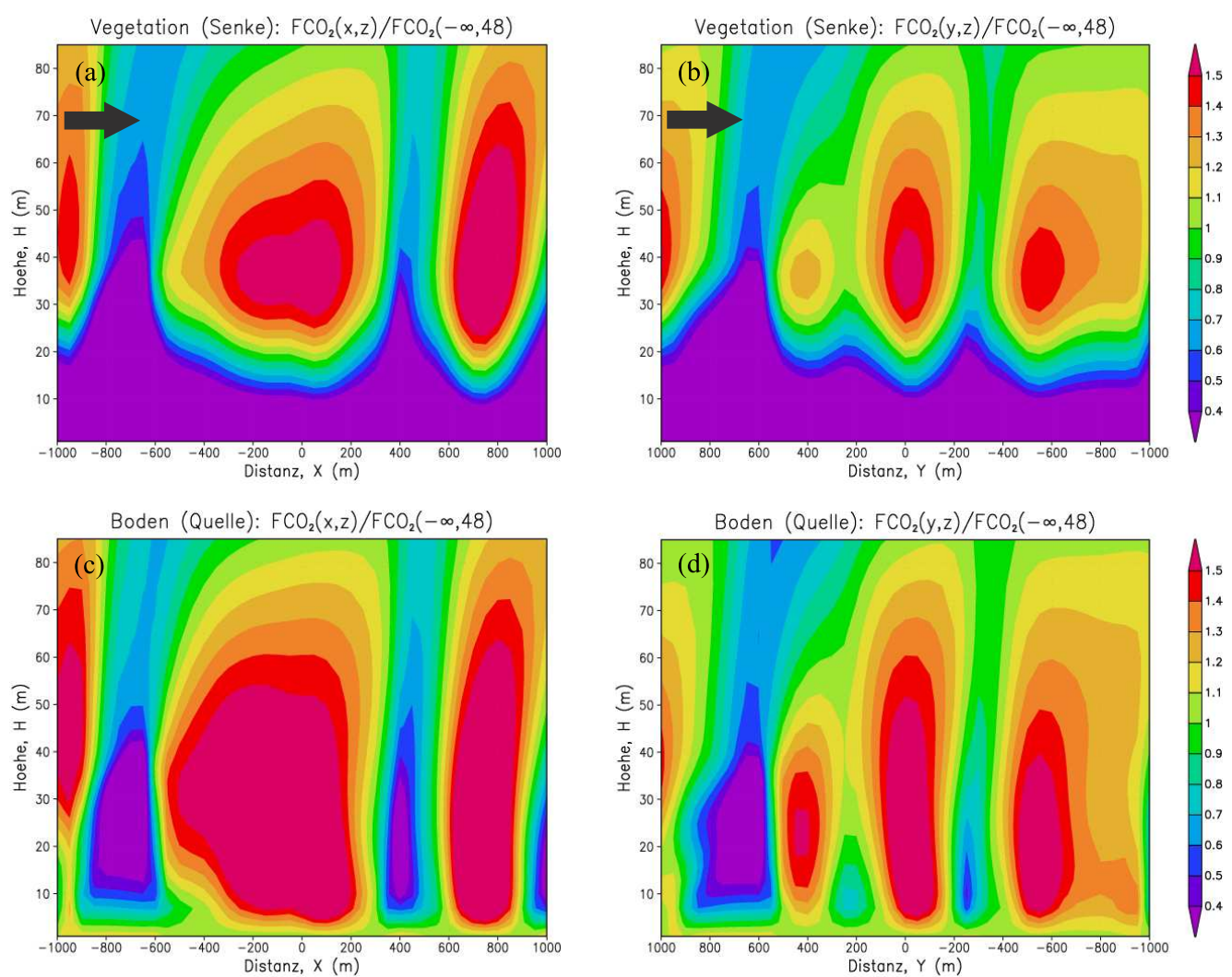

Abbildung 3.19: Normalisierte $(\mathrm{a}, \mathrm{b})$ assimilatorische und $(\mathrm{c}, \mathrm{d})$ respiratorische $\mathrm{CO}_{2}$-Flüsse $\mathrm{FCO}_{2}\left(x_{1,2}, 48\right) / \mathrm{FCO}_{2}(-\infty, 48)$ im vertikalen Schnitt durch das $3 d$-Strömungsregime bei fest vorgegebenem geostrophischen Wind aus $300^{\circ}$ und neutraler atmosphärischer Schichtung. In $x$-Richtung verläuft der Schnitt von $\mathrm{W}$ nach $\mathrm{O}$ und in $y$-Richtung von $\mathrm{N}$ nach $\mathrm{S} ;\left(x_{1,2}=0\right)$ bezeichnet den Turmstandort und die Messhöhe beträgt $z_{r}=48 \mathrm{~m}$. Als Vorgabe wurde aus den Messungen für die Bodenrespiration ein Wert von $5 \mu \mathrm{mol} \mathrm{m} \mathrm{m}^{-2} \mathrm{~s}^{-1}$ übernommen. Die $\mathrm{CO}_{2}$-Senkenstärke der Vegetation verläuft proportional zur vertikalen $L A D$ Verteilung.

Durch Vergleich der Abbildungen 3.19 (a,b) mit den Abbildungen (c,d) las-

\footnotetext{
${ }^{10}$ Respiration innerhalb des Bestandes (Blatt- und Holzatmung) wurde vernachlässigt.
} 

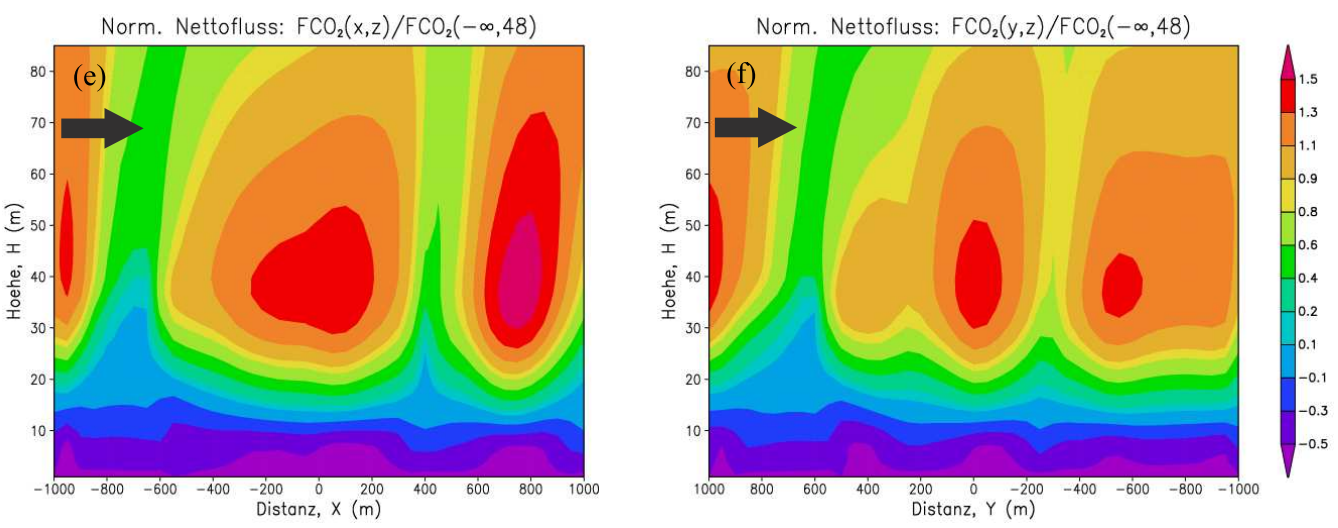

Abbildung 3.20: (Wie Abb. 3.19 a-d) Dargestellt ist der normalisierte vertikale Netto- $\mathrm{CO}_{2}$-Fluss in einem dem Relief folgenden Koordinatensystem.

sen sich die Unterschiede zwischen reliefbeeinflussten assimilatorischen und reliefbeeinflussten respiratorischen $\mathrm{CO}_{2}$-Flüssen herausarbeiten. Bezogen auf die Mess-höhe bedeuten rot-gelb kolorierte Areale eine Überschätzung und blau-violett farbene Bereiche eine Unterschätzung des Flusses im Vergleich zum homogenen Fall. Erwartungsgemäß ähneln sich die Flüsse, ob sie nun von Quellen oder Senken herrühren. Offensichtlich entwickeln sich die reliefinduzierten Störungen ${ }^{11}$ (in $48 m$ Höhe) um so stärker, je näher die Quelle dem Boden ist. Durch die Verwendung eines der Orografie folgenden Koordinatensystems wird besonders deutlich, dass die Störungen der Abb. (a,b) verglichen mit $(\mathrm{c}, \mathrm{d})$ oberhalb der Messungen etwa 5-10m früher abklingen. Dies deutet auch darauf hin, das Nachtflüsse auch abseits der Problematik katabatischer Schwereflüsse stärker fehlerbehaftet sind als das bei Tageslicht (wenn der fotosynthetische Fluss dominiert) der Fall ist [SRV04].

Auf den Abbildungen $3.20(\mathrm{e}, \mathrm{f})$ ist der Gesamtfluss - bestehend aus den beiden gegenläufig wirkenden Einzelflüssen - dargestellt. Die Störungen scheinen in der Summe etwas weniger ausgeprägt zu sein. Zwischen 10-20m innerhalb der Vegetation wechselt die Richtung der Flüsse. Nahe dem Waldboden beginnt der respiratorische zu dominieren.

\footnotetext{
${ }^{11}$ Als Störungen werden in diesem Kontext die Abweichungen vom $\mathrm{CO}_{2}$-Fluss über homogenem Relief bezeichnet.
} 


\subsubsection{Modellierung der vertikalen $\mathrm{CO}_{2}$-Flüsse in Abhängigkeit von der Windrichtung}

In diesem Abschnitt sollen nun die Hauptwindrichtungen detaillierter analysiert werden. Die Abbildungen 3.21 (a-f) entsprechen prinzipiell den Darstellungen 3.19 und 3.20, jedoch wird nun die Horizontalvariation der vertikalen Kohlendioxidflüsse in Abhängigkeit vom Bodenrelief veranschaulicht. Auf den Abbildungen 3.21 (a,c,e) ist jeweils in Farbe der normalisierte Fluss $\mathrm{FCO}_{2}(x, y, 48) / \mathrm{FCO}_{2}(-\infty,-\infty, 48)$ dargestellt. Außerdem ist die Orografie in Form von Konturlinien (Angaben in Metern) mitangegeben worden. Ebenso auf den Grafiken 3.21 (b,d,f); dort ist das Relief koloriert dargestellt. Und um den Einfluss des Geländes auf das Strömungsregime noch besser beurteilen zu können, sind zur Visualisierung Strömungslinien angegeben. Die verschiedenen Graustufen entsprechen dabei unterschiedlichen Windgeschwindigkeiten.

Die zeitlich am geringsten vertretende Hauptwindrichtung stellen Ostwinde $\left(90^{\circ}\right)$ dar (vergl. Abb. 3.1). Abbildung 3.21 (b) zeigt, dass der Wind, aus Ost kommend, bedingt durch die leichte Bodensenke (blau-violett gekennzeichnet), zunächst leicht entschleunigt und dann entlang der leichten Ausbuchtung geführt wird (Kanalisierungseffekt). Gleichzeitig beschleunigt der Wind, sobald eine Anhöhe genommen werden muss (erkennbar am Farbverlauf der Strömungslinien von dunkel nach hellgrau). Dass die höchsten Windgeschwindigkeiten beim Überströmen einer Reliefwelle kurz vor Erreichen einer Bergspitze auftreten, ist indes ein bekanntes Phänomen, welches beispielsweise sehr ausführlich in [FB95] beschrieben wird. Auch der Turmstandort $(x, y)=(0,0)$ ist innerhalb so eines Bereichs beschleunigter Strömung gelegen. Dieses Wechselspiel konvergenter und divergenter respektive schnellerer und langsamerer Strömungen führt zu einem norm. $\mathrm{CO}_{2}$-Fluss am Ort der Messungen von etwa 0.65. D.h., der vertikale Fluss wird - verglichen mit homogenen Verhältnissen - ziemlich stark unterschätzt.

Winde aus WSW wurden im vorangegangenen Abschnitt ausführlich im Nachtflusskontext besprochen. Im Fall der Abb. 3.21 (c,d) strömt der Wind im Wesentlichen von dem massiveren Höhenzug am unteren linken Bildrand hinab. Die Höhendifferenz zum Plateau, auf dem der Turm gelegen ist, beträgt etwa 150 - 200 Meter. Abweichungen in der Windrichtung entstehen hauptsächlich dort, wo die Anhöhe seitwärts umströmt wird. So wird die Strömung z.B. südwestsüdlich vom Turm in einem kleinen Arial stark entschleunigt (Abb. d); teilweise kommt es dabei zum Richtungswechsel der Strömung. In diesen Zonen gesteigerter Turbulenz herrschen entsprechend der Gradienten-Diffusionsapproximation stark veränderte Austauschbedin- 

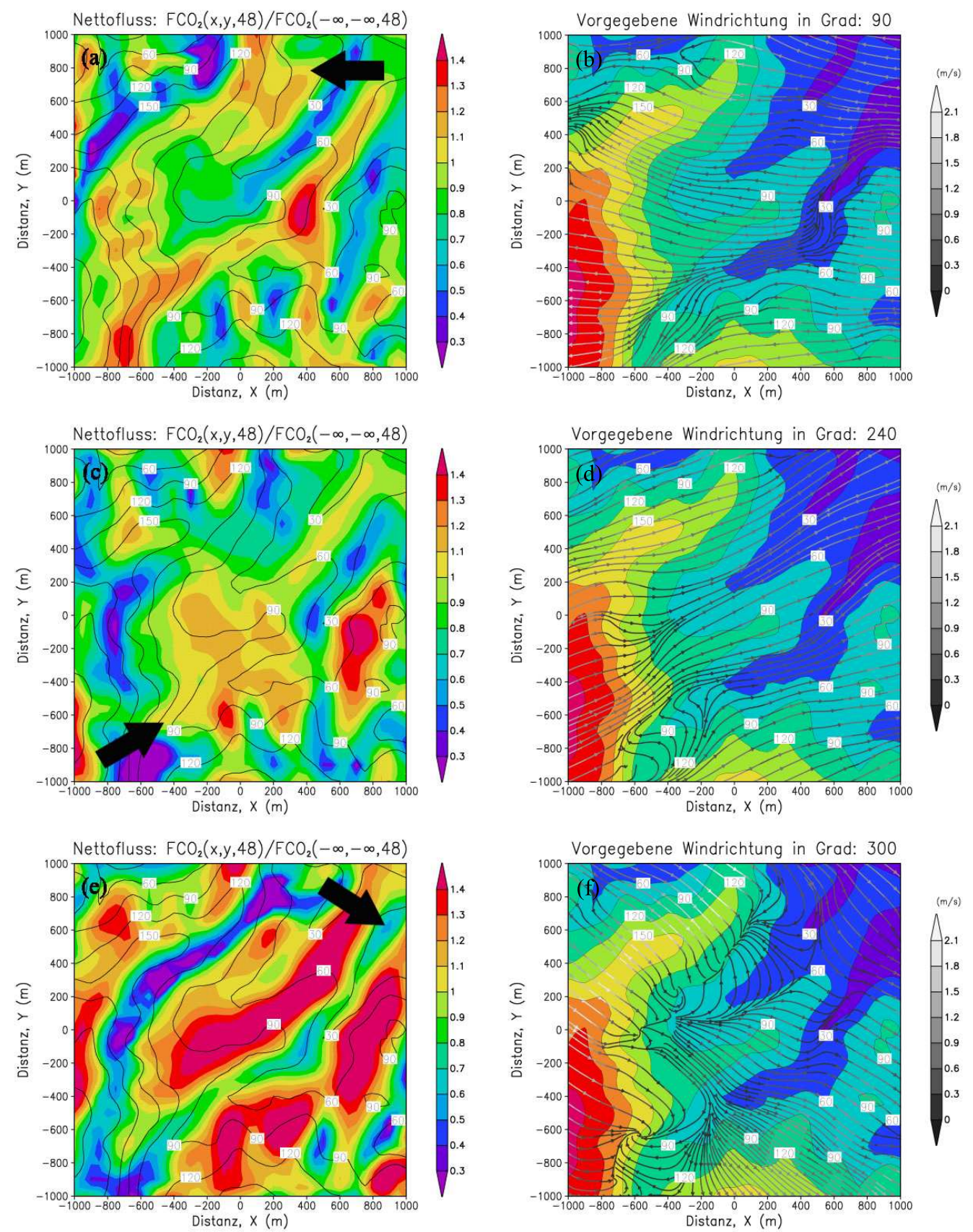

Abbildung 3.21: Modellierte vertikale $\mathrm{CO}_{2}$-Flüsse (neutrale atmosphärische Schichtung) und Strömungslinien über dem Untersuchungsgebiet. Dargestellt sind die normalisierten Vertikalflüsse (a,c,e) als auch die Strömungslinien (b,d,f) $48 m$ über dem Relief (für die Abb. b,d und $\mathrm{f}$ ist das Relief farbig unterlegt) im horizontalen Schnitt. Die vorgegebenen Windrichtungen (Pfeil) entsprechen den Hauptwindrichtungen und betragen in meteorologischer zählweise $90^{\circ}(\mathrm{a}, \mathrm{b}), 240^{\circ}(\mathrm{c}, \mathrm{d})$ und $300^{\circ}(\mathrm{e}, \mathrm{f})$. $(\mathrm{X}, \mathrm{Y})=(0,0)$ gibt den Turmstandort an. 
gungen - erkennbar an der blau-violetten Färbung (Abb. 3.21 c). Der Turmstandort ist von diesen Störungen allerdings wenig betroffen; der normalisierte Fluss liegt dort zwischen 0.9 und 1.

Ein Beispiel für ein stärker topografie-beeinflusstes Strömungsregime stellen die Abbildungen 3.21 (e,f) dar. In diesem Fall wird die Strömung über ein von der Orografie vorgegebenes Wellenprofil gezwungen. Eine seitliche Umströmung eines Hindernisses ist nur begrenzt möglich, da die Störung quer zur Ausbreitungsrichtung stark ausgedehnt ist. Die Situation ist sehr gut mit idealisierten $2 d$-Simulationen vergleichbar, bei denen ein sinusförmiger Wellenberg vorgegeben wurde [FB95, SRV04, LL04]; auf dem Weg die Anhöhe hinauf wird die Strömung leicht komprimiert, sodass die Strömung beschleunigt wird (Abb.3.21 f, hell-grau bis weiss gefärbte Strömungslinien). Ist die Topografie etwas steiler ausgeprägt, wie z.B. nordwestl. von Turm, kann es leeseitig zu Strömungsablösungen (separation bubble) kommen. Die Strömungsgeschwindigkeit nimmt stark ab, und es kann sogar zu einer kompletten Umkehr der Strömungsrichtung kommen (reversed flow). Derartige Verhältnisse führen zu stark orografieabhängigen Skalarflussvariationen; deutlich zu erkennen an dem Wechselspiel rot-magenta und blau-violett farbener Bereiche quer zur Windrichtung (Abb. 3.21 e). Für den Turmstandort ergibt sich ein vergleichsweise hoher Wert des norm. Kohlendioxidflusses von 1.40-1.45 (vergl. Abb. 3.21 e).

Um außer zu den Netto-Kohlendioxidflüssen auch vergleichende Aussagen über Abweichungen in den assimilatorischen respektive respiratorischen Flüssen machen zu können, wurde der normalisierte Netto- $\mathrm{CO}_{2}$-Fluss noch einmal zusammen mit seinen Komponentenflüssen dargestellt. Die Darstellungen 3.22 (a-d) und 3.23 (e,f) entsprechen im Prinzip den Abbildungen 3.21 (a,c,e); jedoch werden die normierten Flüsse lediglich in NS respektive WO Richtung mit dem Turmstandort als Mittelpunkt $(x, y)=0$ betrachtet. Bereits die Flüsse im vertikalen Schnitt (vergl. Abb.3.19 a-d und 3.20 e,f) ließen vermuten, dass die normalisierten Bodenflüsse in 48 Meter Höhe in der Regel stärker von eins abweichen als die assimilatorischen Flüsse. In dieser Hinsicht zeigen sowohl die $3 d$-Untersuchungen hier, als auch die $2 d$-Untersuchungen in [SL04] ähnliche Ergebnisse. Dies ist vor allem deswegen bemerkenswert, weil in der Nacht, wenn keine Fotosynthese stattfindet, allein respiratorische Flüsse wirksam sind. Wie bereits erwähnt, folgert Sogachev in diesem Sinne [SL04], dass nicht nur katabatische Kaltluftabflüsse, sondern auch die immer auftretende, orografiebedingte Beeinflussung der Strömung bei Nacht ein größeres Problem darstellen könnte. Dabei ist jedoch zu beachten, dass neutrale Schichtungsverhältnisse, für die das Modell in der vorliegenden Form konzipiert ist, nicht selbstverständlich auf stabile Schichtungsverhältnisse übertragen werden können. 

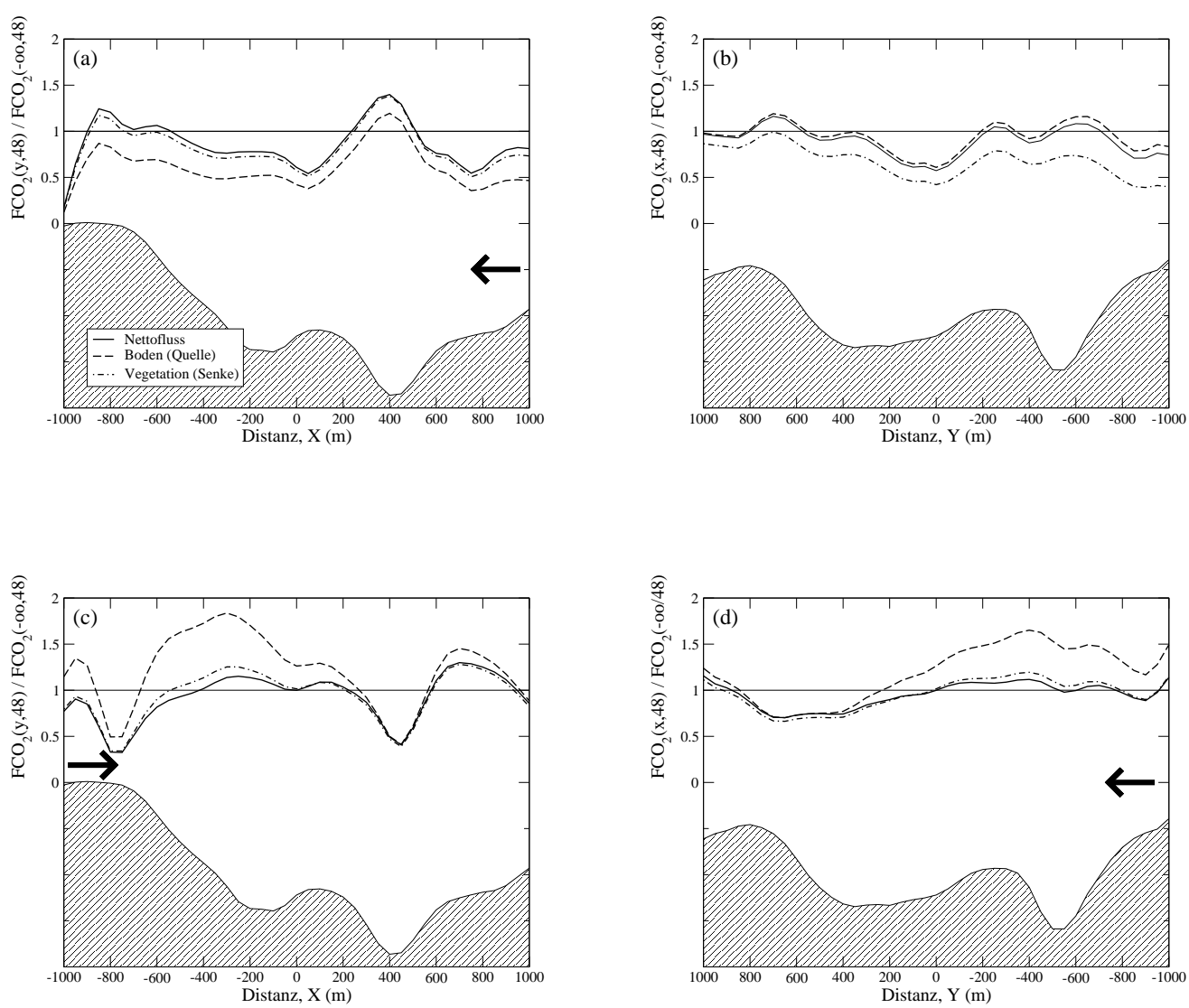

Abbildung 3.22: Wie Abb. 3.19, jedoch werden lediglich die normalisierten Flüsse in Messhöhe $(48 \mathrm{~m})$ dargestellt. Zusätzlich zum normalisierten $\mathrm{CO}_{2}{ }^{-}$ Nettofluss sind zur Veranschaulichung die normalisierten respiratorischen und assimilatorischen Flüsse angegeben. Die modellierten Windrichtungen entsprechen den gemessenen Hauptwindrichtungen: $90^{\circ}(\mathrm{a}, \mathrm{b}), 240^{\circ}(\mathrm{c}, \mathrm{d})$. $(\mathrm{X}, \mathrm{Y})=(0,0)$ gibt den Turmstandort an. Die Pfeile geben die $x$ - (WO) respektive die $y$ - Komponente (NS) der betrachteten Hauptwindrichtung an.

Um etwas später - zur Berechnung des Nettoökosystemaustauschs - den topografiebedingten Gesamtfehler des $\mathrm{CO}_{2}$-Flusses abschätzen zu können, wurden neben den Hauptwindrichtungen auch die Fehler aller anderen Windrichtungen für den Ort des EK-Messsystems modelliert und berechnet. Abb. 3.24 zeigt den normalisierten $\mathrm{CO}_{2}$-Fluss in Abhängigkeit von der Windrichtung 

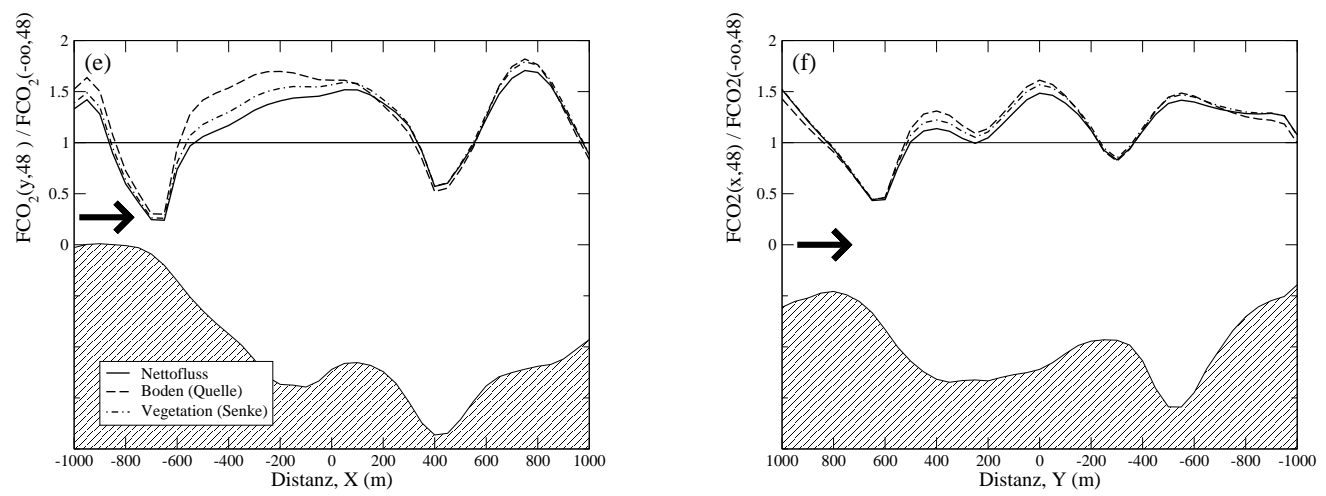

Abbildung 3.23: Wie Abb. 3.22 a-d. Dargestellt ist die geostrophische Windrichtung $300^{\circ}$.

und einer Auflösung von $30^{\circ}$. Als Resultat ergibt sich ein annähernd sinusförmiger Verlauf. Das Verhältnis $F C O_{2}(0,0,48) / F C O_{2}(-\infty,-\infty, 48)$ schlägt an den Punkten $45^{\circ}$ und $240^{\circ}$ um. Dieser Verlauf lässt sich plausibel anhand der charakteristischen Geländeverhältnisse erklären (vergl. Abb. 2.4). Offensichtlich stellt die langgestreckte Topografieerhebung, welche sich, ausgehend von der südwestlich gelegenen Anhöhe nordostwärts erstreckt und auf dessen Rücken der Turmstandort gelegen ist, das ausschlaggebende Kriterium dar. Strömt der Wind aus südöstlichen Richtungen $\left(45^{\circ}-240^{\circ}\right)$ über die Erhebung, ist das Verhältnis kleiner als eins, strömt er die Erhebung entlang ist es nahe eins und weht der Wind aus nordwestlichen Richtungen $\left(270^{\circ}\right.$ $40^{\circ}$ ), ergeben sich Werte größer als eins. Dabei scheint entscheidend, dass der Turmstandort in dem einen Fall (Wind aus NW) vor dem höchsten Punkt der Erhebung gelegen ist und im anderen Fall dementsprechend dahinter (im Lee) gelegen ist. Dies deckt sich mit den numerischen $2 d$-Experimenten über einer einzelnen sinusförmigen Bodenwölbung [SL04, RWCH92, $\mathrm{KFP}^{+}$06]. Auch dort nimmt der $\mathrm{CO}_{2}$-Fluss kurz nach überschreiten der Hügelspitze zunächst stark ab.

Aus Abb. 3.21 (e) wird deutlich, dass die zweite Hügelkette nordwestlich vom Turm, welche quasi parallel zur betrachteten Relieferhöhung verläuft, einen verstärkenden Effekt auf die skizzierten Verhältnisse bei Winden aus NW ausübt. Nach den vorangegangenen Untersuchungen stellt sich also heraus, dass die geländebedingte Beeinflussung der $\mathrm{CO}_{2}$-Flüsse am Standort Bariri nicht vernachlässigbar ist. 


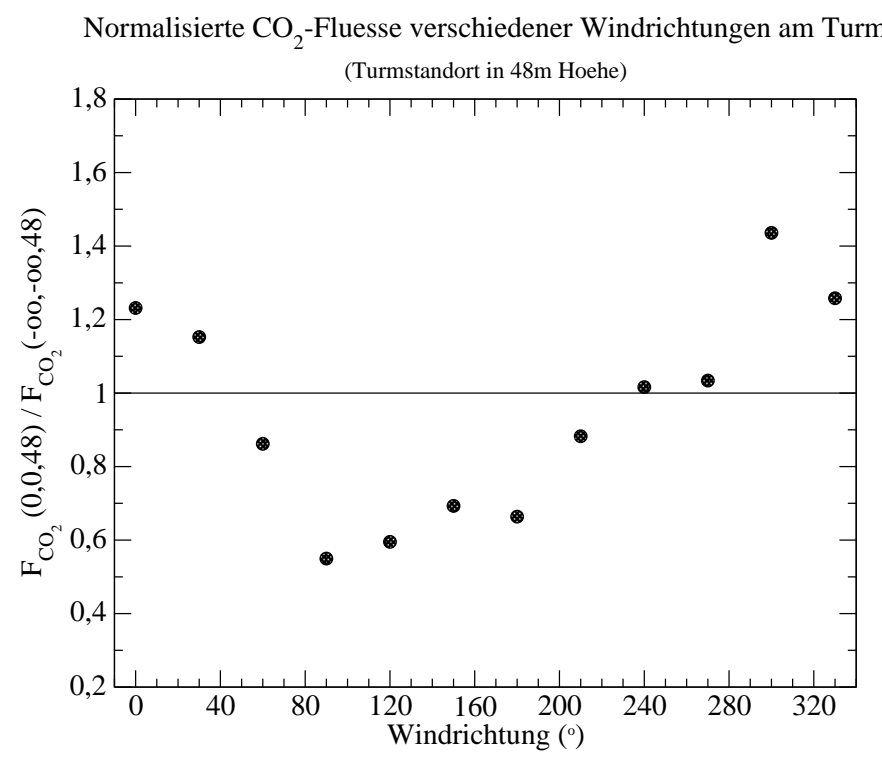

Abbildung 3.24: Erweiterung der Untersuchungen aus Abb. 3.21 (a,c,e) auf den gesamten Windrichtungsbereich. Angegeben sind die normalisierten Flüsse am Ort der EK-Messungen $48 m$ über Grund.

\subsection{Untersuchung der Kohlenstoffbilanz}

Im Folgenden Abschnitt des Ergebnisteils dieser Arbeit werden schließlich die Kohlenstoffflüsse des tropischen Hochlandregenwaldes in Indonesien bilanziert. Nach den vorangegangenen Untersuchungen soll dies zum einen nach den konventionellen Methoden - ohne Berücksichtigung advektiver Einflüsse - und zum anderen unter besonderer Berücksichtigung der advektiven Einflüsse geschehen.

Doch zuvor sind, um die turbulenten $\mathrm{CO}_{2}$-Flüsse über den gesamten Messzeitraum integrieren zu können, fehlende Messdaten mit Hilfe abgeleiteter Modelle zu ersetzen.

\subsubsection{Aufteilung des $\mathrm{CO}_{2}$-Flusses in seine Komponenten}

Wie bereits in Abschnitt 2.2 erläutert, ist zur Untersuchung der Kohlenstoffbilanz die Aufteilung des NEE (netto ecosystem exchange) in seine konkurrierenden Anteile von Bedeutung. Der respiratorische Anteil wird als Ökosystematmung (ecosystem respiration, RE) und der assimilatorische An- 
teil, welcher sich aus der Differenz zwischen $N E E$ und $R E$ ergibt, wird als Brutto-Fotosyntheserate (gross photosynthesis rate, GPR) bezeichnet. Abhängig vom Ökosystem werden $30-80 \%$ des brutto aufgenommenen Kohlendioxids durch Respiration wieder freigegeben [Reb03].

Wie aus vorangegangenen Bemerkungen bekannt ist, existieren Unterschiede zwischen Licht- und Dunkelatmung. Die aus der Michaelis-Menten Funktion abgeleitete Tagatmung $R_{\text {Tag }}$ (Abschn. 2.2.1.1) spiegelt auf der Bestandesebene die Atmung der Mikroorganismen, des Wurzelwerks, des Holzes sowie - im Unterschied zur Dunkelatmung - die Licht- oder Fotorespiration wider. Zwar nimmt auch die Lichtatmung mit zunehmenden Temperaturen zu; sie ist jedoch im Vergleich zur Dunkelatmung deutlich gehemmt [Jon92, AMGD00]. Daher wird zur Ermittlung der saisonalen Gesamtökosystematmung die Nachtatmung und die Tagatmung separat betrachtet.

\subsubsection{Darstellung der Nettoassimilation mit dem Modell von Michaelis-Menten}

Zur Darstellung der Nettoassimilation (Summe aus Fotosynthese und Respiration) wurden die Flüsse gegen die fotosynthetisch aktive Strahlung aufgetragen (vergl. Abschn. 2.2.1.1). Nach dem Modell von Michaelis-Menten wurde anschließend $\alpha$, die Quantenausbeute ( $\mathrm{mol} \mathrm{CO}_{2} \mathrm{~mol}^{-1}$ absorb.Photonen), $F_{\text {Sat }}$, die Brutto-Fotosyntheserate bei Lichtsättigung $\left(\mu \mathrm{mol} \mathrm{m} \mathrm{m}^{-2} \mathrm{~s}^{-1}\right)$ und $R_{\text {Tag }}$, die Ökosystematmung am Tage $\left(\mu m o l m^{-2} s^{-1}\right)$ ermittelt, indem die Funktion Glg. 2.28 an die Daten angefittet wurde. Doch zuvor wurde der gesamte Datensatz entsprechend den Ausführungen aus Abschn. 2.2.1.1 nach der Temperatur sowie nach dem Wolkenbedeckungsgrad klassifiziert. Durch diese Prozedur ließ sich die Streuung der Daten um 10 - 20\% reduzieren.

Durch Vergleich der verschiedenen Bedeckungsgrade (vergl. Abschn. 2.2.3) für feste Strahlungswerte fällt auf, dass im Fall der geschlossenen Wolkenschicht, die negativsten Flusswerte erreicht werden. Dies lässt sich leicht erklären: Bei starker Wolkenbedeckung ist beinahe die gesamte verfügbare Strahlung diffus, während bei klarem Himmel, die direkte Strahlung den weit überwiegenden Anteil der Strahlung ausmacht. Da diffuse Strahlung sehr viel tiefer in die Vegetation einzudringen vermag, tragen in verstärktem Maße auch die unteren Bestandesschichten zur Kohlendioxidaufnahme bei. Im Gegensatz dazu erreicht direkte Strahlung allein den sonnenbeschienenen Teil des Pflanzendaches, welcher nach unten exponentiell abnimmt.

Wie unterschiedlich die Nettoassimilation des Regenwaldbestandes in Abhängigkeit von den betrachteten Lichtverhältnissen und der Temperatur ausfällt, lässt sich noch anschaulicher anhand der Abb. 3.26 a-c darstellen. Deut- 

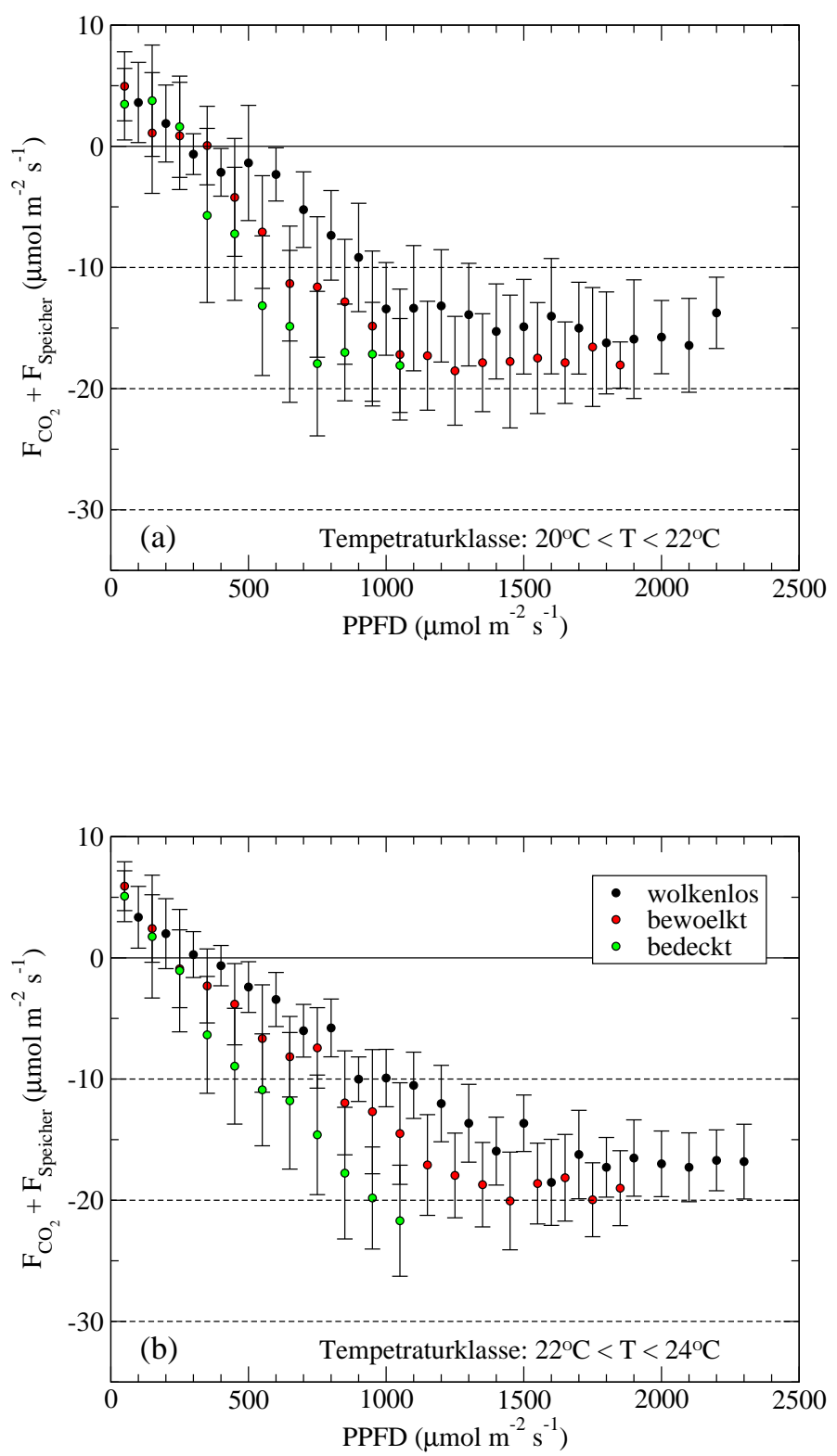

Abbildung 3.25: Darstellung des $C_{2}$-Flusses $\left(F_{C}+F_{S}\right)$ in Abhängigkeit von der fotosynthetisch aktiven Strahlung PPFD (photosynthetic photon flux density). Der Kurvenverlauf lässt sich durch die Michaelis-Menten Gleichung beschreiben. Nach Trennung des Datensatzes in Temperaturklassen von je $2^{\circ} \mathrm{C}$, wurde jede Temperaturklasse nochmals in drei Unterklassen nach Bewölkungsgrad (wolkenlos, bewölkt, bedeckt) unterteilt. Die Abbildungen zeigen beispielhaft die Temperaturklassen $20-22^{\circ} \mathrm{C}$ (a) und $22-24^{\circ} \mathrm{C}$ (b). 
Tabelle 3.5: Regressionsparameter der Lichtsättigungsfunktion nach Michaelis Menten. Aufgrund der vernachlässigbar kleinen saisonalen Schwankungen der $\mathrm{CO}_{2}$-Aufnahmeraten konnte die gesamte Messperiode berücksichtigt werden. Dargestellt sind Temperatur $T\left(^{\circ}\right)$, Bewölkungsgrad, maximale $\mathrm{CO}_{2}$-Aufnahme-rate $\mathrm{F}_{\mathrm{CO}_{2} \max }\left(\mu \mathrm{mol} \mathrm{m}^{-2} \mathrm{~s}^{-1}\right)$, Quantenausbeute $\alpha\left(\operatorname{mol}\left(\mathrm{CO}_{2}\right) \mathrm{mol}^{-1}\right.$ absorb. Photonen), Tagatmung $R_{\text {day }}\left(\mu \mathrm{mol} \mathrm{m} \mathrm{m}^{-2} \mathrm{~s}^{-1}\right)$, Lichtsättigung $F_{\text {Sat }}$ $\left(\mu \mathrm{mol} \mathrm{m} \mathrm{m}^{-2} \mathrm{~s}^{-1}\right)$ und das Bestimmtheitsmass $\left(R^{2}\right)$

\begin{tabular}{llrrrrrr}
\hline $\mathrm{T}$ & Bewölkungsgrad & $F_{\mathrm{CO}_{2} \max }$ & $\alpha$ & $R_{\text {day }}$ & $F_{\text {Sat }}$ & $R^{2}$ & Obs. \\
\hline \hline$<18$ & klar & -18.551 & -0.021 & 0.525 & -60.344 & 0.930 & 885 \\
& bewölkt $^{*}$ bedeckt $^{a}$ & -20.825 & -0.058 & 4.946 & -32.835 & 0.940 & 1246 \\
{$[18,20)$} & klar & -23.202 & -0.031 & 3.872 & -108.09 & 0.974 & 736 \\
& bewölkt & -17.869 & -0.068 & 4.853 & -44.733 & 0.992 & 1229 \\
& bedeckt & -23.487 & -0.072 & 5.589 & -36.786 & 0.970 & 1188 \\
{$[20,22)$} & klar & -20.997 & -0.068 & 6.853 & -44.733 & 0.992 & 854 \\
& bewölkt & -16.883 & -0.035 & 6.351 & -36.924 & 0.955 & 1411 \\
& bedeckt & -20.178 & -0.048 & 5.491 & -41.594 & 0.965 & 1361 \\
{$[22,24)$} & klar & -18.090 & -0.063 & 4.896 & -48.877 & 0.968 & 769 \\
& bewölkt & -16.413 & -0.0637 & 5.896 & -48.877 & 0.991 & 1695 \\
& bedeckt & -17.530 & -0.044 & 6.441 & -39.252 & 0.992 & 1210 \\
$>24$ & klar & -19.993 & -0.038 & 6.295 & -86.958 & 0.995 & 558 \\
& bewölkt & -11.425 & -0.016 & 3.075 & -20.883 & 0.776 & 794 \\
& & -18.937 & -0.024 & 6.456 & -48.162 & 0.974 & 395 \\
\hline
\end{tabular}

${ }^{a} F_{\text {Sat }}$ kann aufgrund der unzureichenden Datenlage an dieser Stelle nicht hinreichend genau bestimmt werden. Für die Gap-Filling Prozedur ergeben sich hierdurch allerdings keine weiteren Probleme, da die Michaelis Menten Funktion die $\mathrm{CO}_{2}$-Tagesaufnahmeraten für die betrachteten kleinen $P P F D$-Werte sehr gut widergibt. 
lich ist $\mathrm{zu}$ sehen, wie sich das Areal maximaler $\mathrm{CO}_{2}$ Aufnahme innerhalb einer gegebenen Temperaturklasse stetig weiter nach rechts verschiebt, sofern die Abbildungen nacheinander von links nach rechts, also von bedeckten (overcast) zu klaren (clear sky) Witterungsverhältnissen, betrachtet werden. Diese Ergebnisse sind auch bezüglich der Modellierungen zum Klimawandel von großem Interesse. Sollte die Wolkenbildung infolge der Klimaerwärmung forciert werden, würde auch der Anteil an diffuser Strahlung ansteigen und somit vermutlich auch die globale $\mathrm{CO}_{2}$-Aufnahme. Die Frage ist, wie groß die Bedeutung dieses Rückkopplungsprozesses als einer unter vielen tatsächlich ist.
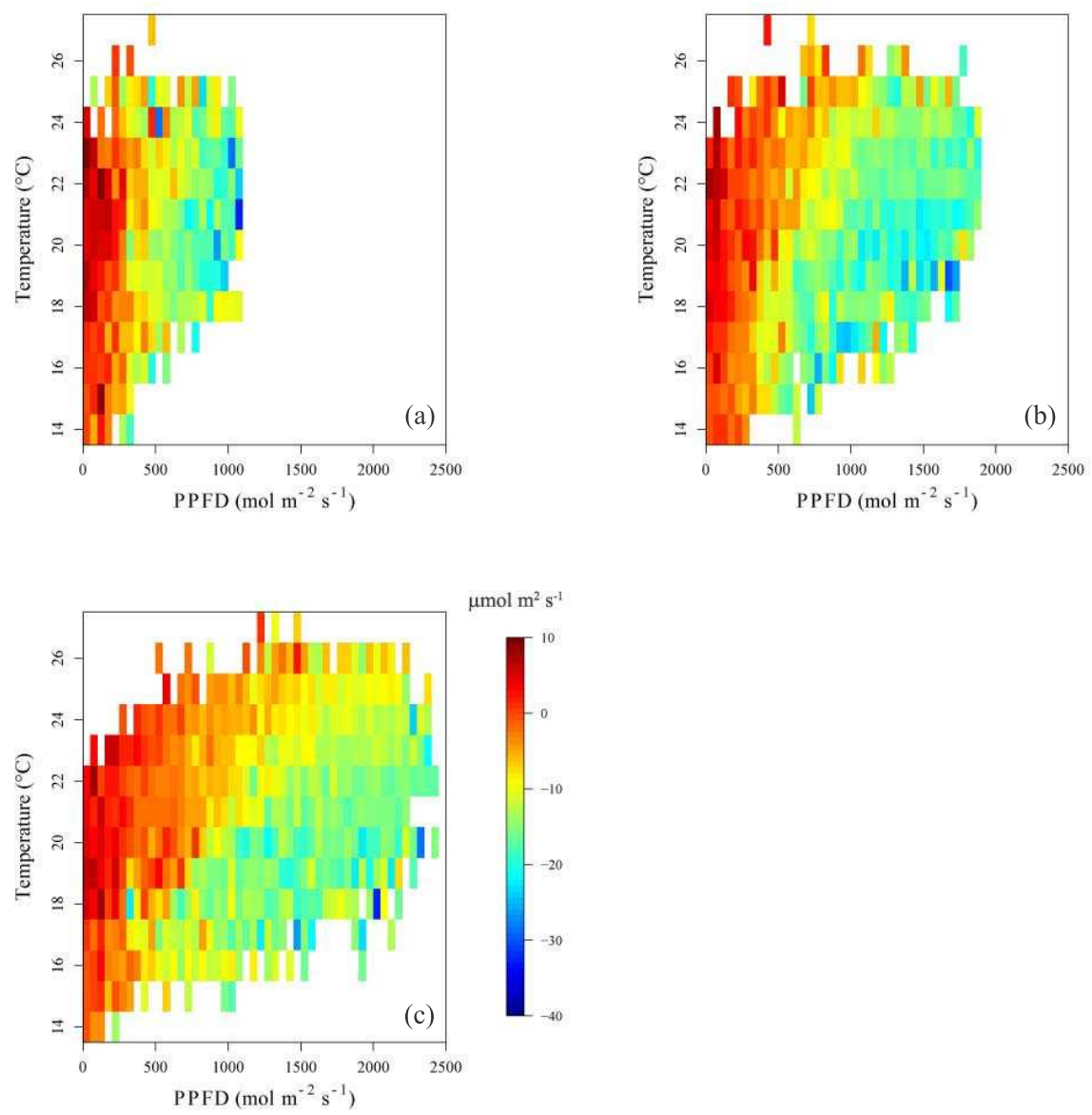

Abbildung 3.26: Temperatur- und Strahlungsantwort des NEE unter bedeckten (a), bewölkten (b) und klaren (c) Himmelsbedingungen. (Einheit: umol $\left.\mathrm{m}^{-2} \mathrm{~s}^{-1}\right)$ 
Abbildung 3.26 (a) zeigt z.B. auch, dass die $\mathrm{CO}_{2}$-Aufnahme bei diffusen Lichtverhältnissen etwas kontinuierlicher über den gesamten Temperaturbereich stattfindet. Auch bei höheren Temperaturen in Verbindung mit moderaten Strahlungswerten, nimmt der Regenwald noch in beachtlichem Ausmaß (15-20 $\left.\mu \mathrm{mol} \mathrm{m} \mathrm{m}^{-2}\right) \mathrm{CO}_{2}$ auf.
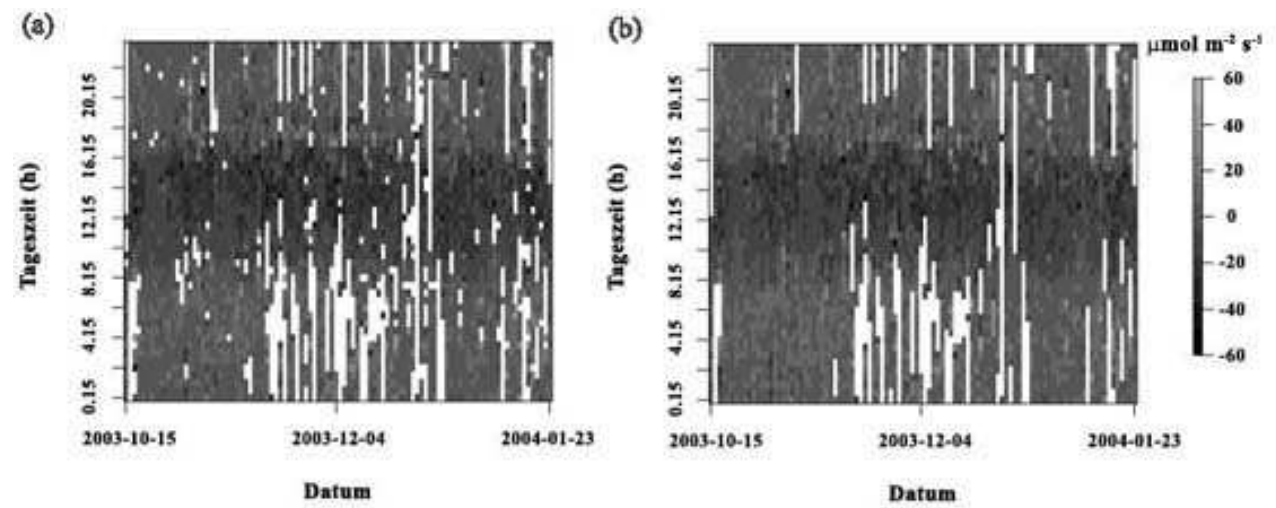

Abbildung 3.27: Gapfilling Prozedur: Fehlstellen $\leq 1.5 h$ wurden linear interpoliert. Grössere Lücken wurden nach Temperatur und Bewölkungsgrad mittels der Lichtsättigungskurven geschlossen.

Vor dem Durchführen der in Abschnitt 2.2.3 beschriebenen Prozedur zur Schließung der Datenlücken wurden zunächst Lücken $\leq 1.5 \mathrm{~h}$ linear interpoliert. Dieser Prozess ist beispielhaft anhand Abbildung 3.27 a (vor) und $b$ (nach der Schließung) veranschaulicht. Zur Schließung der größeren Lücken bei Tageslicht $\left(R_{G}>10 \mathrm{~W} / \mathrm{m}^{2}\right)$ wurde der Datensatz anschließend nach Temperatur und Bewölkungsgrad klassifiziert und in einem zweiten Schritt mithilfe der Licht-Antwort Funktion nach Michaelis-Menten (Glg. 2.28) lückenbereinigt. Die verwendeten Parameter sind nach Temperaturklassen und Bewölkungsgrad in Tabelle 3.5 zusammengefasst. Zudem ist der gemessene Maximalfluss $\mathrm{F}_{\mathrm{CO}_{2} \max }$ aufgeführt. Die durchweg hohen Werte des Bestimmtheitsmaßes $R^{2}$ deuten darauf hin, dass Temperatur und Bewölkungsgrad als Variable zur Klassifizierung treffend gewählt wurden.

Auf eine jahreszeitliche Trennung des Datensatzes konnte indes verzichtet werden. Alle Untersuchungen haben gezeigt, dass die etwas geringeren Niederschlagsmengen der zweiten Jahreshälfte 2004 in der Regel keinen entscheidenden Einfluss auf die Netto- $\mathrm{CO}_{2}$ Flüsse des Regenwaldbestandes hatten. Abgesehen von den Monaten Sep./Okt. 2004 lassen sich keine bedeutenden saisonalen Schwankungen erkennen. Zur Veranschaulichung zeigt Abbildung 3.28 den - bezügl. der Tageswerte - geschlossenen Datenssatz der NettoKohlendioxidflüsse $\left(F_{C}+F_{S}\right)$. Entlang der Ordinate ist die Tageszeit und 


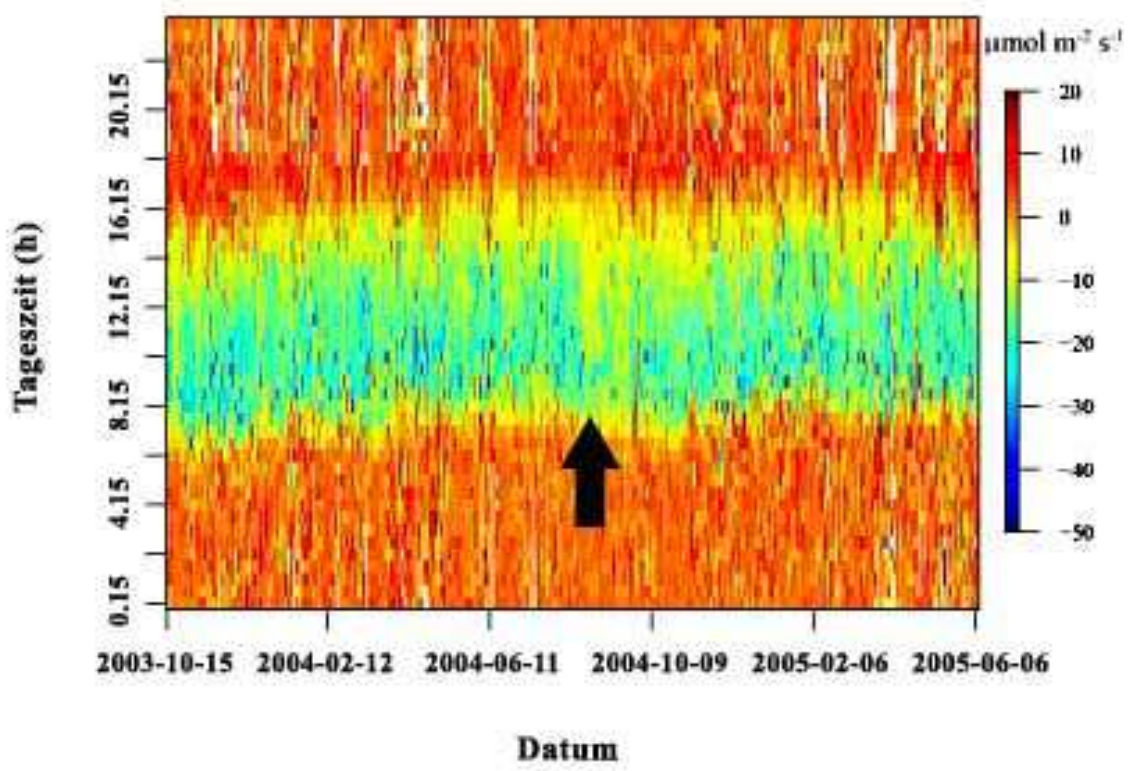

Abbildung 3.28: Saisonale und tageszeitliche Variation des $N E E\left(F_{C}+F_{S}\right)$. Alle Flüsse für $R_{G}>10 \mathrm{~W} / \mathrm{m}^{2}$ sind mit modellierten und interpolierten Werten geschlossen worden. Die nächtlichen Daten sind anschließend nach Anwenden des $u_{*}$-Filterkriteriums durch Zweimonatsmittel ersetzt worden. Der Zeitraum Sep./Okt. 2004 (siehe Pfeil) lässt etwas geringere $\mathrm{CO}_{2}$ Aufnahmeraten erkennen.

entlang der Abzisse das Datum aufgetragen. Die Einheit der dargestellten Netto $\mathrm{CO}_{2}$-Flüsse ist $\mu \mathrm{mol} \mathrm{m} \mathrm{m}^{-2} \mathrm{~s}^{-1}$. Die Behandlung der - noch lückenhaften - Nachtflüsse erfolgte separat und wird im anschließenden Abschnitt näher besprochen. Wie bereits erwähnt, sticht lediglich ein kurzer Zeitraum Sep./Okt. 2004 (gelb gefärbtes Areal - siehe Pfeil) - etwas heraus; hier lassen die assimilatorischen Flüsse etwas geringere Aufnahmeraten erkennen. Es handelt sich erwartungsgemäß genau um den kurzen Zeitraum, der vergleichsweise hohe Wasserdampfdefizit und demzufolge auch hohe Bowen-Ratio Werte aufwies (vergl. Abschn. 3.1.2 und 3.2). In den Vormonaten Jul.-Sep. 2004 gab es nur wenig - zeitweise gar keinen - Niederschlag (vergl. Abb. 3.3 unten). Wahrscheinlich führte die zunehmende Austrocknung des Bodens zu Trockenstresserscheinungen; Stomata-Schließreaktionen des Blattwerks führten in der Folge zu verminderter Fotosyntheseaktivität. Ebenso lagen in diesem Bereich vergleichsweise hohe mittlere Werte der Respiration.

Desweiteren zeigt Abbildung 3.28, dass die $\mathrm{CO}_{2}$-Aufnahme vormittags wesentlich ausgeprägter ist als nachmittags. Die maximale Aufnahme liegt etwa 
bei 10 Uhr lokaler Zeit und stimmt damit sehr genau mit den Messungen von Goulden et al. [GMR ${ }^{+}$04] (im brasilianischen Tiefland, Tapajós, gelegener Regenwald) überein (vergl. auch Abb. 3.17 b). Goulden et al. vermuten, dass der nachmittägliche Abfall auf Schließreaktionen der Stomata, bedingt durch ein hohes Wasserdampfdefizit und höhere Temperaturen am Nachmittag zurückzuführen ist. Beide Argumente stehen im Einklang mit den vorliegenden Ergebnissen hier. Beispielsweise liegt das Wasserdampfdefizit um 14.00h lokaler Zeit um etwa $400 \mathrm{hPa}$ über dem um 10.00h gemessenen Wert (Abb. nicht gezeigt); die Temperatur liegt um $14.00 \mathrm{~h}$ etwa $2.5^{\circ} \mathrm{C}$ höher (vergl. Abb. 3.29). Genauso zeigt sich der Wolkenbedeckungsgrad an Strahlungstagen vormittags stärker ausgeprägt, da dieser naturgemäß stark an das Wasserdampfdefizit gebunden ist.

Insgesamt zeigt sich das Verhältnis von Netto- $\mathrm{CO}_{2}$-Abgabe und Netto- $\mathrm{CO}_{2}$ Aufnahme der Flüsse ziemlich ausgewogen. Nach Abbildung 3.28 findet in $51.3 \%$ aller Fälle Netto- $\mathrm{CO}_{2}$-Abgabe statt (rötlich dargestellt), während in $48.7 \%$ der Fälle $\mathrm{CO}_{2}$ vom Regenwald aufgenommen wird (blau-gelb gefärbt dargestellt). Im Gegensatz zu den Tagflüssen stellen sich die Nachtflüsse als relativ konstante Flüsse heraus (ersichtlich an den gleichmäßig rot gefärbten Arealen). Allein zwischen $18.00 \mathrm{~h}$ und $19.00 \mathrm{~h}$, in der Zeit kurz nach Sonnenuntergang zeigen die Flüsse nach der EK-Methode Maximalwerte ${ }^{12}$ von $5 \mu \mathrm{mol} \mathrm{m} \mathrm{m}^{-2} \mathrm{~s}^{-1}$ (siehe auch Abb. $3.17 \mathrm{~b}$ ).

\subsubsection{Saisonalität der respiratorischen $\mathrm{CO}_{2}$-Flüsse}

Die Bestimmung der respiratorischen Atmungsraten ist von besonderer Bedeutung, da sie zusammen mit den assimilatorischen Flüssen die NettoProduktion des betrachteten Regenwaldbestandes steuern. Mittels der EKMethode können lediglich Veratmungsraten in der Nacht bestimmt werden; insofern sind Tag- und Nachtatmung - wie in Abschnitt 2.2.2 beschrieben getrennt voneinander zu betrachten.

Zur Ermittlung der nächtlichen Respiration wurde zunächst aufgrund der stabilen Schichtungsverhältisse das $u_{*}$-Filter Kriterium angewendet (vergl. Abschnitt 3.3.5). Danach verblieben zur Berechnung nur noch $13.5 \%$ der gemessenen Nachtflussdaten. Die Verteilung der aus der Filterung resultierenden Lücken wies mehr oder weniger starke Unregelmäßigkeiten auf; als Kompromiss zwischen ausreichendem Datenmaterial auf der einen Seite und

\footnotetext{
${ }^{12}$ Man beachte, dass das $u_{*}$-Kriterium zu diesem Zeitpunkt noch nicht angewendet wurde.
} 


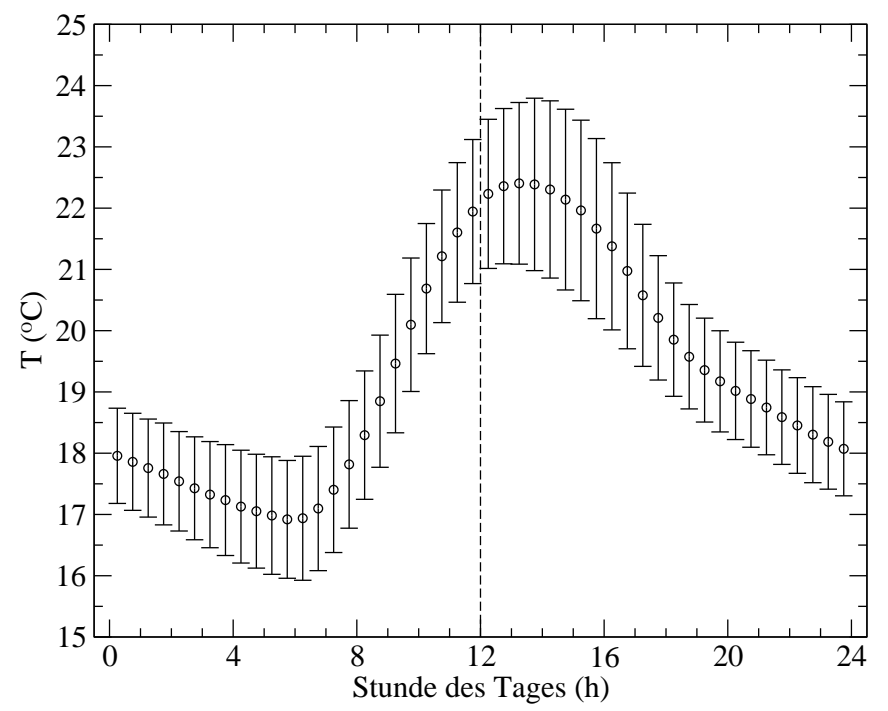

Abbildung 3.29: Mittlerer Tagesgang der Lufttemperatur am Standort Bariri für das Jahr 2004.

hinreichend hoher Auflösung auf der anderen Seite fiel die Wahl auf Mittlungsintervalle von 2 Monaten $\left[\mathrm{IRK}^{+}\right]$. Der saisonale Verlauf der gemittelten Nachtflüsse ist in Abb. 3.30 (schwarz) dargestellt.

Der saisonale Verlauf der Atmungsraten am Tage wurde aus den Erfahrungen, die bei der Modellierung der $\mathrm{CO}_{2}$-Flüsse für Tageswerte $\left(>10 \mathrm{~W} \mathrm{~m}^{-2}\right)$ gewonnen wurden, ermittelt. Den Abbildungen 3.25 (a) und (b) zufolge, weisen die Licht-Antwort Kurven für PPFD-Strahlungswerte $<400$ $500 \mu \mathrm{mol} \mathrm{m} \mathrm{m}^{-2} \mathrm{~s}^{-1} \mathrm{klar}$ lineares Verhaltens auf. Zur Bestimmung des saisonalen Verlaufs von $R_{\text {Tag }}$ scheint es daher angemessen, lineare Regressionen der Form $y_{i}=\alpha x_{i}+\beta+\varepsilon_{i}$ mit $\varepsilon_{i}$ als Störgröße durchzuführen, wobei $\beta$ als $R_{\text {Tag }}$ zu identifizieren ist. Die Intervalllänge betrug entsprechend den Nachtflüssen 2 Monate.

Beim Vergleich der respiratorischen Flüsse zeigt sich, dass die Werte am Tage ausnahmslos höher sind, als in der Nacht. Dies entspricht den Erwartungen, da die Respiration vornehmlich von der Temperatur abhängt.

Trotz einzelner Abweichungen stimmen die respiratorischen Flüsse in ihrem Verlauf gut überein. Von besonderem Interesse (z.B. auch für die Problematik des globalen Klimawandels) sind mögliche Bezüge zu meteorologischen Parametern. Ein Blick auf Abb. 3.3 aus Abschnitt 3.1.2 zeigt einen schwachen 
Bezug zum Niederschlagsverlauf.

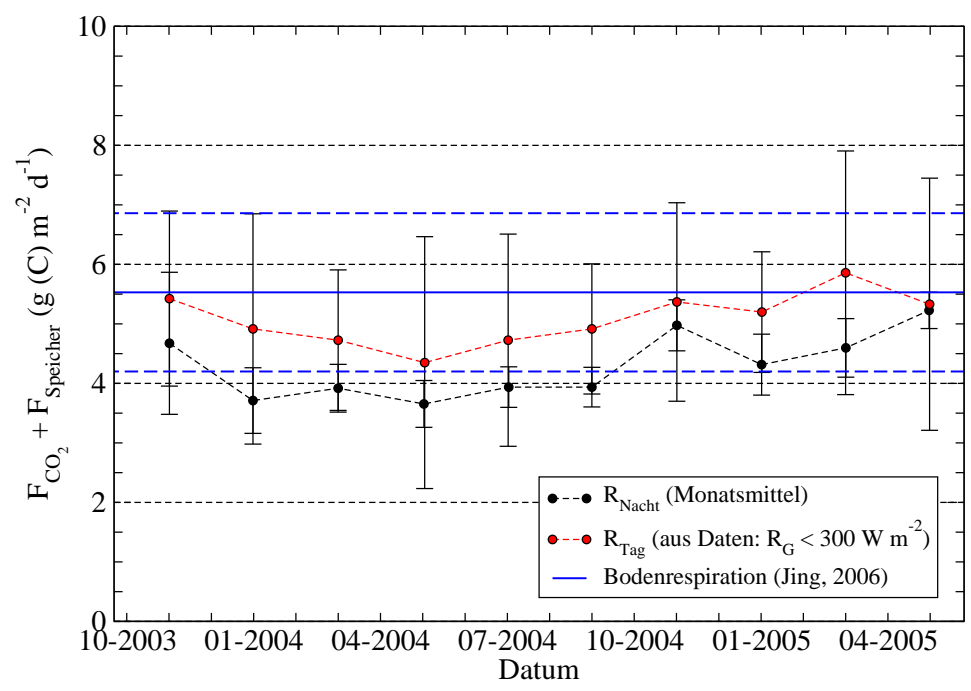

Abbildung 3.30: Saisonaler Gang der Ökosystemrespiration: Die Respiration am Tage $R_{\text {Tag }}$ wurde nach der in [IRK $\left.{ }^{+}\right]$beschriebenen Methode durch linearere Regression aus dem $\mathrm{CO}_{2}$-Fluss in Abhängigkeit von $R_{G}\left(<300 \mathrm{~W} \mathrm{~m}^{-2}\right)$ ermittelt. $R_{N a c h t}$, die sog. dunkle Respiration, geht aus Mittlung der $u_{*}$ gefilterten Nachtwerte hervor. Für beide Methoden betrug die Mittelungslänge zwei Monate. Außerdem ist die Bodenrespiration, welche durch Bodenkammermessungen bestimmt wurde, mit Fehlertoleranz (gestrichene Linie) angegeben [Jin06].

\subsubsection{Berechnung des Nettoökosystemaustausches}

Zur Berechnung des Nettoökösystemaustausches wurde nach den Empfehlungen aus $\left[\mathrm{AGI}^{+} 00, \mathrm{PRA}^{+} 06\right]$ verfahren. Diese sehen im Wesentlichen die Beachtung der durchgeführten Qualitätstests und insbesondere des $u_{*}$ Filterkriteriums vor. Dabei flossen die Ergebnisse der vorliegenden Arbeit in die Berechnung ein, indem systematische und potentielle Fehler, hervorgerufen durch advektive $\mathrm{CO}_{2}$-Flüsse, im Zusammenhang mit dem berechneten $N E E$ kritisch diskutiert wurden.

Zudem wurde ein Vergleich des im Rahmen dieser Arbeit verwendeten 
Planar-Fit Koordinatensystems mit dem vorher in $\left[\mathrm{IRK}^{+}\right]$verwendeten Koordinatensystem nach Mc Millen et al. [McM88] durchgeführt.

\subsubsection{Kumulative Darstellung des Nettoökosystemaustausches und der Komponentenflüsse}

Das $u_{*}$-Kriterium wurde ausführlich in Abschnitt 2.2.2 erläutert. Nach diesem Kriterium wurden die $\mathrm{CO}_{2}$-Flüsse, welche unter unzureichenden Turbulenzbedingungen auftraten, vollständig ersetzt. Indem jedoch lediglich $13.5 \%$ gültige Nachtflüsse zur Darstellung der respiratorischen Flüsse verwendet wurden, ergeben sich im Zuge der Berechnung des $N E E$ im Vergleich zu borealen Waldökosystemen erheblich größere Unsicherheiten $\left[\mathrm{MGM}^{+} 04\right]$. Deswegen scheint es plausibel und notwendig, die Informationen aus den anderen 86.5\% der Nachtdaten nicht vollständig zu vernachlässigen.

Die Abbildungen 3.31 zeigen schließlich zum einen (a) den Verlauf des NEE und zum anderen (b) die einzelnen Komponentenflüsse der Nettoökosystembilanz in kumulativer Darstellung. Bevor näher auf den Vergleich zwischen Planar-Fit und 2d-Rotationsmethode (Abb. 3.31 a, schwarz und rot dargestellt) eingegangen wird, fällt bei Betrachtung der Komponentenflüsse auf, dass sowohl der respiratorische $(R E)$ als auch der assimilatorische $(G P R)$ $\mathrm{CO}_{2}$-Fluss nur leichten saisonalen Schwankungen unterworfen sind. Da es keine ausgeprägten biophysikalischen Steuerungsmechanismen wie ausgeprägte Regen- respektive Trockenzeiten oder auch Temperaturvariationen gab, ist dies auch nicht weiter überraschend.

Interessanterweise wiesen die Bodenhaubenmessungen in Tapajós (Pará, Brasilien) während der Trockenzeit eine um etwa $2 \mu \mathrm{mol} \mathrm{m} \mathrm{m}^{-2} \mathrm{~s}^{-1}$ geringere Bodenrespiration auf $\left[\mathrm{GMR}^{+} 04\right]$; dort korrelierten die respiratorischen Flüsse deutlich positiv mit der Bodenfeuchte. In Bariri trat innerhalb der Messperiode keine ausgeprägte Trockenzeit auf. Für die Regenzeit (1.te Jahreshälfte 2004) bleibt festzustellen, dass unsere Messungen den von Goulden et al. in diesem Punkt nicht folgen. Offensichtlich füllen sich während der Regenzeit die Bodenporen in Bariri derart stark mit Wasser, sodass die Gasdiffusion gehemmt ist. In der Folge könnte es zur Unterversorgung der Mikroorganismen mit Sauerstoff kommen, sodass evtl. weniger $\mathrm{CO}_{2}$ respiriert wird.

\subsubsection{Koordinatensystemvergleich}

Zur Berechnung des NEE wurden zwei verschiedene Koordinatensysteme verglichen: Zum einen das hier standardmäßig verwendete PF-Verfahren und 

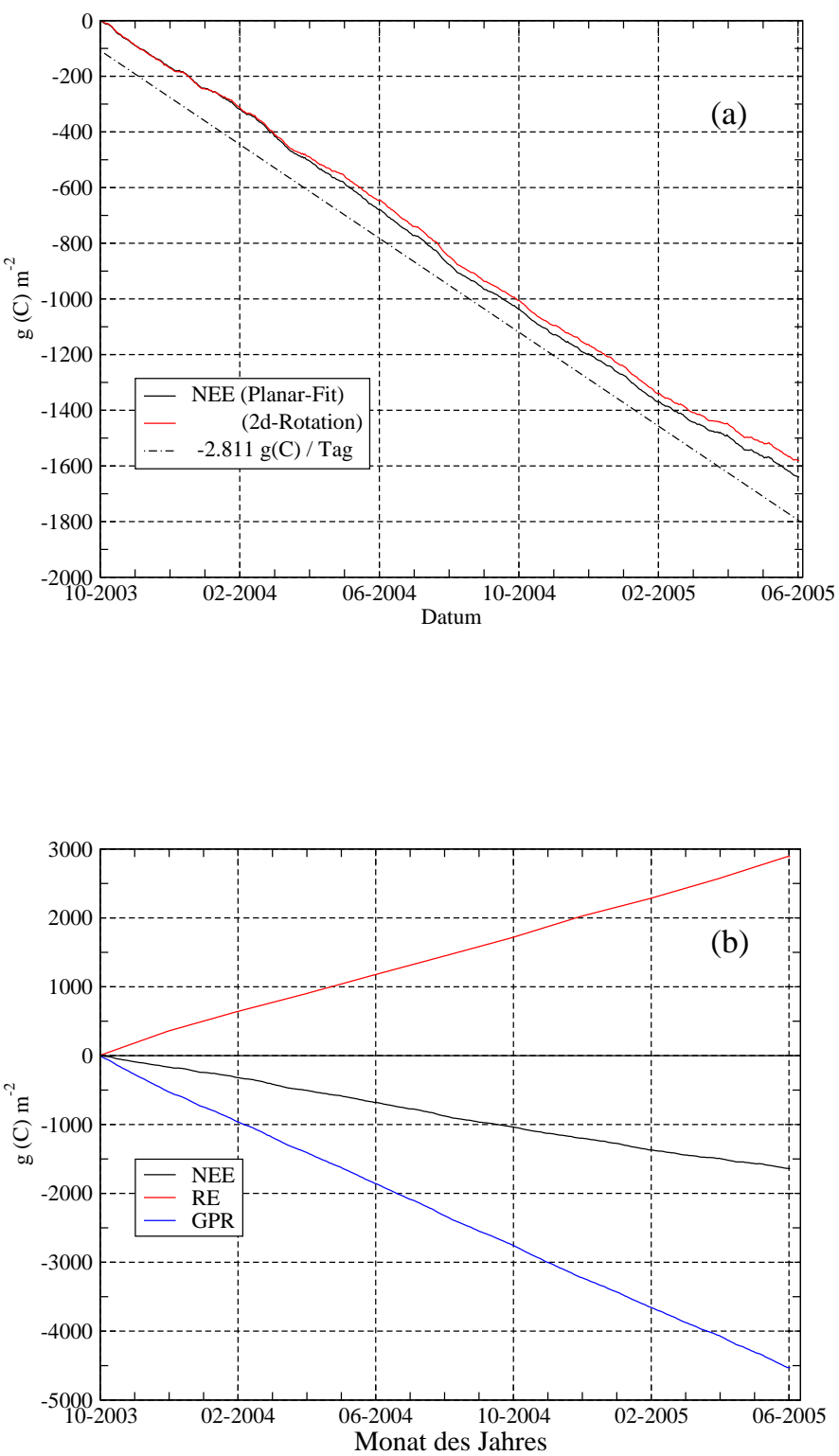

Abbildung 3.31: (a) Kumulativ dargestellter $N E E\left(F_{C}+F_{S}\right)$, berechnet nach dem Planar-Fit und dem 2- $d$ Rotation Verfahren. Die unterbrochene Linie stellt eine Steigung von $-2.81 g(C) / d$ dar. (b) Kumulativ dargestellter $N E E$ (schwarz) als Summe seiner Komponenten $R E$ und GPR. 
zum anderen das $2 d$-Rotationsverfahren. Auf Abbildung 3.32 sind die $\mathrm{CO}_{2}$ Flussbeiträge nach der EK-Methode gegeneinander aufgetragen.

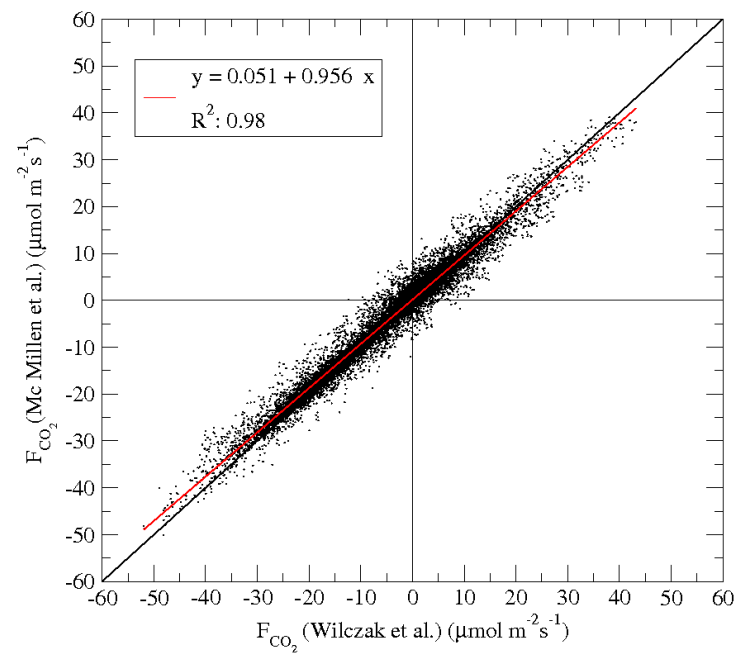

Abbildung 3.32: Vergleich des turbulenten (EK) $\mathrm{CO}_{2}$-Flusses (in $\mu \mathrm{mol} \mathrm{m} \mathrm{m}^{-2} \mathrm{~s}^{-1}$, zum einen berechnet im Planar-Fit Koordinatensystem [WOS01] und zum anderen berechnet im 2- $d$ Rotationssystem [McM88]. Neben der roten Regressionslinie ist schwarz die $1: 1$ Linie dargestellt.

Tabelle 3.6: NEE, berechnet aus den EK-Messungen nach der Planar-Fit als auch nach der 2- $d$ Rotations-Methode. Angegeben ist der kumulierte $\mathrm{CO}_{2^{-}}$ Fluss über den gesamten Zeitraum der Messperiode (15.10.03-07.06.05) als auch die mittlere jährliche Summe in $g(C) m^{-2}$.

\begin{tabular}{lrr}
\hline Methode & jährl. Summe & Summe insges. \\
\hline \hline Planar-Fit & -993.96 & -1639.39 \\
2-d Rotation & -958.31 & -1580.55 \\
\hline
\end{tabular}

Lineare Regression zeigt mit einer Steigung von $0.96,\left(R^{2} \approx 0.98\right)$ eine Tendenz zu höheren Flussbeiträgen auf seiten des PF-Verfahrens. Dies wirkt sich beim Vergleich mit dem 2-d Rotationsverfahren mit einem höheren zeitlich integrierten $\mathrm{CO}_{2}$-Fluss aus: Der jährliche $N E E$ berechnet nach dem 2- $d$ Rotationsverfahren liegt mit $958 g(C) m^{-2} y^{-1}\left[\mathrm{IRK}^{+}\right] 3.6 \%$ unter dem mit der 
PF-Methode $\left(993 g(C) m^{-2} y^{-1}\right)$ berechneten (siehe auch Tab. 3.6) . Tendenziell ähnliche Resultate sind in [LL05] (S. 55ff.) aufgeführt; dort liefert ein zwei Monate langer Datensatz eine 4\% höhere Summe.

\subsubsection{Resultate aus den Berechnungen zur vertikalen Advektion}

Im Folgenden sind nun die vorangegangenen Untersuchungen zur Abschätzung der advektiven $\mathrm{CO}_{2}$-Flüsse miteinzubeziehen. Aus den Berechnungen zur vertikalen Advektion (Abschn. 3.4.1) bei Nacht wurden (unter stabilen atmosphärischen Bedingungen) $204 g(C) m^{-2} y r^{-1}$ ermittelt. Das würde für ein hypothetisches Kontrollvolumen um den Messturm herum bedeuten, dass $204 g(C) m^{-2} y r^{-1}$ vertikal an dem EK-System vorbei aus dem Probevolumen heraus advektiert wird. In der Tat zeigen die Untersuchungen aus Abschnitt 3.4.1, dass sich das typische nächtliche Konvergenzmuster (hangabwärts gerichtete Kaltluftströmung plus nach unten gerichtete Vertikalwindgeschwindigkeit) und damit auch die vertikale Advektion für Werte $u_{*}>0.3$ abzuschwächen beginnt, da die mittlere Vertikalwindgeschwindigkeit immer kleinere Werte annimmt (vergl. Abb. 3.16 b). Insofern scheint sich die Wirksamkeit des Kriteriums zu bestätigen. Desweiteren führt der horizontale Konzentrationsgradient nach den Ausführungen aus Abschn. 3.4.1 vermutlich zu einem weiteren positiven Flussbeitrag, sodass die Abreicherung von $\mathrm{CO}_{2}$ aus dem Kontrollvolumen weiter forciert werden könnte. Die vertikale Advektion wird in diesem Sinne im letzten Abschnitt als "potentieller Flussbeitrag" ausführlich zu diskutieren sein.

Im nächsten Schritt wurden die Ergebnisse aus den $3 d$-Modellierungen berücksichtigt. Die wesentlichen Ergebnisse sind in Abschn. 3.4.2 Abb. 3.24 zusammengefasst. Der normalisierte $\mathrm{CO}_{2}$-Fluss $\mathrm{FCO}_{2}(0,0,48) / \mathrm{FCO}_{2}(-\infty$, $-\infty, 48)$ liefert als reliefbedingte Abweichung vom tatsächlichen $\mathrm{CO}_{2}$-Fluss (in [SL04] auch als "real flux" bezeichnet) am Ort der Messungen eine Möglichkeit zur Bestimmung des systematischen Fehlers des vertikalen Flusses. $\mathrm{Zu}$ berücksichtigen ist, dass die $\mathrm{CO}_{2}$-Flüsse unter der Annahme einer neutral geschichteten Atmosphäre gerechnet wurden.

Zur Berechnung des systematischen Fehlers wurden alle Flussbeiträge (turbulenter plus advektiver Fluss) neutraler und nahe neutraler Schichtung $(-0.1<\varsigma<0.1)$ in einem letzten Post-Processing Schritt windrichtungsabhängig (30-Abschnitte) mit dem Reziproken des normalisierten Flusses multipliziert. In der Summe ergibt sich eine Verringerung der $\mathrm{CO}_{2}$-Aufnahme von $993 g(C) m^{-2} y^{-1}$ um $152 g(C) m^{-2} y^{-1}$.

Unberücksichtigt von der Korrektur blieben damit alle Flüsse aus dem instabilen Schichtungsbereich $(\varsigma \leq-0.1)$. Diese lassen sich weder experimen- 
tell noch mit dem $3 d$-Modell adäquat erfassen. Die Vernachlässigung scheint jedoch vor dem Hintergrund der plausiblen Resultate aus den durchgeführten Qualitätstests sowie der Untersuchungen von [AHY03, FBV03] u.v.a. als gerechtfertigt; letztere Ergebnisse zeigen, dass horizontale und vertikale Advektion bei instabiler Schichtung in der Regel am wenigsten Einfluss auf den Gesamtfluss hat. Zum einen, weil sich horizontale und vertikale Advektion am Tage oftmals kompensieren und zum anderen, weil die advektiven Flüsse aufgrund der starken Durchmischung der Prandtl-Schicht betraglich sehr viel kleiner sind [Sch05]. 


\section{Kapitel 4}

\section{Zusammenfassung und Diskussion}

Die Bestimmung der Kohlenstoffdioxidbilanz stellt insbesondere in tropischen Regionen eine wissenschaftliche Herausforderung dar. Zudem steigen mit der Ausweitung der Messstandorte in immer entlegenere und komplexere Gelände die Anforderungen bei der Durchführung der Fehleranalyse.

Bereits in Ibrom et al. [IRK $\left.{ }^{+}\right]$wurden die $\mathrm{CO}_{2}$-Flussmessungen hinsichtlich des hohen Wertes des Netto-Ökosystemaustauschs (NEE) von $958 g(C) m^{-2}$ $y r^{-1}$ kritisch diskutiert. Das große Problem, speziell bezogen auf tropische Ökosysteme, besteht in der Tatsache, dass ein großer Teil der Nachtdaten aufgrund unzureichender Austauschbedingungen herausgefiltert ( $u_{*}$-Kriterium) werden muss $\left[\mathrm{SMM}^{+} 03\right]$. Mit einer mittleren nächtlichen Respiration von ca. 4-4.5 $\mu \mathrm{mol} \mathrm{m} \mathrm{m}^{-2} \mathrm{~s}^{-1}$ liegt der Standort Bariri, verglichen mit den Messplätzen Reserva Jarú in Róndónia, Brasilien $\left(5-6 \mu \mathrm{mol} \mathrm{m}^{-2} \mathrm{~s}^{-1}\right)\left[\mathrm{GML}^{+} 96\right]$, Cuieiras in Zentral Brasilien $\left(6.6-7.2 \mu \mathrm{mol} \mathrm{m}^{-2} \mathrm{~s}^{-1}\right)$ [FBT $\left.^{+} 02\right]$ oder Tapajós in Pará, Brasilien $\left(8-9 \mu \mathrm{mol} \mathrm{m} \mathrm{m}^{-2} \mathrm{~s}^{-1}\right)\left[\mathrm{MGM}^{+} 04\right]$ am niedrigsten. $\mathrm{Zu}$ berücksichtigen ist, dass es sich bei den aufgezählten Standorten ausnahmslos um Tieflandregenwälder handelt. Ursächlich könnte dementsprechend das im Vergleich zum betrachteten Hochlandregenwald im Schnitt $7^{\circ} \mathrm{C}$ höhere Temperaturniveau sein.

Andererseits ergaben sich, nachdem am Standort Bariri Bodenkammermessungen angeschlossen wurden, signifikante Diskrepanzen zwischen den Werten der nächtlichen Bodenrespiration und den turbulenten Flussmessungen aus $48 m$ Höhe $\left[\mathrm{BHN}^{+}\right.$96, $\mathrm{LRA}^{+} 97, \mathrm{BFW}^{+}$00, MML96]. Dabei wurden aufgrund der relativ großen Unsicherheiten, die mit der Bestimmung der Bodenrespiration (einfaches statisches Kammersystem mit 16 Messkammern [Jin06]) verbunden sind und weil eine große Messkampagne in etwa $50 \mathrm{~km}$ Entfernung auf etwa gleicher Höhe (Wuasa: 1190m üNN) etwa 42\% niedrigere 
mittleren Werte für die Bodenrespiration ergab [Pur05], Fehlerschranken in Höhe $\pm 50 \%$ des eigentlichen mittleren Messwertes von $5.5 \mathrm{~g}(C) \mathrm{m}^{-2} d^{-1} \mathrm{zu}-$ gelassen. Unter Berücksichtigung aller Unsicherheiten konnte dennoch plausibel anhand des starken Unterschiedes der betrachteten Flüsse gezeigt werden, dass sehr wahrscheinlich hangabwärts gerichtete Kaltluftströmungen (drainage flows) für advektiv bedingten Flussverlust verantwortlich sind [LBA99, $\left.\mathrm{ABC}^{+} 05\right]$.

Dies stützen ebenso die Untersuchungen zur vertikalen Advektion [Lee98]. Mit $204 g(C) m^{-2} y^{-1}$ scheint ein beträchtlicher Teil des Kohlenstoffflusses in der Nacht vertikal am Sensor vorbeiadvektiert zu werden. Mithilfe der Untersuchungen zum lokalen Windsystem (Abschn. 3.1.1) ließen sich die nächtlichen Strömungsverhältnisse gut analysieren. Die gravitativ angetriebene Kaltluftströmung fließst vorwiegend aus südwestlicher Richtung den Hang hinab; turbulente Luftbewegungen sind stark unterdrückt und die Vertikalwindkomponente weist stets in den Bestand hinein ${ }^{1}$. Der Eintrag von $\mathrm{CO}_{2}$-ärmerer $\mathrm{Luft}^{2}$ aus der Schicht über dem Bestand führt also zu einer Abreicherung höherkonzentrierter Bestandesluft. Bezüglich der horizontalen Advektion wiesen Modellergebnisse aus $\left[\mathrm{ABC}^{+} 05\right]$ in Verbindung mit den Boden-Quellgebietsuntersuchungen am Standort [Jin06] auf einen weiteren positiven $^{3}$ Flussbeitrag hin.

Insbesondere die 2005 in Renon (Norditalien, 1760m üNN, Hangneigung $\leq 10 \%$ ) durchgeführten Untersuchungen (3d-Setup) scheinen die vorangegangenen Aussagen zusätzlich zu stützen $\left[\mathrm{MCM}^{+} 05\right]$. Ebenso wie am Standort Bariri war die Strömungsrichtung infolge der nächtlichen Kaltluftströmung innerhalb des Bestandes ausnahmslos hangabwärts gerichtet. Und ebenso ergaben die Messungen einen sehr kleinen normalisierten Speicherfluss sowie positive vertikale und horizontale Advektion. Unter diesen Voraussetzungen lässt sich die durchgeführte Reduzierung des nächtlichen Strömungsregimes auf zwei Dimensionen am Standort Bariri sehr gut rechtfertigen. Zwar werden aufgrund der Simplifizierung möglicherweise nicht alle Strömungsereignisse detektiert. Diverse Messkampagnen an verschiedenen CARBOEUROFLUX Standorten ${ }^{4}$ (Renon 2002/03, Italien; Bily Criz 2003,

\footnotetext{
${ }^{1}$ Gilt vor allem für Vertikalwinde der stationären Nachtwindrichtungen $\approx 240^{\circ}$; bei Winden aus Richtungen zwischen $250^{\circ}$ und $280^{\circ}$ handelt es sich um Übergangszeiten (neutrale Schichtung), in deren Verlauf sich die Windrichtung ändert.

${ }^{2} \mathrm{Bei}$ stabil geschichteter Atmosphäre in der Nacht ist der vertikale $\mathrm{CO}_{2}$ Konzentrationgradient stets negativ.

${ }^{3}$ Entsprechend der Konvention, dass $\mathrm{CO}_{2}$-Flüsse aus dem Bestand heraus positiv gezählt werden, bedeutet "positiv" - auf das Kontrollvolumen bezogen -, dass $\mathrm{CO}_{2}$ abgereichert wird.

${ }^{4} \mathrm{http}: / /$ www.bgc-jena.mpg.de/public/carboeur/
} 
Tschechien; Hesse 2003, Frankreich; Weidenbrunnen 2003, Deutschland) haben jedoch gezeigt, das die wesentlichen - für den $\mathrm{CO}_{2}$-Fluss - relevanten Prozesse erfasst werden [AHY03].

Dabei ist zu betonen, dass es sich um einen potentiellen Flussbeitrag handelt. In den vorangegangenen Abschnitten ließ sich zeigen, dass die Vertikalwindgeschwindigkeit als dominierender Faktor für Vertikaladvektion mit zunehmender Turbulenz abnimmt. Durch das $u_{*}$-Filterkriterium ist somit gewährleistet, dass der durch unberücksichtigte vertikale Advektion verursachte Fehler, minimiert wird. Dies gilt sehr wahrscheinlich auch für horizontale Advektion, die im Rahmen dieser Untersuchungen nicht berücksichtigt werden konnte. Einzelne Experimente, in denen vertikale als auch horizontale Advektion gemessen wurde, lassen sich an dieser Stelle beispielhaft anführen $\left[\mathrm{ABC}^{+} 05, \mathrm{LH} 02, \mathrm{ML02}, \mathrm{AHY03}\right.$.

Hinzuzufügen ist, dass der modellierte Speicherterm für die vertikale Advektion einen Maximalwert festlegt. Denn indem das Modell von Oltchev $\left[\mathrm{OCN}^{+} 02\right]$ advektive Einflüsse in der Nacht tendenziell eher unterschätzt, wie sich bei Vergleichen mit ersten Profilmessungen herausstellte, werden Konzentrationsdifferenzen der Form $\langle C\rangle-C$ (vergl. Glg. 2.8) vermutlich eher überschätzt.

Ob der Standort Bariri auch am Tage von advektiven Prozessen betroffen ist, lässt sich mithilfe der konventionellen Methoden schwer beurteilen. Hinweise lieferten die obligatorischen Qualitätstests aus [Fok03, FW96, VM97]. So ließen nach dem Modell der integralen Turbulenzcharakteristik insbesondere die Tagesflüsse einen ausreichend hohen Turbulenzgrad und hinreichend homogene Unterlageneigenschaften zur Durchführung von turbulenten Flussmessungen erkennen. Da das Turbulenzmodell für ebenes homogenes Gelände entwickelt wurde, könnte die gute Übereinstimmung der Messungen aus zwei verschiedenen Messhöhen außerdem daraufhinweisen, dass sich die orografischen Geländeverhältnisse weniger stark auf die Flussmessungen auswirken. Auch zeigt die Untersuchung von Varianzspektren [IRK ${ }^{+}$], dass im Wesentlichen alle relevanten Skalen erfasst wurden. Die gut geschlossene Energiebilanz weist allgemein auf die hohe Qualität der durchgeführten Messungen hin. Jedoch sollte an dieser Stelle nicht direkt auf die Qualität der $\mathrm{CO}_{2}$-Flüsse geschlossen werden. Denn speziell in der Nacht hängt die Größenordnung der advektiven Flussverluste sehr spezifisch vom betrachteten Skalar respektive von der physikalischen Zustandsgröße ab. Bedingt durch die starken respiratorischen Quellen kommt es auf seiten des Kohlendioxids zu ausgeprägten vertikalen Gradienten, während der Temperaturgradient in der Nacht vergleichsweise kleine Werte annimmt.

Die Quellgebietsanalyse stellt eine wichtige Voraussetzung für EK-Messungen dar. Aufgrund der schwierigen Geländebedingungen wurde sie deshalb mit 
großem Aufwand betrieben. Zwei verschiedene Modelle wurden verwendet. Zur Bestimmung der reliefbedingten Beeinflussung des Quellgebiets das Modell von Sogachev [SL04] (E- $\omega$ Modell) und zur Bestimmung der Quellgebietsausdehnung unter stabiler atmosphärischer Schichtung sowie zum Vergleich das analytische Modell von Kormann und Meixner [KM01]. Die Analysen ergaben, dass sich der Quellgebietsbereich unter dem Einfluss des unebenen Geländes entsprechend des veränderten Anströmfeldes zwar verlagert, aber nicht signifikant ausdehnt oder verkürzt. Unter diesen Voraussetzungen lag es nahe, die Untersuchungen für die größte Quellgebietsausdehnung mit dem analytischen Modell von Kormann und Meixner unter stabilen atmosphärischen Schichtungsbedingungen anzuschließen. Lediglich Flüsse aus östlichen Richtungen schienen die Bedingung eines homogenen Anströmfeldes (fetch) zu verletzen. Hier wechselte die Vegetation von Wald zu Kräuterwiese. Da jedoch Nachtflüsse aus östlichen Richtungen am Standort nicht vorkommen (nahezu alle Nachtflüsse stammen aus südwestlicher Richtung), bleibt festzustellen, das die turbulenten Flüsse nicht von Fetch-Verletzungen betroffen zu sein scheinen.

Ein weiteres Ergebnis aus den Quellgebietsuntersuchungen bestand in dem Nachweis von Flussbeitrag aus dem windabgewandten Bereich des Turmstandorts $^{5}$. Dabei stellte sich heraus, dass besonders Winde aus östlichen Richtungen eine leichte Ausdehnung des Quellgebiets in den Lee-Bereich des Turmes bedeuten. Deswegen überrascht auch nicht, dass gerade in diesem Windsektorbereich die Planar-Fit Ebene bei höheren Windgeschwindigkeiten systematische Abweichungen vom orografischen Verlauf des Geländes zeigt. Für die Wahl zukünftiger Messstandorte lässt sich daher folgern, dass Messungen möglichst weit entfernt des höchsten Punktes einer Reliefwelle oder eines Hügels stattfinden sollten. Unproblematischer scheint moderat geneigte Topografie, welche in möglichst weiten Teilen monoton verlaufen sollte.

Insgesamt scheint das Modell nach Sogachev et al. [SMHL02] durchweg plausible Ergebnisse zu liefern. Dennoch bleibt die Frage nach der Anwendbarkeit respektive den Einschränkungen von Modellen, die auf der $K$-Theorie basieren und zur Beschreibung von atmosphärischer Turbulenz innerhalb und über hoher Vegetation verwendet werden. Einige Besonderheiten von atmosphärischen Strömungen über hoher Vegetation sind in diesem Kontext kontrovers zu diskutieren: Inhomogenitäten der vertikalen Geschwindigkeitsstatistik, Transportprozesse durch sogenannte Sweeps/Ejections (kohärente Transportbewegungen) und räumlich verteilte Quellen/Senken von Impuls und Skalaren [WFR98]. Demzufolge stellen einige Autoren die Anwendbar-

\footnotetext{
${ }^{5}$ Vergleichbare Ergebnisse ließen sich beispielsweise den Modellierungen eines finnischen Untersuchungsgebietes nahe Hyytiälä entnehmen [SRV04].
} 
keit der $K$-Theorie innerhalb der Vegetation in Frage [DB94, Fin00, KA98]. Nichtsdestoweniger, seit den ersten durchgeführten Modellrechnungen versuchen Sogachev et al. mit Nachdruck das Modell zu validieren. Zahlreiche Vergleiche mit den gängigen analytischen und LS Footprint Modellen auf der einen Seite und EK-Messungen auf der anderen Seite scheinen den Einsatz des Modells gut zu rechtfertigen [SL04, SMHL02, SRV04, SPGV05].

Aber es existieren noch weitere Gründe, die für einen anderthalbfachen Schließungsansatz sprechen. Denn beispielsweise auch mithilfe der LES (Large Eddy Simulation), welche für nichtlokale Transportprozesse wesentlich besser geeignet scheint, lassen sich nicht zwangsläufig realitätsgetreuere Ergebnisse erzielen. Die Schwierigkeit im Zusammenhang mit dieser Methode besteht in dem Auffinden einer geeigneten Parametrisierung für die Vielzahl an Konstanten und der rasch zunehmenden Turbulenz sehr nahe am Boden [WY03]. Außerdem sind abgesehen von den unpraktikabel langen Rechenzeiten und mit der Suche einer geeigneten Mittelungszeit zusätzlich Unsicherheiten verbunden [Dea78]. So erwies sich im Rahmen eines Modellvergleichs [YM06, SP06] das Modell von Sogachev et al. unter Berücksichtigung desselben experimentellen Datensatzes als das exaktere; die mittlere Strömungscharakteristik über einem modellierten Wiese-Wald Übergang konnte aufgrund der höheren Auflösung insgesamt besser wiedergegeben werden.

Der Anwendungsbereich $K$-Theorie basierter Modelle hängt nach Finnigan und Belcher [FB04] wesentlich von der Vegetationstruktur der betrachteten Wälder ab. Dabei ist von großer Bedeutung, dass die räumliche Variation von $c_{d} A$ (Gleichg. 2.39), verglichen mit der Skale der großen energiereichen Wirbel, die für die Transportprozesse verantwortlich sind, langsam verläuft. Beispiele für Experimente, bei denen der Skalartransport mit dem Modell von Sogachev et al. innerhalb der Vegetation treffend beschrieben wurde, sind [SMHL02] und [KS04].

Zum Schluss werden der Unsicherheiten verbunden mit den EK-Messungen im Sinne von Moncrieff et al. [MML96] als vollständig und selektiv systematische Fehler interpretiert. Der Ausdruck "selektiv" in diesem Sinne bezeichnet einen Fehler, der lediglich zu bestimmten Tages- oder Nachtzeiten auftritt. Die advektiven Nachtflüsse treten offensichtlich selektiv systematisch auf. Bei Tage wurde festgestellt, dass die gemessenen $\mathrm{CO}_{2}$-Flüsse in Abhängigkeit von der Windrichtung über- oder unterschätzt werden. Zwar handelt es sich auch um selektiv systematische Fehler. Für den Fall neutraler Schichtungsverhältnisse kompensieren sie sich jedoch zum Teil.

Der selektiv systematische Fehler in der Nacht wurde im vorherigen Abschnitt als potentieller Fehler bezeichnet. Er tritt nur dann voll in Erscheinung, wenn das $u_{*}$-Filterkriterium nicht angewendet wird. Tatsächlich ergaben die Untersuchungen, dass sich die mittlere Senkenstärke des Waldökosystems um 
$192 g(C) m^{-2} y^{-1}$ erhöht, wenn das Filterkriterium nicht auf die turbulenten Flussmessungen angewendet wird. In diesem Sinne stützen die durchgeführten Untersuchungen zur vertikalen Advektion bei Nacht die Plausibilität des $u_{*}$-Filterkriteriums.

Entsprechend der Empfehlung in [MML96] wird der berechneten Nettoökosystemaustauch vollständig mit Fehler in der Form 993-152 $\pm 46 g(C) \mathrm{m}^{-2} \mathrm{yr}^{-1}$ angegeben, wobei der 2.te Term die systematische und der 3.te die zufällige Abweichung angibt. Die Tendenz den Kohlendioxidfluss systematisch zu überschätzen wird mit dem Minuszeichen angezeigt.

In keinem der bisher existierenden Langzeituntersuchungen verschiedener Waldökosysteme wurden derart hohe jährliche Summen von beinahe $1000 \mathrm{~g}$ (C) $\mathrm{m}^{-2}$ beobachtet. Einen Überblick liefert Falge et al. $\left[\mathrm{FBT}^{+} 02\right]$. Dabei reichen die bisher durchgeführten $\mathrm{CO}_{2}$-Flussmessungen von 212 bis $-757 \mathrm{~g}(C) \mathrm{m}^{-2} \mathrm{yr}^{-1}$. Der einzig betrachtete tropische Regenwald in [FBT $\left.{ }^{+} 02\right]$ stellt eine Kohlenstoffsenke von $608 g(C) m^{-2} y^{-1}$ dar [MBJ99]. Die meisten der anderen bereits diskutierten tropischen Langzeituntersuchungen stellen moderate Senken, z.B. $-100 g(C) m^{-2} y^{-1}$ (Reserva Jarú, Róndónia, Brasilien) $\left[\mathrm{GML}^{+}\right.$96] respektive noch schwächere Quellen, z.B. $40 g(C) m^{-2} y^{-1}$ (Tapajós , Pará, Brasilien) $\left[\mathrm{MGM}^{+} 04\right]$ dar. Als besonders herausstechend nimmt sich die Studie von Löscher et al. [LC03] aus. In drei aufeinanderfolgenden Jahren entwickelte sich der Regenwald in La Selva, Costa Rica (1988) von einer leichten Quelle ( 5 bis $133 g(C) m^{-2} y r$ ), über eine moderate Senke im Jahre $1999\left(-153\right.$ bis $\left.-314 g(C) m^{-2} y r\right)$ hin zu einer starken Senke $(-597$ bis $\left.-792 g(C) m^{-2} y r\right)$ im Jahr 2000. Die Gründe für diese Differenzen sind nicht eindeutig. Löscher et al. führen die Variabilität auf El Niño Effekte zurück.

Insofern bleibt fraglich, ob sich die hohen $\mathrm{CO}_{2}$-Aufnahmeraten auf den betrachteten Zeitraum beschränken - wofür es bisher keinen triftigen Anhaltspunkt gibt-, oder ob auch in der Zukunft mit ähnlich hohen Aufnahmeraten zu rechnen ist. Zur Aufklärung werden die durch diese Arbeit angestoßenen Advektionsmessungen am Standort Bariri und Messungen von Biomasseänderungen beitragen. Eine mögliche ökophysiologische Interpretation der Ergebnisse liefern wir in $\left[\mathrm{IRK}^{+}\right]$. 


\section{Anhang A}

\section{Notation}

\section{A.1 Symbolverzeichnis für das E- $\omega$-Modell}

$\begin{array}{cl}A & \text { Blattflächendichte }(L A D) \\ C & C O_{2} \text {-Hintergrundkonzentration } \\ C_{i n} & C O_{2} \text {-Konzentration der interzellularen Zwischenräume } \\ C_{1}, C_{2} & \text { Konstanten für das Turbulenzmodell } \\ C_{\mu} & \text { Konstante für das Tubulenzmodell }(\approx 0.08) \\ C_{2}^{*} & \text { modifizierter Dämpfungskoeffizient } C_{2} \\ c_{d} & \text { Reibungskoeffizient an Blattoberflächen } \\ D & \text { durchschnittlicher Blattdurchmesser } \\ E & \text { turbulente kinetische Energie } \\ f_{I} & \text { Coriolisparameter } \\ K_{\phi} & \text { Austauschkoeffizient der Eigenschaft } \phi \\ L_{0} & \text { Blattflächenindex } \\ l & \text { turbulente Mischungsweglänge } \\ r_{a} & \text { aerodynamischer Transferwiderstand an der Blattoberfläche } \\ r_{s} & \text { stomatarer Transferwiderstand an der Blattoberfläche } \\ S & \text { Verformungs- und viskose Reibungskräfte } \\ S_{C} & \text { Quellterm für } C O_{2} \\ S_{d} & \text { Dissipation durch Reibungseffekte am Blattwerk } \\ U_{g} & \text { geostrophische Windgeschwindigkeit } \\ \alpha & \text { totale / projizierte Blattoberfläche } \\ \beta_{d} & \text { Energieverluste durch Interaktion mit der Waldvegetation } \\ \epsilon & \text { Dissipation der turbulenten kinetischen Energie } \\ \sigma_{E} & \text { Prandtlzahl für turbulente kinetische Energie } \\ \sigma_{\omega}, \sigma_{C} & \text { Schmidtzahl für } \omega \text { und } C \\ \omega & \text { spezifische Dissipation der turbulenten kinetischen Energie }\end{array}$




\section{A.2 Allgemeines Symbolverzeichnis}

A transmittierte Strahlungsleistung

BHD Brusthöhendurchmesser (Stammdurchmesser in 1.3 m Höhe über dem Boden)

$\mathrm{C} \quad \mathrm{CO}_{2}$-Konzentration

$c_{p} \quad$ spezifische Wärme bei konstantem Druck

$c \quad$ Schallgeschwindigkeit

d Versatzhöhe (Parameter des Windprofils)

dir Windrichtung

E turbulente kinetische Energie

Eu Euler-Zahl

$\mathrm{F}_{\mathrm{C}} \quad$ turbulenter Fluss von $\mathrm{CO}_{2}$

$F_{S} \quad$ Speicherfluss von $\mathrm{CO}_{2}$

$F_{a d v} \quad$ advektiver Fluss von $\mathrm{CO}_{2}$

$F_{\text {Sat }} \quad$ Brutto-Fotosyntheserate bei Lichtsättigung

$f \quad$ Frequenz

$G \quad$ geostrophische Windgeschwindigkeit

$G_{t} \quad$ Änderungsrate der im Wald u.d. Bestandesluft gespeicherten Energie

GPR Brutto-Fotosyntheserate (gross photosynthetic rate)

$g \quad$ Schwerebeschleunigung

$H \quad$ Flussdichte fühlbarer Wärme

$K \quad$ turbulenter Austauschkoeffizient (eddy diffusivity)

$L A I \quad$ Blattflächendichte

$L \quad$ Monin-Obukhov Länge

$m_{a} \quad$ Molekulargewicht trockener Luft

$m_{v} \quad$ Molekulargewicht feuchter Luft

$N_{d} \quad$ Niederschlagsmenge

NEE Nettoökosystemaustausch (netto ecosystem exchange)

$P_{e} \quad$ Partialdruck

PPFD fotosynthetisch wirksame Strahlung (photosynthetic photon flux density)

$p \quad$ Luftdruck

$Q_{10} \quad$ Parameter der Temperaturabhängigkeit von $R_{E}$

$q \quad$ spezifische Feuchte

$R \quad$ universelle Gaskonstante

$R_{d f} \quad$ diffuse Strahlung

$R_{\text {Tag }} \quad$ Ökosystematmung bei Tage 


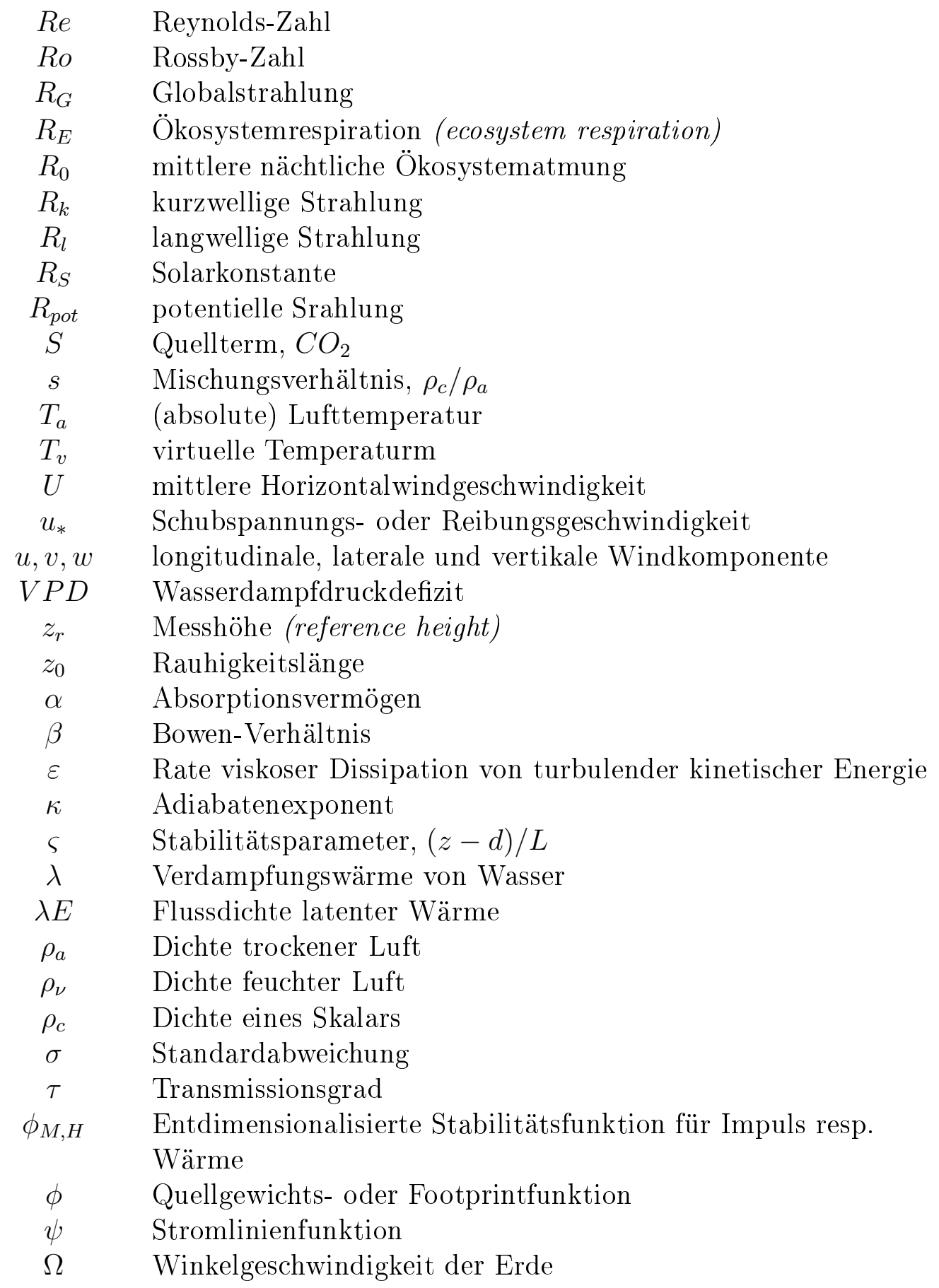




\section{Literaturverzeichnis}

[ABC +05$]$ Aubinet, M. ; Bernhofer, C. ; Cescatti, A. ; Gruenwald, T. ; Havrankova, K. ; Heinesch, B. ; Longdoz, B. ; Marcolla, B. ; Montagnani, L.: Comparing night $\mathrm{CO} 2$-storage and advection at different CARBOEUROFLUX sites. In: Bound. Layer Meteorol. 116 (2005), S. 63-94

[ACE $\left.{ }^{+} 03\right]$ Aubinet, M. ; Clement, R. ; Elbers, J. E. ; Foken, T. ; Grelle, A. ; Ibrom, A. ; Moncrieff, J. ; Pilegaard, K. ; Rannik, Ü ; Rebmann, C.: Methodology for data acquisition, storage, and treatment. Springer Verlag, Heidelberg, 2003.9-35 S.

[AGI $\left.{ }^{+} 00\right]$ Aubinet, M. ; Grelle, A. ; Ibrom, A. ; Rannik, Ü ; Moncrieff, J. ; Foken, T. ; Kowalski, A. ; Martin, P. ; Berbigier, P. ; Bernhofer, C. ; Clement, R. ; Elbers ; J., A. Granier ; Grunwald, T. ; Morgenstern, K. ; K.Pilegaard ; Rebmann, C. ; Snijders, W. ; Valentini, R. ; Vesala, T.: Estimates of the annual net carbon and water exchange of european forests: The EUROFLUX Methodology. In: Adv. Ecol. Res. 30 (2000), S. 113-175

[AHY03] Aubinet, M. ; Heinesch, B. ; Yernaux, M.: Horizontal and vertical CO2 advection in a sloping forest. In: Boundary-Layer Meteorol. 108 (2003), S. 397-417

[AMGD00] Kapitel Relationships between photosynthesis, carbohydrate metabolism and respiration. In: AtKin, O.K. ; MillaR, A.H. ; Gardeström, P. ; Day, D.A.: Photosynthesis: Physiology and Metabolism (Advances in Photosynthesis). 9th. Kluwer Academic Puplishers, Dordrecht, 2000, S. 153-175 
$\left[\mathrm{BCB}^{+}\right]$Black, T.A. ; Chen, W.J. ; Barr, A.G. ; Arain, M.A. ; Chen, Z. ; Nesic, Z. ; Hogg, E.H. ; Neumann, H.H. ; Yang, P.C.: Increased carbon sequestration by a boreal deciduous forest in years with a warm spring. In: Geophys. Res. Lett. 27

[BEF ${ }^{+}$06] Baldocchi, D. ; Elbers, E. ; Falge, E. ; Feigenwinter, C. ; Granier, A ; Heinesch, B. ; Kolle, O. ; Longdoz, B. ; PAPAle, D.: Eddy covariance flux measurements, International Meeting, Gembloux (Belgium). july 2006

[Ber95] BernhaRDT, K.: Zur Interpretation der Monin Obukhovschen Laenge. In: Met. Z. 4 (1995), S. 81-82

$\left[\mathrm{BFW}^{+} 00\right]$ Baldocchi, D. ; Finnigan, J. ; Wilson, K. ; PaW, K.T. ; FALGE, E.: On measuring net ecosystem carbon exchange over tall vegetation on complex terrain. In: Bound. Layer Meteorol. 96 (2000), S. 257-291

[BHN ${ }^{+}$96] Black, T. A. ; Hartog, G. den ; Neumann, H. H. ; Blanken, P. D. ; Yang, P. C. ; Russell, C. ; Nesic, Z. ; Lee, X. ; Chen, S. ; Staebler, R. ; Novak, M. D.: Annual Cycles of Water Vapour and Carbon Dioxide Fluxes in and above a Boreal Aspen Forest. In: Global Change Biol. 2 (1996), S. $219-229$

[Ble01] BlEyL, M.: Experimentelle Bestimmung der Depositionsgeschwindigkeit luftgetragener Partikel mit Hilfe der Eddy Kovarianz Methode ü̈er einem Fichtenbestand im Solling. Georg-August-Universität-Göttingen, Bioklimatologie, Diss., 2001. - 106 S.

[BTSP79] Broeker, W.S. ; Takahashi, T. ; Simpson, H.J. ; Peng, T.H.: Fate of fossil fuel carbon dioxide and the global carbon budget. In: Science 206 (1979), S. 409-410

[CN98] Campbell, G. S. ; Norman, J.: An Introduction to Environmental Biophysics, 2nd. Ed. Springer Verlag, New York, 1998

[DB94] Denmead, O. T. ; Bradley, E. F.: Flux Gradient Relationship in a Forest Canopy. In: The Forest Atmosphere Interaction (1994), S. 421-442 
[Dea78] DEARDORFF, J.W.: Efficient prediction of ground surface temperature and moisture with inclusion of a layer of vegetation. In: J. Geophys. Res. 83 (1978), S. 1889 -1903

[Die07] DiETz, J.: Rainfall partitioning in differently used montane rainforests of Central Sulawesi, Indonesia. Georg-August-Universität-Göttingen, Diss., 2007. - 106 S.

[Fal97] FALK, M.: Turbulente Flüsse von CO2, H2O und fühlbarer Wärme über und in einem Fichtenwald im Solling. Georg-August-Universität-Göttingen, 1997

[Fal04] FALK, U.: Turbulent Fluxes of CO2, H2O an Energy in the Atmospheric Boundary Layer above Tropical Vegetation investigated by Eddy-Covariance Measurements.

Bioclimatology, Georg-August-Universität-Göttingen, Diss., 2004. - 99 S.

[FB95] Finnigan, J.J. ; BRUnet, Y.: Turbulent airflow in forests on flat and hilly terrain. In: Wind and Trees. Cambridge University Press, 1995. - 3-41 S.

[FB04] Finnigan, J. J. ; BelChER: Flow over a hill covered with a plant canopy. In: Quart. J. Roy. Meteorol. 130 (2004), S. 1-29

[FBO $\left.{ }^{+} 01\right]$ Falge, E. ; Baldocchi, D. ; Olson, R. ; Anthoni, P. ; Aubinet, M. ; Bernhofer, C. ; Burba, G. ; Ceulemans, R. ; Clement, R. ; Dolman, H. ; Granier, A. ; Gross, P. ; Grunwald, T. ; Hollinger, D. ; Jensen, N. O. ; Katul, G. ; Keronen, P. ; Kowalski, A. ; Lai, C. T. ; LaW, B. E. ; Meyers, T. ; MoncriefF, H. ; Moors, E. ; Munger, J. W. ; Pilegaard, K. ; Rannik, Ü ; Rebmann, C. ; Suyker, A. ; Tenhunen, J. ; Tu, K. ; Verma, S. ; Vesala, K. T. a.

T. andWilson ; Wofsy, S.: Gap filling strategies for defensible annual sums of net ecosystem exchange. In: Agric. For.

Meteorol. 107 (2001), S. 43-69

[FBT ${ }^{+}$02] Falge, E. ; Baldocchi, D. ; Tenhunen, J. ; Aubinet, M. ; Bakwin, P. ; Berbigier, P. ; Bernhofer, Ch. ;

Bonnefond, J.-M. ; Burba, G. ; Clement, R. ; Davis, K.J. ; Elbers, J.A. ; Falk, M. ; Goldstein, A.H. ; Grelle, A. ; Granier, A. ; Grünwald, T. ; GÖmundsson, J. ; Hollinger, D. ; Janssens, I. ; Keronen, P. ; Kowalski, A. ; Katul, G. ; Law, B. ; Malhi, Y. ; Meyers, T. ; Monson, 
R. ; Moors, E. ; Munger, J.W. ; Oechel, W. ; Paw, U K. ; Pilegaard, K. ; Rannik, U. ; Rebmann, R. ; Suyker, A. ; Thorgeirsson, H. ; Tirone, G. ; Turnipseed, A. ; Wilson, K. ; Wofsy, S.: Seasonality of ecosystem respiration and gross primary production as derived from FLUXNET measurements. In: Agricultural and Forest Meteorology (2002)

[FBV03] Feigenwinter, C. ; Bernhofer, C. ; Vogt, R.: The influence of advection on the short term CO2-budget in and above a forest canopy. In: Boundary-Layer Meteorol. 113 (2003), S. 201-224

[Fin99] Finnigan, J.: A comment on the Paper by Lee (1998): On Micrometeorological Observation of Surface-Air Exchange over Tall Vegetation. In: Agric. For. Meteorol. 97 (1999), S. 55-64

[Fin00] Finnigan, J. J.: Turbulence in Plant Canopies. In: Annu. Rev. Fluid Mech. 32 (2000), S. 519-571

[Fin04] Finnigan, J.J.: A re-evaluation of long-term flux measurement techniques. Part 2. Coordinate systems. In: Boundary-Layer Meteorol. 113 (2004), S. 1-42

[Fok03] Foken, T.: Angewandte Meteorologie, Mikrometeorologische Methoden. Springer, Heidelberg, 2003. - 289 S.

[FW96] Foken, T. ; Wichura, B: Tools for Quality Assessment of Surface-Based Flux Measurements. In: Agric. For. Meteorol. 78 (1996), S. 83-105

[Gar99] Garratt, J.R.: The Atmospheric Boundary Layer. Cambridge University Press., 1999. - 311 S.

[Gas86] GAsH, JHC: A note on estimating the effect of a limited fetch on micrometeorological evaporation measurements. In:

Boundary-Layer Meteorol. 35 (1986), S. 409-414

[GFB $\left.{ }^{+}\right]$Gu, L. ; Falge, E. ; Boden, T. ; Baldocchi, D.D. ; Black, T.A. ; Saleska, S.R. ; Suni, T. ; Verma, S.B. ; Vesala, T. ; WofsY, S.C. ; XU, L.: Objective threshold determination for nighttime eddy flux filtering. In: Agric. Forest Meteorol 128

[GMF+96] Goulden, M.L. ; Munger, J.W. ; Fan, S.-M. ; Daube, B.C. ; WofSy, S.C.: Measurements of carbon sequestration by long-term eddy covariance: Methods and a critical evaluation of accuracy. In: Global Change Biology 2 (1996), S. 169-182 
[GML+96] Grace, J. ; Mahli, Y. ; Lloyd, J. ; MCIntyre, J. ; Miranda, A.C. ; Meir, P. ; Miranda, H.S.: The use of eddy covariance to infer the net carbon dioxide uptake of Brazilian rain forest. In: Global Change Biology 2 (1996), S. 209-217

[GMR $\left.{ }^{+} 04\right]$ Goulden, M.L. ; Miller, S.D. ; Rocha, H.R. da ; Menton, M.C. ; Freitas, H.C. de ; Silva Figueira, A.M. de ; Sousa, C.A.D. de: Diel and seasonal patterns of tropical forest $\mathrm{CO} 2$ exchange. In: Ecol. Appl. 14 (2004), S. 42-54

[Gra04] GRACE, J.: Understanding and managing the carbon cycle. In: Journal of Ecology 92 (2004), S. 189-202

[GRF03] Goeckede, M. ; Rebmann, C. ; Foken, T.: Use of footprint modelling for the characterisation of complex meteorological flux measurement sites. In: $E G S-A G U-E U G$ Joint Assembly, Abstracts from the meeting held in Nice, France (2003), April, S. 2004

[Gro87] Gross, G.: Some effects of deforestation on nocturnal drainage flow and local climate: A numerical study. In: Boundary-Layer Meteorology 38 (1987), März, S. 315-337

[Gro93] Gross, G.: Numerical Simulation of Canopy Flows. Springer Verlag, New York, 1993. - 168 S.

[HKB ${ }^{+}$94] Hollinger, D. Y. ; Kelliher, F. M. ; Byers, J. N. ; Hunt, J. E. ; McSeveny, T. M. ; Weir, P. L.: Carbon dioxide exchange between an undisturbed old-growth temperate forest and the atmosphere. In: Ecology 75 (1994)

[Hoj93] Hojstrup, J.: A statistical data screening procedure . In: Measurement Science and Technology 4 (1993), Februar, S. $153-157$

[HW92] Horst, T.W. ; WeIL, J.C.: Footprint estimation for scalar flux measurements in the atmospheric surface layer. In: Boundary-Layer Meteorol. 59 (1992), S. 279-296

[Ibr01] IBRom, A: Die biophysikalische Steuerung der Kohlenstoffbilanz in einem Fichtenbestand im Solling / Berichte des Forschungszentrums Waldökosysteme. 2001 (Reihe B). - Forschungsbericht. - 209 S.

$\left[\mathrm{IOJ}^{+} 07\right] \quad$ Kapitel Effects of land-use change on matter and energy exchange between ecosystems in the rain forest margin and the 
atmosphere. In: Ibrom, T. ; Oltchev, A. ; June, T. ; Ross, T. ; Kreilein, H. ; Falk, U. ; Merklein, J. ; Twele, G. A. ans R. A. ans Rakkibu ; Grote, G. ; Gravenhorst, G.: Stability of Tropical Rainforest Margins. Springer Verlag, Heidelberg, 2007, S. 289

[IRK $\left.{ }^{+}\right]$Ibrom, A. ; Ross, T. ; Kreilein, H. ; June, T. ; Oltchev, A. ; Falk, U. ; Rauf, A. ; Gravenhorst, G.: Large net CO2 uptake by a tropical upland rainforestin Central Sulawesi, Indonesia. In: Agric. For. Meteorol. (accepted)

[IST ${ }^{+}$96] Ibrom, A. ; Schuetz, C. ; Tworek, T. ; Morgenstern, K. ; Oltchev, A. ; Falk, M. ; Constantin, J. ; Gravenhorst, G.: Eddy correlation measurements of the fluxes of $\mathrm{CO} 2$ and $\mathrm{H} 2 \mathrm{O}$ above a spruce stand. In: Physics and Chemistry of the Earth 5/6 (1996), S. 409-414

[Jin06] Jing, R.: Soil Respiration Measurements in a Tropical Rain Forest Margin. Georg-August-Universität-Göttingen, 2006

[Jon92] Jones, H.G.: Plants and Microclimate. Cambridge University Press, 1992. - $428 \mathrm{~S}$.

[KA98] Katul, G.G. ; Albertson, J.D.: An Investigation of Higher-Order Closure Models for a Forested Canopy. In: Boundary-Layer Meteorol. 8 (1998), S. 47-74

[Kan04] Kantha, L. H.: The length scale equation in turbulence models. In: Nonlinear Processes in Geophysics 1 (2004), S. 83-97

[KF94] Kaimal, J.C. ; Finnigan, J.J.: Atmospheric Boundary Layer Flows: their Structure and Measurement. Oxford University Press, 1994. - 289 S.

[KFP ${ }^{+}$06] KATUL, G.G. ; FINNIGAN, J.J. ; POGGI, D. ; LEUNING, R. ; BELCHER, S.E.: The influence of hilly terrain on canopy-atmosphere carbon dioxide exchange. In: Boundary-Layer Meteorology 118 (2006), S. 189-216

[KM01] Kormann, R. ; MeIXner, F.X.: An analytical footprint model for non-neutral stratification. In: Boundary-Layer Meteorol. 99 (2001), S. 207-223

[KMOW97] Kristensen, L ; Mann, J. ; Oncley, S.P. ; WyngaArd, J.C.: How close is close enough when measuring scalar fluxes 
with displaced sensors. In: J Atmos Oceanic Tech 14 (1997), S. $814-821$

[KMPS04] Katul, G.G. ; Mahrt, L. ; Poggi, D. ; Sanz, C.: One and two equation models for canopy turbulence. In:

Boundary-Layer Meteorology 113 (2004), S. 81-109

[KS04] KlaAssen, W. ; Sogachev, A.: Flux Footprint Simulation in Complex Terrain. In: AGU Fall Meeting Abstracts (2004), Dezember, S. C5+

[LAM98] Lindroth, A. ; A., Grelle ; Moren, A.S.: Long-Term Measurements of Boreal Forest Carbon Balance Reveal Large Temperature Sensitivity. In: Global Change Biol. 4 (1998), S. $443-450$

[LBA99] Law, B.E. ; Baldocchi, D.D. ; Anthoni, P.M.: Below canopy and soil CO2 fluxes in a ponderosa pine forest. In: Agricultural and Forest Meteorology 94 (1999), S. 171-188

[LC03] Loescher, S.F. Oberbauer H.L. G. H.W. ; Clark., D.B.: Environmental controls on net ecosystem-level carbon exchange and productivity in a Central American tropical wet forest. In: Global Change Biology 9 (2003), S. 396-412

[Lee98] LEE, X.: On Micrometeorological Observation of Surface-Air Exchange over Tall Vegetation. In: Agric. For. Meteorol. 91 (1998), S. 39-50

[LG97] Landsberg, J.J. ; Gower, S.T.: Applications of Physiological Ecology to Forest Management. Academic Press, New York, 1997. - 354 S.

[LGM95] Lloyd, J. ; Grace, J. ; Miranda, A.C: A simple calibrated model of Amazon rain forest productivity based on leaf biochemical properties. In: Plant, Cell and Environment 18 (1995), S. 1129-1145

[LH02] LEE, X. ; Hu, X.: Forest-air fluxes of carbon, water, and energy over non-flat terrain. In: Boundary-Layer Meteorol. 103 (2002), S. 277-301

[Lic03] LI-COR: Open Path CO2/H2O Analyser, Instruction Manual. (2003) 
[LL04] LEE, W. X.and M. X.and Massman ; LAW, B.: Handbook of Micrometeorology: A Guide for Surface Flux Measurement and Analyses. 2004. - $245 \mathrm{~S}$.

[LL05] Kapitel Coordinate Systems and Flux Bias Error. In: LEE, W. X.and M. X.and Massman ; LAw, B.: Handbook of Micrometeorology: A Guide for Surface Flux Measurement and Analyses. 1th. Kluwer Academic Puplishers, Dordrecht, 2005.

- ISBN 1-4020-2264-6

[LPf01] Liu, H. ; Peters, G. ; Foken, T.: New Equations For Sonic Temperature Variance And Buoyancy Heat Flux With An Omnidirectional Sonic Anemometer. In: Boundary-Layer Meteorology 100 (2001), S. 459-468

[LRA +97$]$ Lavigne, M.B. ; Ryan, M.G. ; Anderson, D.E. ; BaldocChi, D.D. ; Crill, P.M. ; FitzJarrald, D.R. ; Goulden, M.L. ; Gower, S.T. ; MAssheder, J.M. ; McCaughey, J.H. ; Rayment, M. ; Striegl, R.G.: Comparing nocturnal eddy covariance measurements of ecosystem respiration made by scaling chamber measurements at six coniferous boreal sites. In: Jour. of Geophys. Research 102, No. D24 (1997), S. 77-85

$\left[\mathrm{MBH}^{+} 04\right]$ Morgenstern, K. ; Black, T.A. ; Humphreys, E.R. ; Griffis, T.J. ; Drewitt, G.B. ; CAi, T. ; Nesic, Z. ; Livingston, N.J.: Sensitivity and uncertainly of the carbon balance of a Pacific Northwest Douglas-fir forest during an El Nino/La Nina Cycle. In: Agricultural and Forest Meteorology 123 (2004), S. 201-219

[MBJ99] Malhi, Y. ; Baldocchi, D. D. ; Jarvis, P. G.: The carbon balance of tropical, temperate and boreal forests. In: Plant, Cell and Environment 22 (1999), S. 1-26

[McM88] McMillen, R.T.: An eddy correlation technique with extended applicability to nonsimple terrain. In: Bound. Layer Meteorol. 43 (1988), S. 231-245

[MCM $\left.{ }^{+} 05\right]$ Marcolla, B. ; Cescatti, A. ; Montagnani, L. ; Manca, L. ; Kerschbaumer, S. G.and M. G.and Minerbi: The Role of advective fluxes in the carbon balance of an alpine coniferous forest. In: Agricultural and Forest Meteorology 130 (2005), S. 193-206 
[MGM+04] Miller, S.D. ; Goulden, M.L. ; Menton, M.C. ; Rocha, H.R. da ; Freitas, H.C. de ; Figueira, A. ; Sousa, C. de: Biometric and micrometeorological measurements of tropical forest carbon balance. In: Ecological Applications 14 (2004), S. $114-126$

[ML02] Massman, W.J. ; LEE, X.: Eddy covariance flux corrections and uncertainties in long-term studies of carbon and energy exchanges. In: Agric. For. Meteorol. 113 (2002), S. 121-144

[Mml96] Moncrieff, J.B. ; Mahli, Y. ; Leuning, L.: The propagation of errors in long-term measurements of land-atmosphere fluxes of carbon and water. In: lobal Change Biology 2 (1996), S. 231-240

[MO58] Kapitel "Fundamentale Gesetzmäßigkeiten der turbulenten Vermischung in der bodennahen Schicht der Atmosphäre.". In: Monin, A.S. ; OBukhov, A.M.: Sammelband zur statistischen Turbulenz. Akademie-Verlag Berlin, 1958

[Mor00] Morgenstern, K.: Turbulent CO2-, H2O- and Energyfluxes above a Mediterranean Oak and a Mountainous Spruce Forest Investigated by Eddy-Covariance Measurements. Georg-August-Universität-Göttingen, Bioklimatologie, Diss., 2000. - $106 \mathrm{~S}$.

[MVN01] Mahrt, L. ; Vickers, D. ; Nakamura, R.: Shallow Drainage Flows. In: Boundary-Layer Meteorology 101 (2001), S. 243-260

[OCN ${ }^{+}$02] Oltchev, A. ; CCaronermak, J. ; Nadezhdina, N. ; TAtarinov, F. ; Tischenko, A. ; Ibrom, A. ; Gravenhorst, G.: In: Boreal Environ Res 7 (2002), 389-397 S.

[PBM00] PAW, K.T. ; Baldocchi, D.D. ; Meyers, T.P.: Correction nts incorporating both advective effects and density fluxes. In: Bound. Layer Meteorol. 97 (2000), S. 487-511

[PBMW00] Paw, K.T. ; Baldocchi, D.D. ; Meyers, T.P. ; Wilson, KB.: Correction of eddycovariance measurements incorporating both advective effects and density fluxes. In: Bound. Layer Meteorol. 97 (2000), S. 487-511

[PD88] Panofsky, H. A. ; Dutton: An Introduction to Boundary Layer Meteorology. Kluwer Academic Publishers, 1988. - 122 S. 
[Pie84] PiElke: Mesoscale meteorological modeling. Academic Press, New York, 1984. - 612 pp. S.

[Pop00] Pope, S.B.: Turbulent Flows. Cambridge University Press., 2000. - $687 \mathrm{~S}$.

[Pra25] PRANDTL, L: Bericht ueber Untersuchungen zur ausgebildeten Turbulenz. In: Z. f. angew. Math. Mech. 5 (1925), S. 136-139

[PRA ${ }^{+}$06] Papale, D. ; Reichsteinand, E. M. C. M. Canfora ; Aubinet, M. ; Bernhofer, C. ; Longdoz, B. ; Kutsch, W. ; Ramba, S. ; VAlentini, R. ; Vesala, T. ; YakiR, D.: Towards a more harmonized processing of eddy covariance CO2 fluxes:algorithms and uncertainty estimation. In: Biogeosciences Discuss. 3 (2006), S. 961-992

[PTlW77] Panofsky, H. A. ; Tennekes, H. ; Lenschow, D. H. ; WyngaARD, A.: Methods and limitations in validation of footprint models. In: Boundary-Layer Meteorol. 11 (1977), S. 355-361

[Pur05] Purbopuspito, J: Trace gas emmissions from forest and land-use systems at the tropical forest margins in Central Sulawesi, Indonesia. Georg-August-Universität-Göttingen, Bodenkunde, Diss., 2005. - 88 S.

[RAK00] Rannik, Ü. ; Aubinet, M. ; Kurbanmuradov, O.: Footprint Analysis For Measurements Over A Heterogeneous Forest. In: Boundary-Layer Meteorology 97 (2000), S. 137-166

[Reb03] Rebmann, C.: Kohlendioxid-, Wasserdampf- und Energieaustausch eines Fichtenwaldes in Mittelgebirgslage in Nordostbayern. Universität Bayreuth, Fakultät für Biologie, Chemie und Geowissenschaften, Diss., 2003. - 141 S.

[RGF $\left.{ }^{+} 05\right]$ Rebmann, C. ; Göckede, M. ; Foken, T. ; Aubinet, M. ; Aurela, M. ; Berbigier, P. ; Bernhofer, C. ; Buchmann, N. ; Carrara, A. ; Cescatti, A. ; Ceulemans, R. ; Clement, R. ; Elbers, J. A. ; Granier, A. ; Grünwald, T. ; Guyon, D. ; Havránková, K. ; Heinesch, B. ; Knohl, A. ; Laurila, T. ; Longdoz, B. ; Marcolla, B. ; Markkanen, T. ; Miglietta, F. ; MoncriefF, J. ; Montagnani, L. ; Moors, E. ; Nardino, M. ; Ourcival, J.-M. ; RAmbal, S. ; RAnNiK, Ü. ; RotenberG, E. ; Sedlak, P. ; Unterhuber, G. ; Vesala, T. ; Yakir, D.: 
Quality analysis applied on eddy covariance measurements at complex forest sites using footprint modelling. In: Theoretical and Applied Climatology 80 (2005), April, S. 121-141.

http://dx.doi.org/10.1007/s00704-004-0095-y. - DOI $10.1007 / \mathrm{s} 00704-004-0095-\mathrm{y}$

[RIS $\left.{ }^{+}\right]$Ross, T. ; Ibrom, A. ; Sogachev, A. ; Panferov, O. ; Gravenhorst, G.: Experimental and numerial methods to estimate advective fluxes in complex terrain. In:

Boundary-Layer Meteorology (in prep.)

[Ros03] Ross, T: Turbulente Grenzschichten: Ein Vergleich zwischen Atmosphaere und Rayleigh-Benard Experiment. Georg-August-Universität-Göttingen, 2003

[RS82] Raupach, M. R. ; Shaw, R. H.: Averaging Procedures for Flow within Vegetation Canopies. In: Boundary-Layer Meteorol. 61 (1982), S. 47-64

[RWCH92] Raupach, M.R. ; Weng, W.S. ; Carruthers, D.J. ; Hunt, C.J.: Temperature and humidity fields and fluxes over low hills. In: Q.J.R. Meteorol. Soc 118 (1992), S. 191-225

[San03] SAnz, C.: A Note on k- $\epsilon$ Modelling of Vegetation Canopy Air-Flows. In: Boundary-Layer Meteorol. 108 (2003), S. 191-197

[SB83] Schotanus, Nieuwstadt F. P. ; Bruin, H. de: Temperature measurement with a sonic anemometer and its application to heat and moisture fluxes. In: Boundary-Layer Meteorol. 26 (1983), S. 81-93

[Sch02] Schmid, H. P.: Footprint modeling for vegetation atmosphere exchange studies: A review and perspective. In: Agric. Forest Meteorol. 67 (2002), S. 159-183

[Sch05] SCHRÖTER, J.: Advective influence on the CO2-exchange of a forest site. Universität Bayreuth, 2005

[SHG86] SpitTers, C. ; H., Toussanint ; Goudriann, J.: Separating the diffuse and direct component of global radiation and its implications for modeling canopy photosynthesis. Part I. In: Agric. For. Meteorol. 38 (1986), S. 217-229 
[SIB02] Soler, M.R. ; Infante, C. ; Buenestado, P.: Observations Of Nocturnal Drainage Flow In A Shallow Gully. In: Boundary-Layer Meteorology 105 (2002), S. 253-273

[SK88] SATO, T. ; Kondo, J.: A simple model of drainage flow in a valley. In: Boundary-Layer Meteorology 45 (1988), Dezember, S. $355-369$

[SKBN04] Sodhi, N.S. ; KoH, L.P. ; Brook, B.W. ; NG, P.K.L.: Southeast Asian biodiversity: the impending disaster. In: Trends in Ecology and Evolution 19 (2004), S. 654-660

[SL04] SogACHEV, A. ; Lloyd, J.: Using a one-and-a-half order closure model of the atmospheric boundary layer for surface flux footprint estimation. In: Boundary-Layer Meteorol. 112(3) (2004), S. 467-502

[SLK ${ }^{+}$05] Sogachev, A. ; Leclerc, M.Y. ; Karipot, A. ; Zhang, G. ; VESALA, V.: Effect of clearcuts on footprints and flux measurements above a forest canopy. In: Agricultural and Forest Meteorology 133 (2005) 182-196 133 (2005), S. 182-196

[SLMD90] SchuepP, P.H. ; Leclerc, M.Y. ; MacPherson, J.I. ; DESJARDins, R.L.: Footprint prediction of scalar fluxes from analytical solutions of the diffusion equation. In:

Boundary-Layer Meteorology 50 (1990), S. 355-373

[SMhl02] Sogachev, A. ; Menzhulin, G.V. ; Heimann, M. ; Lloyd, J.: A simple three-dimensional canopy - planetary boundary layer simulation model for scalar concentrations and fluxes. In: Tellus, Series B - Chemical and Physical Meteorology 54 (2002), S. 784-819

[SMM+03] Saleska, S.R. ; Miller, S.D. ; Matross, D.M. ; Goulden, M.L. ; Wofsy, S.C. ; Rocha, H da ; Camargo, P.B. de ; Crill, P.M. ; Daube, B.C. ; Freitas, C. ; Hutyra, L. ; Keller, M. ; Kirchhoff, V. ; Menton, M. ; Munger, J.W. ; Pyle, E.H. ; Rice, A.H. ; Silva, H.: Carbon in Amazon forests: unexpected seasonal fluxes and disturbance-induced losses. In: Science 302 (2003), S. 1554-1557

[SMSD86] Sellers, P.J. ; Mintz, Y. ; Sud, Y.C. ; Dalcher, A.: A simple biosphere model ( $\mathrm{SiB})$ for use within general circulation models. In: J. Atmos. Sci. 43 (1986), S. 505-531 
[SP06] Sogachev, A. ; Panferov, O.: Modification of two-equation models to account for plant drag. In: Boundary-Layer Meteorol. 121 (2006), S. 229-266

[SPGV05] Sogachev, A. ; Panferov, O. ; Gravenhorst, G. ; VESALA, T.: Numerical analysis of flux footprints for different landscapes. In: Theor Appl Climato 80 (2005), S. 169-185

[SRV04] Sogachev, A. ; Rannik, Ü. ; Vesala, T.: Flux footprints over complex terrain covered by heterogeneous forest. In: Agric. For. Meteorol. 112 (2004), S. 143-158

[Stu84] Stull, R.B: Atmospheric Turbulence,Models and Methods for Engineering Applications. J.Wiley, New York, 1984. - 397 S.

[TAB $\left.{ }^{+} 03\right]$ Turnipseed, A. A. ; Anderson, D. E. ; Blanken, P. D. ; Baugh, W. M. ; Monson, R. K.: Airflows and turbulent flux measurement in mountainous terrain part1. canopy and local effects. In: Agric. For. Meteorol. 119 (2003), S. 1-21

[TT69] Tanner, C. B. ; Thurtell, G.W.: Anemoclinometer Measurements of Reynolds Stress and Heat Transport in the Atmospheric Surface Layer: Reasearch and Development Tech. Report / University of Wisconsin. 1969 (ECOM-66-G22-F). Forschungsbericht

[VM97] Vickers, D. ; MAhrT, L.: Quality control and flux sampling problems for tower and aircraft data. In: J. Atm. and Oc. Tech. 14 (1997), 512-526. citeseer.ist.psu.edu/vickers97quality.html

[VMD $\left.{ }^{+} 00\right]$ Valentini, R. ; Matteucci, C. ; Dolman, A.J. ; Schulze, E.D. ; Rebmann, C. ; Moors, E.J. ; Granier, A. ; Gross, P. ; Jensen, N.O. ; Pilegaard, K. ; Lindroth, A. ; Grelle, A. ; Bernhofer, C. ; Grünwald, T. ; Aubinet, M. ; Ceulemans, R. ; Kowalski, A.S. ; Vesala, T. ; Rannik, Ü ; Berbigier, P. ; Loustau, D. ; Gudmundsson, J. ; Thorgeirsson, H. ; Ibrom, A. ; Morgenstern, K. ; Clement, R. ; Moncrieff, J. ; Montagnani, L. ; Minerbi, S. ; JaRVis, P.G.: Respiration as the main determinant of carbon balance in European forests. In: Nature 404 (2000), S. 861-86

[WDC $\left.{ }^{+} 05\right]$ Wang, W. ; Davis, K.J. ; CoOK, B.D. ; Bakwin, P.S. ; Yi, C. ; Butler, M.P. ; Ricciuto, D.M.: Surface layer CO2 
budget and advective contributions to measurements of net ecosystem-atmosphere exchange of CO2. In: Agric. For. Meteorol. 135 (2005), S. 202-214

[WFR98] Wilson, J. D. ; Finnigan, J. J. ; Raupach, M. R.: Plant-Canopy Flows, and its Application to Winds in a Canopy on a Ridge. In: Quart. J. Roy. Meteorol. Soc. 124 (1998), S. 705-732

[WMn98] Williams, M. ; Malhi, Y. ; Nobre, A.D: Seasonal variation in net carbon dioxide exchange and evapotranspiration in a Brazilian rain forest. In: Plant, Cell and Environment 21 (1998), S. 953-968

[WOS01] Wilczak, J. ; Oncley, S. ; Stage, S.: Sonic anemmometer tilt correction algorithms. In: Boundary-Layer Meteorol. 260 (2001), S. 127-150

[WP80] WebB, E.K. ; Pearman, R. G.I.and L. G.I.and Leuning: Correction of the flux measurements for density effects due to heat and water vapour transfer. In: Quarterly Journal of The Royal Meteorological Society, 106106 (1980), S. 85-100

[WWR78] Woodwell, G.M. ; Whittaker, R.H. ; Reiners, W.A.: The biota and the world carbon budget. In: Science 199 (1978), S. 141-146

[WY03] Wilson, J.D. ; YeE, E.: Calculation of Winds Disturbed by an Array of Fences. In: Agric. For. Meteorol. 115 (2003), S. 31-50

[YM06] YAng, Raupach M.R. Shaw R.H. Paw U K. B. ; Morse, A.P.: Large-eddy Simulation of Turbulent Flow across a Forest Edge. Part I: Flow Statistics. In: Boundary-Layer Meteorology 120 (2006), S. 377-412 


\section{Abbildungsverzeichnis}

2.1 Kartesisches Kontrollvolumen über einem Waldbestand (Quelle (nicht publ.): C. Feigenwinter). . . . . . . . . . . 7

2.2 Schematische Darstellung einer konvergenten (links) bzw. divergenten Strömung (rechts) durch ein Kontrollvolumen. Außerdem dargestellt (zur Illustration von $\langle\bar{C}\rangle$ ): Ein vertikales $\mathrm{CO}_{2}$-Konzentrationsprofil, wie es typischerweise in der Nacht

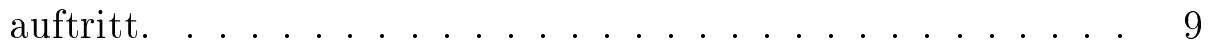

2.3 Das Untersuchungsgebiet auf der Insel Sulawesi/Indonesien östlich der Wallace Line. Quelle: [Die07] (modifiziert) . . . . . 11

2.4 Geländerelief am Turmstandort Bariri. Die Position des Messturmes ist in der Mitte der Abbildung gekennzeichnet. (Entsprechend der Konvention weist Nord in Richtung oberer Bildrand.) . . . . . . . . . . . . . . . . . 12

2.5 (a) Vegetationskarte im UTM-Koordinatensystem für das Messgebiet. (b) Darstellung Blattflächendichte (Leaf Area Density) für das geschlossene Waldgebiet um den Turmstandort herum. . . . . . . . . . . . . . . . . . . . 12

2.6 Bild (a) zeigt die $70 \mathrm{~m}$ hohe Turmkonstruktion. Kurz oberhalb des Waldbestandes befindet sich die Fotovoltaikanlage zur Stromversorgung (oberer Bildrand). Das Eddy-Kovarianz Messsystem (b) setzt sich aus aus einem Ultraschallanemometer USA-1 der Firma METEK und einem "Open-Path"Infrarot-Gasanalysator (IRGA) der Firma LICOR zusammen. 
2.7 Schematische Darstellung (a) des Messturms am Standort Bariri. Folgende Messgrößen wurden aufgezeichnet: $U$, die Windgeschwindigkeit respektive -richtung, $T_{a}$, die Lufttemperatur, $H_{r}$, die rel. Feuchte, $R_{k}$ und $R_{l}$, die kurz- und die langwellige Strahlung. Das EK-Messystem für den kontinuierlichen Messbetrieb befindet sich $48 m$ über Grund an einem (b) $4.5 \mathrm{~m}$ langen Ausleger, welcher nach Richtung SSO zeigt. Mit dem nicht-stationären Messsystem wurden nacheinander in $56 m$ Höhe und in $48 m$ Höhe (vis-a-vis des stationären Systems) Vergleichsmessungen durchgeführt (nicht dargestellt). . 14

2.8 Bestimmung des Koordinatensystems nach dem Planar-Fit Verfahren (Illustration). $u, v$ und $w$ bezeichnen die mittleren Windkomponenten, durch welche die Referenzebene festgelegt wurde, Ref.: [WOS01]. . . . . . . . . . . . . .

2.9 Darstellung der Schubspannungsgeschwindigkeit $u_{*}$ bzw. des $\mathrm{CO}_{2}$-Flusses zweier aufeinanderfolgender Tage (11.02 bis 13.02 . 2004) zur Illustration des Zusammenhanges zwischen Turbulenzgrad und hohen bzw. niedrigen Flusswerten bei Nacht (vereinzelt Datenlücken). Der schattierte Bereich stellt ein gleitendes 3-Stundenmittel dar. Infolge schwach ausgeprägter Turbulenz (niedrige Schubspannungsgeschwindigkeiten bei stabiler atmosphärischer Schichtung) lässt sich der $\mathrm{CO}_{2}$-Fluss in diesen Zeiten nicht adäquat mit der EK-Methode alleine bestimmen. . . . . . . . . . . . . . 28

2.10 Zur Bestimmung des Wolkenbedeckungsgrades aus $\left(R_{G} / R_{0}\right)$. Die Grafik zeigt beispielhaft zwei Tage unterschiedlicher Wolkenbedeckung. Während am 30.07.04 klare Wetterverhältisse vorherrschten, weist der 19.08.04 mehr bedeckte Verhältnisse auf (vergl. Tab. 2.2). Die Quadrate bezeichnen $R_{0}$, die Kreise

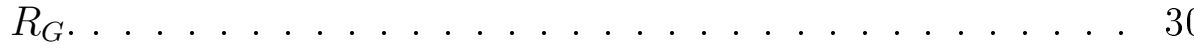

2.11 Bestimmung des Quellgebiets der Hauptwindrichtungen nach dem analytischen Modell von Kormann-Meixner. Die Ellipsenparameter $a, b$ und $c$ entsprechen dem Mittelwert des auf Halbstundenbasis berechneten Quellgebiets aus dem 90\% des Flusses stammt. Ref::[KM01].

3.1 Windrose des Jahres 2004. Dargestellt ist die prozentuale Häufigkeit der einzelnen Windklassen. Die Messungen aus 48m Höhe wurden mit dem Ultraschallanemometer (USA-1, METEK) durchgeführt. . . . . . . . . . . . . . . 46 
3.2 Dargestellt ist der Tagesgang (a) der mittleren Windrichtung für alle Windgeschwindigkeiten respektive (b) der Tagesgang der gemittelten Horizontal- $(U)$ und Vertikalwindgeschwindigkeit $(w)$ sowie des Stabilitätsparameters $(\zeta)$ und der Schubspannungsgeschwindigkeit $\left(u_{*}\right)$ des Jahres 2004. . . . . . . . . 47

3.3 Der saisonale Gang der relevantesten meteorologischen Variablen für die Messperiode (10/2003 - 06/2005). Dargestellt sind Globalstrahlung $\left(R_{G}\right)$, Lufttemperatur $(T)$, Wasserdampfdefizit $(V P D)$ und der Niederschlag $\left(N_{d}\right)$ aus $48 m$ Höhe im Tagesmittel. Zusätzlich wurde (rot) ein gleitendes 10-Tagesmittel auf die Daten angewandt. . . . . . . . . . . . .

3.4 Kumulativ dargestellter Niederschlag für die gesamte Messperiode. Deutlich zu erkennen: die regnerische $(6.6 \mathrm{~mm} / \mathrm{Tag})$ und weniger regnerische $(3.9 \mathrm{~mm} / \mathrm{Tag})$ Jahreshälfte 2004 . . . . . 50

3.5 Bowen Ratio (Verhältnis von sensiblem zu latentem Wärmestrom): Dargestellt ist das Tagesmittel für $R_{G}>50 \mathrm{Wm}^{-2}$. Zusätzlich wurde (rot) ein gleitendes 10-Tagesmittel auf die Daten angewandt.

3.6 Dargestellt ist (a) die Funktion $f_{\text {korr }}$ zur Korrektur der turmbedingten Dämpfung der Vertikalwindfluktuationen $\left(\sigma_{w}\right)$ und (b) die relative Häufigkeit der Vertikalwindfluktuationen in Abhängigkeit von der Windrichtung für das stationäre Ultra-

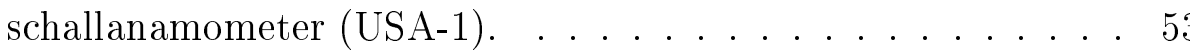

3.7 Quellgebietsanalyse für den Nettofluss über flachem und über komplexem Gelände. Der Turmstandort befindet sich im Koordinatenursprung $(0,0)$. Die Abbidungen a, c und e stellen die Quellgebietsbereiche über homogener und die Abbildungen b,d und $\mathrm{f}$ über realer $\left(z=48 \mathrm{~m}, z_{0}=2.7 \mathrm{~m}\right.$ ) Orographie (farbige Bereiche) dar. Dargestellt sind die 50, 70 und 90\% Isoplethe (siehe Bezeichnungen auf den Abbildungen) der Quellgebietsfunktion. Die vorgegebene Geostrophische Windgeschwindigkeit beträgt $G=10 \mathrm{~m} \mathrm{~s}^{-1} \ldots \ldots \ldots \ldots$. . . . . . . 5

3.8 Quellgebietsanalyse mit dem analytischen Footprint-Modell nach [KM01]. Die dargestellten Quellgebiete (Versatzhöhe: $d=27.3 \mathrm{~m}$, Rauhigkeitslänge: $z_{0}=2.7 \mathrm{~m}$ ) berücksichtigen $90 \%$ des gemessenen Flusses der Hauptwindrichtungen im Luv des Messturmes für jeweils stabile und neutrale Schichtung. . . . 56 
3.9 Überprüfung der Schicht höhenkonstanter Flüsse: Der $\mathrm{CO}_{2^{-}}$ Fluss $\left(F_{C}\right)$, die Schubspannungsgeschwindigkeit $\left(u_{*}\right)$ (als Quadratwurzel des Impulsflusses $\left.\sqrt{\mid \overline{u^{\prime} v^{\prime} \mid}}\right)$ und der sensible $(H)$ respektive der latente Wärmefluss $(\lambda E)$ aus zwei Höhen $(z-d=$ $20.7 \mathrm{~m}$ respektive $28.7 \mathrm{~m}$ ) ist jeweils gegeneinander dargestellt.

3.10 Qualitätstest der integralen Turbulenzcharakteristik nach dem Modell von Panofsky et al. [PD88]. Dargestellt ist die normierte Standardabweichung der Vertikalwindkomponente für (a) $48 \mathrm{~m}$ und (b) $56 \mathrm{~m}$ über dem Bodenrelief. Die unterbrochenen Linien geben $\pm 30 \%$ Schranken an, wie sie von Foken und Wichura [FW96] zur Markierung des Toleranzbereichs vorgeschlagen wurden. . . . . . . . . . . . . .

3.11 Relative Häufigkeit von instationären $\mathrm{CO}_{2}$-Halbstundenzeitreihen und Häufigkeitsverteilung aller $\mathrm{CO}_{2}$-Flüsse insgesamt (siehe Abb. in der Abb.) in Abhängigkeit des Stabilitätsparameters $\zeta$. 61

3.12 (a) Normalisierte $\mathrm{CO}_{2}$-Nachtflüsse $\left(F_{C} / F_{C \max }\right)$ gemittelt über sich überlappende Unterdatensätze der Fensterbreite $0.2 \mathrm{~m} / \mathrm{s}$ und (b) die relative Häufigkeitsverteilung der verfügbaren Daten in Abhängigkeit von $u_{*} . \ldots \ldots$. . . . . . . . . . .

3.13 Tagesmittel der turbulent transportierten Energie als Funktion der verfügbaren Energie für das Jahr 2004. Lediglich Tage, an denen mehr als 24 Halbstundenstatistiken zu Verfügung standen, wurden einbezogen. (Regression: rot, unterbrochene Linie, 1:1-Linie: schwarz) . . . . . . . . . . . . . .

3.14 (a) Verlauf der modellierten Strömungslinien am Beispiel eines vertikalen Schnitts durch Strömungsregime und Bodenrelief in WO -Richtung. Der Pfeil markiert die Windrichtung und $X=0$ markiert den Turmstandort. (b) Darstellung der Reliefoberfläche im Bereich der Messungen in NS und WO Richtung zur Überprüfung der Planar-Fit Winkel $(X, Y=0$ bezeichnet den Turmstandort). . . . . . . . . . . . 66

3.15 (a) SVAT-modellierte Konzentrationsprofile am Beispiel des 01.11 .2004 im Tagesverlauf und (b) Vergleich des modellierten mit dem gemessenen (Ein-Punkt-Messung) mittleren Speicherfluss im Tagesgang. Verwendet wurden alle Daten des Jah-

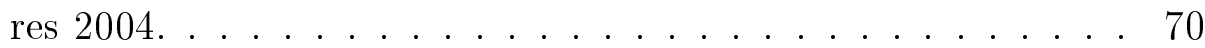


3.16 (a) Normalisierter Speicherterm (wobei der schattierte Bereich den Unsicherheitbereich, wie er durch eine 50\%-ige Überschätzung der Bodenrespiration verursacht würde, darstellt) und (b) vertikaler $\mathrm{CO}_{2}$-Fluss $\left(F_{a d v}\right)$ sowie mittlere Vertikalwindgeschwindigkeit $(w)$ in Abängigkeit von der Schubspannungsgeschwindigkeit $\left(u_{*}\right)$ aus allen verfügbaren Nachtdaten des Untersuchungszeitraumes (Messhöhe: $48 m$ ) . . . . . . . . . 72

3.17 Mittlerer Tagesgang (a) der $\mathrm{CO}_{2}$-Konzentration und (b) des $\mathrm{CO}_{2}$-Gesamtflusses $F_{C}+F_{S}$ sowie des Speicherflusses $F_{S}$ separat aus allen verfügbaren Daten.

3.18 Abhängigkeit der mittleren Vertikalwindgeschwindigkeit $w$ und ihrer Varianz $\sigma_{w}$ von dem Stabilitätparameter $\varsigma$. . . . . 76

3.19 Normalisierte $(\mathrm{a}, \mathrm{b})$ assimilatorische und $(\mathrm{c}, \mathrm{d})$ respiratorische $\mathrm{CO}_{2}$-Flüsse $\mathrm{FCO}_{2}\left(x_{1,2}, 48\right) / \mathrm{FCO}_{2}(-\infty, 48)$ im vertikalen Schnitt durch das $3 d$-Strömungsregime bei fest vorgegebenem geostrophischen Wind aus $300^{\circ}$ und neutraler atmosphärischer Schichtung. In $x$-Richtung verläuft der Schnitt von $\mathrm{W}$ nach $\mathrm{O}$ und in $y$-Richtung von $\mathrm{N}$ nach $\mathrm{S} ;\left(x_{1,2}=0\right)$ bezeichnet den Turmstandort und die Messhöhe beträgt $z_{r}=48 \mathrm{~m}$. Als Vorgabe wurde aus den Messungen für die Bodenrespiration ein Wert von $5 \mu \mathrm{mol} \mathrm{m} \mathrm{m}^{-2} \mathrm{~s}^{-1}$ übernommen. Die $\mathrm{CO}_{2}$-Senkenstärke der Vegetation verläuft proportional zur vertikalen $L A D$ Verteilung. . . . . . . . . . . . . . . . . . . . . 78

3.20 (Wie Abb. 3.19 a-d) Dargestellt ist der normalisierte vertikale Netto- $\mathrm{CO}_{2}$-Fluss in einem dem Relief folgenden Koordinatensystem.

3.21 Modellierte vertikale $\mathrm{CO}_{2}$-Flüsse (neutrale atmosphärische Schichtung) und Strömungslinien über dem Untersuchungsgebiet. Dargestellt sind die normalisierten Vertikalflüsse (a,c,e) als auch die Strömungslinien (b,d,f) $48 m$ über dem Relief (für die Abb. b,d und $f$ ist das Relief farbig unterlegt) im horizontalen Schnitt. Die vorgegebenen Windrichtungen (Pfeil) entsprechen den Hauptwindrichtungen und betragen in meteorologischer zählweise $90^{\circ}(\mathrm{a}, \mathrm{b}), 240^{\circ}$ (c,d) und $300^{\circ}$ (e,f). $(\mathrm{X}, \mathrm{Y})=(0,0)$ gibt den Turmstandort an. . . . . . . . . . . 81 
3.22 Wie Abb. 3.19, jedoch werden lediglich die normalisierten Flüsse in Messhöhe (48m) dargestellt. Zusätzlich zum normalisierten $\mathrm{CO}_{2}$-Nettofluss sind zur Veranschaulichung die normalisierten respiratorischen und assimilatorischen Flüsse angegeben. Die modellierten Windrichtungen entsprechen den gemessenen Hauptwindrichtungen: $90^{\circ}(\mathrm{a}, \mathrm{b}), 240^{\circ}(\mathrm{c}, \mathrm{d}) .(\mathrm{X}, \mathrm{Y})=(0,0)$ gibt den Turmstandort an. Die Pfeile geben die $x$ - (WO) respektive die $y$ - Komponente (NS) der betrachteten Hauptwindrichtung an. . . . . . . . . . . . . .

3.23 Wie Abb. 3.22 a-d. Dargestellt ist die geostrophische Wind-

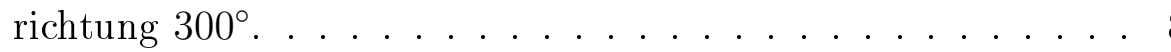

3.24 Erweiterung der Untersuchungen aus Abb. 3.21 (a,c,e) auf den gesamten Windrichtungsbereich. Angegeben sind die normalisierten Flüsse am Ort der EK-Messungen $48 m$ über Grund. . .

3.25 Darstellung des $\mathrm{CO}_{2}$-Flusses $\left(F_{C}+F_{S}\right)$ in Abhängigkeit von der fotosynthetisch aktiven Strahlung PPFD (photosynthetic photon flux density). Der Kurvenverlauf lässt sich durch die Michaelis-Menten Gleichung beschreiben. Nach Trennung des Datensatzes in Temperaturklassen von je $2^{\circ} \mathrm{C}$, wurde jede Temperaturklasse nochmals in drei Unterklassen nach Bewölkungsgrad (wolkenlos, bewölkt, bedeckt) unterteilt. Die Abbildungen zeigen beispielhaft die Temperaturklassen $20-22^{\circ} \mathrm{C}$ (a) und $22-24^{\circ} \mathrm{C}(\mathrm{b}) . \ldots \ldots \ldots \ldots \ldots$

3.26 Temperatur- und Strahlungsantwort des NEE unter bedeckten (a), bewölkten (b) und klaren (c) Himmelsbedingungen. (Einheit: $\mu \mathrm{mol} \mathrm{m}^{-2} \mathrm{~s}^{-1}$ ) . . . . . . . . . . . . . . 89

3.27 Gapfilling Prozedur: Fehlstellen $\leq 1.5 h$ wurden linear interpoliert. Grössere Lücken wurden nach Temperatur und Bewölkungsgrad mittels der Lichtsättigungskurven geschlossen. . . . 90

3.28 Saisonale und tageszeitliche Variation des $N E E\left(F_{C}+F_{S}\right)$. Alle Flüsse für $R_{G}>10 \mathrm{~W} / \mathrm{m}^{2}$ sind mit modellierten und interpolierten Werten geschlossen worden. Die nächtlichen Daten sind anschließend nach Anwenden des $u_{*}$-Filterkriteriums durch Zweimonatsmittel ersetzt worden. Der Zeitraum Sep./Okt. 2004 (siehe Pfeil) lässt etwas geringere $\mathrm{CO}_{2}$-Aufnahmeraten erkennen. . . . . . . . . . . . . . . . 91

3.29 Mittlerer Tagesgang der Lufttemperatur am Standort Bariri für das Jahr 2004. . . . . . . . . . . . . . . . . . . . 93 
3.30 Saisonaler Gang der Ökosystemrespiration: Die Respiration am Tage $R_{\text {Tag }}$ wurde nach der in $\left[\mathrm{IRK}^{+}\right]$beschriebenen Methode durch linearere Regression aus dem $\mathrm{CO}_{2}$-Fluss in Abhängigkeit von $R_{G}\left(<300 \mathrm{~W} \mathrm{~m}^{-2}\right)$ ermittelt. $R_{\text {Nacht }}$, die sog. dunkle Respiration, geht aus Mittlung der $u_{*}$ gefilterten Nachtwerte hervor. Für beide Methoden betrug die Mittelungslänge zwei Monate. Außerdem ist die Bodenrespiration, welche durch Bodenkammermessungen bestimmt wurde, mit Fehlertoleranz (gestrichene Linie) angegeben [Jin06]. . . . . . . . . . 94

3.31 (a) Kumulativ dargestellter $N E E\left(F_{C}+F_{S}\right)$, berechnet nach dem Planar-Fit und dem 2-d Rotation Verfahren. Die unterbrochene Linie stellt eine Steigung von $-2.81 g(C) / d$ dar. (b) Kumulativ dargestellter NEE (schwarz) als Summe seiner Komponenten $R E$ und GPR. . . . . . . . . . . . . . . . 96

3.32 Vergleich des turbulenten (EK) $\mathrm{CO}_{2}$-Flusses (in $\mu \mathrm{mol} \mathrm{m} \mathrm{m}^{-2} \mathrm{~s}^{-1}$ ), zum einen berechnet im Planar-Fit Koordinatensystem [WOS01] und zum anderen berechnet im 2- $d$ Rotationssystem [McM88]. Neben der roten Regressionslinie ist schwarz die 1 : 1 Linie dargestellt. . . . . . . . . . . . . . . . . 97 


\section{Tabellenverzeichnis}

2.1 Tabelle der durchgeführten Kalibrierungen der beiden IRGA's (LICOR-7500), Ref.:[Lic03]. Das stationäre Gerät ist als Nr. 1 und das mobile als Nr. 2 bezeichnet. (Nähere Erläuterungen: siehe Text.) . . . . . . . . . . . . . . 17

2.2 Beziehungen zwischen dem Verhältnis $\left(R_{d f, T} / R_{G, T}\right)$ und der atmosphärischer Durchlässigkeit $\left(R_{G, T} / R_{p o t, T}\right)$. Der tiefgestellte Index $T$ bezeichnet Tageswerte. Ref.: [SHG86] . . . . . . . 29

2.3 Zusammenstellung der verwendeten Modellparameter. . . . . . 36

2.4 Parameter zur Charakterisierung des Rauhigkeitseigenschaften des Regenwaldbestandes am Standort Bariri. Ref.: [IRK $\left.{ }^{+}\right]$

3.1 Ellipsenparameter (siehe Abb. 2.11) aus dem KM-Modell zur Darstellung der einzelnen Quellgebietsbereiche (vergl. Abb. 3.8). Es wurden entsprechend der drei Hauptwindrichtungen zwei atmosphärischen Stabilitäten (neutral/stabil) berechnet.

3.2 Resultate aus den Qualitätstests für die gesamte Messperiode. 60

3.3 Planar-Fit Ebenen Winkel $\alpha$ (Neigungungswinkel in WO Richtung) und $\beta$ (Neigungswinkel in NS Richtung) in Abhängigkeit von der Windrichtung und von der Windgeschwindigkeit.

3.4 Klassifikation der verschiedenen Advektionsmuster in Beziehung zur Quellstärkenverteilung und Massenflusscharakteristik Ref.: $\left[\mathrm{ABC}^{+} 05\right]$. HA: horizontale Advektion, VA: vertikale Advektion . . . . . . . . . . . . . . 75 
3.5 Regressionsparameter der Lichtsättigungsfunktion nach Michaelis Menten. Aufgrund der vernachlässigbar kleinen saisonalen Schwankungen der $\mathrm{CO}_{2}$-Aufnahmeraten konnte die gesamte Messperiode berücksichtigt werden. Dargestellt sind Temperatur $T\left(^{\circ}\right)$, Bewölkungsgrad, maximale $\mathrm{CO}_{2}$-Aufnahmerate $\mathrm{F}_{\mathrm{CO}_{2} \max }\left(\mu \mathrm{mol} \mathrm{m} \mathrm{s}^{-2}\right)$, Quantenausbeute $\alpha\left(\operatorname{mol}\left(\mathrm{CO}_{2}\right)\right.$ $m^{-1}$ absorb. Photonen), Tagatmung $R_{\text {day }}\left(\mu \mathrm{mol} \mathrm{m} \mathrm{m}^{-2} \mathrm{~s}^{-1}\right)$, Lichtsättigung $F_{\text {Sat }}\left(\mu \mathrm{mol} \mathrm{m} \mathrm{m}^{-2} \mathrm{~s}^{-1}\right)$ und das Bestimmtheitsmass $\left(R^{2}\right) \ldots \ldots \ldots \ldots$

3.6 NEE, berechnet aus den EK-Messungen nach der PlanarFit als auch nach der 2- $d$ Rotations-Methode. Angegeben ist der kumulierte $\mathrm{CO}_{2}$-Fluss über den gesamten Zeitraum der Messperiode (15.10.03-07.06.05) als auch die mittlere jährliche Summe in $g(C) m^{-2} \ldots \ldots \ldots$. . . . . . . . . 97 


\section{Lebenslauf}

\section{Zur Person:}

geb. am 08.06.1972

Geburtsort: Bad Oldesloe

Familienstand: ledig

Promotion

$06 / 2007$

Georg-August-Universität Göttingen

Abschluss der Promotion mit dem Thema: Eddy-Kovarianz

Messungen über einem tropischen Regenwald in komplexem

Gelände.

10/2004-heute Georg-August-Universität Göttingen

Fortsetzung der Promotion als Doktorand des Teilprojekts

(B1) des SFB 552 am Institut für Bioklimatologie in Göttingen: Auswertung der Messungen. Modellierung der atmos -phärischen Strömungsverhältnisse im Untersuchungsgebiet. Bewertung des Teilprojekts durch die DFG: exzellent

04/2004-10/2004 Tadulako University, Palu, Indonesia

Durchführen von meteorologischen und Eddy-Kovarianz

Messungen mittels Gerüstturm 70m über dem tropischen Regenwald.

Aufbau einer autarken Stromversorgung (Fotovoltaik). Anleitung eines 4-Mann Teams indonesischer Studenten. Durchführen von Sozialisationsverantstaltungen, um die lokale Landbevölkerung einzubeziehen.

Durchführen von Infrastrukturmaßnahmen zur Gewähr-

Studium: leistung des Forschungsbetriebs.

$11 / 2003$

Diplom-Physiker

$10 / 1998-11 / 2003$

Georg-August-Universität Göttingen

Studium der Physik, Schwerpunkte: Schwingungs- und Strömungsphysik. Diplomarbeit: Turbulente Grenzschichten: Ein Vergleich zwischen Atmosphäre und RayleighBènard Experiment.

09/1996 - 08/1997 Hochschule für angewandte Wissenschaften, Hamburg Studium der Elektrotechnik, Schwerpunkte: Netzwerke, Messtechnik, Programmieren. 
Berufspraxis:

02/2004-07/2006 Georg-August-Universität Göttingen

wissenschaftliche Hilfskraft. Diverse Tätigkeiten am

Institut für Bioklimatologie: Betreuung des meteorologischen Praktikums für Geowissenschaftler sowie Übungen der Physik für Forst- und Geowissenschaftler.

04/2003 - 02/2004 Georg-August-Universität Göttingen studentische Hilfskraft: Mitbetreuung des geophysikalisch-

Schule: en Praktikums.

03/1998 - 05/1998 Georg-August-Universität Göttingen

Fachbezogene Hochschulzugangsprüfung für den Studiengang Physik.

Göttingen, 24.05.2007

Thomas Roß 